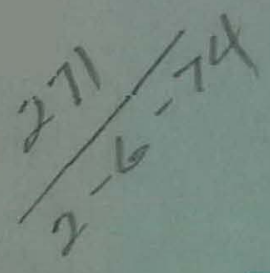

\title{
TEMPERATURE AND VELOCITY MEASUREMENTS AND PREDICTIVE MODEL COMPARISONS \\ IN THE NEAR-FIELD REGION \\ OF SURFACE THERMAL DISCHARGES
}

\section{by}

R. A. Paddock, A. J. Policastro,

\author{
A. A. Frigo, D. E. Frye,
}

and J. V. Tokar

\section{APPLIED TECHNOLOGY}

Any further distribution by any holder of this document or of the data therein to third parties representing foreign interests, foreign governments, foreign companies and foreign subsidiaries or foreign divisions of U.S. companies should be coordinated with the Director, Division of Reactor Research and Development, U.S. Atomic Energy Commission.-

\section{ANL \\ CES}

ARGONNE NATIONAL LABORATORY

\section{CENTER FOR ENVIRONMENTAL STUDIES}

Prepared for the U.S. ATOMIC ENERGY COMMISSION Division of Reactor Research and Development 


\section{DISCLAIMER}

This report was prepared as an account of work sponsored by an agency of the United States Government. Neither the United States Government nor any agency Thereof, nor any of their employees, makes any warranty, express or implied, or assumes any legal liability or responsibility for the accuracy, completeness, or usefulness of any information, apparatus, product, or process disclosed, or represents that its use would not infringe privately owned rights. Reference herein to any specific commercial product, process, or service by trade name, trademark, manufacturer, or otherwise does not necessarily constitute or imply its endorsement, recommendation, or favoring by the United States Government or any agency thereof. The views and opinions of authors expressed herein do not necessarily state or reflect those of the United States Government or any agency thereof. 


\section{DISCLAIMER}

Portions of this document may be illegible in electronic image products. Images are produced from the best available original document. 
The facilities of Argonne National Laboratory are owned by the United States Government. Under the terms of a contract (W-31-109-Eng-38) between the U. S. Atomic Energy Commission, Argonne Universities Association and The University of Chicago, the University employs the staff and operates the Laboratory in accordance with policies and programs formulated, approved and reviewed by the Association.

\section{MEMBERS OF ARGONNE UNIVERSITIES ASSOCIATION}

The University of Arizona

Carnegie-Mellon University

Case Western Reserve University

The University of Chicago

University of Cincinnati

Illinois Institute of Technology

University of Illinois

Indiana University

Iowa State University

The University of Iowa
Kansas State University The University of Kansas Loyola University Marquette University Michigan State University The University of Michigan University of Minnesota. University of Missouri Northwestern University University of Notre Dame
The Ohio State University

Ohio University

The Pennsylvania State University

Purdue University

Saint Louis University

Southern Illinois University

The University of Texas at Austin

Washington University

Wayne State University

The University of Wisconsin

\section{NOTICE}

This report was prepared as an account of work sponsored by the United States Government. Neither the United States nor the United States Atomic Energy Commission, nor any of their employees, nor any of their contractors, subcontractors, or their employees, makes any warranty, express or implied, or assumes any legal liability or responsibility for the accuracy, completeness or usefulness of any information, apparatus, product or process disclosed, or represents that its use would not infringe privately-owned rights.

Printed in the United States of America

Available from

U.S. Atomic Energy Commission

Technical Information Center

P.O. Box 62

Oak Ridge, Tennessee 37830

Price: Printed Copy $\$ 7.60$ 
AN亡/ES-25

Heat Rejection and

Utilization (UC -12)

\section{ARGONNE NATIONAL LABORATORY \\ 9700 South Cass Avenue \\ Argonne, Illinois 60439}

\section{TEMPERATURE AND VELOCITY MEASUREMENTS \\ AND PREDICTIVE MODEL COMPARISONS \\ IN THE NEAR-FIELD REGION \\ OF SURFACE THERMAL DISCHARGES}

by

R. A. Paddock, A. J. Policastro,

A. A. Frigo, D. E. Frye, and J. V. Tokar

Center for Environmental Studies

October 1973

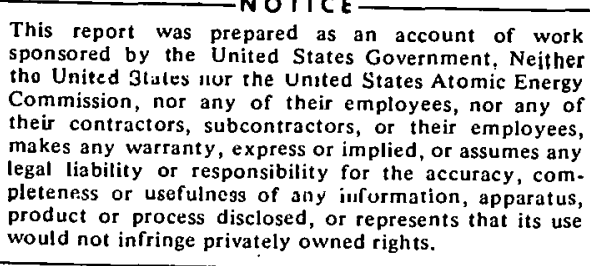

would not infringe privately owned rights. 
THIS PAGE

\section{WAS INTENTIONALLY LEFT BLANK}


TABLE OF CONTENTS

Page

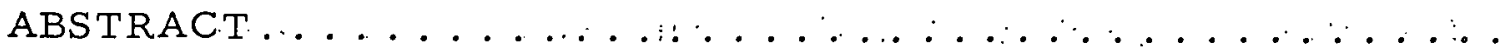

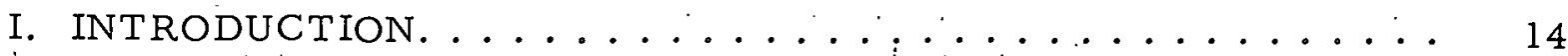

II. EXPERIMENTAL TECHNIQUE .......................... 16

III. DESCRIPTIONS OF POWER PLANTS. . . . . . . . . . . . 19

A. Point Beach Nuclear Power Plant. ............. 19

B. Palisades Nuclear Power Plant ............... 19

IV. RESULTS OF FIELD MEASUREMENTS . . . . . . . . . . . . 22

V. EXTRACTION OF JET CHARACTERISTICS FROM FIELD

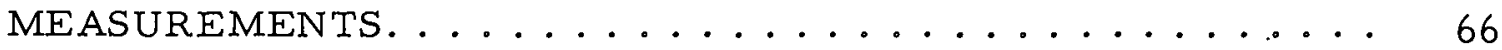

VI. MATHEMATICAL MODELING OF NEAR - FIELD REGION OF SURFACE THERMAL DISCHARGES . . . . . . . . . . 73

A. Motz-Benedict Model . . . . . . . . . . . . . 74

B. Stolzenbach-Harleman Model. ............. 78

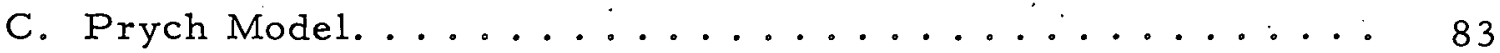

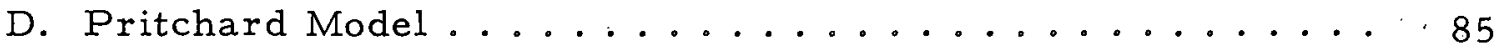

VII. MODEL COMPARISONS TO DATA. .............. 88

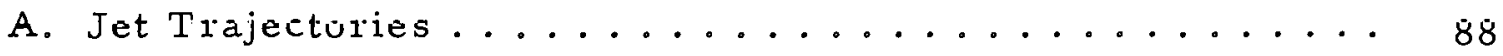

B. Centerline Temperature Decay and Temperature Half-

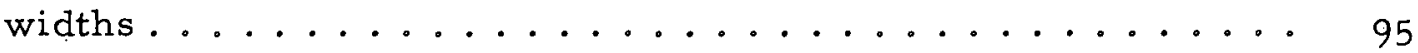

C. Centerline Velocity Decay and Velocity Half-widths...... 102

D. Temperature and Velocity Half-depth ........... 104

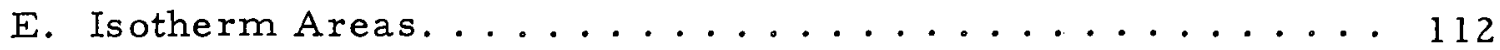

F. Decay of Centerline Temperature and Velocity with Depth . 115

G. Variation of Temperature and Velocity Width with Depth . . 119

VIII. SUMMARY AND CONCLUSIONS .................. 123

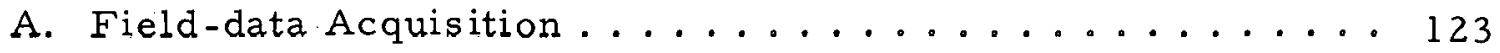

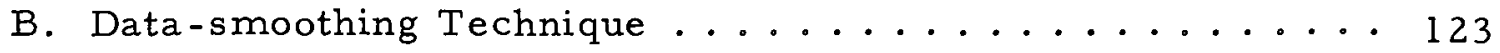


TABLE OF CONTENTS

$\underline{\text { Page }}$

C. Analytical Model; Field-data Comparisons. . . . . . . . . 124

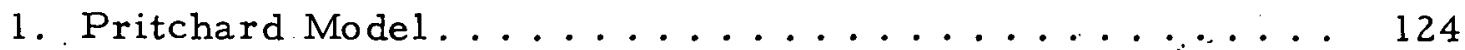

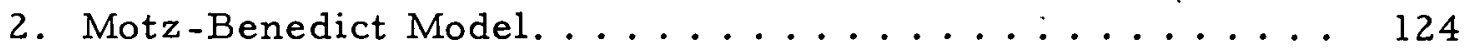

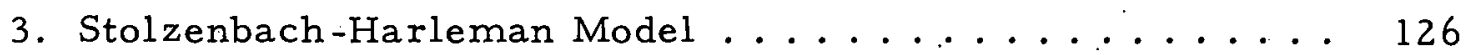

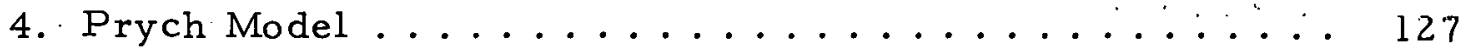

IX. RECOMMENDATIONS FOR FUTURE RESEARCH. . . . . . . . 128

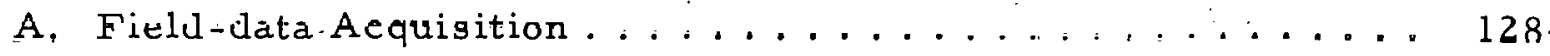

B. Data-smoothing 'l'echnique ........................ 128

C. Analytical Model; Field-data Comparisons .......... 128

APPENDIXES

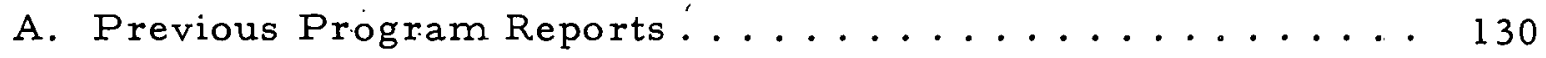

B. Preliminary Feasibility Study . . . . . . . . . . . 133

C. FORTRAN Listing for Fitting Procedure........... 138

D. Fitting Parameters and Results.............. 152

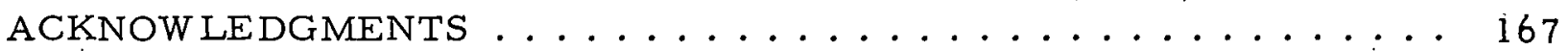

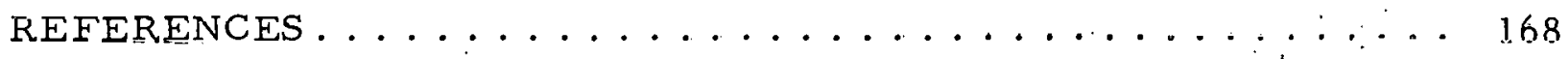




\section{LIST̈ OF FIGURES}

No.

Title

Page

1. Boat with Current Meter Used in Jet-regime Studies . . . . . . . 16

2. Motorola Mini-Ranger Range Positioning System. . . . . . . . 16

3. Bendix Q-15 Geomagnetic Ducted Current Meter. . . . . . . . 17

4. Aerial View of Point Beach Nuclear Power Plant. . . . . . . . . 20

5. Aerial View of Palisades Nuclear Power Plant ........... 21

6. Approximate Depth Contours near Point Beach Outfall . . . . . . 23

7. Approximate Depth Contours near Palisades Outfall. . . . . . . 24

8. Jet-regime Study for $0.5-\mathrm{m}$ Depth at Point Beach Power Plant (Unit 1): May 18,1972, 1115-1440 Hours .......... 25

9. Jet-regime Study for 1.0-m Depth at Point Beach Power Plant (Unit 1): May 18,1972,1115-1440 Hours.......... 26

10. Jet-regime Study for $1.5-\mathrm{m}$ Depth at Point Beach Power Plant (Unit 1): May 18,1972, 1115-1440 Hours . . . . . . . . 27

1.1. Jet-regime Study for 2.0 -m Depth at Point Beach Power Plant (Unit 1): May 18, 1972, 1115-1440 Hours. . . . . . . . 28

12. Jet-regime Study for 2.5 -m Depth at Point Beach Power Plant (Unit 1): May 18,1972,1115-1440 Hours.......... 29

13. Jet-regime Study for 3.0-m Depth at Point Beach Power Plant (Unit 1): May 18, 1972, 1115-1440 Hours ..........

14. Jet-regime Study for $0.5-\mathrm{m}$ Depth at Point Beach Power Plant (Unit 1): May 23, 1972, 0945-1700 Hours ......... 31

15. Jet-regime Study for 1.0 -m Depth at Polnt Beach Pover Plant (Unit 1): May 23, 1972, 0945-1700 Hours ..........

16. Jet-regime Study for 1.5-m Depth at Point Beach Power Plant (Unit 1): May 23, 1972,0945-1700 Hours..........

17. Jet-regime Study for $2.0 \mathrm{-m}$ Depth at Point Beach Power Plant (Untt 1): May 23, 1972, 0945-1700 Hours......... 34

18. Jet-regime Study for $2.5-\mathrm{m}$ Depth at Point Bearh Power Plant (Unit 1): May 23,1972,0945-1700 Hours ..........

19. Jet-regime Study for $3.0-\mathrm{m}$ Depth at Point Beach Power Plant (Unit 1): May 23, 1972, 0945-1700 Hours.......... 36

20. Jet-regime Study for $0.5-\mathrm{m}$ Depth at Point Beach Power Plant (Unit 1): July 13, 1972, 1308-1706 Hours.......... 37 


\section{LIST OF FIGURES}

No

Title

$\underline{\text { Page }}$

21. Jet-regime Study for $1.0-\mathrm{m}$ Depth at Point Beach Powe $\mathrm{r}$

Plant (Unit 1): July 13,1972,1308-1706 Hours.........

22. Jet-regime Study for $1.5-\mathrm{m}$ Depth at Point Beach Power

Plant (Unit 1): July 13, 1972, $1308-1706$ FIours . .........

23. Jet-regime Study for 2.0-m Depth at Point Beach Power

Plant (Unit 1): July 13,1972,1308-1706 Hours . ........ 40

24. Jet-regime Study for 2.5-m Depth at Point Beach Power

Plant (Unit 1): July 13,1972, 1308-1706-Hours ......... 41

25. Jet-regime Study for $3.0-\mathrm{m}$ Depth at Point Beach Power

Plant (Tnit 1): July 13,1972,1308-1706 Hours..........

26. Jet-regime'Study for 0.5-m Depth at Point Beach Power

Plant (Unit 1): September 9, 1972, 1045-1420 Hours .......

27. Jet-regime Study for 1.0-m Depth at Point Beach Power

Plant (Unit 1): September 9, 1972, 1045-1420 Hours .......

28. Tet-regime Study for $1.5-\mathrm{m}$ Depth at Point Beach Power

Plant (Unit 1): September 9, 1972, 1045-1420 Hours .......

29. Jet-regime Study. for $2.0-\mathrm{m}$ Depth at Point Beach Power

Plant (Unit 1): September 9, 14\%2, 1045-1420 Hours .......4 46

30. Tet-regime Study for $2.5 \mathrm{-m}$ Depth at Point Beach Power

Plant (Unit 1): September 9,1972, 1045-1420 Ilour3 .......

31. Jet-regime Study for 3.0 - $m$ Depth at Point Beach Power

Plant (Unit 1): September $9,1972,1045-1420$ Hours .......

32. Jet-regime Study for $0.5-\mathrm{m}$ Depth at Palisades Power Plant:

.Tune 14,1972, 1000-1348 Hours ...............

33. Tet-regime Study for $1.0-\mathrm{m}$ Depth at Palisades Power Plant:

June $14,1972,1000-1348$ Hours . ...............

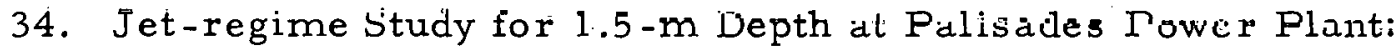

June $14,1972,1000-1348$ IIour $\ldots \ldots \ldots$

35. Jet-regime Study for $2.0-\mathrm{m}$ Depth at Palisades Power Plant:

June 14, 1972, 1000-1348 Hours ...............

36. Jet-regime Study for $2.5-\mathrm{m}$ Depth at Palisades Power Plant:

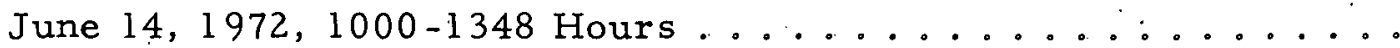

37. Jet-regime Study for $0.5-\mathrm{m}$ Depth at Palisades Power Plant:

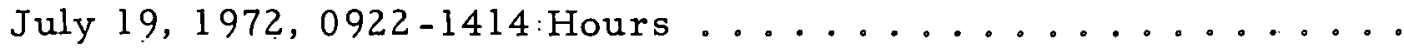




\section{LIST OF FIGURES}

No.

Title

$\underline{\text { Page }}$

38. Jet-regime Study for 1.0-m Depth at Palisade's Power Plant:

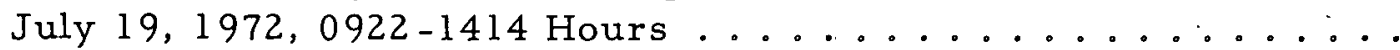

39. Jet-regime Study for $1.5 \mathrm{-m}$ Depth at Palisades Power Plant:

July 19, 1972, $0922-1414$ Hours $\ldots \ldots \ldots 6$

40. Jet-regime Study for $2.0-\mathrm{m}$ Depth at Palisades Power Plant:

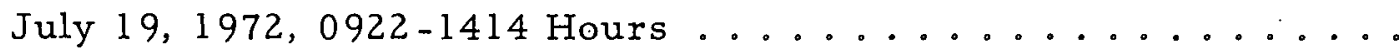

41. Jet-regime Study for 2.5-m Depth at Palisades Power Plant:

July 19, 1972, 0922-1414 Huurs ..............

42. Jet-regime Study for 3.0-m Depth at Palisades Power Plant:

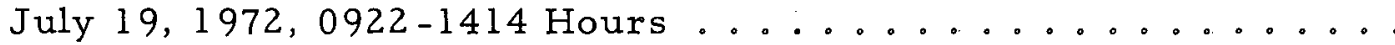

43. Jet-regime Study for $0.5-\mathrm{m}$ Depth at Palisades Power Plant:

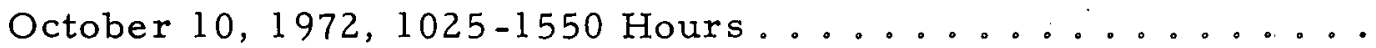

44. Jet-regime Study for 1.0-m Depth at Palisades Power Plant:

October 10,1972, 1025-1550 Hours ..............

45. Jet-regime Study for $1.5-\mathrm{m}$ Depth at Palisades Power Plant:

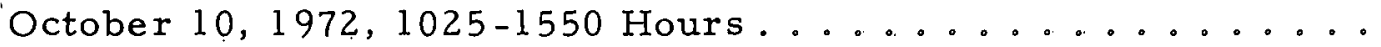

46. Jet-regime Study for $2.0-\mathrm{m}$ Depth at Palisades Power Plant:

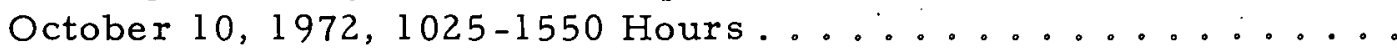

47. Jet-regime Study for $2.5-\mathrm{m}$ Depth at Palisades Power Plant:

October 10,1972, 1025-1550 Hours .............

48. Jet-regime Study for $3.0 \mathrm{-m}$ Depth at Palisades Power Plant:

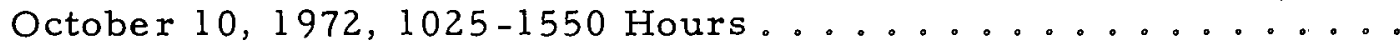

49. Center rine Excess-temperature and -velocity Decays

Resulting from Fits to Point Beach Jet Data at 0.5-m Depth ...

50. Half-widths of Temperature and Velocity Distributions

Resulting from Fits to Point Beach Jet Data at $0.5-\mathrm{m}$ Depth ... . 70

51. Centerline Temperature Excess and Velocity Excess as a

Function of Depth Resulting from Fits to Point Beach Jet Data. . .

52. Half-widths of Temperature and Velocity Distributions as a

Function of Depth at $s=150 \mathrm{~m}$, Resulting from $\mathrm{F}^{\prime}$ its to

Point Beach Jet Data .....................

53. Geometrical Characteristics of Jet Assumed in Stolzenbach-

Harleman Model .......................

54. Velocity and Temperature Characteristics of Jet Assumed in Stolzenbach-Harleman Model.................. 
LIST OF FIGURES

No

Title

Page

55. Definition Sketch for Coordinate System and Jet Region

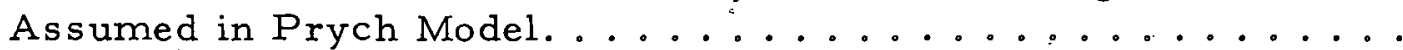

56. Centerline Trajectories Resulting from the Fitting Procedure and Model Calculations for Point Beach: May 18, 1972......990

57. Centerline Trajectories Resulting from the Fitting Procedure and Model Calculations for Point Beach: May 23, 1972......

58. Centerline Trajectories Resulting from the Fitting Procedure and Model Calculations for Point Beach: July 13,1972......

59. Centerline Trajectories Resulting from the Fitting Procedure and Model Calculations for Point Beach: September 9, 1972 ...

60. Centerline Trajectories Resulting from the Fitting Procedure and Model Calculations for Palisades: October 10, 1972.....

61. Idealized Surface Profile of Excess 'lemperature $\theta / \theta_{0}$, and Velocity $u / u_{0}$ across Bent Jet near Orifice ............

62. Bottom Depth at Palisades Outfall for Three Dates of

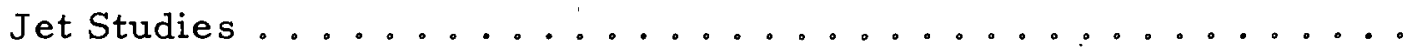

63. Centerline Temperature Excess and Velocity Decays Resulting from the Fitting Procedure and Mudel Calculations for

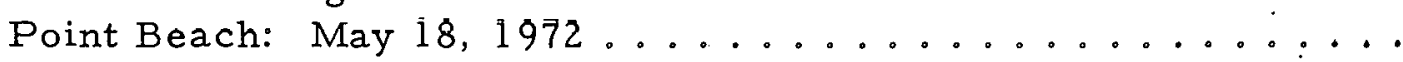

64. Centerline Temperature Excess and Velocity Decays Resulting from the Fitting Procedure and Model Calculations for

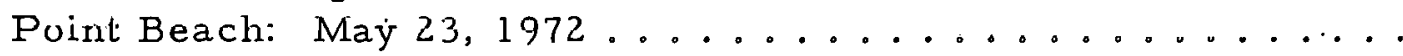

65. Centerline Temperature Excess and Velocity Decays Resulting from the Fitting Procedure and Model Calculations for

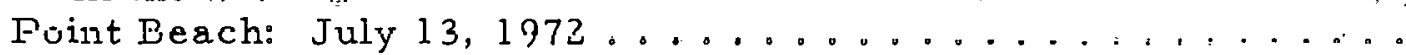

66. Centerline Temperature Excess and.Velocity Decays Resulting from the Fitting Procedure and Model Calculations for Polnt Bedch: Seplenilue $9,1772 \ldots \ldots \ldots$

67. Centerline Temperature Excess and Velocity Decays Resulting from the Fitting Procedure and Model Calculations for Palisades: October 10,1972................

68. Half-widths of Temperature and Velocity Distributions Resulting from the Fitting Procedure and Model Calculations for Point Beach: May 18,1972................

69. Half-widths of Temperature and Velocity Distributions Resulting from the Fitting Procedure and Model Calculations

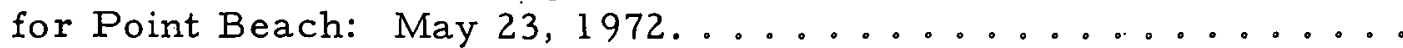




\section{LIST OF FIGURES}

No. $\underline{\text { Title }}$

70. Half-widths of Temperature and Velocity Distributions Resulting from the Fitting Procedure and Model Calculations for Point Beach: July 13,1972. . . . . . . . . . . . 100

71. Half-widths of Temperature and Velocity Distributions Resulting from the Fitting Procedure and Model Calculations for Point Beach: September 9,1972 . . . . . . . . . .

72. Half-widths of Temperature and Velocity Distributions Resulting from the Fitting Procedure and Model Calculations for Palisades: October 10,1972............... 101

73. Half-depths of Temperature and Velocity Distributions Resulting from Model Calculations for Point Beach: May 18, 1972. . . . . . . . . . . . . . . . . 104

74. Half-depths of Temperature and Velocity Distributions Resulting from Model Calculations for Point Beach: May 23, 1972........................ 104

75. Half-depths of Temperature and Velocity Distributions Resulting from Model Calculations for Point Beach:

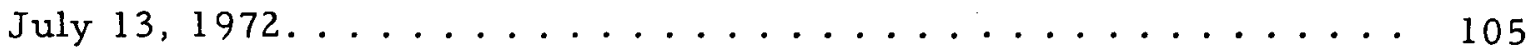

76. Half-depths of Temperature and Velocity Distributions Resulting from Model Calculations for. Point Beach: September 9, $1972 \ldots \ldots \ldots \ldots 105$

77. Half-depths of Temperature and Velocity Distributions Resulting from Model Calculations for Palisades:

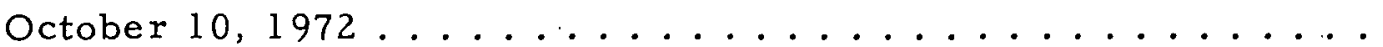

78. Is otherm Areas Resulting from the Fitting Procedure and Model Calculations for. Point Beach: May 18,1972 .......113

79. Isotherm Areas Resulting from the Fitting Procedure and Model Calculations for Point Beach: May 23, 1972 . . . . . 113

80. Isotherm Areas Resulting from the Fitting Procedure and Model Calculations for Point Beach: July 13, $1972 \ldots \ldots \ldots \ldots 114$

81. Isotherin Areas Resulting from the Fitting Procedure and Model Calculations for Point Beach: September 9, 1972. . . . 114

82. Isotherm Areas Resulting from the Fitting Procedure and Model Calculations for Palisades: October 10,1972 ...... 115

83. Idealized Decay of Temperature Excess and Velocity Excess with Depth ............................ 116 


\section{LIST OF FIGURES}

No

Title

Page

84. Centerline Temperature Excess and Velocity Excess as a Function of Depth at $150 \mathrm{~m}$ from Outfall Resulting from the Fitting Procedure and Model Calculations for Point Beach:

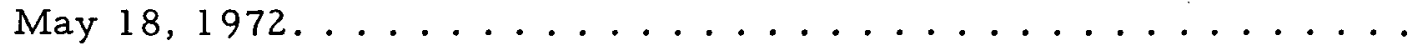

85. Centerline Temperature Excess and Velocity Excess as a Function of Depth at $150 \mathrm{~m}$ from Outfall Resulting from the Fitting Procedure and Model Calculations for Point Beach: May 23.1976. . . . . . . . . . . . . . . . .

86. Centerline Temperature Excess and Velocity Excess as a Function of Dcpth at $150 \mathrm{~m}$ from Outfall Rosulting from the Fitting Procedure and Model Calculations for Point Beach:

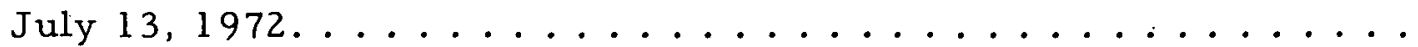

87. Centerline Temperature Excess and Velocity Excess as a Function of Depth at $150 \mathrm{~m}$ from Outfall Resulting from the Fitting Procedure and Model Calculations for Point Beach:

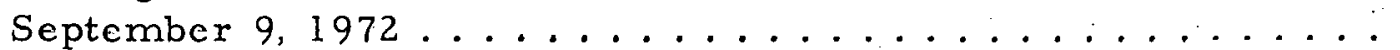

88. Centerline Temperature Excess and Velocity Excess as a Function of Depth at $150 \mathrm{~m}$ from Outfall Resulting from the Fitting Procedure and Model Calculations for Palisades:

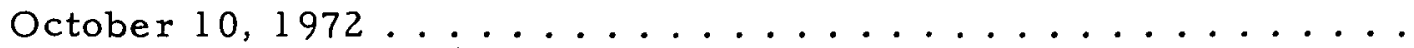

89. Half-widths of Temperature and Velocity Distributions as a Function of Depth at $150 \mathrm{~m}$ from Outfall Resulting from the Fitting Procedure and Model Calculations for Point Beach:

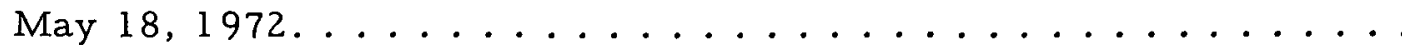

90. Half-widths of Temperature and Velocity Distributions as a Function of Depth at $150 \mathrm{~m}$ from Outfall Resulting from the Fitting Procedure and Model Calculations for Point Beach: May 23, 1972....................... 120

91. Half-widths of Temperature and Velocity Distributions as a Function of Depth at $150 \mathrm{~m}$ from Outfall Resulting from the Fitting Procedure and Model Calculations for Point Beach: July $13,1972 \ldots \ldots \ldots \ldots \ldots \ldots \ldots \ldots$

92. Half-widths of Temperature and Velocity Distributions as a Function of Depth at $150 \mathrm{~m}$ from Outfall Resulting from the Fitting Procedure and Model Calculations for Point Beach:

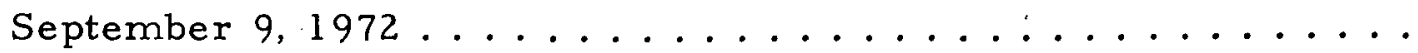

93. Half-widths of Temperature and Velocity Distributions as a Function of Depth at $150 \mathrm{~m}$ from Outfall Resulting from the Fitting Procedure and Model Calculations for Palisades: October 10,1972 ..................... 


\section{LIST OF FIGURES}

No.

Title

$\underline{\text { Page }}$

94. Station Locations for Jet-regime Study: November 3, 1971 ,

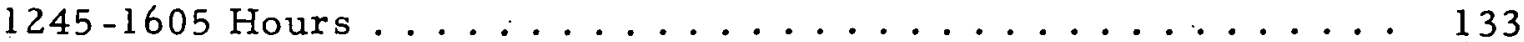

95. Jet-regime Study for 2 -ft Depth at Point Beach Power Plant

(Unit 1): November 3,1971, 1245-1605 Hours........... 134

96. Jet-regime Study for 4 -ft Depth at Point Beach Power Plant

(Unit 1): November 3, 1971, 1245-1605 Hours.......... 135

97. Jet-regime Study for 6 -ft Depth at Point Beach Power Plant

(Unit 1): November 3,1971, 1245-1605 Hours.......... 136 
I. Instrument Accuracy. .................. 18

II. Summary of Characteristics of Jet Models . . . . . . . . . 75

III. Summary of Characteristics of Complete-field Models . . . . . 76

IV. Data and Parameters Used for Model Calculations for

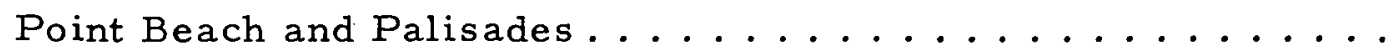

V. Determination of Temperature and Velocity Half-depths at Each Station Location from Point Beach Data of May 18, 1972 . . 107

VI. Determination of Temperature and Velocity Half-depths at Each Station Location from Point Beach Data of May 23, 1972 , 108

VII. Determination of Temperature and Velocity Half-depths at Each Station Location from Point Beach Data of July 13, 1972 . 109

VIII. Determination of Temperature and Velocity Half-depths at Each Station Location from Point Beach Data of

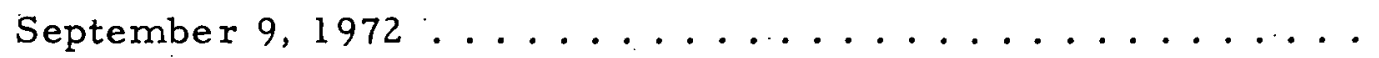

IX. Determination of Temperature and Velocity Half-depths at Each Station Location from Palisades Data of October 10, 1972 . 


\section{TEMPERATURE AND VELOCITY MEASUREMENTS AND PREDICTIVE MODEL COMPARISONS \\ IN THE NEAR-FIELD REGION OF SURFACE THERMAL DISCHARGES}

R. A. Paddock, A. J. Policastro, A. A. Frigo, D. E. Frye, and J. V. Tokar

\section{ABSTRACT}

Simultaneous temperature and velocity measurements were made in the near-field region of the surface thermal discharge at the Point Beach Unit 1 and Palisades Nuclear Power Plants on Lake Michigan. Data collected include measurements of temperature and velocity at the $0.5-, 1.0-, 1.5-, 2.0-, 2.5-$, and $3.0-\mathrm{m}$ depths, along with measurements of ambient lake and meteorological conditions. Bottom depth was also measured at various locations. Four such surveys were made at the Point Beach plant, three at the Palisades plant.

To examine the jet features from the above surveys and facilitate a comparison with analytical model predictions, a computer program was written to smooth the data, extracting such jet characteristics as trajectory, centerline temperature decay and temperature half-widths, centerline velocity decay and velocity half-widths, temperature and velocity half-depths, and isotherm areas.

Four neàr-field analytical models often used in environmental impact evaluations of power-plant surface discharges are compared to the jet characteristics determined from the smoothed jet data. The Pritchard model compares rather well with these limited data and is often conservative when model-data discrepancies exist. The Stolzenbach-Harleman and Prych models predict too rapid a temperature and velocity decay accompanying too great a lateral spread. The Motz-Benedict model is too sensitive to an entrainment coefficient $E$ with little consistent data available for its determination for accurate prediction.

Recommendations for future research, encompassing the field-data acquisition, the smoothing procedure, and the presently available models are included. 


\section{INTRODUCTION}

Under the auspices of the U.S. Atomic Energy Commission, the Argonne Center for Environmental Studies has been studying the physical effects of heated condense $r$ discharges from steam-electric power plants on the Great Lakes since FY 1970. Appendix A lists the reports published under this program to the present. Two of the primary objectives of this program have been and continue to be the acquisition of reasonably complete prototype thermal-plume field data and the verification of analytical predictive plume models. To this end, field data in the jet regime of two nuclear power plants on Lake Michigan have been collected and compared to models, with the results reported herein.

The jet regime (the near field) is that region of the discharge in which the heated effluent enters the receiving body of wate $r$ possessing a velocity and temperature disparity with respect to the receiving body. Thus, as a heated effluent enters an ambient environment from a particular plant outfall, viscous shear between the effluent and the ambient fluid creates turbulence in the contact region. This turbulence works its way both inward toward the jet centerline and outward toward the ambient fluid, with a resultant net outward flux of momentum and heat away from the jet axis. Within this regime of the discharge, it is the mechanical mixing action induced by the kinetic energy of the discharge itself that dominates the eddy transport mechanisms. At some distance from the outfall, the kinetic energy of the discharge will be sufficiently dissipated to allow the natural turbulence existing within the ambient receiving water, together with buoyant forces, to dictate plume dispersion. It is nominally assumed that the effluent is no longer jetlike in character when this situation is reached.

From a regulatory point of view, the jet regime is of particular interest. It is often within this region that outfall architects must design their discharges to meet thermal water-quality criteria that limit the temperature rise in the thermal plume beyond a prescribed distance from the point of discharge. This is commonly referred to as a mixing-zone limitation. Some states have adopted very restrictive mixing-zone criteria; others have none at all. 'Therefore, depending on the nature of the receiving body, the size of plant, and a multitude of different factors including the thermal criteria, each plant outfall design is more or less tailored to the particular siting situation. On the Great Lakes, the predominant outfall design happens to be a shoreline, open, rectangular discharge canal. Several more recent plant designs have used more sophisticated offshore multiorifice submerged discharges, called diffusers.

The literature contains numerous models that attempt to predict the behavior of shoreline canal discharges. Some of these models are qualitative in nature; others profess to be quantitative as well. One thing all these models have in common is that none has been generally verified with prototype field data. To compound the problem, a survey of the literature reveals surprisingly little actual jet-regime field data with which models can be tested. Since there 
is such a paucity of data in the jet regime, a field program was developed by the Center for Environmental Studies specifically to acquire prototype data near canal-type discharges. This program has been partially described in Refs. 21 and 25 of Appendix A. A description of experimental methods and detailed information concerning the results of seven jet studies obtained during 1972 are presented herein.

Since it would have been difficult to compare the field data directly to results obtained from analytical models, a data-smoothing technique was developed to help in this endeavor. The smoothing method was primarily developed to glean as much information from the experimental data as possible, considering the limited number of data points collected using the present field technique. A complete description of the smoothing method appears in Sec. V. While one should recognize that the smoothing method has some obvious limitations and biases, it nevertheless has worked out quite well for the purposes for which it was designed.

Lastly, the results of the smoothing procedure are compared to four analytical models that have been used, in some cases quite extensively, for predictive purposes. These comparisons and a discussion of them appear in Sec. VII. Note that the success or apparent lack of success displayed by a particular model should not, at this point, be considered as a total test of the model. Many more data comparisons under different outfall situations must be made before any model can be realistically evaluated.

In summary, this report brings together details concerning the acquisition, smoothing, and model analysis of a relatively unique set of jet-regime plume field data. We hope this report will stimulate more interest than has been shown in the literature in attempting to validate existing predictive models. In our opinion, too many predictive models existing in the literature have not been adequately tested. Much reliable field data is just now becoming available, and it should be the immediate goal of those interested in applying predictive models to test these models with actual field data. Only in this way can we hope for a positive improvement in the existing state of the art. 


\section{EXPERIMENTAL TECHNIQUE}

A two- or three-point mooring system was used to hold Argonne's $5 \frac{1}{2}-\mathrm{m}(18-\mathrm{ft})$ cathedral-hull fiberglass boat, the R. V. Aha, steady while ob-

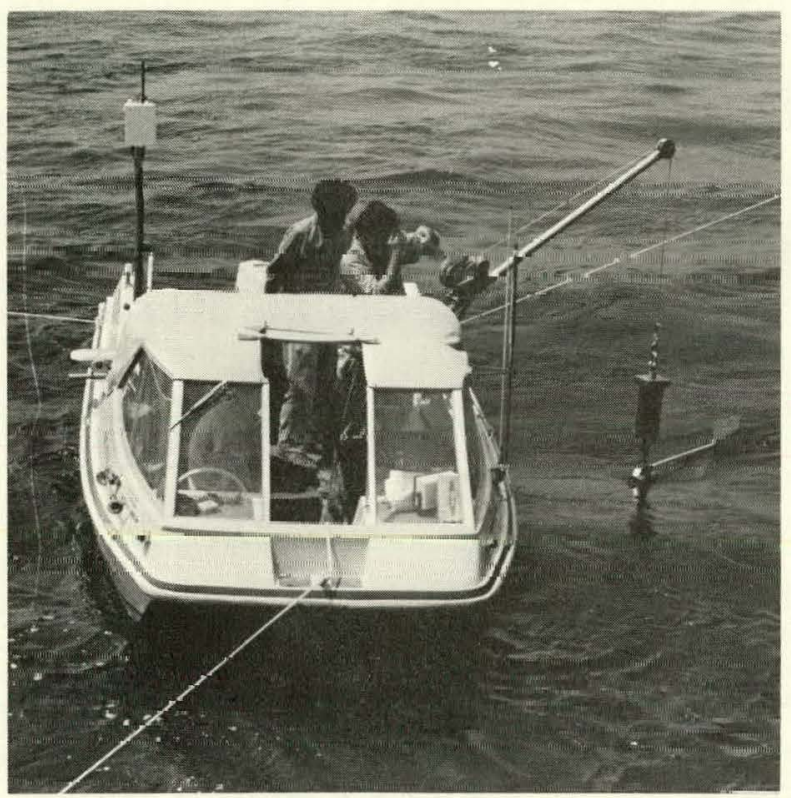

Fig. 1. Boat with Current Meter Used in Jet-regime Studies taining simultaneous temperature and velocity measurements in the nearfield region of the thermal plume (see Fig. 1). Anchors were located on either side of the plume, and for regions very near the outfall, a third line was somctimes attached to the ninttall itself. Transects across the plume centerline were then made at various distances from the outfall. The position of the boat was held relatively constant at the various measuring stations, and the position of each station was determined by using a Motorola Mini-Ranger range positioning system (Fig. 2). This positinning system cunsists of two shorc-based transponders with a receiver-transmitter unit and range console on board the boat that displays the range information

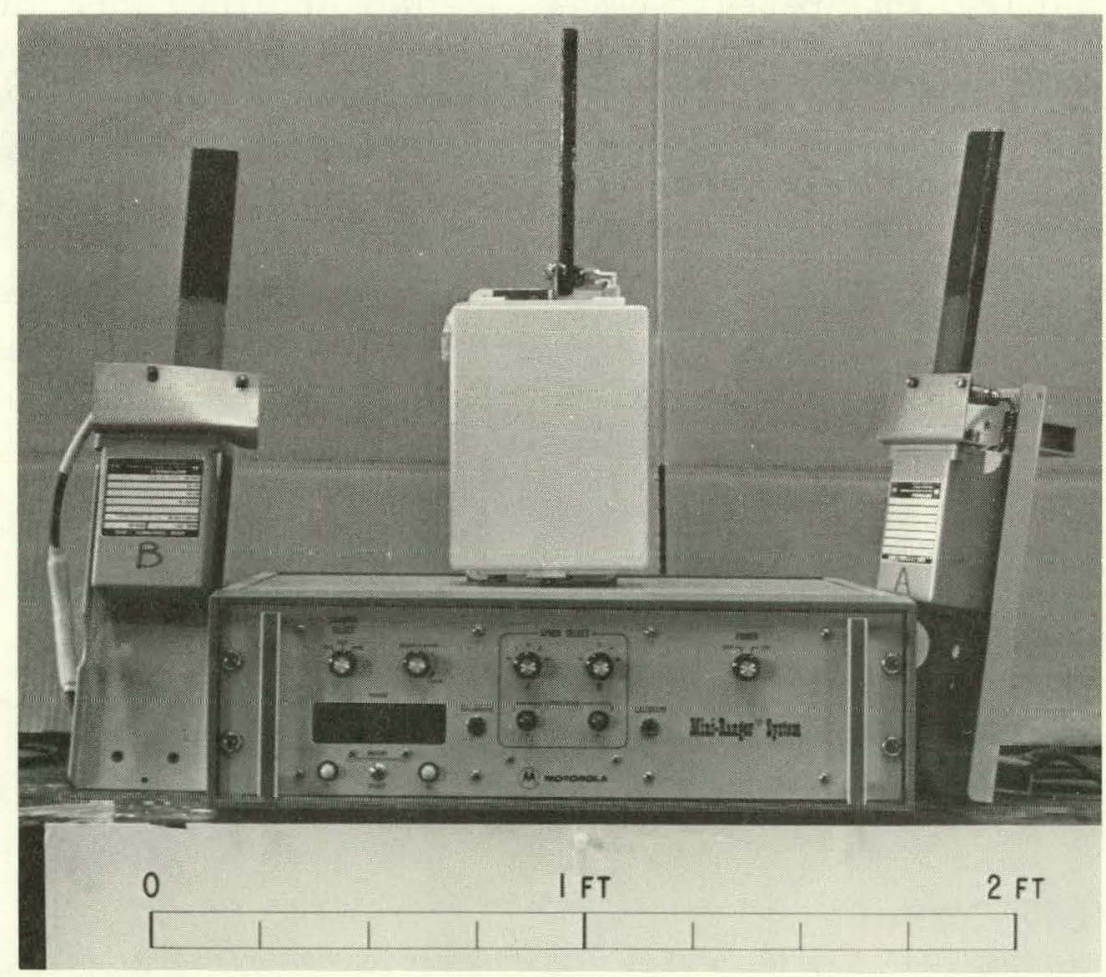

Fig. 2. Motorola Mini-Ranger Range Positioning System 
from each transponder. The boat's position can then be found by trilateration. The Mini-Range $\mathrm{r}$ is powered by $110 \mathrm{~V}$ ac (available from a 24-V dc highefficiency Flitetronics PC 16 Air-

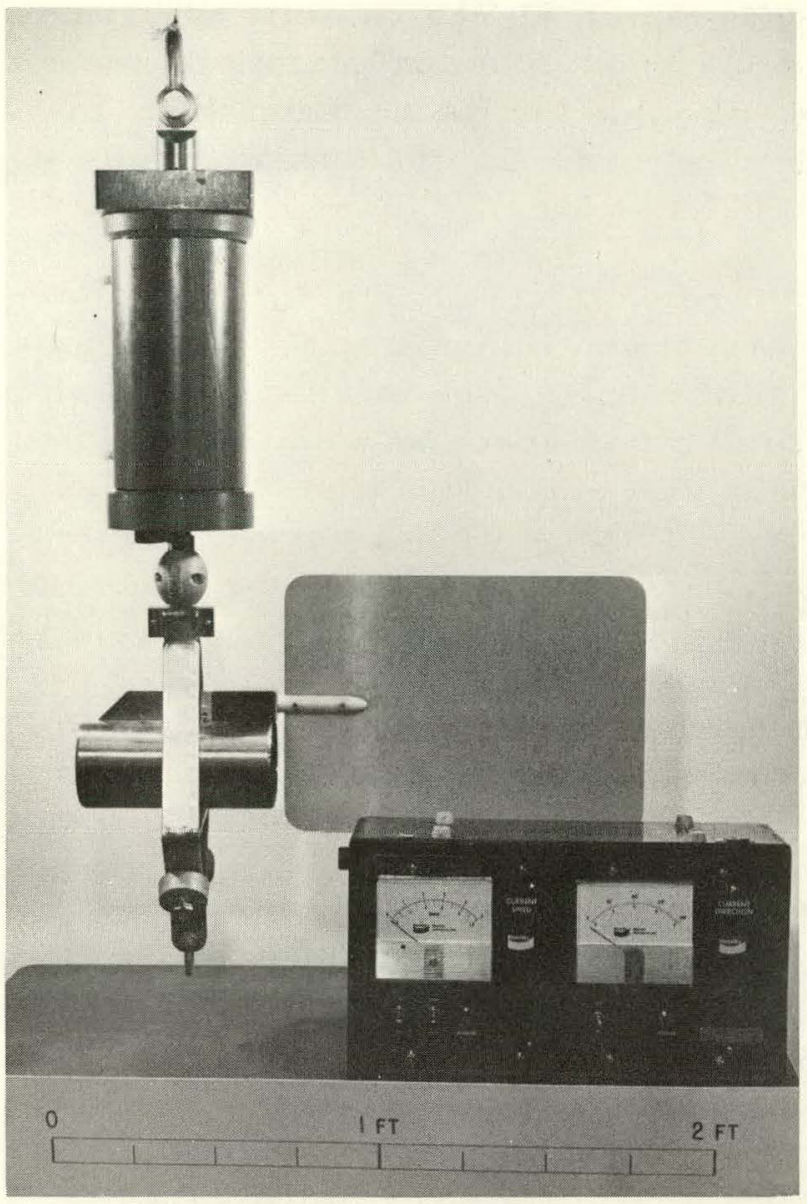

Fig. 3. Bendix Q-15 Geomagnetic Ducted Current Meter. ANL Neg. No. 190-568-11. craft Static Inverter). The usable range of the system with omnidirectional antennas is about $16 \mathrm{~km}$.

\section{A Bendix Q-15 geomagnetic} ducted current meter (Fig. 3), with an attached YSI thermistor, was used to measure the velocity and temperature of the discharge waters. The Q-15 has a five-bladed impeller that rotates in both directions and is enclosed in a duct. The ducted assembly is aligned with the current by a vane of adjustable length. The effects of wave and boat motion are nulled out by electronic averaging (over about $25 \mathrm{sec}$ ) of the number of turns of the impeller and by the presence of the duct. Current speed and direction are displayed on deck by means of a readout unit, Bendix Model No. S-232, which is connected to the current meter through a four-conductor cable. The current meter is powered by six $9-\mathrm{V}$ batteries. The meter was lowered over the side of the boat and suspended at $0.5-\mathrm{m}$ intervals to a depth of $3.0 \mathrm{~m}$ or to the bottom. The first time the experiment was being conducted, it was discovered that time variations in velocity and temperature occur. Thus, in order to obtain average values of velocity and temperature along with any variation, strip chart recorders were connected to the currentmeter and thermistor outputs.

Note at this point that a variety of factors inherent in making measurements in the jet regime may cause uncertainties in the data. These problems must be understood if proper use is to be made of the data.

An important aspect of the experimental uncertainty is the short-term variations in the velocity and temperature of the discharge jet (see Ref. 30). The cause of these variations is not clear at present, but they may be due to eddies created at the interface between the jet and the ambient water, to surging which is apparent in the discharge canal, or to other factors. These fluctuations appear to have periods ranging from a few seconds to a few minutes. 
Since point-by-point measurements in the jet were typically made over a period of 1-2 $\mathrm{min}$, it is apparent that an unrepresentative value might be obtained at a given point. The scale of the short-term temperature fluctuations is on the order of several Centigrade degrees or less; the velocity variations are on the order of $50 \%$ of the mean value or less. These fluctuations were not present at all locations. A more typical value for the uncertainty in the temperature measurement is $\pm 0.5 \mathrm{C}^{\circ}$; a typical value for the uncertainty in the current speed is $\pm 20 \%$.

Another source of uncertainty, in terms of data analysis, is the ambiguity attached to the values of ambient current and temperature. These numbers, necessarily assumed to be constants throughout the measurement (which lasted from 3 to $7 \mathrm{hr}$ ), vary not only in time but in space as well. Ambientcurrent measurements were typically made at a single location (at several depths) before and after the jet-regime measurements. Here again, current fluctuations in time and position may lead, for a variety of reasons, to an unrepresentative value for ambient-current speed. (Direction of the current is thought to be more definite.) Ambient-current speeds as reported may have an uncertainty of 20-50\%; lower current speeds are the most uncertain. On some occasions, ambient-temperature measurements are as difficult to pin down as ambient-current speed and are somewhat more important in terms of the analysis to be described. We chose the ambient temperature to be the water temperature (at the appropriate depth), which appeared not to be influenced by the discharge water, yet was in the vicinity of the discharge. Unfortunately, on days when upwelling, downwelling, shoreline heating, or other disturbing phenomena occurred, the reported values of ambient water temperature may have an uncertainty of as much as $1-2 \mathrm{C}^{\circ}$.

In the face of the previously discussed uncertainties in the data, instrumentation accuracy may not be very important, but for completeness, Table I lists the instrument specifications. Of special interest in terms of velocity measurements is the fact that, while a ducted current meter is used to null out disturbing vertical motion, shielding of the impeller occurs if the duct is not aligned with the flow. The importance of this remains unclear, but because the meter continuously averages the speed over a 25-sec period, any shielding would result in lower values for current speed.

\begin{tabular}{|c|c|c|c|c|c|c|c|}
\hline Instrument & Sensor & Accuracy & Threshold & Range & Time Constant & Resolution & Remarks \\
\hline \multirow[t]{2}{*}{$\begin{array}{l}\text { Bendix Q-15 } \\
\text { Current Meter }\end{array}$} & Speed; impeller & $\begin{array}{l} \pm 4 \% \text { of } \\
\text { full scale }\end{array}$ & $3 \mathrm{~cm} / \mathrm{sec}$ & $\begin{array}{l}0-1.0 \text { knot: low scale } \\
0-5.0 \text { knots: high scale }\end{array}$ & $\begin{array}{l}25 \mathrm{sec} \text { : low scale } \\
2.5 \mathrm{sec} \text { : high scale }\end{array}$ & $\begin{array}{l}1 \mathrm{~cm} / \mathrm{sec} \text {; low scale } \\
5 \mathrm{~cm} / \mathrm{sec} \text { : high scale }\end{array}$ & \\
\hline & $\begin{array}{l}\text { Direction: compass } \\
\text { with vane }\end{array}$ & \pm 120 & - & $0-360^{\circ}$ & - & 50 & Vane has adjustable length: $03-30 \mathrm{~m}$ \\
\hline $\begin{array}{l}\text { Temperature } \\
\text { Recorderd }\end{array}$ & Thermistor & $\pm 0.5 \mathrm{C}^{\circ}$ & - & $0-30^{\circ} \mathrm{C}$ & $\sim 2.5 \mathrm{sec}$ & $0.2 C^{\circ}$ & $\begin{array}{l}\text { Consisting of a Rustrak Model No. } 2133 \\
\text { temperature recorder and YSI No. } 409 \\
\text { thermistor probe }\end{array}$ \\
\hline $\begin{array}{l}\text { Temperature } \\
\text { Recorder } b\end{array}$ & Thermistor & $\pm 0.2 \mathrm{C}^{\circ}$ & - & $0-50^{\circ} \mathrm{C}$ & $\sim 2.5 \mathrm{sec}$ & $0.1 c^{\circ}$ & $\begin{array}{l}\text { Consisting of YSI No. } 709 \text { probe and } \\
\text { digital readout built at Argonne }\end{array}$ \\
\hline $\begin{array}{l}\text { Motorola } \\
\text { Mini-Ranger }\end{array}$ & - & $\pm 3 \mathrm{~m}$ & - & $0.1-35 \mathrm{~km}$ & - & $1 \mathrm{~m}$ & $\begin{array}{l}\text { Accuracy applies to each range meas- } \\
\text { urement. System accuracy varies with } \\
\text { position of transponders relative to boat. } \\
\text { For most of these measurements, } \pm 3 \mathrm{~m} \\
\text { would apply. }\end{array}$ \\
\hline
\end{tabular}

aUsed in temperature measurements up to and including July 19. 1972.

bused in temperature measurements after July 19. 1972. 


\section{DESCRIPTIONS OF POWER PLANTS}

The jet regimes of the thermal plumes were surveyed at two power plants located on Lake Michigan. The power plants studied were the Point Beach Nuclear Power Plant, operated by the Wisconsin Electric Power Company and the Wisconsin Michigan Power Company, and the Palisades Nuclear Power Plant, operated by Consumers Power Company. Brief descriptions of these plants follow.

\section{A. Point Beach Nuclear Power Plant}

The Point Beach Nuclear Power Plant is in the town of Two Creeks, Wisconsin, on the western shore of Lake Michigan. (Figure 4 is an aerial view of the plant.) The plant is a two-unit steam-generating station. The nuclear reactors for each unit are pressurized light-water-moderated and - cooled systems. Each unit has a gross capacity of $523 \mathrm{MWe}$ and a net capacity of $505 \mathrm{MWe}$. The water intake for the plant consists of a circular crib $533 \mathrm{~m}$ from the shore. Cooling water for the operation of the power plant is drawn from Lake Michigan and passes through the cooling condensers at a maximum rate of $25.1 \mathrm{~m}^{3} / \mathrm{sec}$ for full-power operation of each individual unit of the plant. The water is returned to the lake about $50 \mathrm{~m}$ offshore through two 10.7-m-wide discharge flumes (one flume per unit). Water depth in the flumes is about $4.2 \mathrm{~m}$. During most of the field year, the second unit was not operational. Late in the summer, however, the second unit was operating at about $12 \%$ power and $50 \%$ of its rated discharge flow.

B. Palisades Nuclear Power Plant

The Palisades Nuclear Power Plant is near the city of South Haven, Michigan, on the eastern shore of Lake Michigan. (Figure 5 is an aerial view of the plant.) This plant uses a pressurized-water reactor to produce a maximum gross output of $714 \mathrm{MWe}$. During the 1972 field year, the plant was operaling at a net generating capacity of about $420 \mathrm{MWe}$. The cooling water is taken from Lake Michigan through an intake crib located $6.1 \mathrm{~m}$ below the lake's surface, $1.8 \mathrm{~m}$ from the lake bottom, and $1000 \mathrm{~m}$ from the shoreline. For the Palisades plant, as presently constructed, the cooling water passes through the cooling condenser at a maximum flow rate of $25.6 \mathrm{~m}^{3} / \mathrm{sec}$ and is returned to Lake Michigan, via a 32.9-m-long discharge canal at the shoreline. The canal is $11.3 \mathrm{~m}$ wide at the shoreline outlet and diverges to a width of $28.3 \mathrm{~m}$ at the point of discharge. At this point, the water has an average depth of about $2.1 \mathrm{~m}$. 


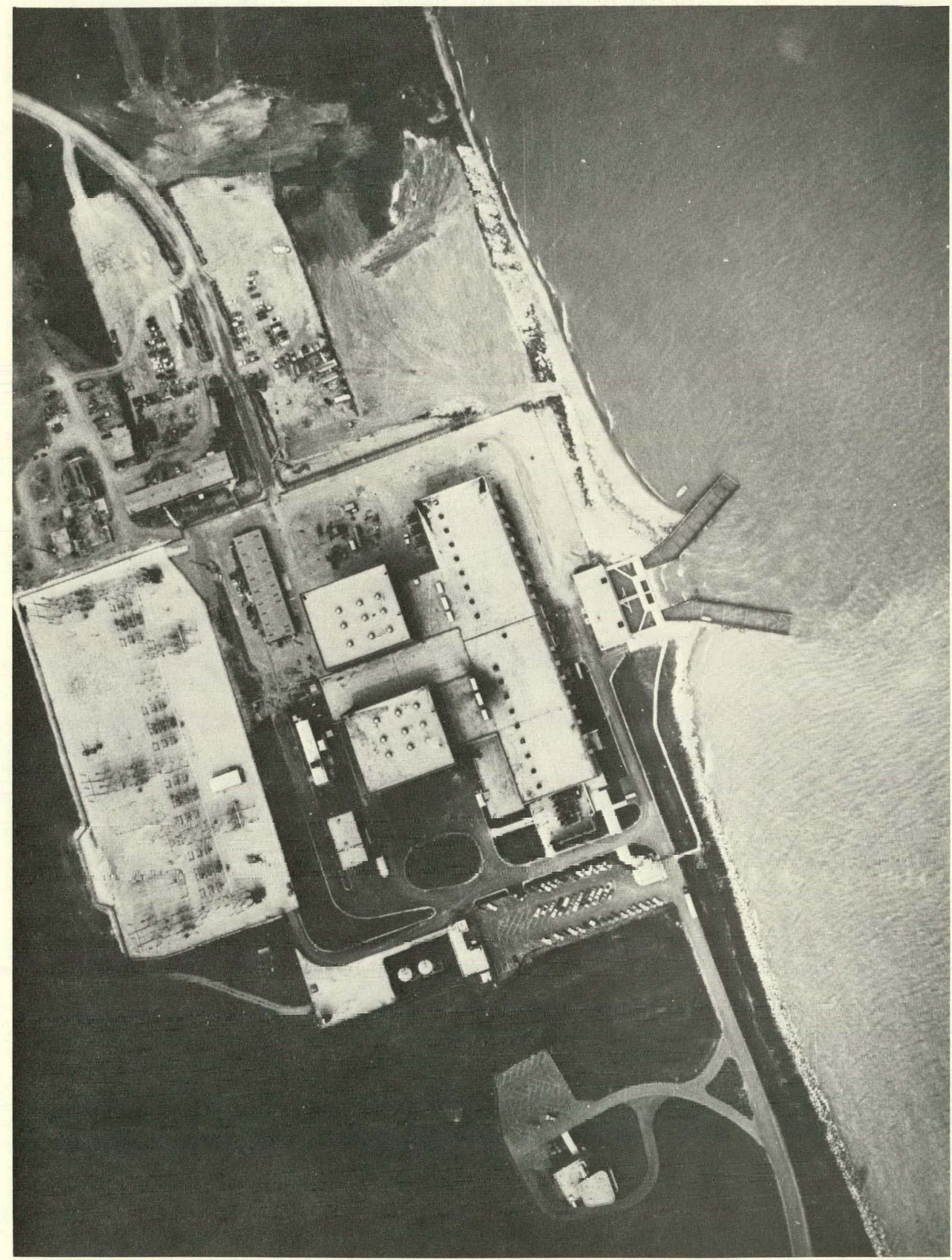

Fig. 4. Aerial View of Point Beach Nuclear Power Plant. ANL Neg. No. 190-499. 


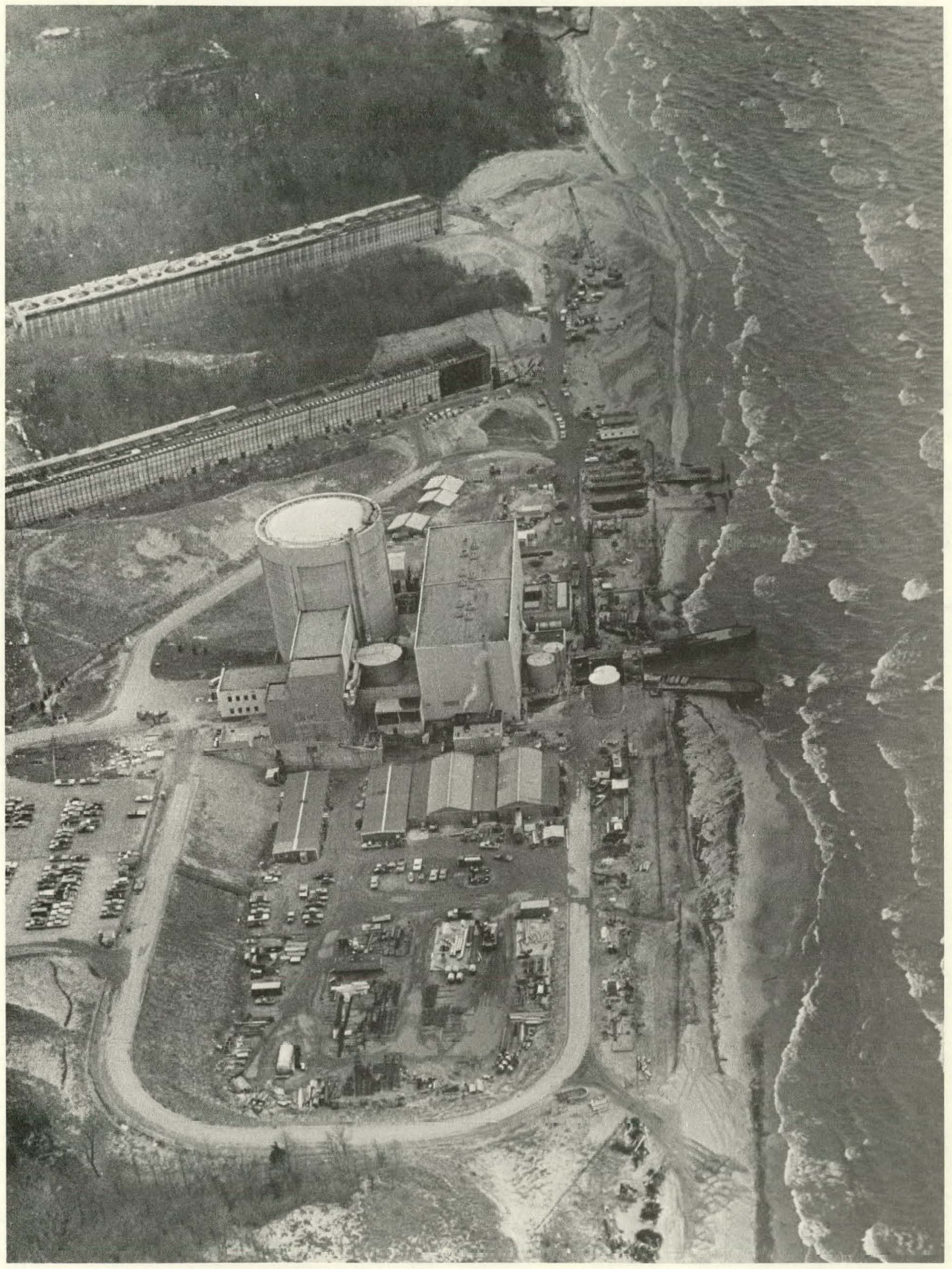

Fig. 5. Aerial View of Palisades Nuclear Power Plant 


\section{RESULTS OF FIELD MEASUREMENTS}

During the 1972 field year, seven jet-regime studies were conducted at the two power plants described in Sec. III. Specifically, these studies were at the Point Beach Nuclear Power Plant Unit 1 on May 18, May 23, July 13, and September 9, 1972, and at the Palisades Nuclear Power Plant on June 14, July 19, and October 10, 1972. In addition, one survey was conducted late in the 1971 field year (November 3, 1971) as a preliminary feasibility study of the technique (see Appendix B). Data collected include measurements of velocity and temperature in the near-field region of the thermal plume at the $0.5-, 1.0-, 1.5-, 2.0-, 2.5-$, and 3.0-m depths, along with measurements of ambient lake and meteorological conditions. Bottom depth was also measured at various locations. From the bottom-depth data, approximate depth contours were drawn near the outfalls and are shown in Figs, 6 and 7 . The points indicate positions at which data were taken.* Results of the jet-regime measure ments are shown in Figs. 8-48 for the dates indicated. The figures show station locations at which jet velocities and temperatures were measured. The velocity is represented vectorially at each station location. In addition, the current speed, current direction, and temperature at each station are listed in a table on each figure. Current direction is given in degrees as measured from magnetic north. Also listed on the figures are the ambient lake and meteorological data, as well as the plant operating data. Temperature and velocity centerlines and widths are shown in most cases. The widths represent the lateral distance from the centerline at which the appropriate parameter has reached a value halfway between the centerline value and the ambient value. The mathematical fitting technique used to obtain the centerlines and widths is described in Sec.V. Centerlines and widths are not shown for any depths for the jet-regime studies conducted at the Palisades Plant on June 14 and July 19, 1972. Typically, the Palisades outfall produces a very wide jet, and the data on these dates did not lend themselves to the type of analysis necessary for determining centerlines and widths.

*Dotted lines indicate estimated contours for which no data were available. 

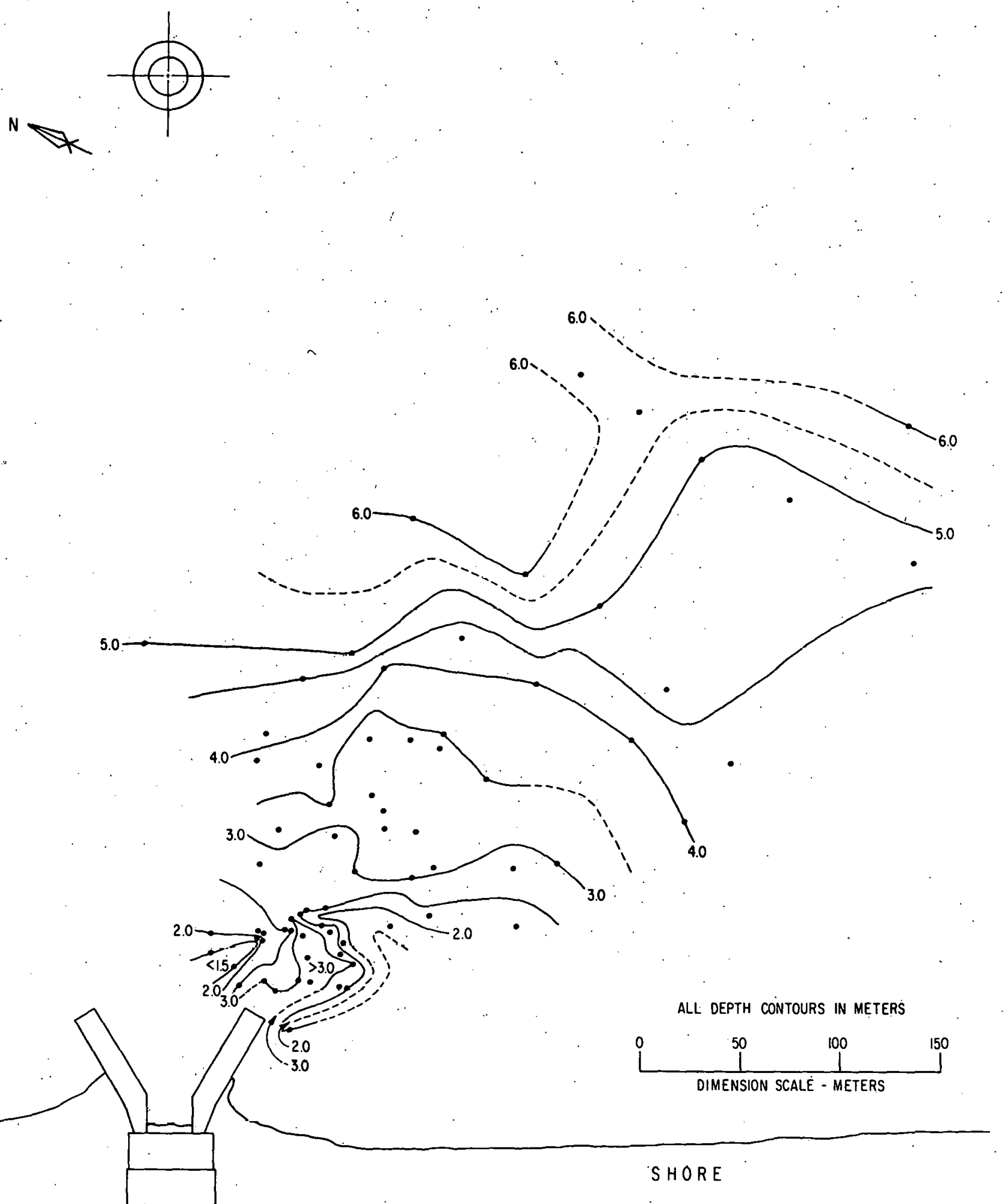

Fig. 6. A pproximate Depth Contours near Point Beach Outfall. ANL Neg. No. 190-878. 


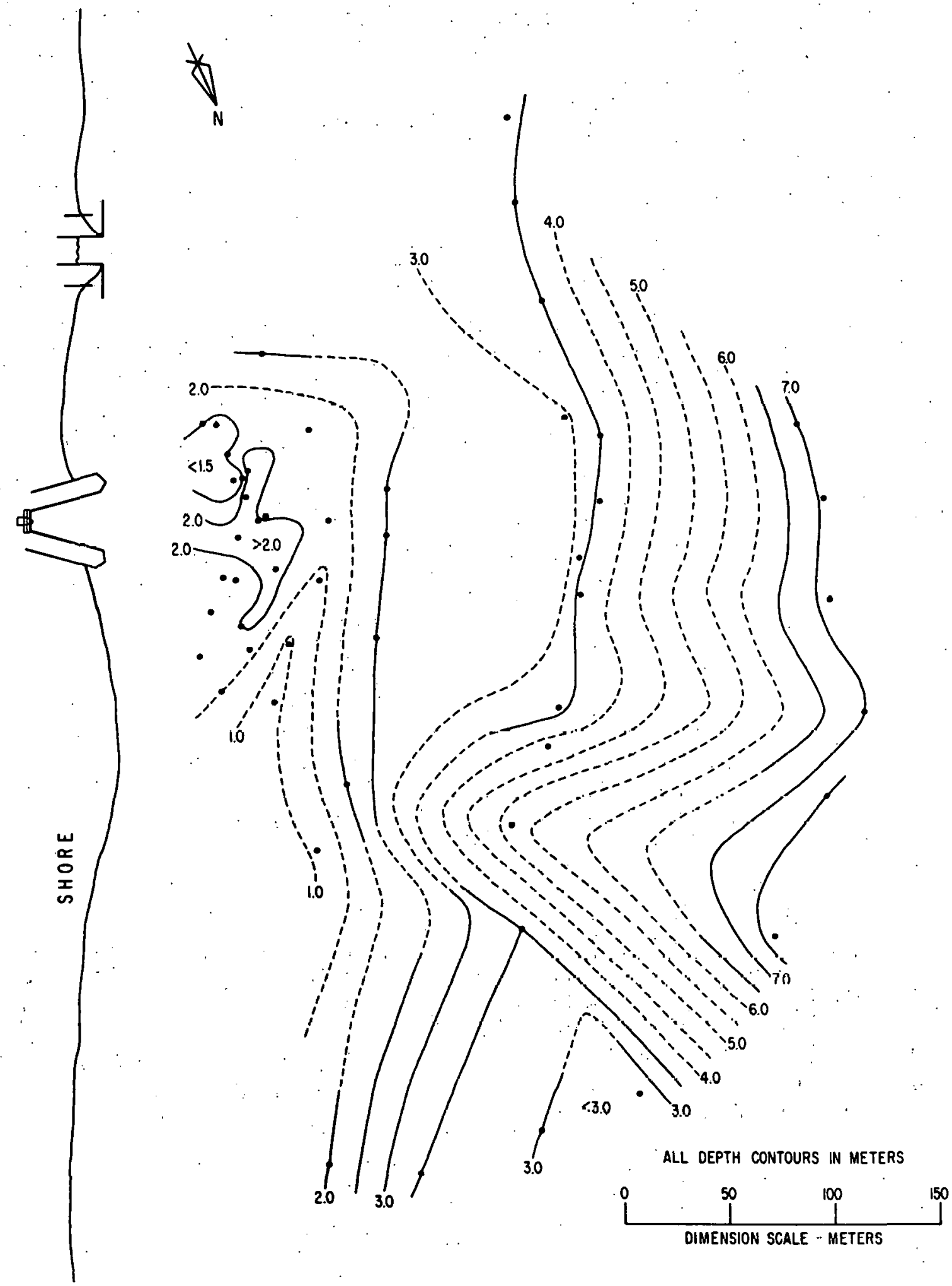

Fig. 7. Approximate Depth Contours near Palisades Outfall. ANL Neg. No. 190-880. 


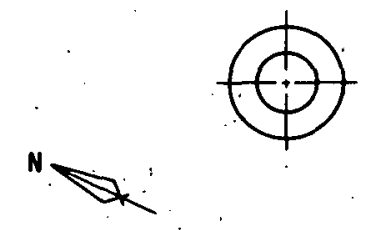

\begin{tabular}{|c|c|c|c|}
\hline $\begin{array}{l}\text { STATIOH } \\
\text { NUMBER. }\end{array}$ & $\begin{array}{c}\text { CURRENT } \\
\text { SPEED } \\
\text { (cm/sec) }\end{array}$ & $\begin{array}{c}\text { CURRENT } \\
\text { DIRECTION } \\
\left({ }^{\circ}\right)\end{array}$ & $\begin{array}{c}\text { TEMPERATURE } \\
\left({ }^{\circ} \mathrm{C}\right)\end{array}$ \\
\hline 1 & 0 & - & 11.0 \\
\hline 2 & 0 & $=$ & 11.2 \\
\hline 3 & \multicolumn{3}{|c|}{ NOT MEASURED } \\
\hline 4 & 21.1 & 115 & 14.0 \\
\hline 5 & 26.1 & 80 & 14.5 \\
\hline 6 & 16.7 & 105 & 11.1 \\
\hline 7 & 0 & - & 12.7 \\
\hline 8 & $\theta$ & - & 13.3 \\
\hline 9 & 40.5 & 125 & 14.5 \\
\hline 10 & 44.5 & 120 & $13.0^{\circ}$ \\
\hline 11 & 0 & - & 10.5 \\
\hline 12 & 0 & - & 10.6 \\
\hline 13 & 9.5 & .95 & 11.0 \\
\hline 14 & 58.4 & 105 & 16.7 \\
\hline 15 & 36.1 & 125 & 16.0 \\
\hline 16 & 27.8 & 140 & 16.0 \\
\hline 17 & .128 & 145 & 13.8 \\
\hline 18 & 9.5 & 125 & 13.4 \\
\hline 19 & 67.8 & 95 & 17.7 \\
\hline
\end{tabular}

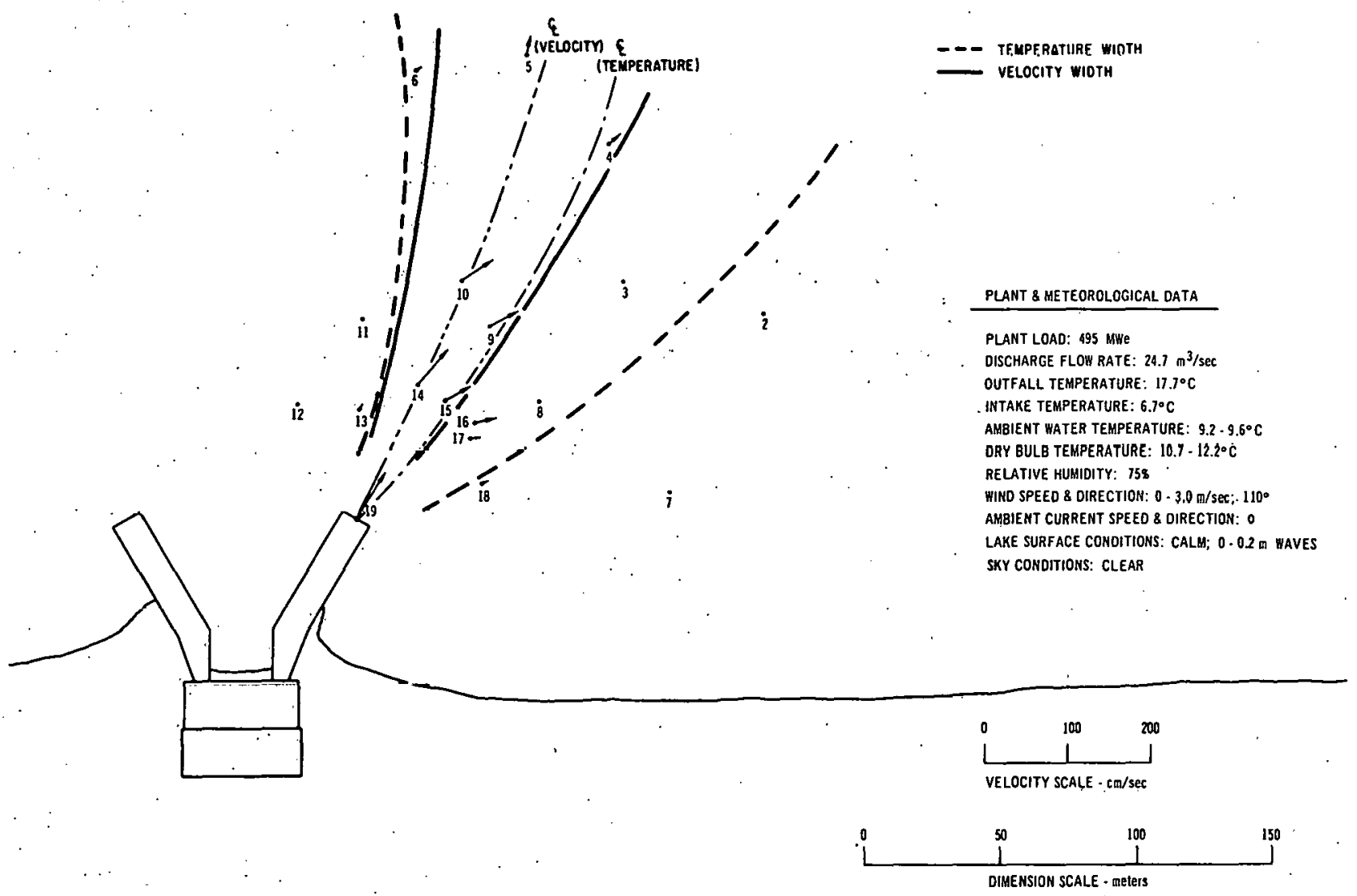

Fig. 8. Jet-regime Study for 0.5-m Depth at Point Beach Power Plant (Jnit 1): May 18, 1972, 1115-1440. Hours. ANL Neg. No. 190-761 Rev. 1. 


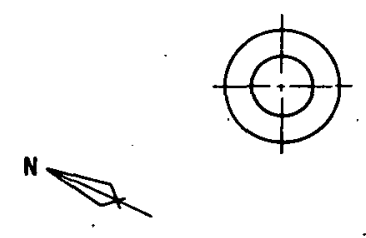

\begin{tabular}{|c|c|c|c|}
\hline $\begin{array}{l}\text { STATION } \\
\text { NUMBER }\end{array}$ & $\begin{array}{l}\text { CURRENT } \\
\text { SPEED } \\
(c / \mathrm{sec})\end{array}$ & $\begin{array}{l}\text { CURRENT } \\
\text { OHRECTION } \\
\left(0^{\circ}\right)\end{array}$ & $\begin{array}{l}\text { TEMPERATURE } \\
\left({ }^{\circ} \mathrm{C}\right)\end{array}$ \\
\hline$I$ & 0 & $=$ & 9.7 \\
\hline 2 & 0 & $=$ & 11.0 \\
\hline 3 & 6.1 & 145 & 12.6 \\
\hline 4 & 16.7 & 140 & 14.0 \\
\hline 5 & 22.2 & 85 & 13.5 \\
\hline 6 & 9.5 & 110 & 10.6 \\
\hline 7 & \multicolumn{3}{|c|}{ LAKE BOTTOM } \\
\hline 8 & 0 & - & 13.1 \\
\hline 9 & 36.1 & 110 & 14.4 \\
\hline 10 & 41.7 & 110 & 13.9 \\
\hline 11 & 0 & - & 9.1 \\
\hline 12 & 0 & $=$ & 8.5 \\
\hline 13 & 8.3 & 110 & 10.3 \\
\hline 14 & 47.3 & 95 & 16.0 \\
\hline 15 & 30.6 & 100 & 14.5 \\
\hline 16 & 22.2 & 115 & 16.7 \\
\hline 11 & 12.2 & 135 & 13.6 \\
\hline 18 & 0 & - & 131 \\
\hline 19 & .65 .2 & 25. & 17.7 \\
\hline
\end{tabular}

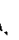

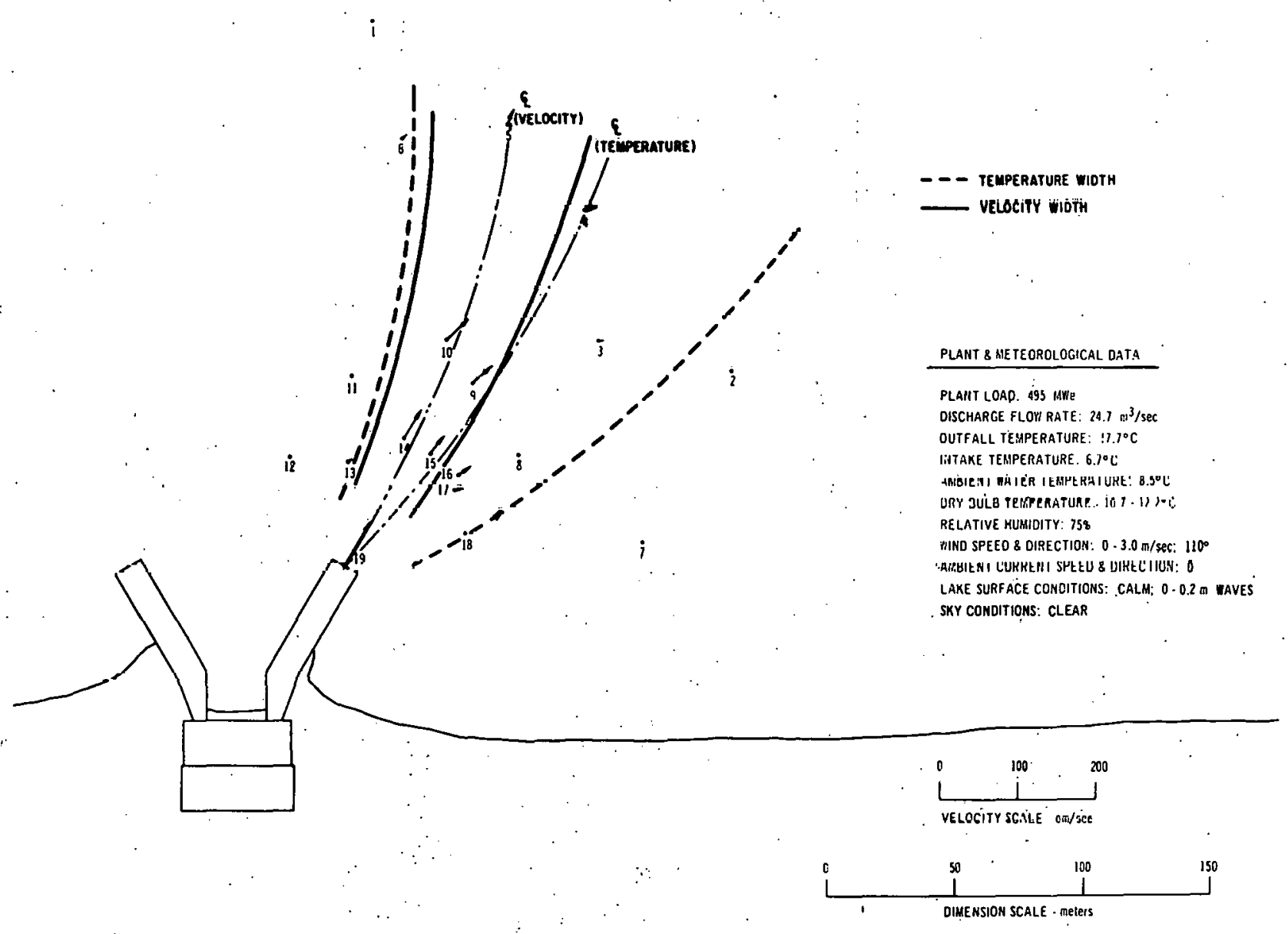

Fig. 9. Jet-regime Study for 1.0-m Depth at Point Beach Power Plant (Unit 1):

May 18, 1972, 1115-1440 Hours. ' ANL Neg. No. 190-895. 

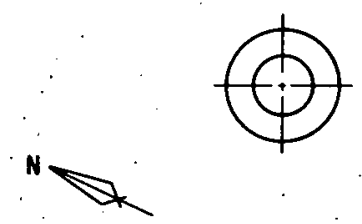

\begin{tabular}{|c|c|c|c|}
\hline $\begin{array}{l}\text { STATION } \\
\text { NUMBER }\end{array}$ & $\begin{array}{l}\text { CURRENT } \\
\text { SPEED } \\
(\mathrm{cm} / \mathrm{sec}) .\end{array}$ & $\begin{array}{c}\text { CURRENT } \\
\text { OIRECTION } \\
\left({ }^{\circ}\right) \\
\end{array}$ & $\begin{array}{c}\text { TEMPERATURE } \\
\left({ }^{\circ} \mathrm{C}\right)\end{array}$ \\
\hline 1 & \multicolumn{3}{|c|}{ NOT MEASURED } \\
\hline 2 & 8.9 & 10 & 10.4 \\
\hline 3 & \multicolumn{3}{|c|}{ NOT MEASURED } \\
\hline 4 & 10.6 & 135 & 13.2 \\
\hline 5 & 23.9 & 85 & 12.6 \\
\hline 6 & 0 & - & 9.7 \\
\hline 1 & \multicolumn{3}{|c|}{ LAKE BOTTOM } \\
\hline 8 & 0 & - & 12.1 \\
\hline 9 & 33.4 & 115. & 15.0 \\
\hline 10 & 29.5 & 1000 & 13.4 \\
\hline II & \multicolumn{3}{|c|}{ NOT MEASURED } \\
\hline 12 & 0 & - & 8.3 \\
\hline 13 & 8.3 & 170 & 8.4 \\
\hline 14 & 50.0 & 105 & 16.2 \\
\hline 15 & 22.2 & 105 & 15.0 \\
\hline 16 & 18.3 & 125 & 14.4 \\
\hline 11 & 5.6 & 115 & 13.2 \\
\hline 18 & 0 & - & 12.5 \\
\hline 19 & 62.3 & 95 & 17.7 \\
\hline
\end{tabular}

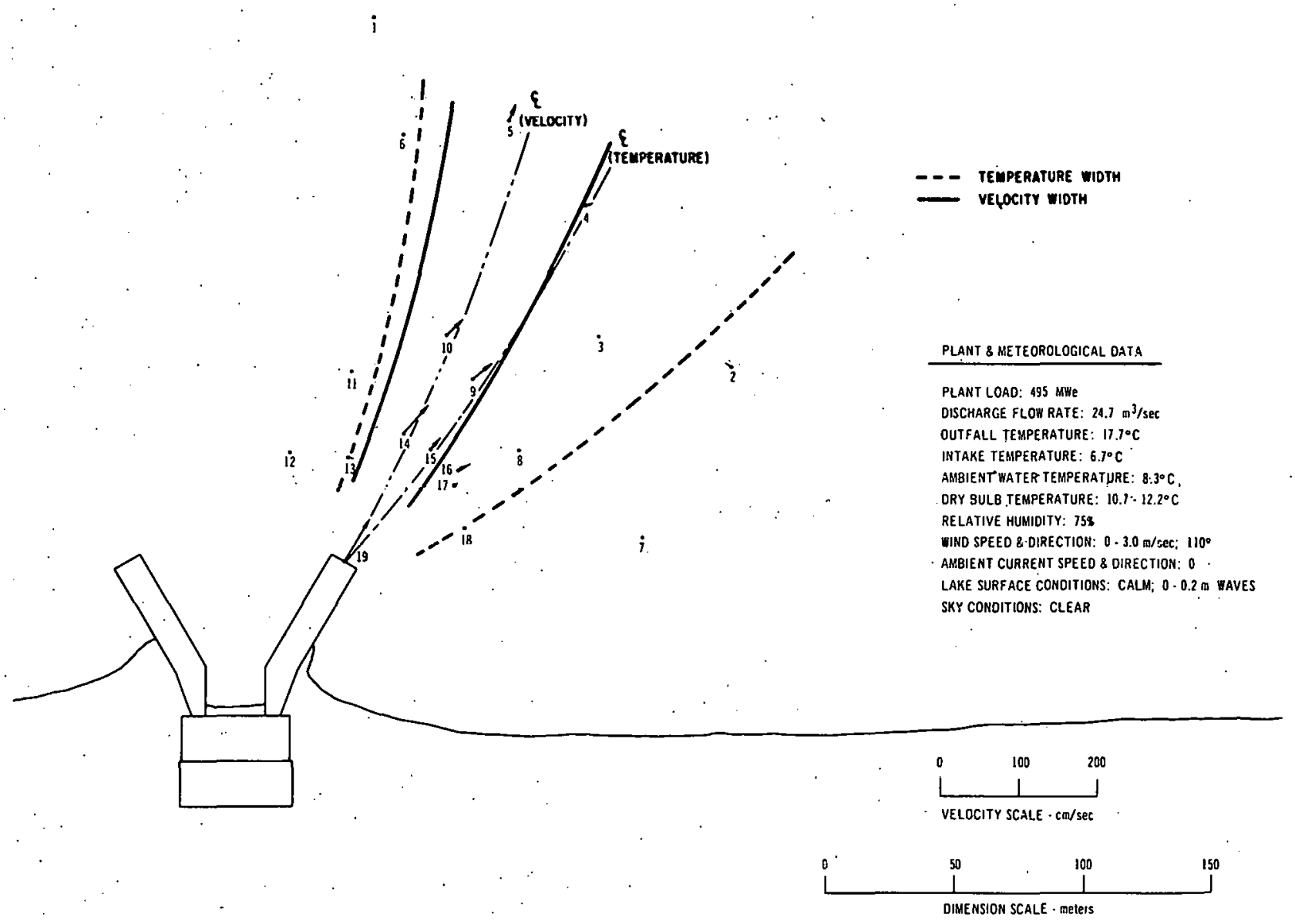

Fig. 10. Jet-regime Study for $1.5-m$ Depth at Point Bcach Power Plant (Unit 1): May 18, 1972, 1115-1440 Hours. ANL Neg. No. 190-893. 


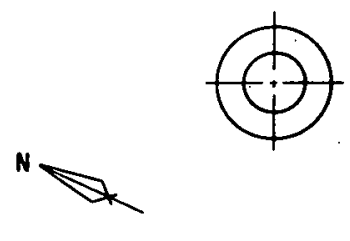

\begin{tabular}{|c|c|c|c|}
\hline $\begin{array}{l}\text { SI I TIVN } \\
\text { SULKBEER }\end{array}$ & $\begin{array}{l}\text { CURRENT } \\
\text { SPEEO } \\
1 \mathrm{Ca} / \mathrm{sec}\end{array}$ & $\begin{array}{l}\text { CURPEENT } \\
\text { D1RTCCT10N } \\
\text { io: }\end{array}$ & $\begin{array}{l}\text { IEH:PERATURE } \\
1{ }^{\circ} \mathrm{C} \text { I }\end{array}$ \\
\hline 1. & 0 & - & 9.9 \\
\hline 2 & 8.3 & 15 & 8.3 \\
\hline 2 & 0 & - & 10.8 \\
\hline 4 & 6.7 & 135 & 10.4 \\
\hline 5 & 19.5 & 80 & 12.5 \\
\hline 6 & 0 & - & 9.2 \\
\hline 7 & \multicolumn{3}{|c|}{ LAKE BOTIOM } \\
\hline 8 & \multicolumn{3}{|c|}{$\angle A K E$ BOTTOM } \\
\hline 9 & 21.7 & 90 & 14.5 \\
\hline 10 & 25.0 & 125 & 11.5 \\
\hline II & 0 & - & 8.3 \\
\hline 12 & \multirow{2}{*}{\multicolumn{3}{|c|}{ LAKE $B O T T O M$}} \\
\hline 13 & & & \\
\hline 11 & 500 & 105 & 15.0 \\
\hline 15 & 27.8. & 110 & 14.2 \\
\hline 16 & 10.0 & 110 & 13.3 \\
\hline 17 & 0 & - & 12.4 \\
\hline 18 & 0 & - & 11.9 \\
\hline 19 & 8.3 & 99 & ii.. \\
\hline
\end{tabular}

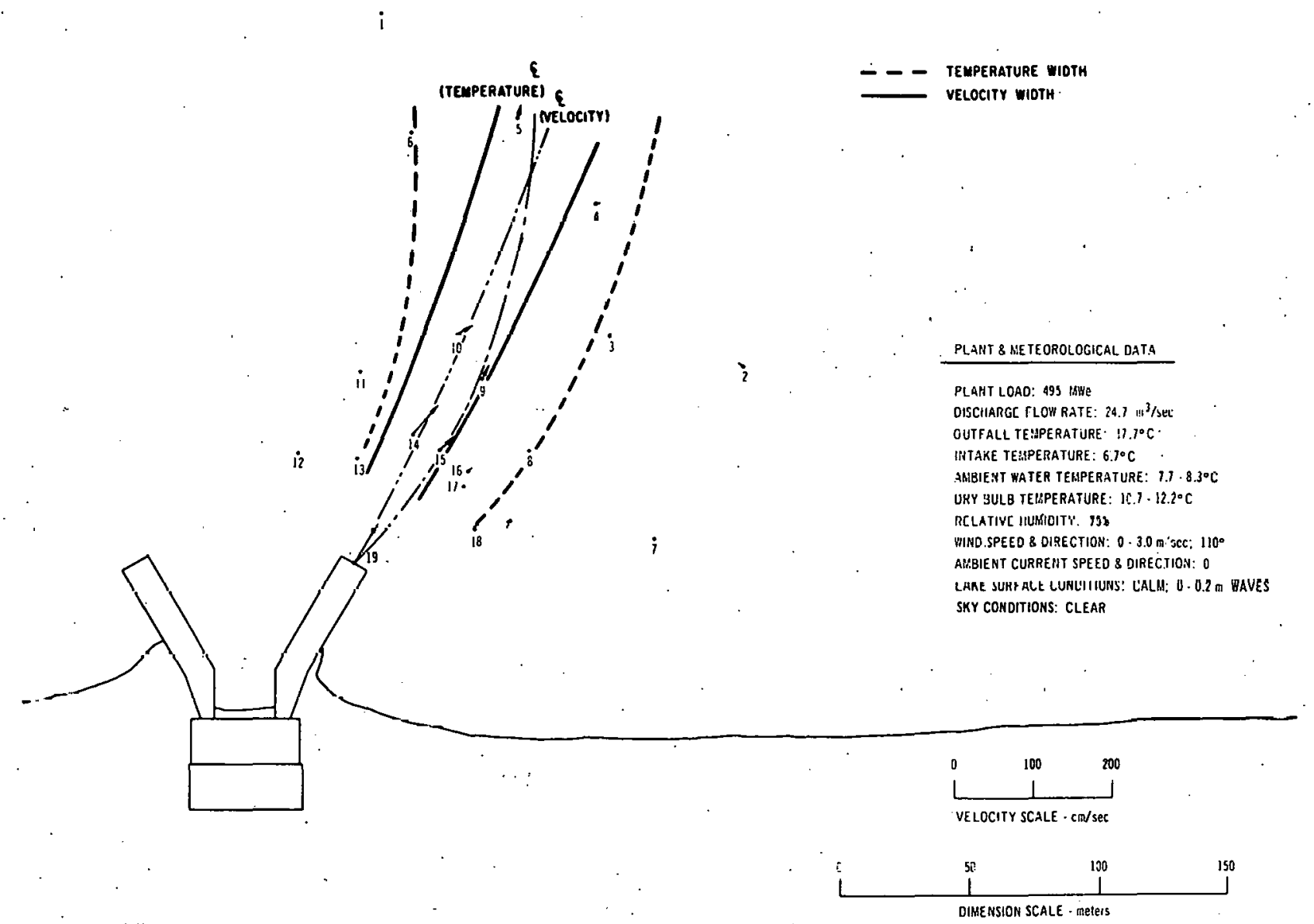

Fig. 11. Jet-regime Study for 2.0-m Depth at Point Beach Power Plant (Unit 1): May 18, 1972, 1115-1440 Hours. ANL Neg. No. 190-899. 

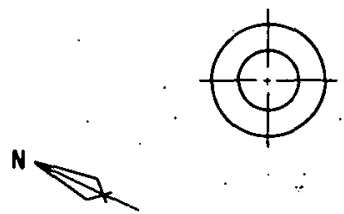

\begin{tabular}{|c|c|c|c|}
\hline $\begin{array}{l}\text { STATION } \\
\text { NUMBER }\end{array}$ & $\begin{array}{l}\text { CURRENT } \\
\text { SPEEDO } \\
\text { (etm/sec) }\end{array}$ & $\begin{array}{l}\text { CURRENT } \\
\text { OIRECTION } \\
\left({ }^{\circ}\right)\end{array}$ & $\begin{array}{l}\text { TEMPERATURE } \\
\left({ }^{\circ} \mathrm{C}\right)\end{array}$ \\
\hline 1 & \multicolumn{3}{|c|}{ NOT MEASURED } \\
\hline 2 & 7.8 & 35 & 7.8 \\
\hline 3 & 0 & - & 9.0 \\
\hline 4 & 0 & - & 12.0 \\
\hline 5 & 15.6 & 80 & 11.1 \\
\hline 6 & 0 & - & 8.8 \\
\hline 1 & \multirow{2}{*}{\multicolumn{3}{|c|}{$\begin{array}{l}\text { LAKE BOTTOM } \\
\text { LAKE BOTTOM }\end{array}$}} \\
\hline 8 & & & TOM \\
\hline 9 & 16.7 & 95 & 13.2 \\
\hline 10 & 17.8 & 130 & 10.0 \\
\hline$\pi$ & \multicolumn{3}{|c|}{ LAKE BOTTOM } \\
\hline 12 & \multicolumn{3}{|c|}{ LAKE BOTTOM } \\
\hline 13 & \multirow{2}{*}{\multicolumn{3}{|c|}{ LAKE BOTTOM }} \\
\hline 14 & \multirow{2}{*}{\multicolumn{3}{|c|}{$\begin{array}{ll}\text { LAKE } & \text { BOTTOM } \\
\text { LAKE } & \text { BOTTOM }\end{array}$}} \\
\hline 15 & & & \\
\hline 16 & \multicolumn{3}{|c|}{ LAKE BOTTOM } \\
\hline 17 & 6.7 & 45 & 12.8 \\
\hline 18 & \multicolumn{3}{|c|}{ LAKE BOTTOM } \\
\hline 19 & 55.6 & 25 & 117. \\
\hline
\end{tabular}

i

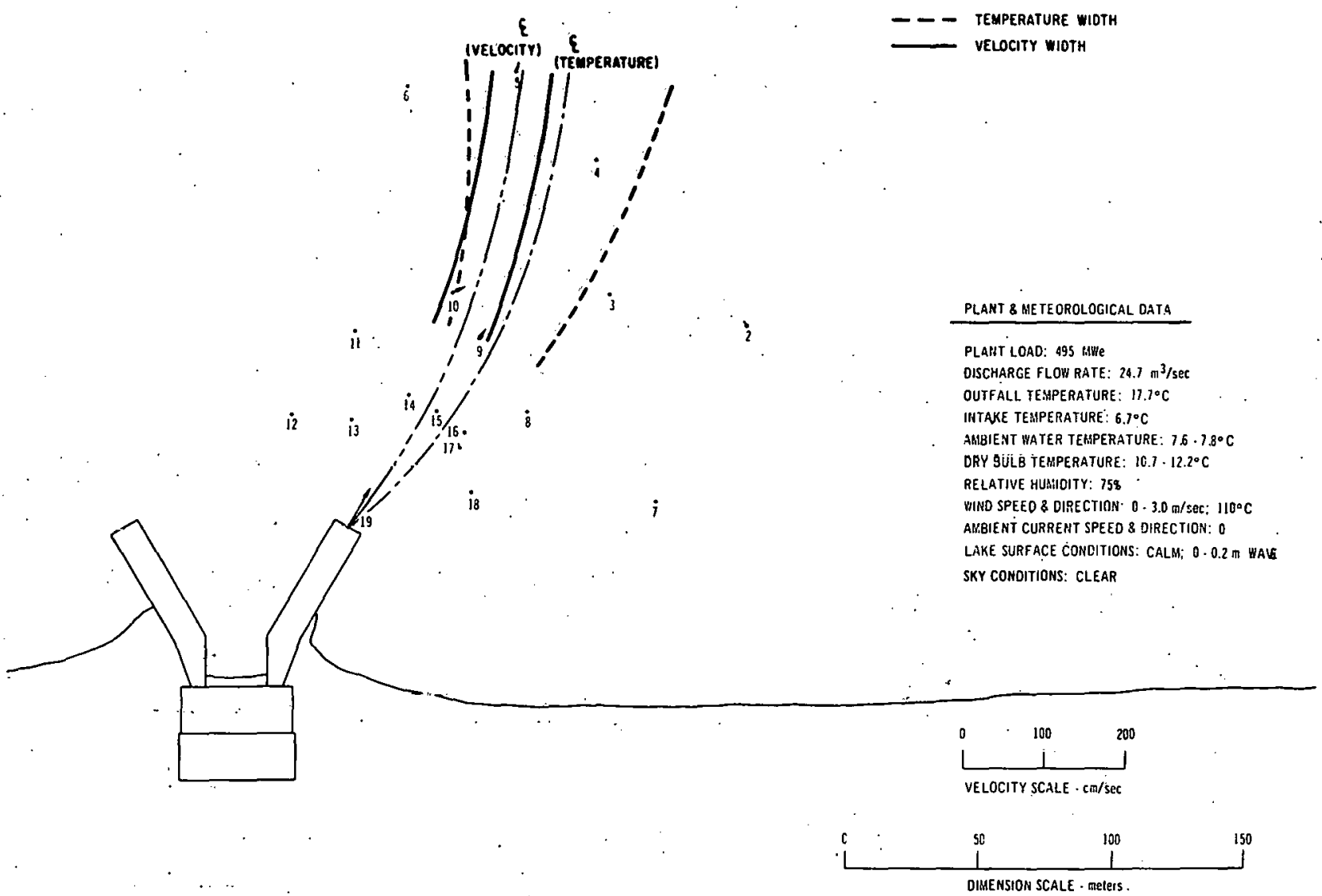

Fig. 12. Jet-regime Study for 2.5-m Depth at Pnint Beach Power Plant (Unit 1): May. 18, 1972, 1115-1440 Hours. ANL Neg. No. 190-898. 


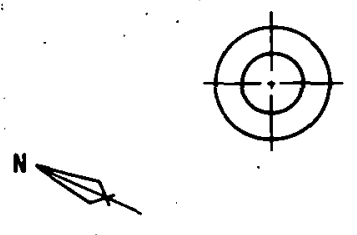

\begin{tabular}{|c|c|c|c|}
\hline $\begin{array}{l}\text { STATION } \\
\text { NUMBER }\end{array}$ & $\begin{array}{l}\text { CURRENT } \\
\text { SPEED } \\
\text { (cm/sec) }\end{array}$ & $\begin{array}{c}\text { CURRENT } \\
\text { DIRECJION } \\
\left(1^{\circ}\right)\end{array}$ & $\begin{array}{l}\text { TEMPERATURE } \\
\left({ }^{\circ} \mathrm{C}\right)\end{array}$ \\
\hline 1 & 0 & - & 8.0 \\
\hline 2 & \multicolumn{3}{|c|}{ LAKE BOTTOM } \\
\hline 3 & \multicolumn{3}{|c|}{ MOT MEASURED } \\
\hline 4 & \multicolumn{3}{|c|}{ NOT MEASURED } \\
\hline 5 & \multicolumn{3}{|c|}{ NOT MEASURED } \\
\hline 6 & \multicolumn{3}{|c|}{ NOT MEASURED } \\
\hline 7 & \multicolumn{3}{|c|}{ LAKE BOTTOM } \\
\hline 8 & \multicolumn{3}{|c|}{ LAKE BOTTOM } \\
\hline 9 & \multicolumn{3}{|c|}{ LAKE BOTTOM } \\
\hline 10 & \multicolumn{3}{|c|}{ LAKE BOTTOM } \\
\hline 11 & \multicolumn{3}{|c|}{ LAKE BOTTOM } \\
\hline 12 & \multicolumn{3}{|c|}{ LAKE BOTTOM } \\
\hline I] & \multicolumn{3}{|c|}{ LAKE BOTTOM } \\
\hline 14 & \multicolumn{3}{|c|}{ LAKE BOTTON } \\
\hline 15 & \multicolumn{3}{|c|}{ LAKE BOTTOM } \\
\hline 16 & \multicolumn{3}{|c|}{ LAKE BOTTOM } \\
\hline 17 & \multicolumn{3}{|c|}{ LAKE. OOTTOM } \\
\hline 18 & \multicolumn{3}{|c|}{ LAKE BOTTON } \\
\hline is & 91.8 & 95 & 17.7 \\
\hline
\end{tabular}

i $\quad$;

$i$

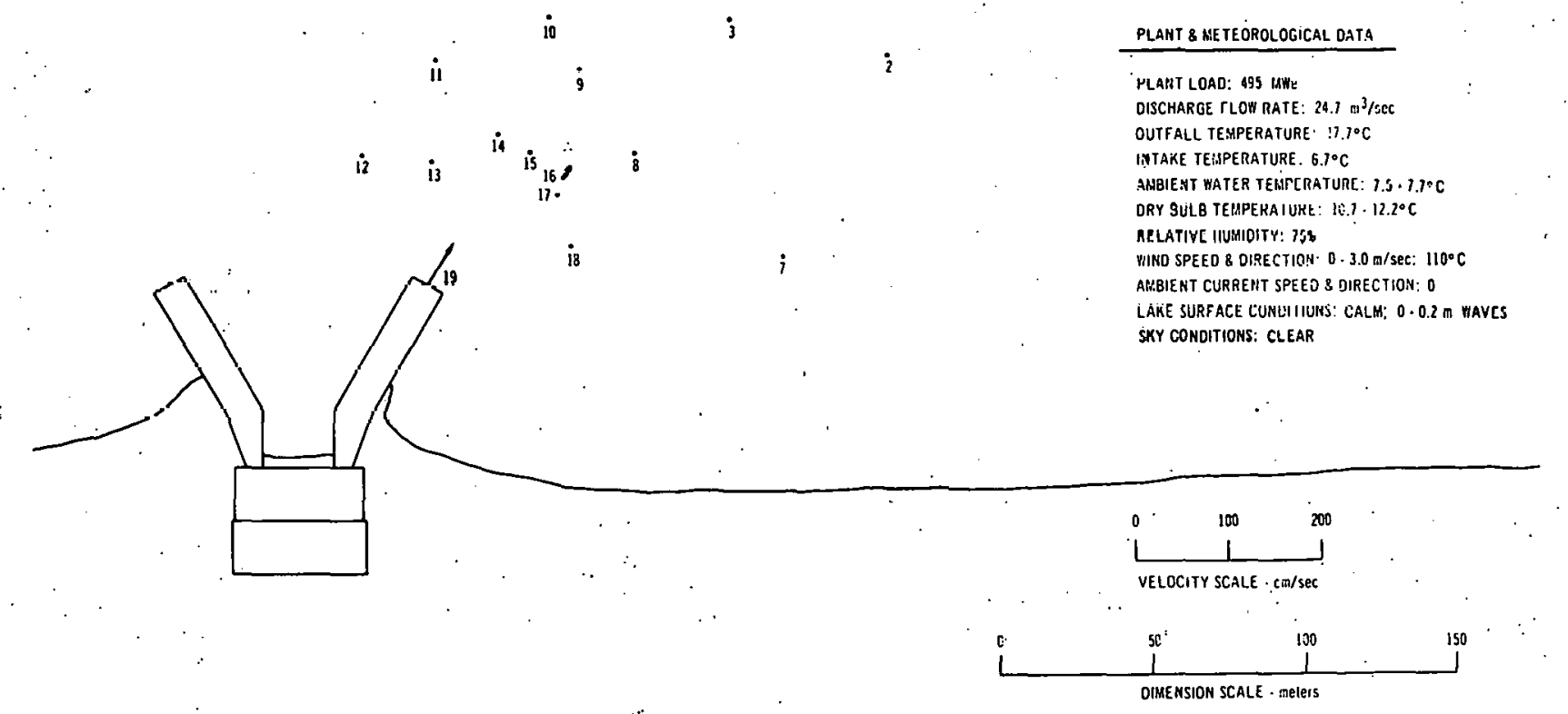

Fig. 13. Jet-regime Study for 3.0-m Depth at Point Beach Power Plant (Unit 1):

May 18, 1972, 1115-1440 Hours. ANL Neg. No. 190-889. 


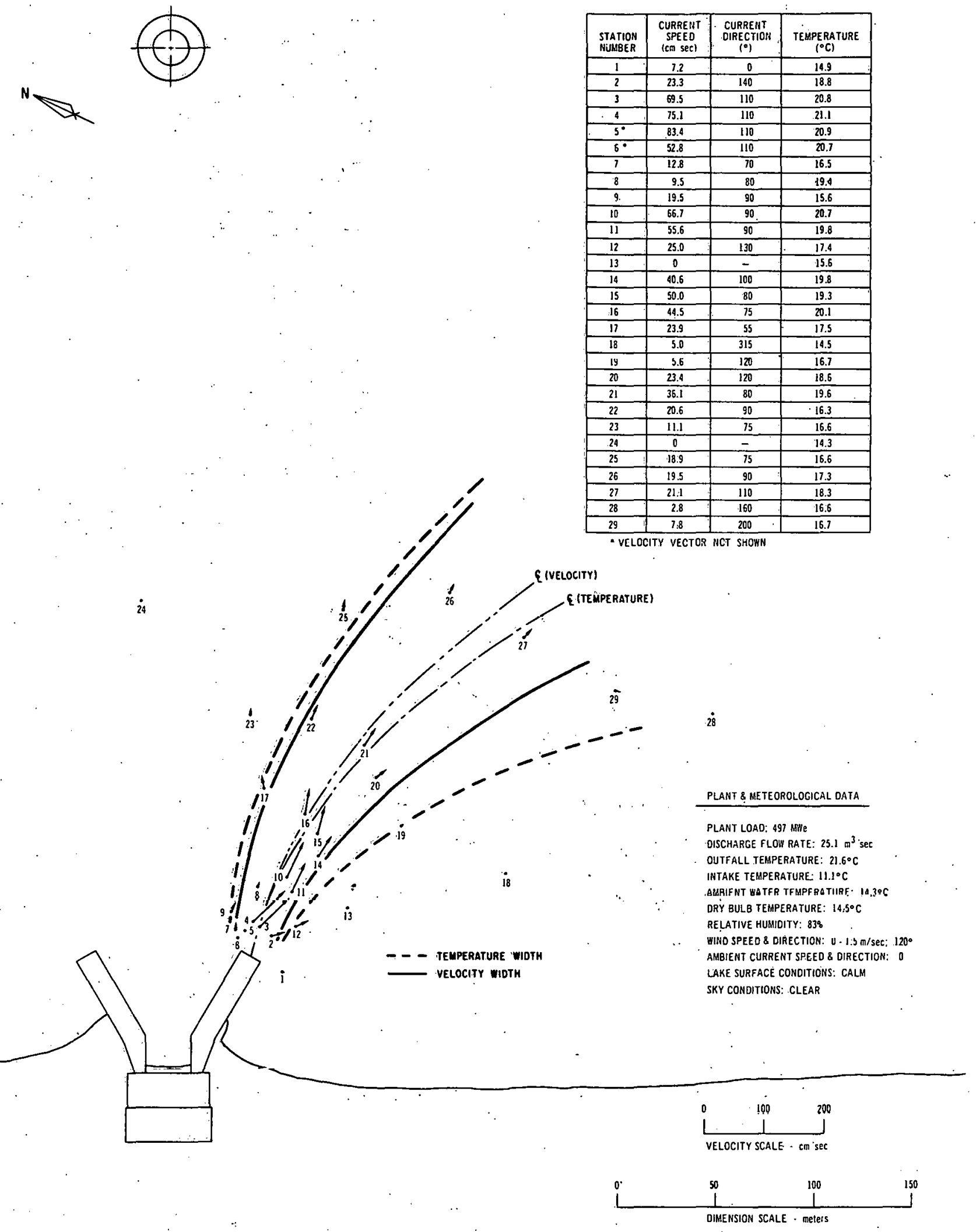

Fig. 14. Jet-reginte Study for 0.5-m Depth at Point Beach Power Plant (Unit 1): May 23, 1972, 0945-1700 Hours. ANL Neg. No. 190-760 Rev. 1. 

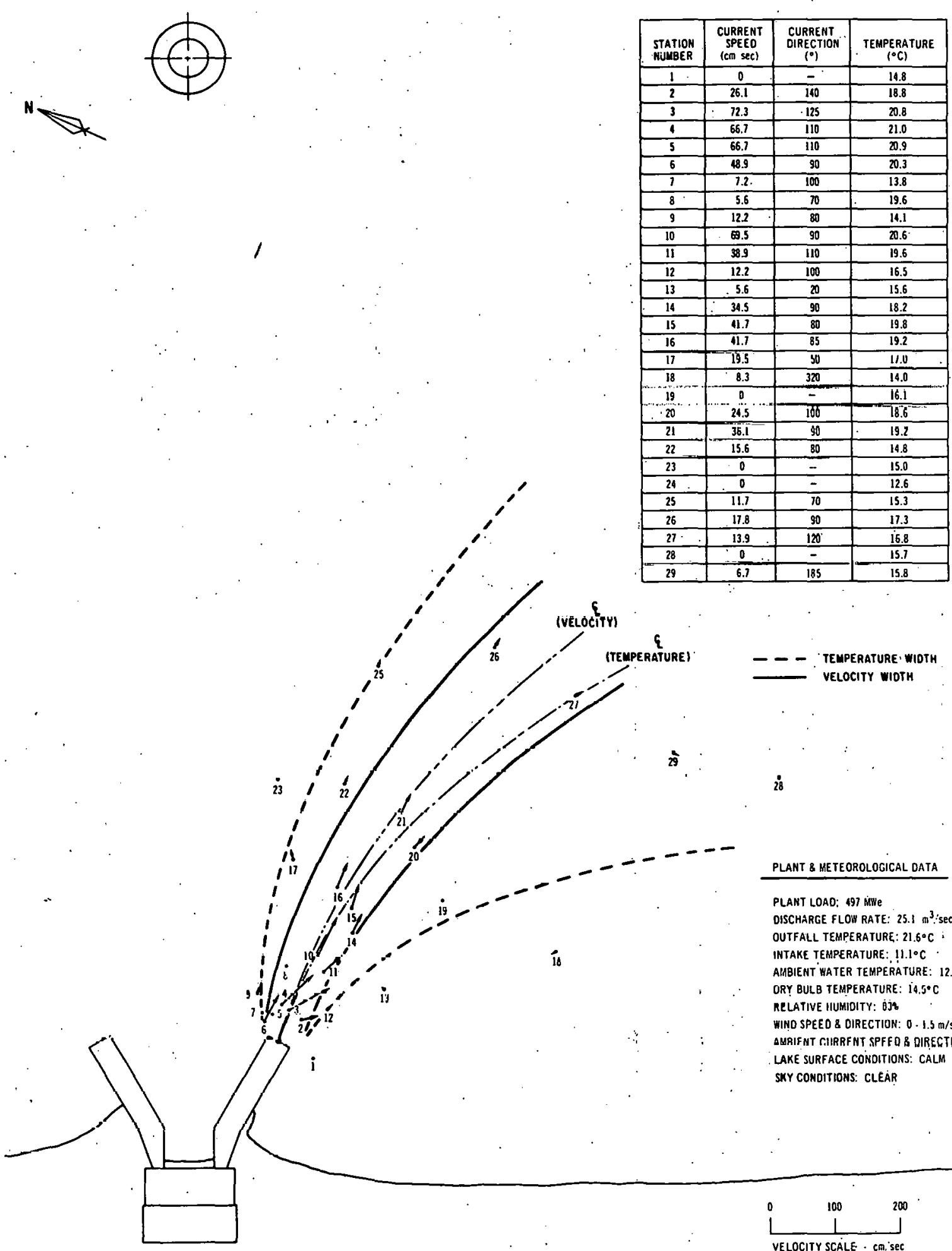

\begin{tabular}{|c|c|c|c|}
\hline $\begin{array}{l}\text { STATION } \\
\text { NuMGER }\end{array}$ & $\begin{array}{c}\text { CURRENT } \\
\text { SPEEO } \\
\text { (em sec) }\end{array}$ & $\begin{array}{l}\text { CURRENT } \\
\text { OIRECTION } \\
\left({ }^{\circ}\right)\end{array}$ & $\begin{array}{l}\text { TEMPERATURE } \\
\left({ }^{\circ} \mathrm{C}\right)\end{array}$ \\
\hline 1 & 0 & - & 14.8 \\
\hline 2 & 26.1 & 140 & 18.8 \\
\hline 3 & 72.3 & .125 & 20.8 \\
\hline 7 & 66.7 & 110 & 21.0 \\
\hline 5 & 66.7 & 110 & 20.9 \\
\hline 6 & 48.9 & 90 & 20.3 \\
\hline 7 & 7.2 . & 100 & 13.8 \\
\hline 8 & 5.6 & 70 & 19.6 \\
\hline 9 & 12.2 & 80 & 10.1 \\
\hline 10 & 由.5 & 90 & $20.6^{\circ}$ \\
\hline II & 38.9 & 110 & 19.6 \\
\hline 12 & 12.2 & 100 & 16.5 \\
\hline 13 & 5.6 & $x$ & 15.6 \\
\hline 14 & 34.5 & 90 & 18.2 \\
\hline 15 & 41.7 & 80 & 19.8 \\
\hline 16 & 4.7 & 85 & 19.2 \\
\hline 17 & 19.5 & 30 & 11.0 \\
\hline 18 & 8.3 & 320 & 14.0 \\
\hline 19 & 0 & $=$ & 16.1 \\
\hline 20 & $24.5^{\circ}$ & 106 & 786 \\
\hline 21 & 36.1 & 40 & 19.2 \\
\hline 22 & 15.6 & 80 & 14.8 \\
\hline 23 & 0 & - & 15.0 \\
\hline 24 & 0 & - & 12.6 \\
\hline 25 & 11.7 & 70 & 15.3 \\
\hline 26 & 177.8 & 30 & 17.3 \\
\hline 27 & 13.9 & $120^{\circ}$ & 16.8 \\
\hline 28 & 0 & - & 15.7 \\
\hline 29 & 6.7 & 185 & 15.8 \\
\hline
\end{tabular}

\section{(VELOCETYY)}

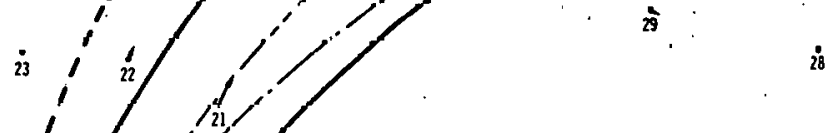

PLANT \& METEOROLOGICAL DATA

PLANT LOAO; 497 MNVIV

OISCHARGE FLOW RATE: $25.1 \mathrm{~m}^{3}$ 'sec

OUTFALL TEMPERATURE: $21.6^{\circ} \mathrm{C}$

INTAKE TEMPERATURE: $11.1^{\circ} \mathrm{C}$

AMBIENT WATER TEMPERATURE: $12.6^{\circ} \mathrm{C}$

ORY BULB TEMPERATURE: $14,5 \circ \mathrm{C}$

RELATIVE IIUMIOITY: OjJ

WIND SPEED \& OIRECTION: $0.1 .5 \mathrm{~m} / \mathrm{sec:} 120^{\circ}$

$\triangle M R I F N T$ CIIRRF NT SPFED \& DIRECTION; 0

LAKE SURFACE CONDITIONS: CALM

SKY CONDITIONS: CLEAR

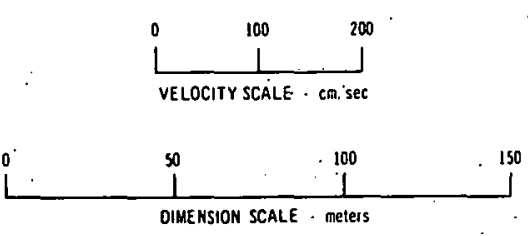

Fig. 15. Jet-regime Study for 1,0-m Depth at Point Beach Power Plant (Unit 1):

May 23, 1972, 0945-1700 Hours. ANL Neg. No. 190-891. 


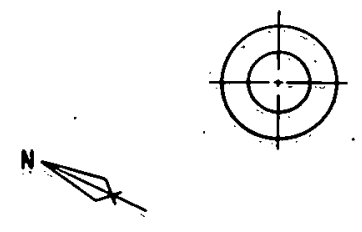

\begin{tabular}{|c|c|c|c|}
\hline $\begin{array}{l}\text { STATION } \\
\text { NUMBEER }\end{array}$ & $\begin{array}{c}\text { CURRENT } \\
\text { SPEED } \\
(\mathrm{Cm} \mathrm{sec})\end{array}$ & $\begin{array}{l}\text { CURRENT } \\
\text { CIRECIION } \\
\left\{{ }^{\circ} !\right.\end{array}$ & $\begin{array}{l}\text { TEMPERATURE } \\
\left({ }^{\circ} \mathrm{C}\right)\end{array}$ \\
\hline 1 & 0 & - & 14.7 \\
\hline 2 & 18.9 & 110 & 17.2 \\
\hline 3 & 77.8 & 135 & 20.8 \\
\hline 4 & 61.2 & 100 & 21.1 \\
\hline 5 & 61.2 & 100 & 21.0 \\
\hline 6 & 42.8 & 100 & 19.8 \\
\hline 7 & 9.5 & 150 & 12.7 \\
\hline 8 & 7.2 & 75 & 18.8 \\
\hline 9 & 9.5 & 220 & 14.3 \\
\hline 10 & 47.3 & 90 & 19.7 \\
\hline 11 & 23.9 & 70 & 17.8 \\
\hline 12. & 5.6 & 90 & 15.9 \\
\hline 13 & 10.0 & 350 & 14.7 \\
\hline 14 & 29.5 & 70 & 18.7 \\
\hline 15 & 50.0 & 95 & 18.6 \\
\hline 16 & 43.4 & 90 & 18.7 \\
\hline 17 & 5.6 & 75 & 14.9 \\
\hline 18 & 5.6 & 305 & 13.7 \\
\hline 19 & 0 & $\overline{-}$ & 15.0 \\
\hline 20 & 20.6 & 100 & 17.8 \\
\hline 21 & 30.0 & 90 & 18.8 \\
\hline 22 & 6.7 & 115 & 13.8 \\
\hline 23 & 0 & - & $13: 8$ \\
\hline 24 & 0 & - & 13.5 \\
\hline 25 & 9.5 & 80 & 14.7 \\
\hline 26 & 12.2 & 80 & 16.8 \\
\hline 27 & 9.5 & 120 & 15.8 \\
\hline 28 & 0 & - & 14.9 \\
\hline 29 & 0 & - & 15.1 \\
\hline
\end{tabular}

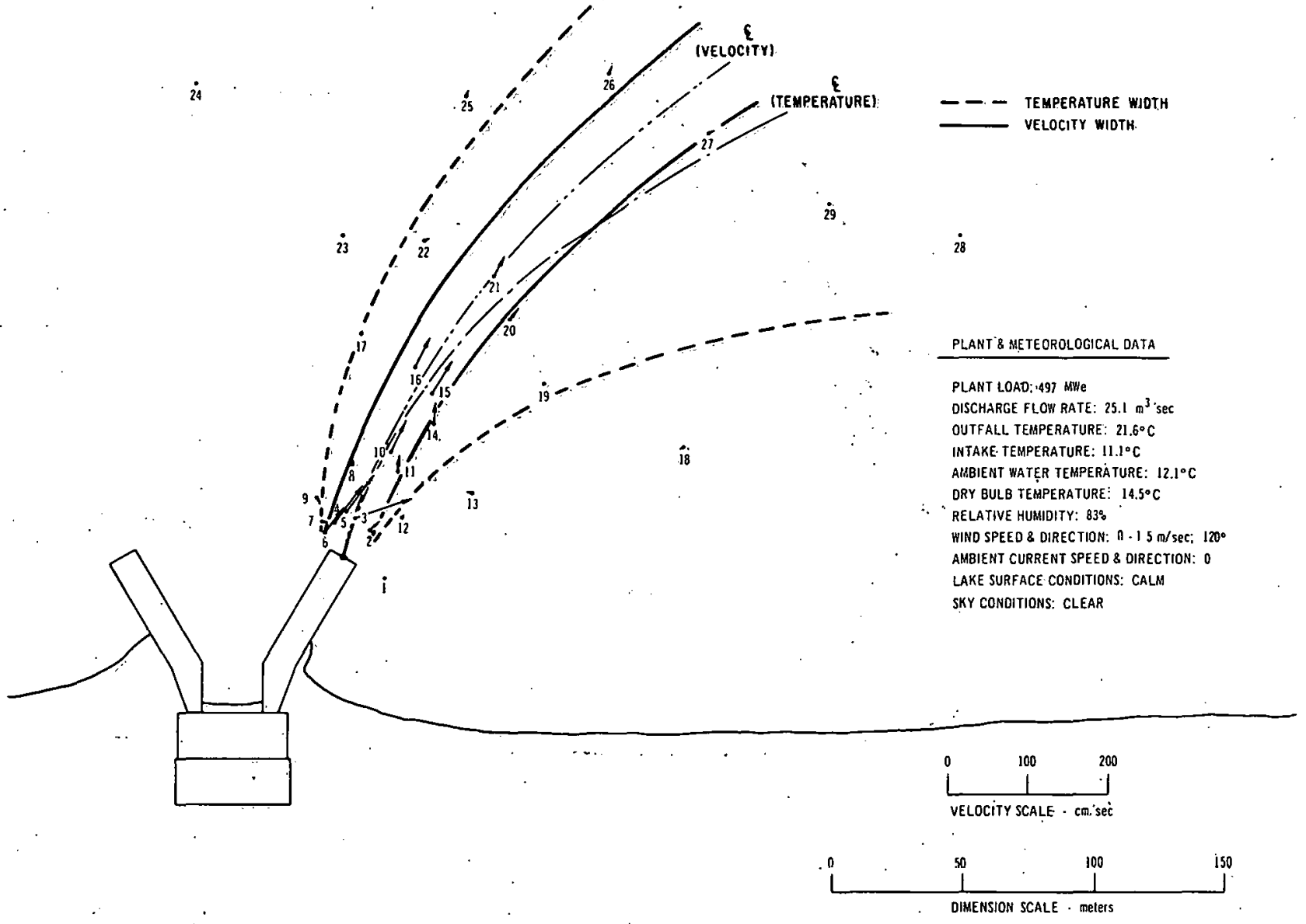

Fig. 16. Jct-rcgime Etudy for 1,6-m Depth at Point Bcach Powcr Plant (Unit 1): May 23, 1.972, 0945-1.700 Hours. ANt. Neg. Nò. 1.90-906. 


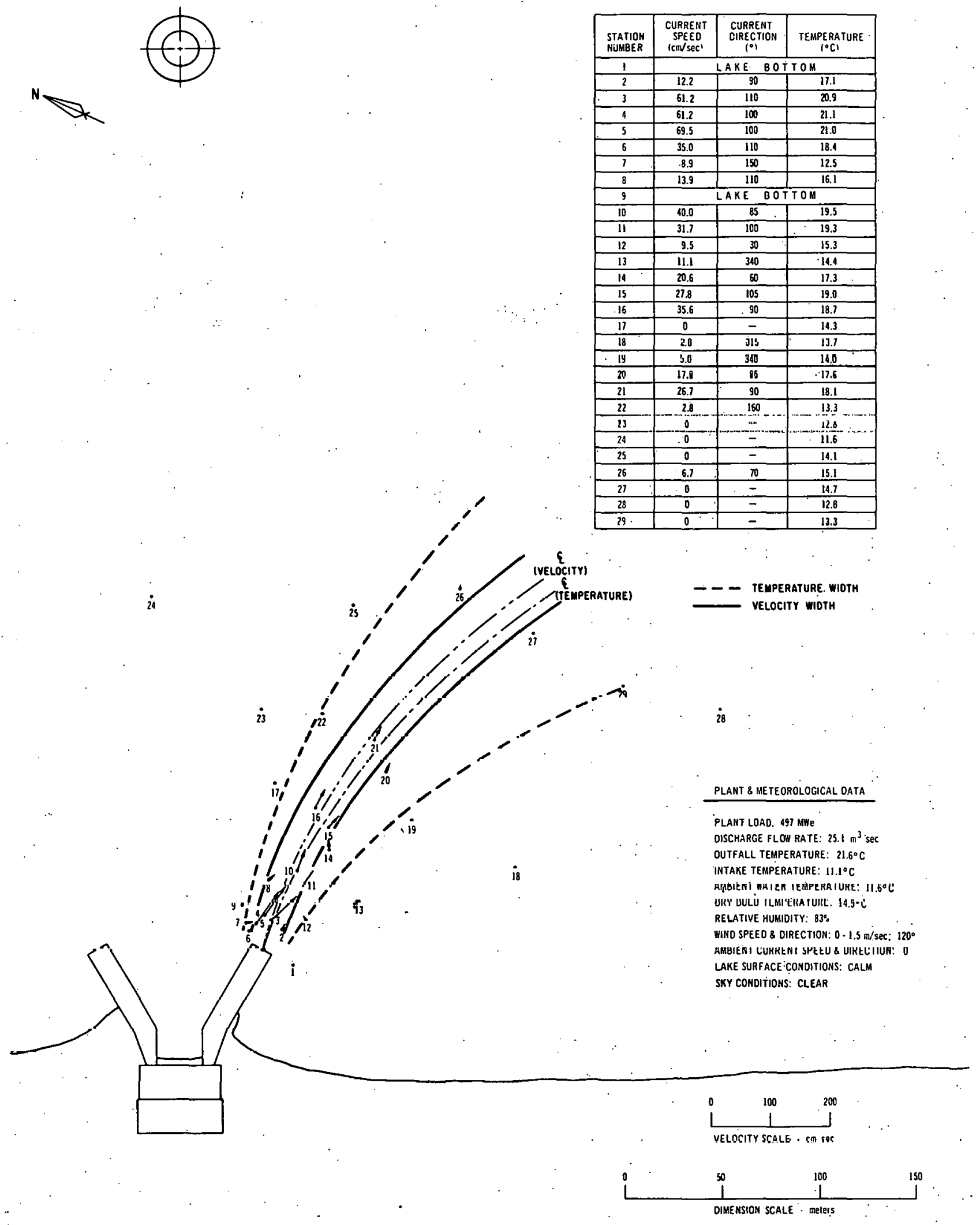

Fig. 17. Jet-regime Study for 2,0-m Depth at Point Beach Power Plant (Unit 1): May 23, 1972, 0945-1700 Hours. ANL Neg. No. 190-894. 


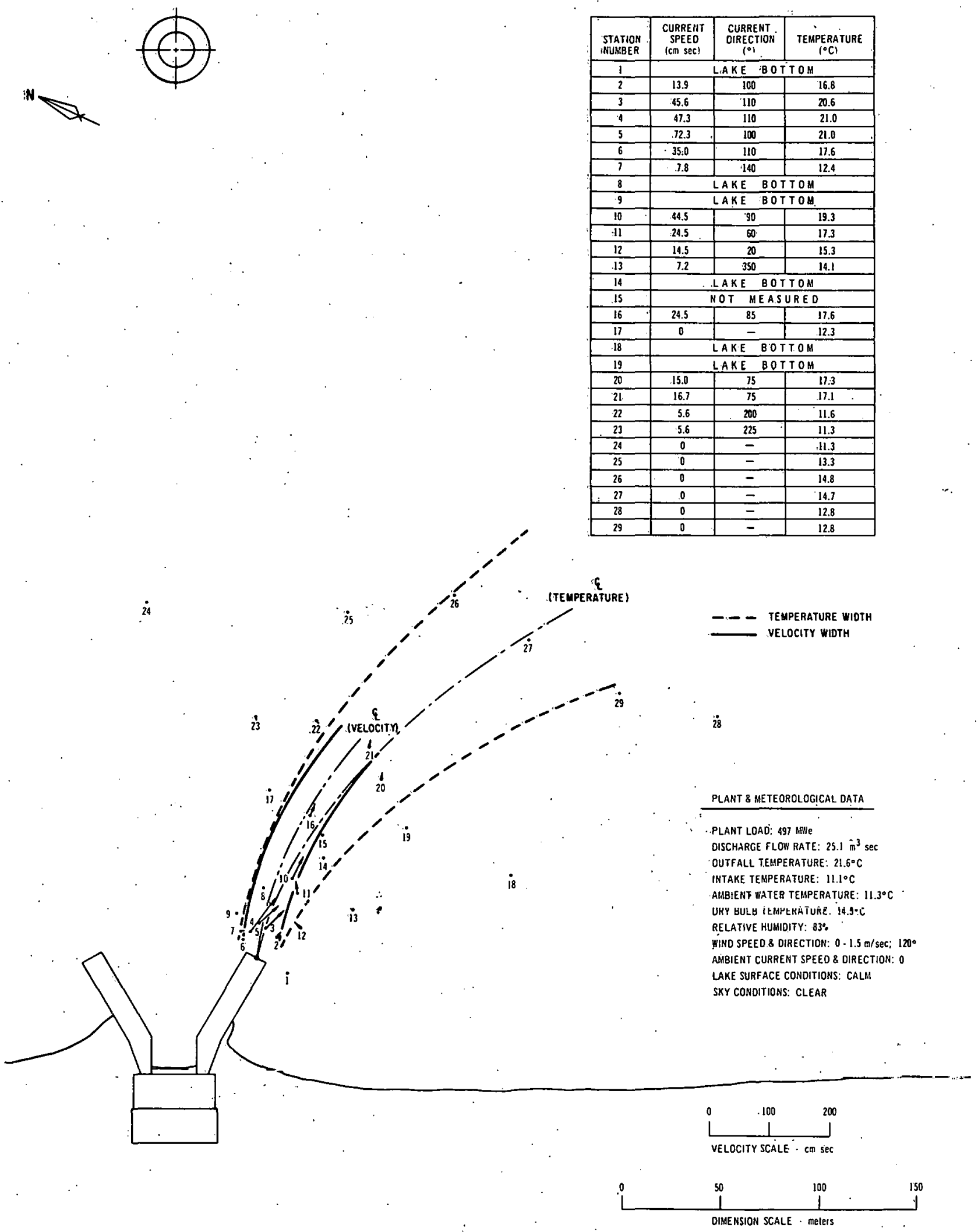

Fig. 18. Jet-regime Study for 2,5-m Depth at Pnint Beach Power Plant (Unit 1): May 23, 1972, 0945-1700 Hours. ANL Neg. No. 190-892. 


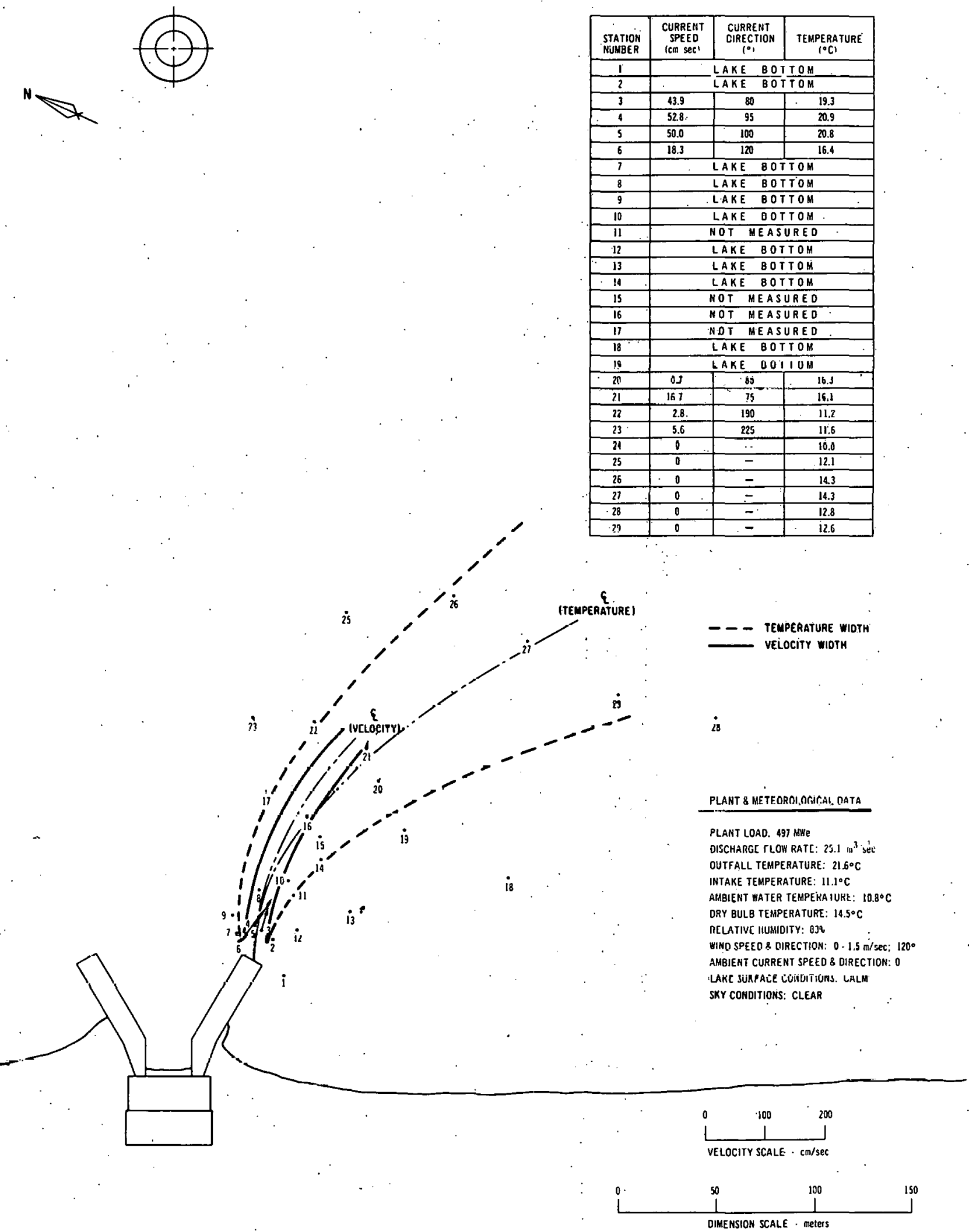

Fig. 19. Jet-regime Study for 3.0-m Depth at Point Beach Power Plant (Unit 1): May 23, 1972, 0945-1700 Hours. ANL Neg. No. 190-890. 
$N$

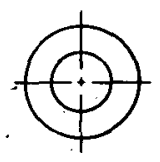

\begin{tabular}{|c|c|c|c|}
\hline $\begin{array}{l}\text { STATION } \\
\text { : NUMBER } \\
\end{array}$ & $\begin{array}{c}\text { CURRENT } \\
\text { SPEED } \\
(\mathrm{cm} / \mathrm{sec})\end{array}$ & $\begin{array}{l}\text { CURRENT } \\
\text { DIRECTION } \\
\left(0^{\circ}\right)\end{array}$ & $\begin{array}{l}\text { TEMPERATURE } \\
. \quad\left({ }^{\circ} \mathrm{C}\right) .\end{array}$ \\
\hline 1 & 19.5 & 180 & 17.0 \\
\hline 2 & 62.3 & 110 & 19.0 \\
\hline 3 & 67.8 & 95 & 20.2 \\
\hline 4 & 51.1 & 80 & 18.5 \\
\hline 5 & 17.8 & 65 & 16.7 \\
\hline 6 & 5.6 & $60^{\circ}$ & 16.5 \\
\hline 7 & 30.0 & 80 & $\begin{array}{lll}16.7 \\
\end{array}$ \\
\hline 18 & $44.5^{\circ}$ & 95 & 18.8 \\
\hline 9 & 51.1 & 110 & 19.2 \\
\hline 10 & 23.4 & 125 & 17.0 \\
\hline II & 8.9 & 135 & 17.0 \\
\hline 12 & 6.7 & 75 & 15.3 \\
\hline 13 & 26.1 & 115 & 16.3 \\
\hline 14 & 30.0 & 120 & 16.5 \\
\hline 15 & 24.4 & 140 & 16.0 \\
\hline 116 & 13.9 & 170 & 15.5 \\
\hline .17 & $11: 1$ & 145 & 15.7 \\
\hline
\end{tabular}

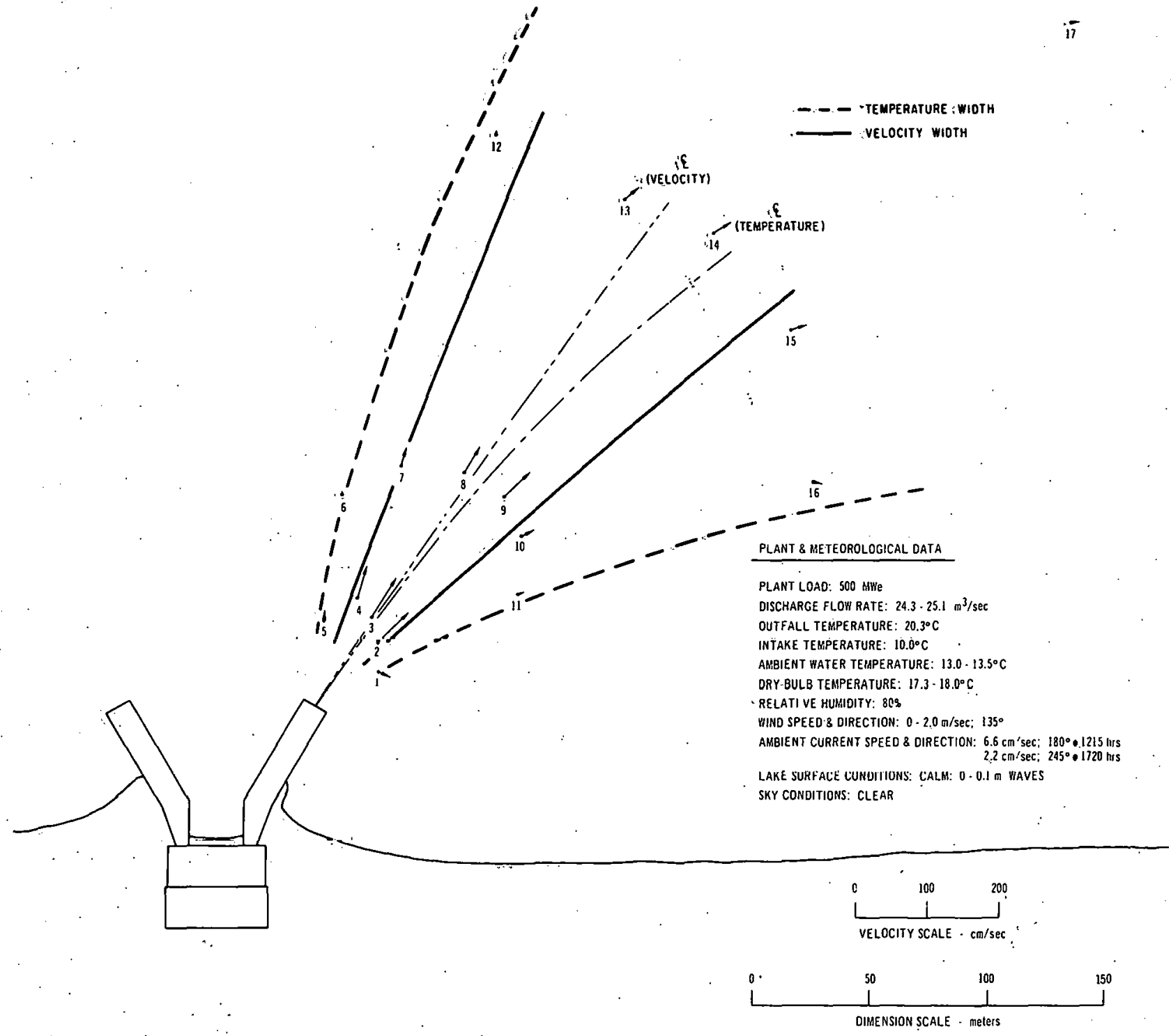

Flg. 20. Jet-reglme Study for 0.5-m Depth at Polnt Beach Power Plant (Unit 1):

July $13,1972,1308-17.06$ Hours. ANL Neg. No. 190-762 Rev. 2. 


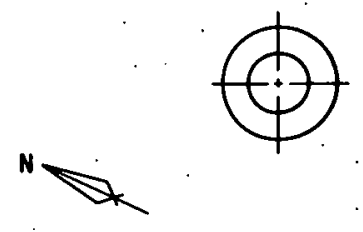

\begin{tabular}{|c|c|c|c|}
\hline $\begin{array}{c}\text { STATION } \\
\text { MUMBER }\end{array}$ & $\begin{array}{c}\text { CURRENT } \\
\text { SPEED } \\
(\text { Em/sec) }\end{array}$ & $\begin{array}{c}\text { CURRENT } \\
\text { ORECTION } \\
\left({ }^{\circ}\right)\end{array}$ & $\begin{array}{c}\text { TEMPERATURE } \\
\left({ }^{\circ} \mathrm{C}\right)\end{array}$ \\
\hline 1 & 12.2 & 115 & 16.7 \\
\hline 2 & 62.8 & 110 & 19.0 \\
\hline 3 & 60.0 & 95 & 20.0 \\
\hline 1 & 37.3 & 80 & 18.0 \\
\hline 5 & 14.5 & 130 & 16.7 \\
\hline 5 & 2.2 & 325 & 16.0 \\
\hline 1 & 20.6 & 90 & 15.5 \\
\hline 8 & 42.3 & 95 & 18.3 \\
\hline 9 & 40.0 & 110 & 18.3 \\
\hline 10 & 19.5 & 115 & 16.3 \\
\hline 11 & 15.0 & 160 & 16.2 \\
\hline 12 & 2.2 & 115 & 14.0 \\
\hline 13 & 16.7 & 120 & 15.0 \\
\hline 14 & 22.2 & 120 & 15.0 \\
\hline 15 & 16.7 & 145 & 14.7 \\
\hline 16 & 3.9 & 195 & 14.0 \\
\hline 11 & 10.6 & 143 & 19.7 \\
\hline
\end{tabular}

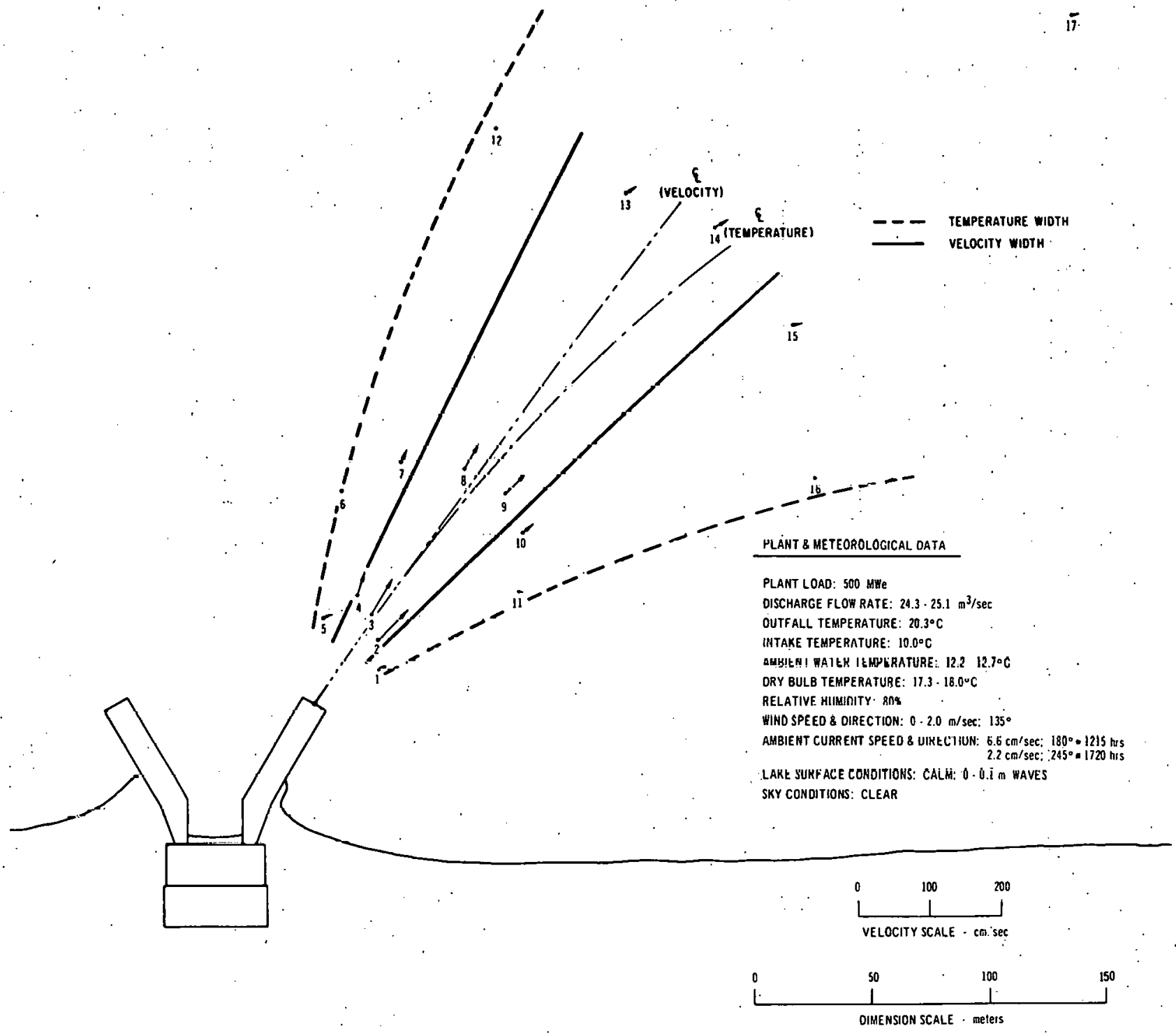

Fig. 21. Jet-regime Study for $1.0-\mathrm{m}$ Depth at Point Beach Power Plant (Unit 1): July 13, 1972, 1308-1706 Hours. ANL Neg. No. 190-900 Rev. 1. 


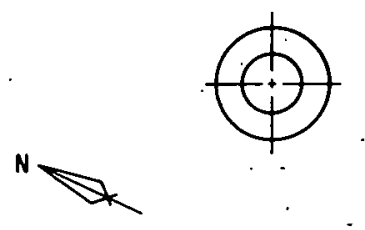

\begin{tabular}{|c|c|c|c|}
\hline $\begin{array}{c}\text { STATION } \\
\text { HUMBER }\end{array}$ & $\begin{array}{c}\text { CURRENT } \\
\text { SPEED } \\
(\text { (mm/sec) }\end{array}$ & $\begin{array}{c}\text { CURRENT } \\
\text { ORECTION } \\
\left({ }^{\circ}\right)\end{array}$ & $\begin{array}{c}\text { TEMPERATURE } \\
\left({ }^{\circ} \mathrm{C}\right)\end{array}$ \\
\hline 1 & 12.2 & 45 & 16.0 \\
\hline 2 & 32.2 & 110 & 17.0 \\
\hline 3 & 67.8 & 95 & 18.5 \\
\hline 1 & 40.0 & 90 & 18.5 \\
\hline 5 & 14.5 & 180 & 16.0 \\
\hline 6 & 0 & - & 15.5 \\
\hline 7 & 16.7 & 125 & 15.2 \\
\hline 8 & 36.7 & 95 & 17.7 \\
\hline 9 & 37.3 & 110 & 18.3 \\
\hline 10 & 11.1 & 115 & 16.0 \\
\hline 11 & 3.9 & 250 & 15.7 \\
\hline 12 & 6.7 & 145 & 12.5 \\
\hline 13 & 11.1 & 125 & 14.5 \\
\hline 11 & 13.3 & 130 & 14.0 \\
\hline 15 & 9.5 & 150 & 13.5 \\
\hline 16 & 0 & - & 12.7 \\
\hline 17 & 6.7 & 150 & 12.5 \\
\hline
\end{tabular}

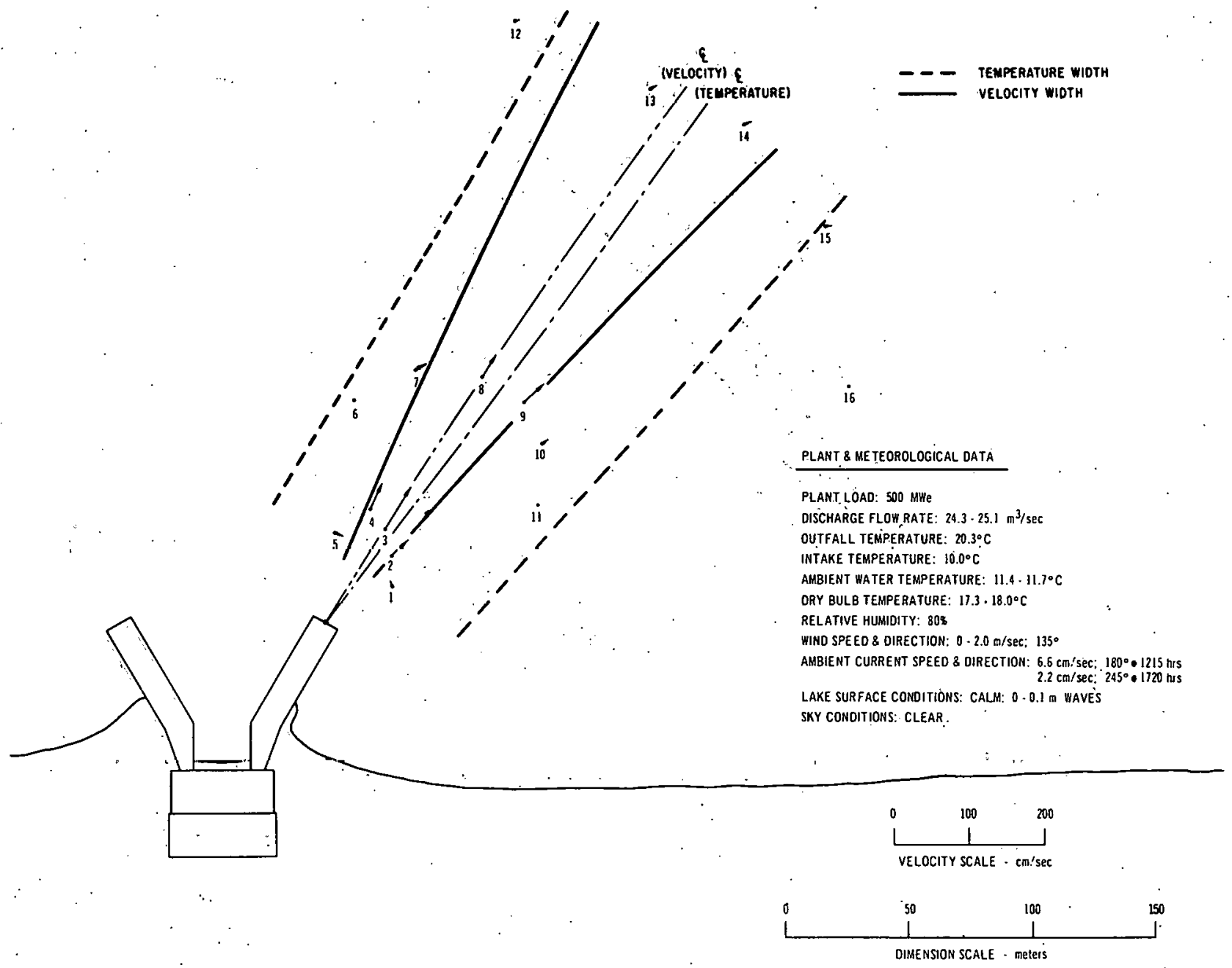

Fig. 22. Jer-reglme Sruidy for 1:5-m Depth ar Pofnr, Beach Power Planr (Unfr 1): July 13, 1972; 1308-1706 Hours. : ANL Neg. No. 190-903 Rev. 1. 


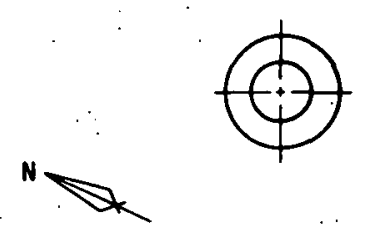

\begin{tabular}{|c|c|c|c|}
\hline $\begin{array}{l}\text { STATION } \\
\text { NUMEER }\end{array}$ & $\begin{array}{l}\text { CURRENT } \\
\text { SPEED } \\
(\mathrm{em} / \mathrm{sec})\end{array}$ & $\begin{array}{c}\text { CURRENT } \\
\text { OIRECTIOH } \\
\left.()^{\circ}\right) .\end{array}$ & $\begin{array}{l}\text { TEMPERATURE } \\
\left({ }^{\circ} \mathrm{C}\right)\end{array}$ \\
\hline 1 & 16.7 & 25 & 14.5 \\
\hline 2 & 40.0 & 110 & 18.5 \\
\hline 3 & 77.8 & 95 & 19.5 \\
\hline 1 & 30.0 & 100 & 17.5 \\
\hline 5 & \multicolumn{3}{|c|}{ LAKE BOTTOM } \\
\hline 6 & 11.1 & 185 & 14.3 \\
\hline 1 & 13.9 & 170 & 13.5 \\
\hline 8 & 33.4 & 95 & 17.7 \\
\hline$\dot{g}$ & 28.9 & 125 & 16.5 \\
\hline 10 & 6.7 & 40 & 15.0 \\
\hline II & \multicolumn{3}{|c|}{ LAKE BOTTOM } \\
\hline 12 & 5.6 & 180 & 11.5 \\
\hline 13 & 8.3 & 135 & 14.0 \\
\hline 11 & 11.1 & 150 & 13.3 \\
\hline 15 & 1.1 & 170 & 12.3 \\
\hline 16 & 5.6 & 320 & 11.7 \\
\hline 17 & 1.1 & 180 & 11.5 \\
\hline
\end{tabular}

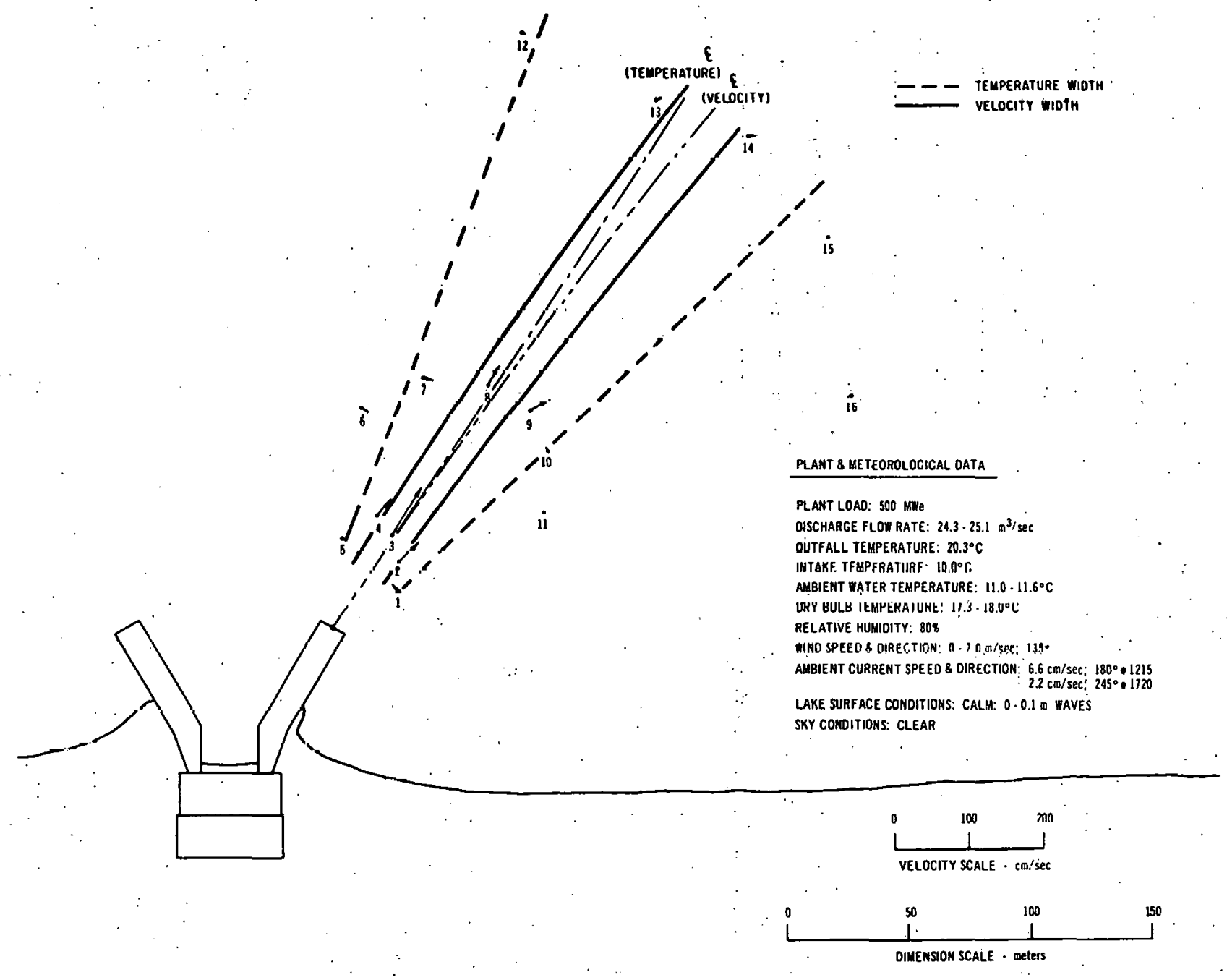

Fig. 23. Jet-regime Study for 2.0-m Depth at Point Beach Power Plant (Unit.1): July 13, 1972, 1308-1706 Hours. ANL Neg. No. 190-905 Rev. 1. 
N

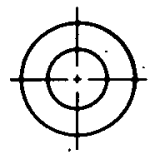

\begin{tabular}{|c|c|c|c|}
\hline $\begin{array}{l}\text { STATION } \\
\text { MUMBER }\end{array}$ & $\begin{array}{c}\text { CURRENT } \\
\text { SPEED } \\
(\mathrm{Cm} / \mathrm{sec})\end{array}$ & $\begin{array}{l}\text { CURRENT } \\
\text { OIRECTION } \\
(\odot)\end{array}$ & $\begin{array}{l}\text { TEMPERATURE } \\
\left({ }^{\circ} \mathrm{C}\right)\end{array}$ \\
\hline 1 & 20.0 & 15 & 12.7 \\
\hline 2 & 23.4 & 70 & 15.0 \\
\hline 3 & 51.1 & 95 & 19.0 \\
\hline 1 & \multirow{2}{*}{\multicolumn{3}{|c|}{ LAKE BOTIOM }} \\
\hline 5 & \multicolumn{2}{|c|}{ LAKE BOTTOM } & \\
\hline 6 & 12.2 & 180 & 12.0 \\
\hline 7 & 13.9 & 195 & 12.0 \\
\hline 8 & 31.7 & 95 & 17.7 \\
\hline 9 & 13.9 & 150 & 15.5 \\
\hline 10 & 10.0 & 30 & 11.7 \\
\hline 11 & \multicolumn{3}{|c|}{ LAKE BOTIOM } \\
\hline 12 & 3.9 & 180 & 11.3 \\
\hline 13 & 3.9 & $175^{\circ}$ & 12.5 \\
\hline 14 & 5.6 & 190 & 12.0 \\
\hline 15 & 1.1 & 230 & 11.3 \\
\hline 16 & 5.6 & 330 & 11.0 \\
\hline 17 & 0 & - & 10.7 \\
\hline
\end{tabular}

i7

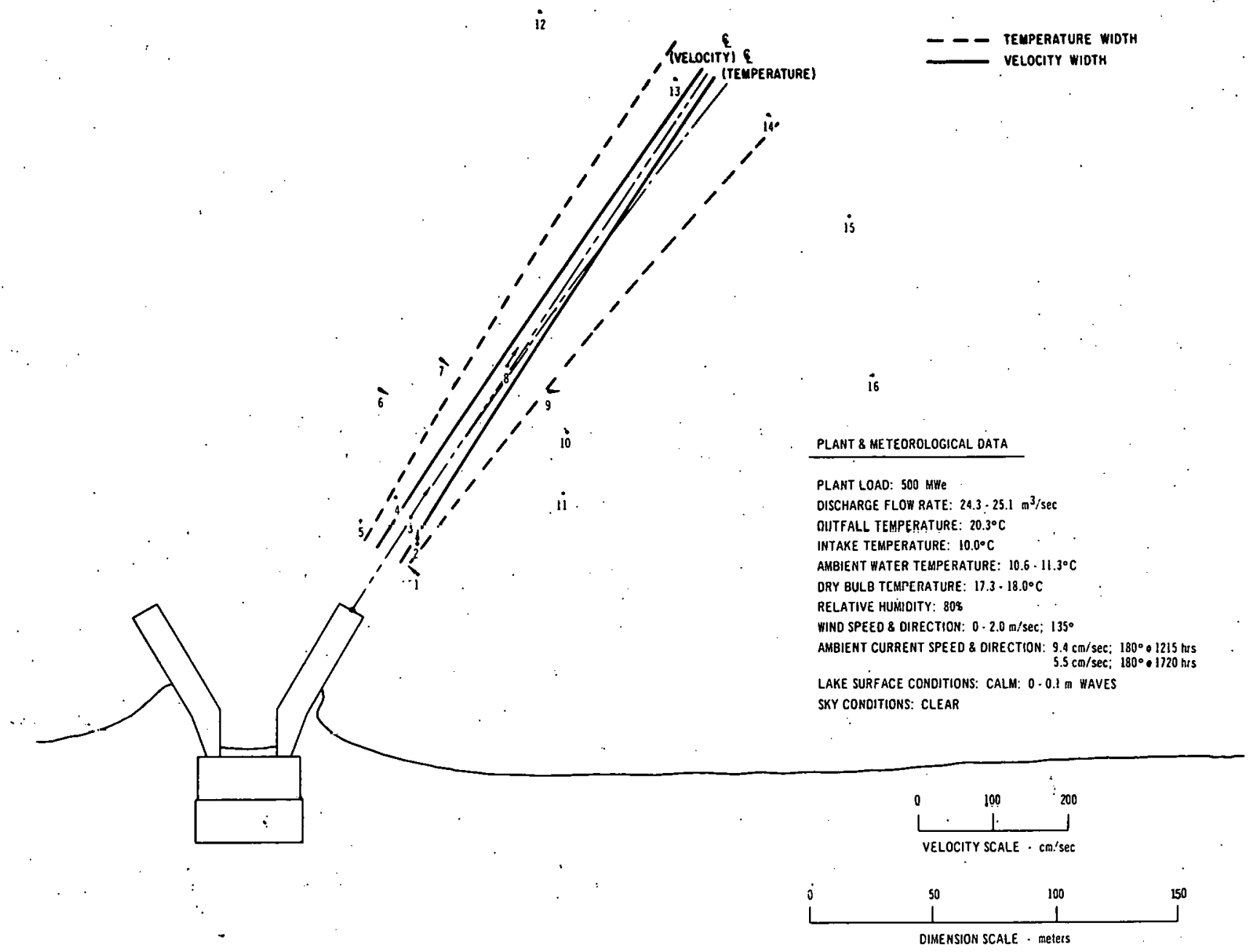

Eig. 24. Jet-regime Study for 2.5-m Depth at Point Beach Puwer Plall (Unit 1): July 13, 1972, 1308-1706 Hours. ANL Neg. No. 190-896 Rev. 1. 


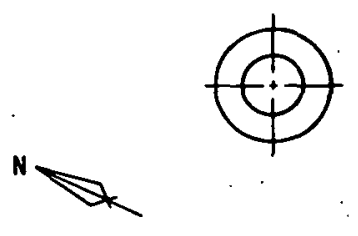

\begin{tabular}{|c|c|c|c|}
\hline $\begin{array}{l}\text { STATION } \\
\text { MUIBSER }\end{array}$ & $\begin{array}{l}\text { CURRENT } \\
\text { SPEEO } \\
\text { IEm seCI }\end{array}$ & $\begin{array}{l}\text { CURRENT } \\
\text { OIRECTION } \\
(=1)\end{array}$ & $\begin{array}{c}\text { TEMPERATURE } \\
\left({ }^{\circ} \mathrm{Cl}\right)\end{array}$ \\
\hline 1 & \multicolumn{3}{|c|}{ LAKE BOTTOU } \\
\hline 2 & 15.0 & 35 & 15.0 \\
\hline 3 & \multicolumn{3}{|c|}{ LAKE BOTTOM } \\
\hline 1 & \multicolumn{3}{|c|}{ LAKE BOTTOM } \\
\hline 5 & \multicolumn{3}{|c|}{ LAKE BOTTOM } \\
\hline 6 & \multicolumn{3}{|c|}{ LAKE BOTTOH } \\
\hline 7 & \multicolumn{3}{|c|}{ LAKE BOTTOM } \\
\hline 8 & \multicolumn{3}{|c|}{ LAKE BOTTOM } \\
\hline 9 & \multicolumn{3}{|c|}{ LAKE BOTTOM } \\
\hline 10 & \multirow{2}{*}{\multicolumn{3}{|c|}{ LAKE BOTTOM }} \\
\hline II & & & \\
\hline 12 & 2.8 & 185 & 11.1. \\
\hline 13 & 7.2 & 205 & 11.5 \\
\hline 14 & 5.6 & 200 & 11.0 \\
\hline 15 & 1.1 & 250 & 11.0 \\
\hline 16 & 3.9 & 330 & 11.0 \\
\hline 17 & 0 & $=$ & 10.3 \\
\hline
\end{tabular}

iz

i)

ii

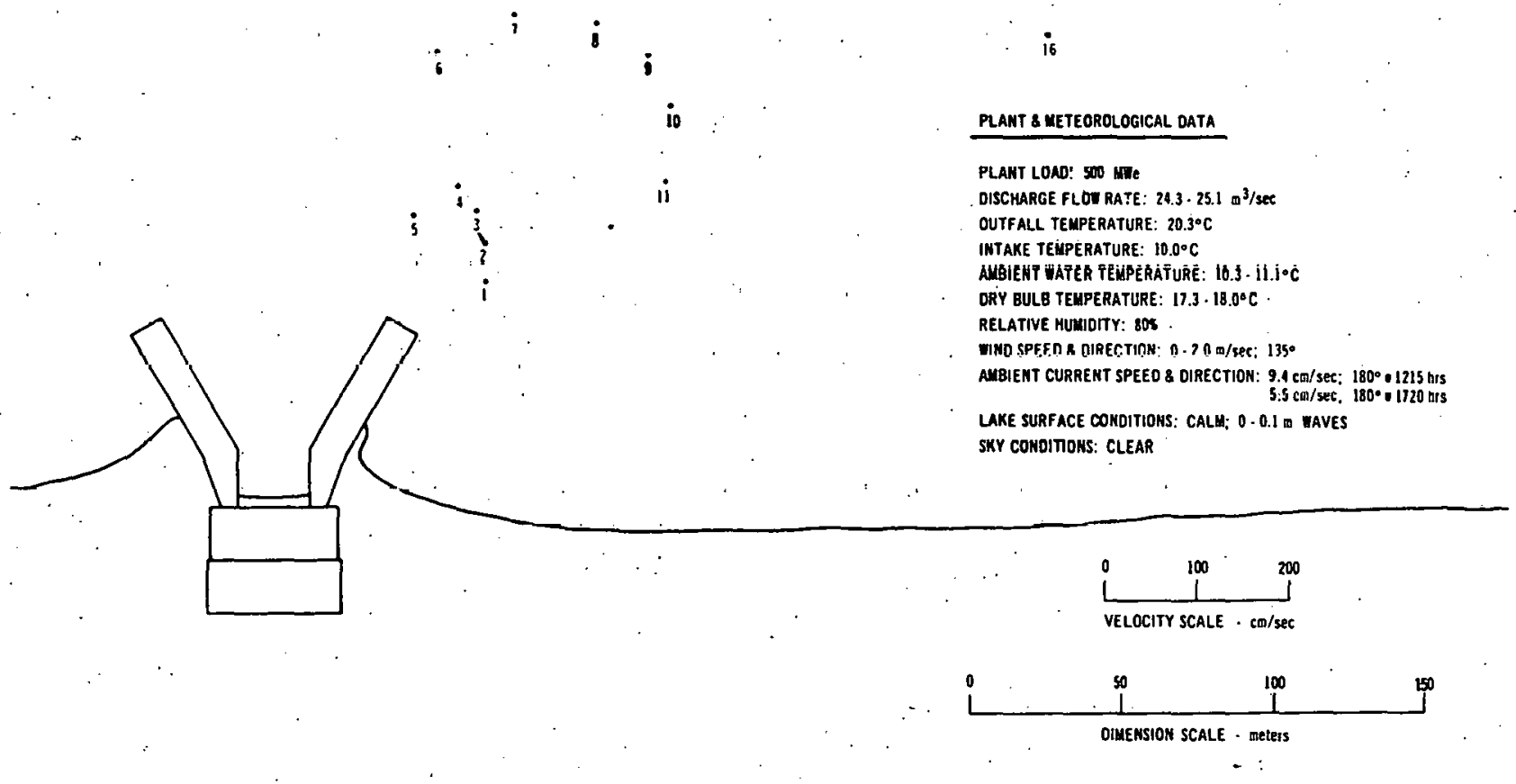

Fig. 25. Jet-regime Study for 3.0-m Depth at Point Beach Power Plant (Unit 1): July 13, 1972, 1308-1706 Hours: ANL Neg. No. 190-875 Rev. 1. 


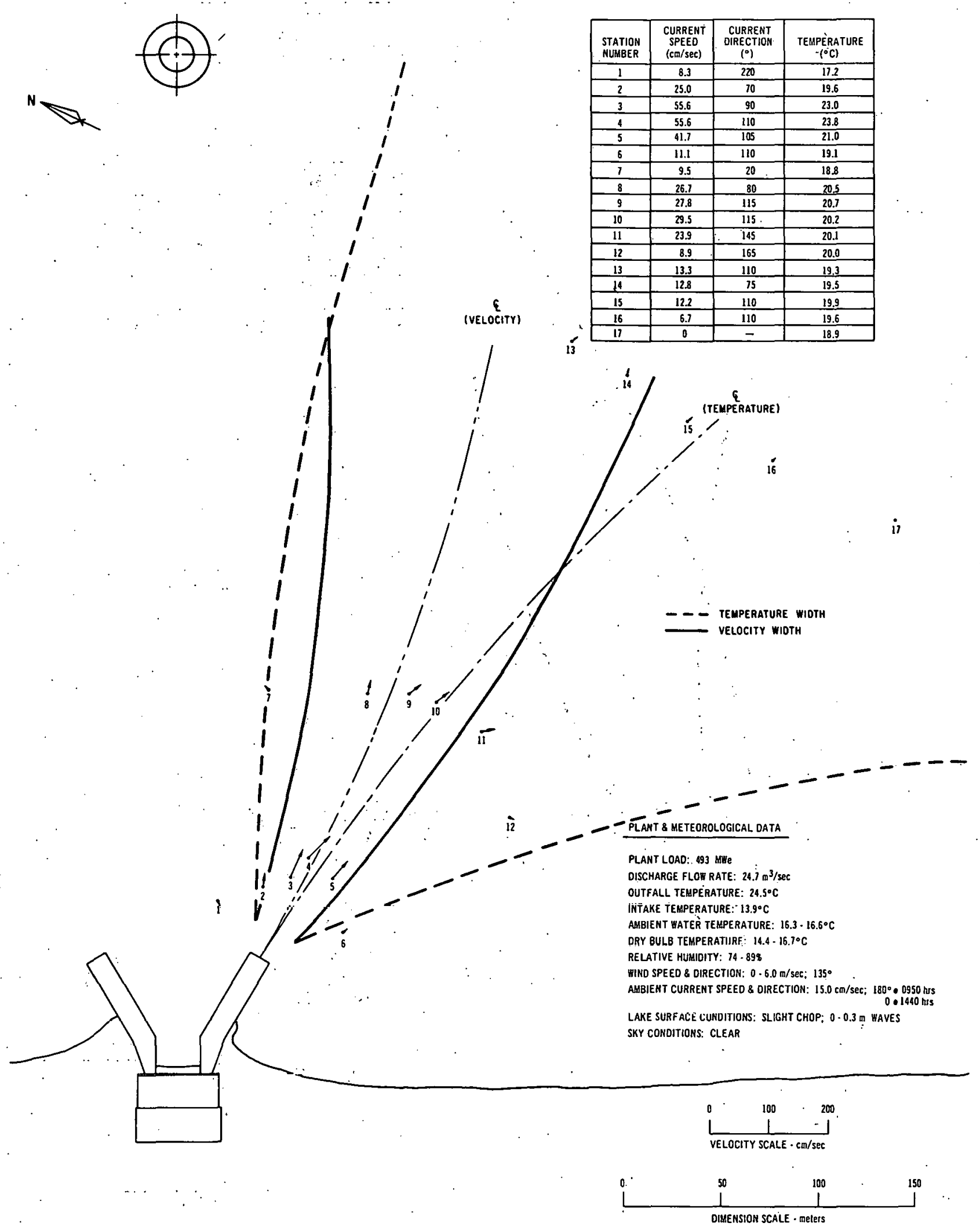

Fig. 26. Jet-regime Study for $0.5-\mathrm{m}$ Depth at Point Beach Power Plant (Junit 1): September $9,1972,1045-1420$ Hours. ANL Neg. No. 190-763 Rev. 1. 


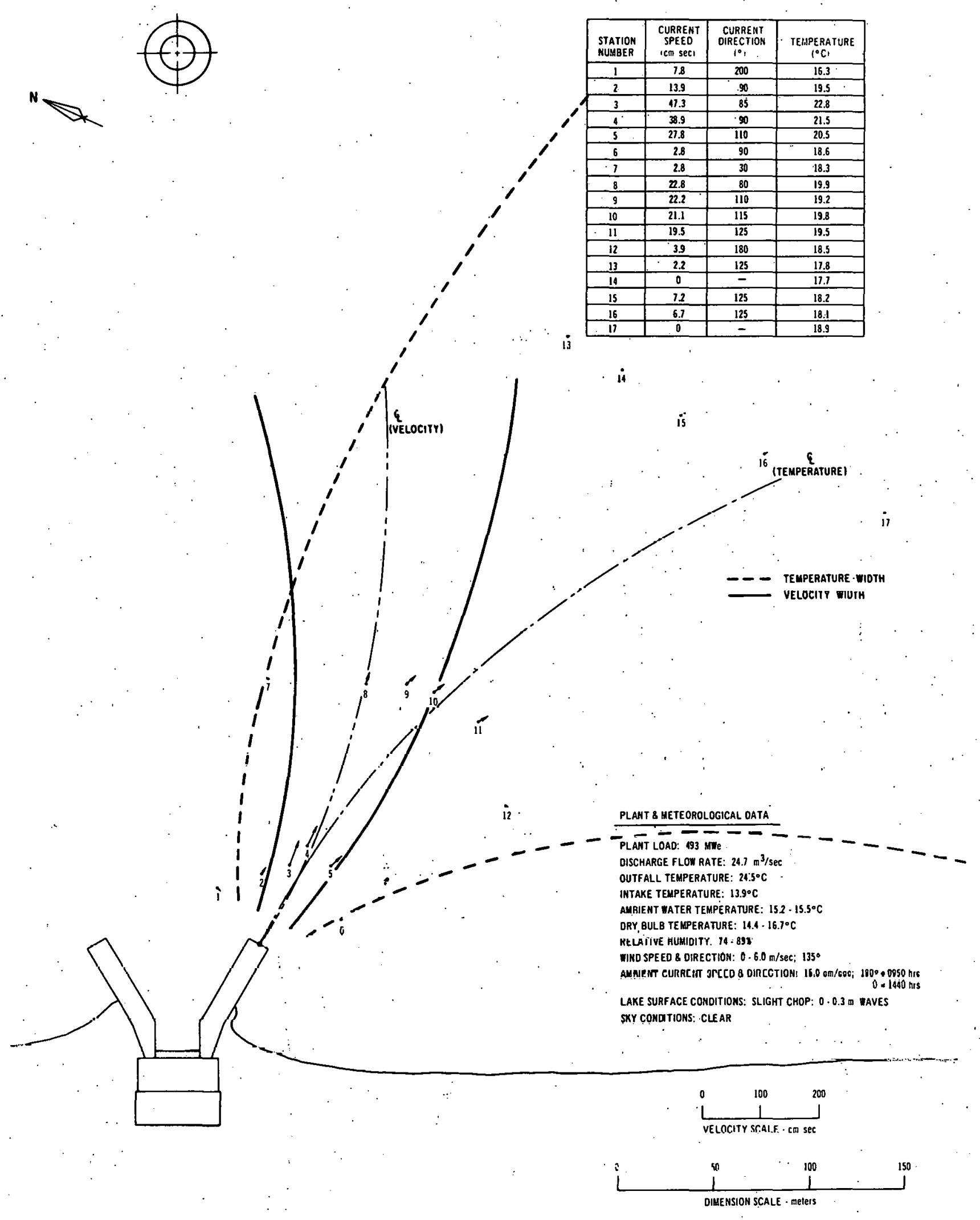

Fig: 27. Jet-regime Study for 1.0-m Depth at Point Beach Power Plant (Unit 1): September 9, 1972, 1045-1420. Hours. ANL Neg. No. 190-904. 


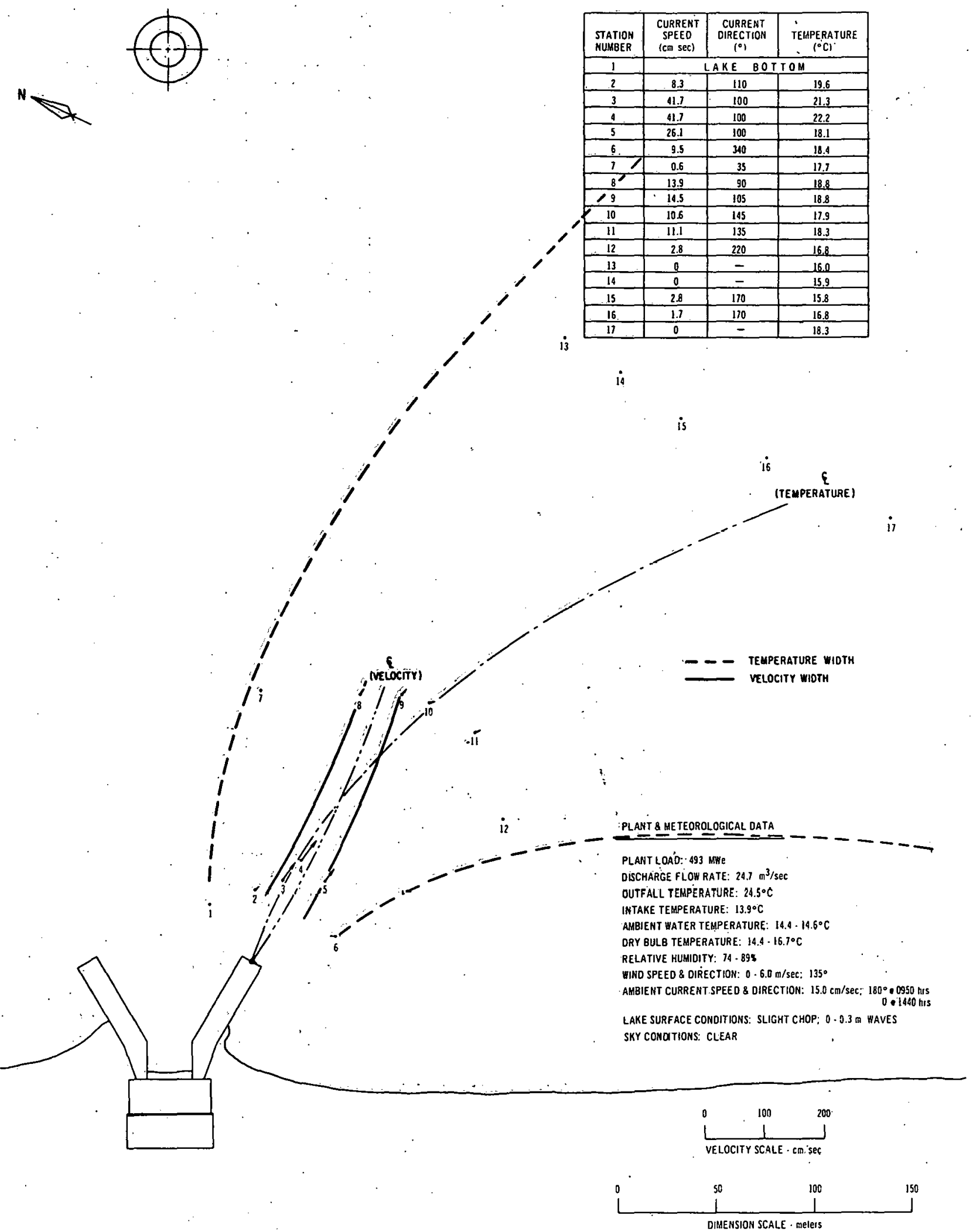

Fig. 28. Jet-regime-Study for 1.5-m Depth at Point Beach Power Plant (Unit 1): .. September $9,1972,1045-1420$ Hours." ANL Neg. No. 190-897. 


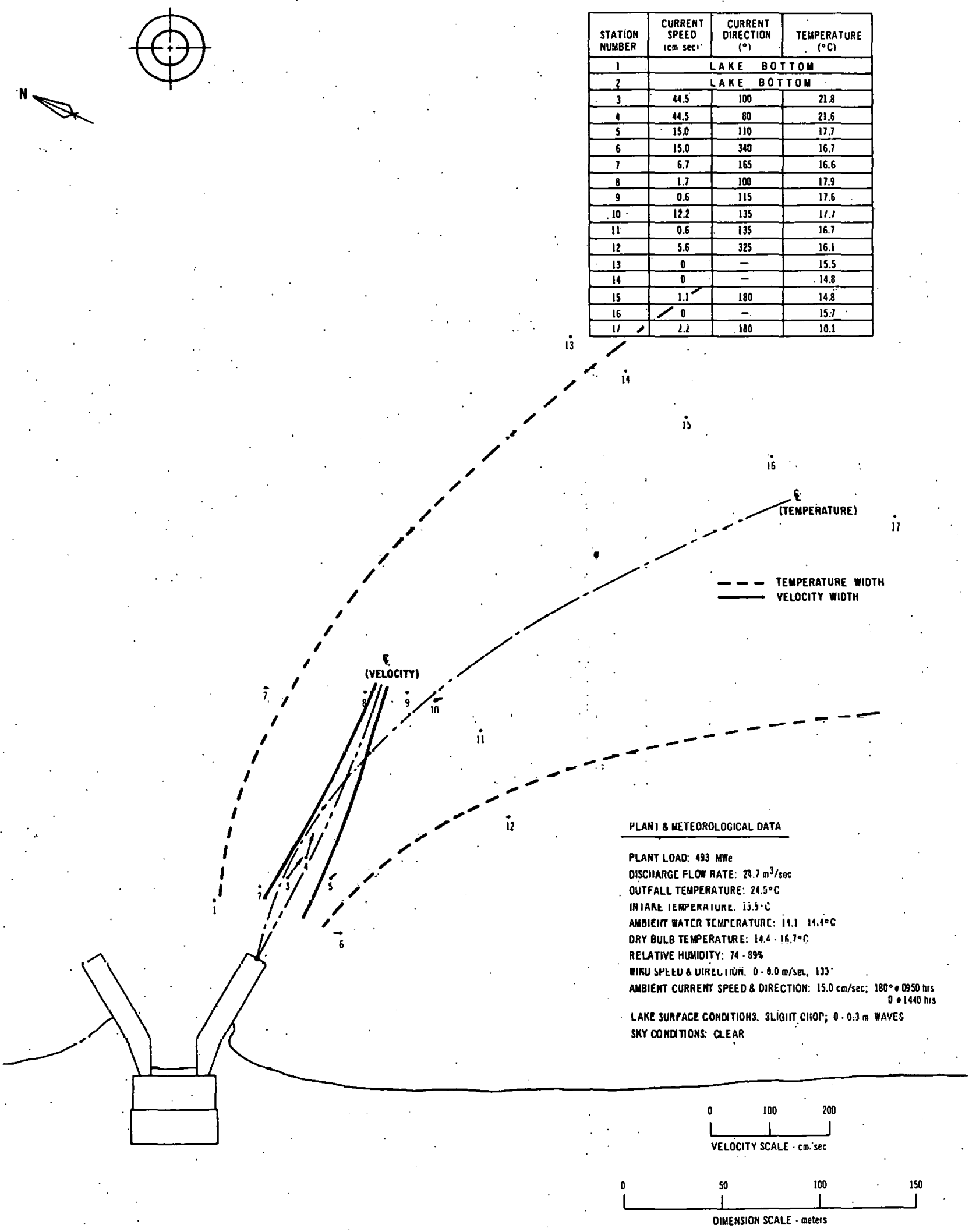

Fig. 29. Jet-regime Study for 2.0-m Depth at Point Beach Power Plant (Unit 1): September $9,1972,1045-1420$ Hours. ANL Neg. No. 190-902. 


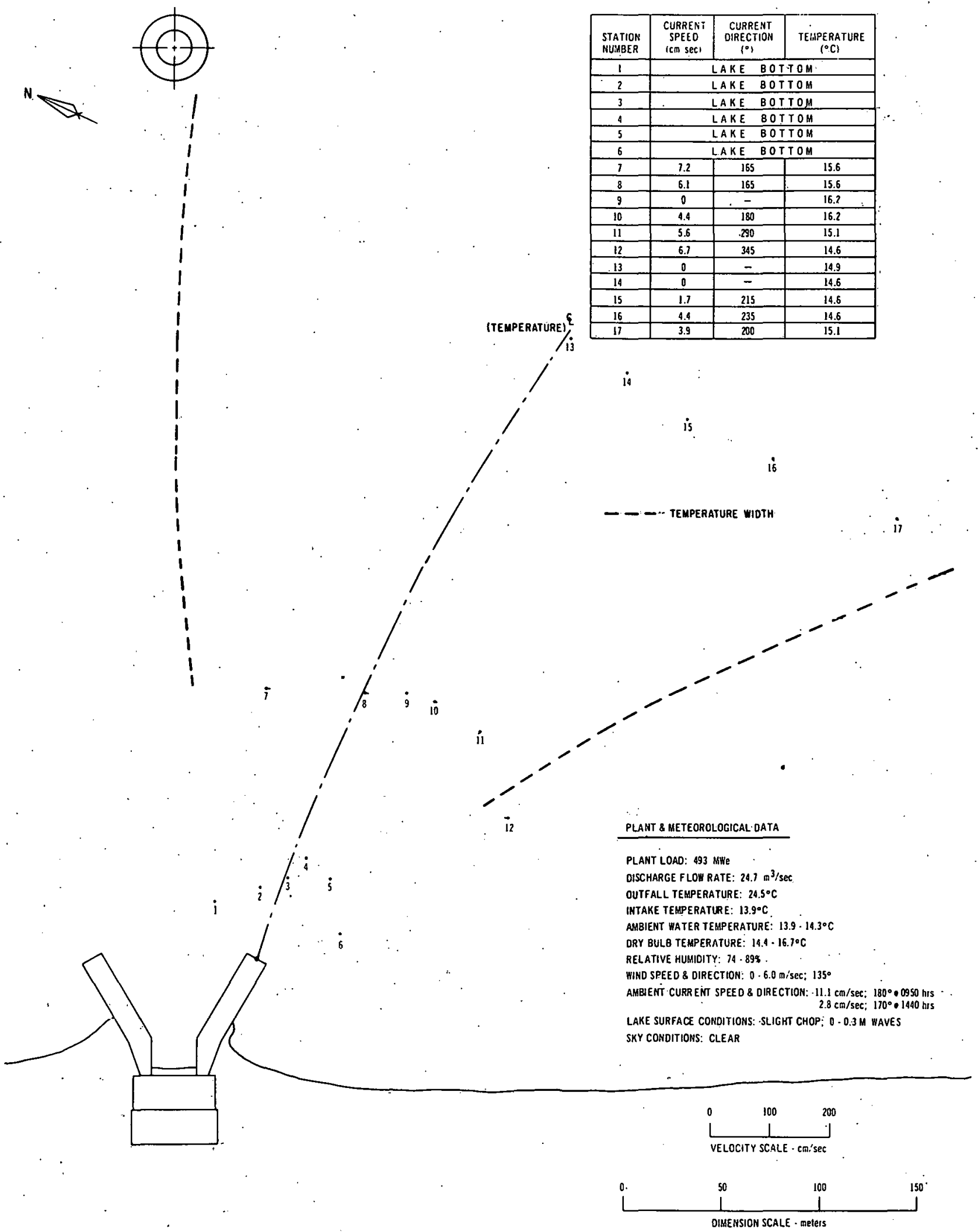

Fig. 3n. Jet-regime Study for 2.5-m Depth at Point Beach Power Plant (Unit 1): September $9,1972,1045-1420$ Hours. ANL Neg. No. 190-885. 


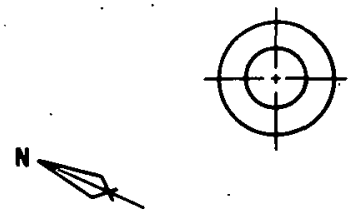

is

\begin{tabular}{|c|c|c|c|}
\hline $\begin{array}{l}\text { STATION } \\
\text { MUMEER }\end{array}$ & $\begin{array}{l}\text { CURRENT } \\
\text { SPEED } \\
\text { (cm/sec) }\end{array}$ & $\begin{array}{l}\text { CURRENT } \\
\text { OIRECTIOK } \\
\left(0^{\circ}\right)\end{array}$ & $\begin{array}{l}\text { TEMPERATURE } \\
\left({ }^{\circ} \mathrm{C}\right)\end{array}$ \\
\hline 1 & \multicolumn{3}{|c|}{ LAKE BOTTOM } \\
\hline 2 & \multicolumn{3}{|c|}{ LAKE BOTTOM } \\
\hline 3 & \multicolumn{3}{|c|}{ LAKE BOTTOM } \\
\hline 4 & \multicolumn{3}{|c|}{ LAKE BOTTOM } \\
\hline 5 & \multirow{2}{*}{\multicolumn{3}{|c|}{$\begin{array}{ll}\text { LAKE } & \text { BOTTOM } \\
\text { LAKE } & \text { BOTTOM }\end{array}$}} \\
\hline 6 & & & \\
\hline 7 & 7.2 & 180 & 14.9 \\
\hline 8 & \multicolumn{3}{|c|}{ LAKE BOTTOM } \\
\hline 9 & \multicolumn{3}{|c|}{ LAKE BOTTOM } \\
\hline 10 & \multicolumn{3}{|c|}{ LAKE BOTTOM } \\
\hline II & \multirow{2}{*}{\multicolumn{3}{|c|}{ 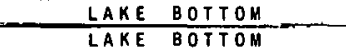 }} \\
\hline 12 & LAKE BOTTOM & & \\
\hline 13 & 0 & $\because \quad-$ & 14.6 \\
\hline 14 & 0 & $=$ & 19.5 \\
\hline 15 & 1.7 & 245 & 11.5 \\
\hline 16 & 2.8 & 235 & 10.5 \\
\hline 17 & 0 & - & 14.5 \\
\hline
\end{tabular}

i

is

i6

i7

i i i io

ii

PLANI \& ME IEOKOLOGICAL DATA

P.LANT LOAD: 493 MPRe

DISCHARGE FLOW RATE: $24.7 \mathrm{~m}^{3} / \mathrm{sec}$

OUTFALL TEMPERATURE: $24.5^{\circ} \mathrm{C}$

IHTAKE TLMREAATURE; 1?00\%,

AMPJFET WATFR TFMPFRATIIRF: $13.8-14.3^{\circ} \mathrm{C}$

ORY GULB TEMPERATURE: $14.1 \cdot 16.7^{\circ} \mathrm{C}$

RELATIVE HUMIOITY: 74 : 893

WIIO ORCCD 8 DINCOTIOH: $6.0 \mathrm{~m} / \mathrm{ags}$; 176

AMBIENT CURRENT SPEED \& OLRECTIOH: $11.1 \mathrm{~cm} / \mathrm{sec}$; $180^{\circ} \cdot 1215 \mathrm{mts}$ $2.8 \mathrm{~cm} / \mathrm{sec}: 170 \circ 1440 \mathrm{hrs}$

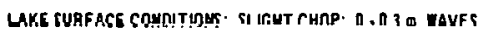

SKY COMOITIONS: CLEAR
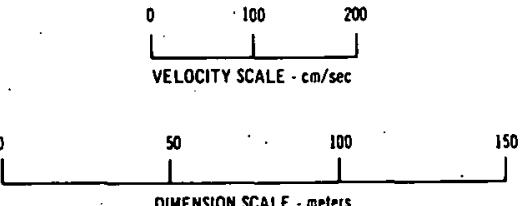

Fig. 31. Jet-regime Study for 3.0-m Depth at Point Beach Power Plant (Unit 1): September 9, 1972, 1045-1420 Hours. ANL Neg. No. 190-888. 


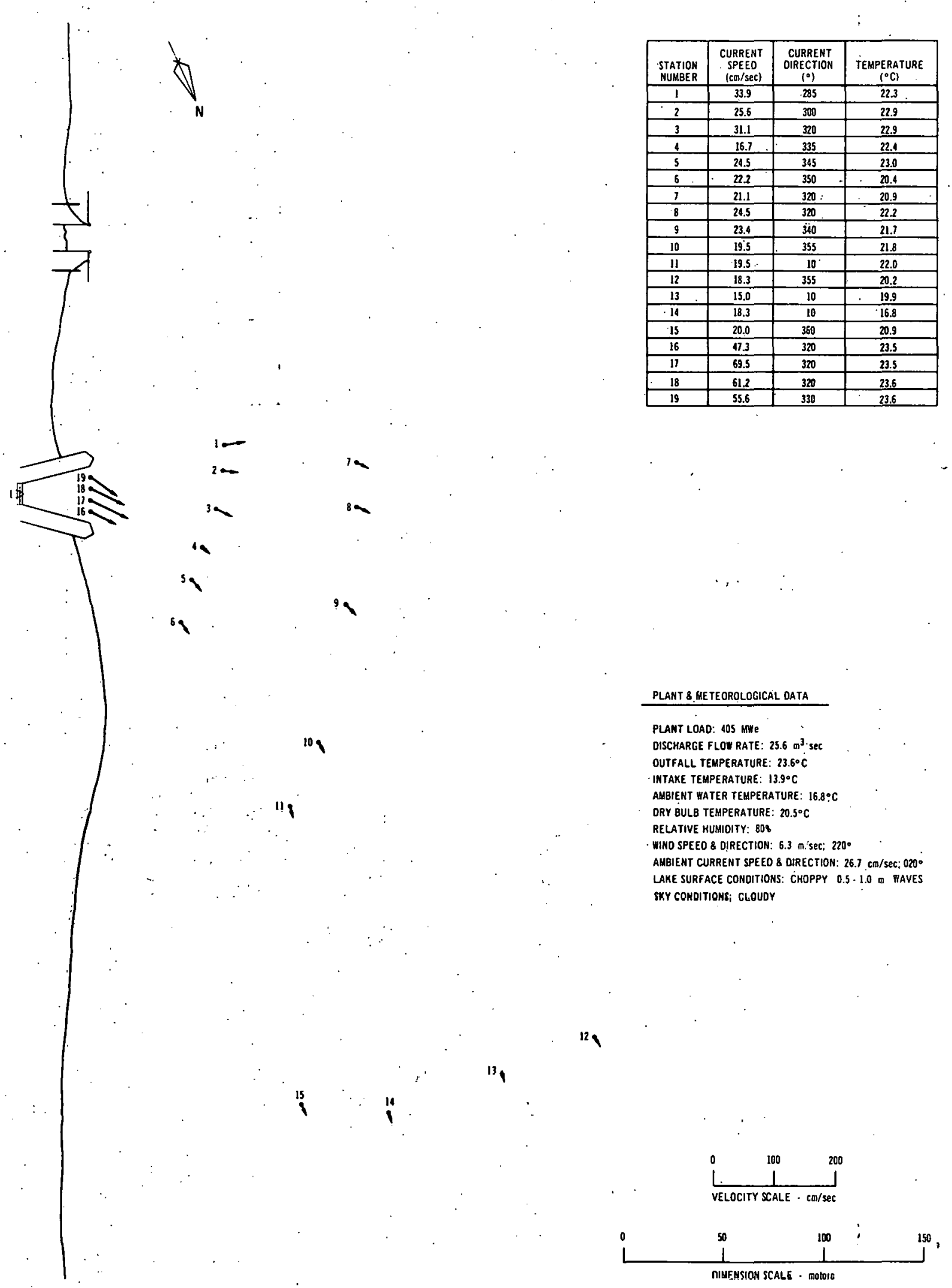

Fig. 32. Jet-regime Study for $0.5-\mathrm{m}$ Depth at Palisades Power Plant: June 14;1972,1000-1348. Hours: ANL Neg. No. 190-757 Rev. 1. 


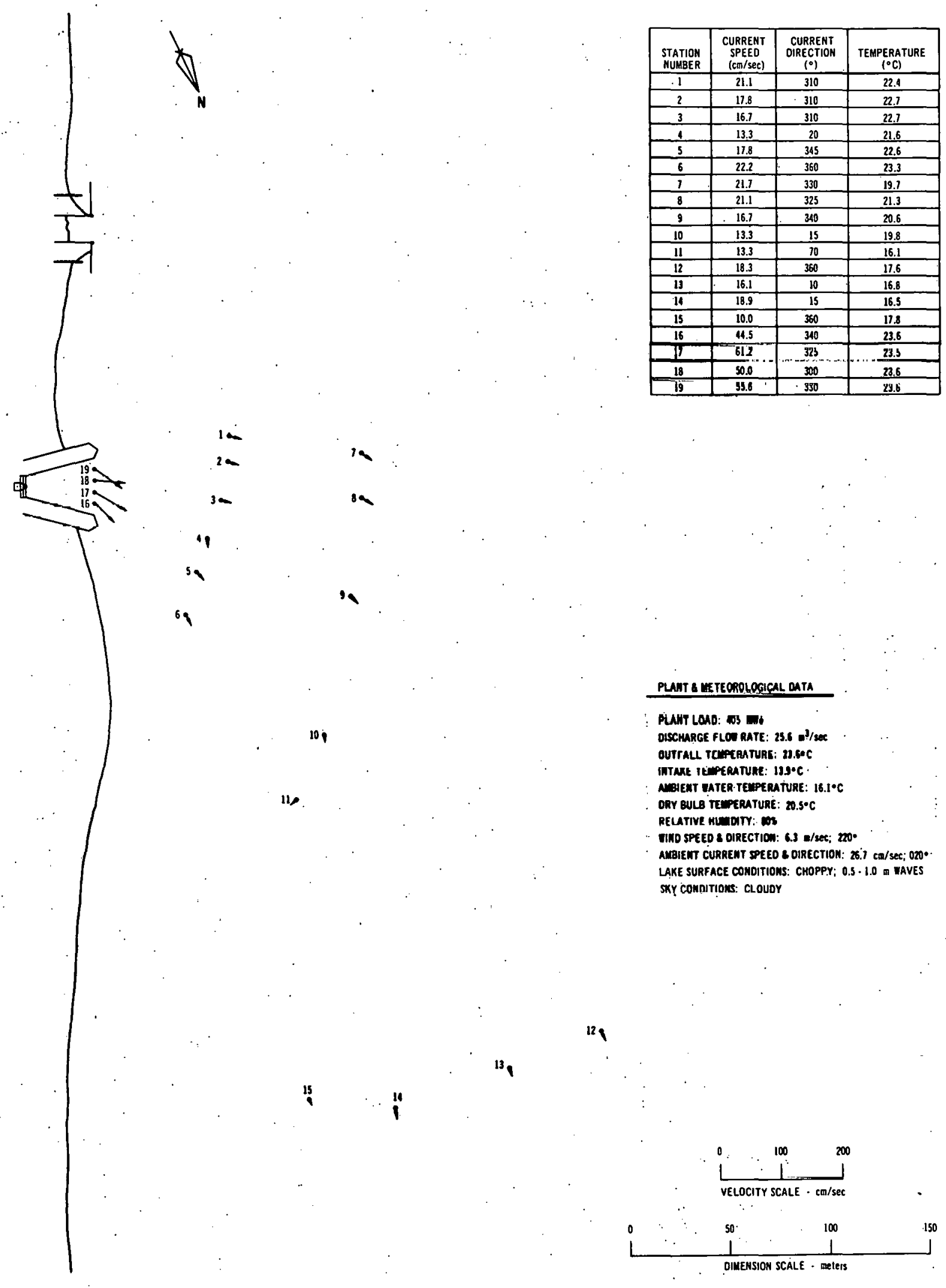

Fig. 33. Jet-regime Study for $1.0-\mathrm{m}$ Depth at Palisades Power Plant: June 14, 1972, 1000-1348 Hours. ANL Neg. No. 190-872. 


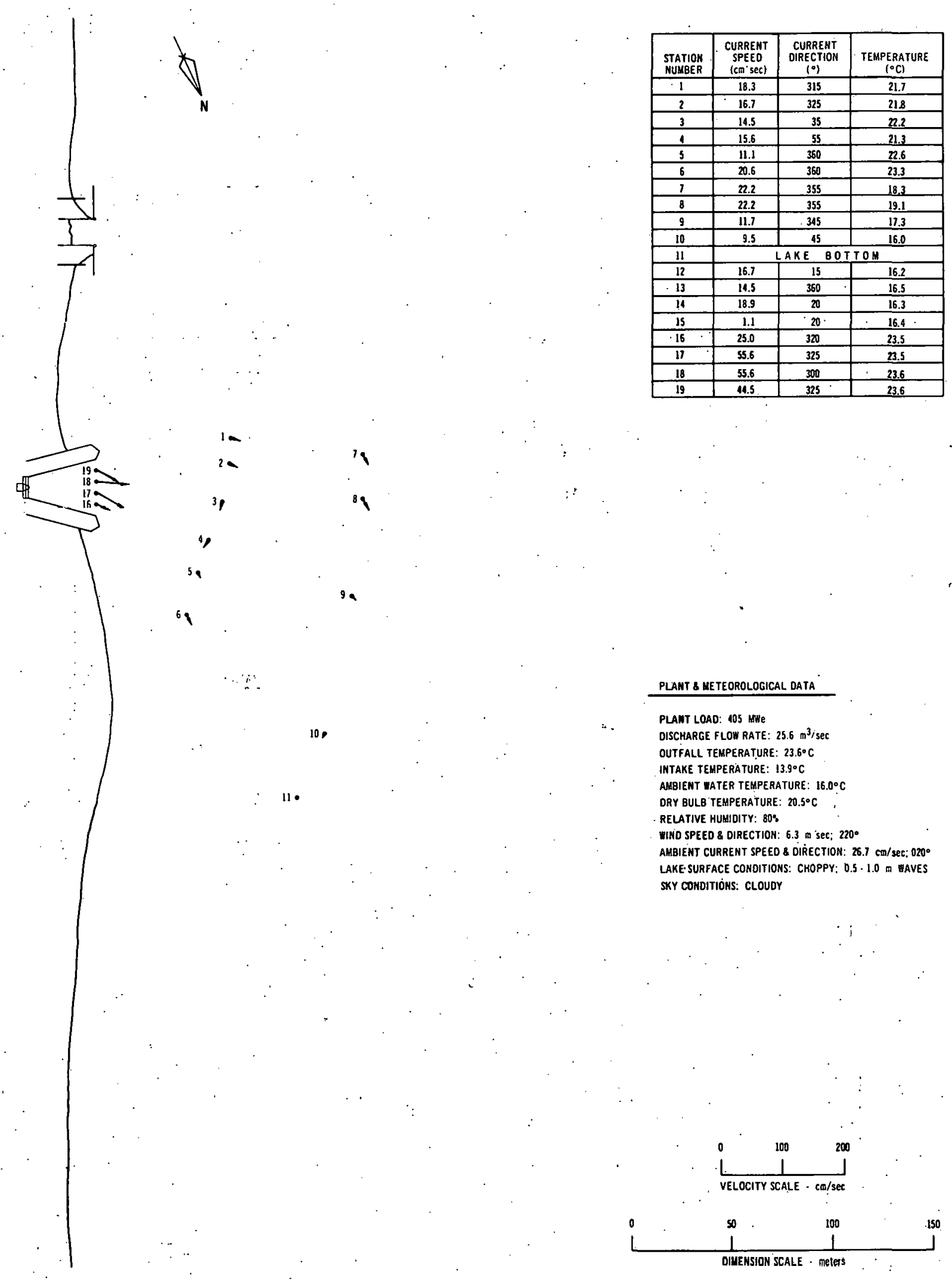

Fig. 34. Jet-regime Study for $1.5-\mathrm{m}$ Depth at Palisades Power Plant: Juile $14,1972,1000-1340$ Hours. ANL Neg. No. $100-870$. 


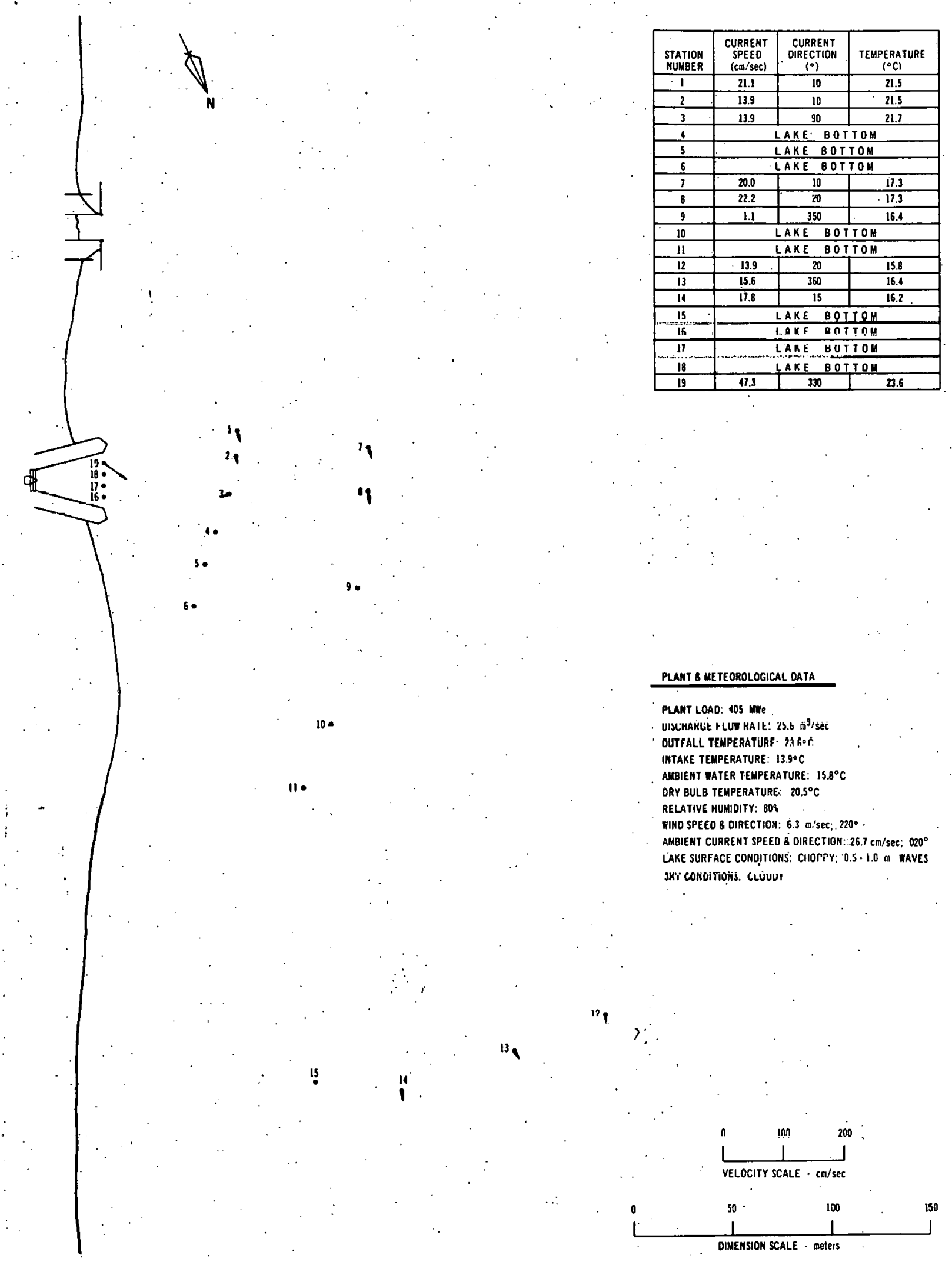

Fig. 35. Jet-regime Study for $2.0-\mathrm{m}$ Depth at Palisades Power Plant: June 14, 1972, 1000-1348 Hours. ANL Neg. No. 190-877 Rev. 1. 


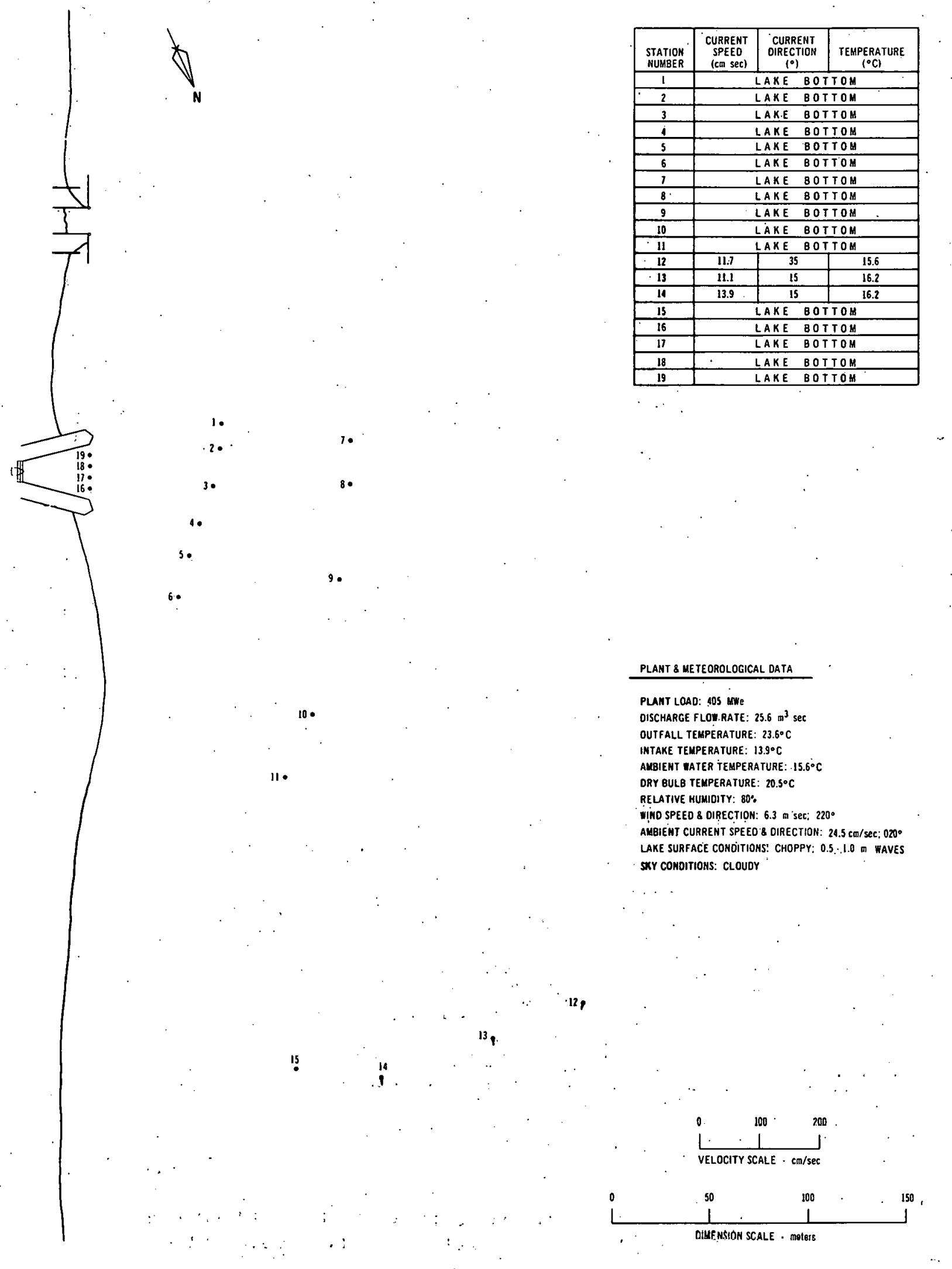

Fig. 36. Jet-regime Study for $2.5-\mathrm{m}$ Depth at Palisades Power Plant: June 14, 1972, 1000-1348 Hours. ANL Neg. No. 190-887. 


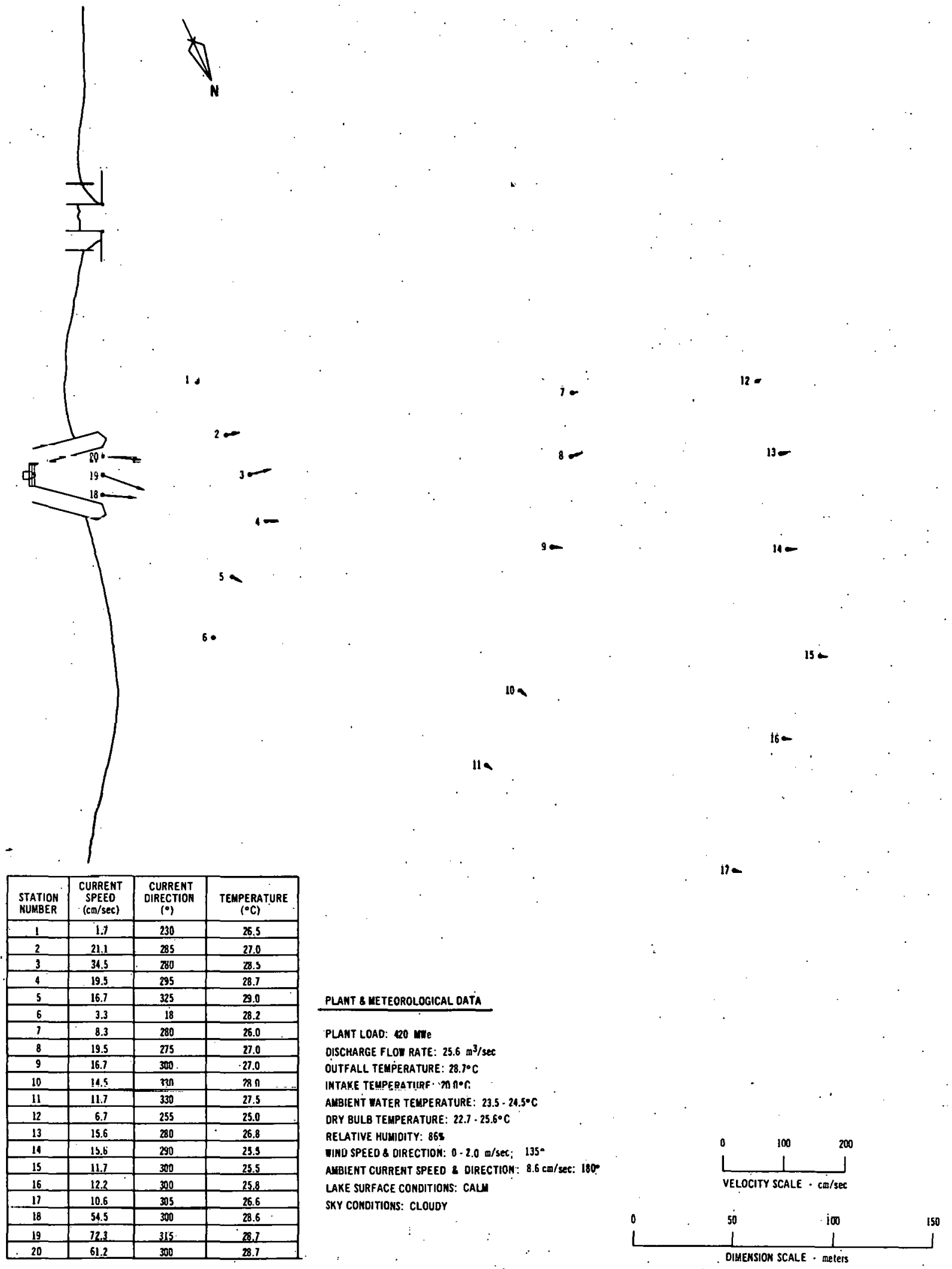

Fig. 37. Jet-regime Study for $0.5-\mathrm{m}$ Depth at Palisades Power Plant: July 19, 1972, 0922-1414 Hours. ANL Neg. No. 190-758 Rev. 1. 


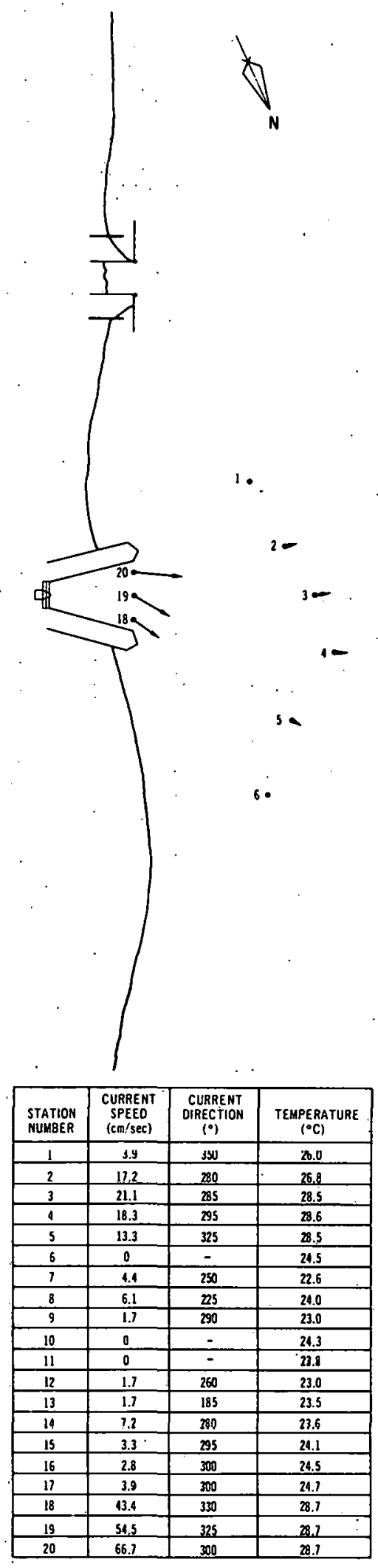

PLANT \& METEOROLOGICÁL DATA.

PLANT LOAD: 420 MIY

OISCHARGE FLOW RATE: $25.6 \mathrm{~m}^{3 / 3} / \mathrm{sec}$

OUTFALL TEMPERATURE: $238.7^{\circ} \mathrm{C}$

INTAKE TEMPERATURE: $20.0^{\circ} \mathrm{C}$

AMBIENT WATER TEMPERATURE: $22.1 \cdot 23.7 \cdot \mathrm{C}$

DRY BULB TEMPERATURE: $22.7 \cdot 25.6^{\circ} \mathrm{C}$

RELATIVE NUMIOITY: $86 \%$

WINO STECO O DIRCOTION: $0.2 .0 \mathrm{a} / 3 \mathrm{3C:}$ J JJ"

AMBBIENT CURRENT SPEEO \& DIRECTION: $8.6 \mathrm{~cm} / \mathrm{sec}$; $180^{\circ}$

LAKE SURFACE CONOITIONS: CALM

12 .

13.

11.

10.

16

II•

$15 \cdot$

SKY CUMUUI IONS: GLOUUY

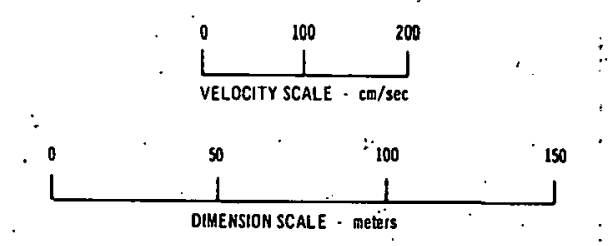

Fig. 38. "Jet-regime Study lor:1.0-ini Depth at Pallsades Power Plant: July 19. 1972, 0922-1414 Hours.: ANL Neg. No. 190-873. 


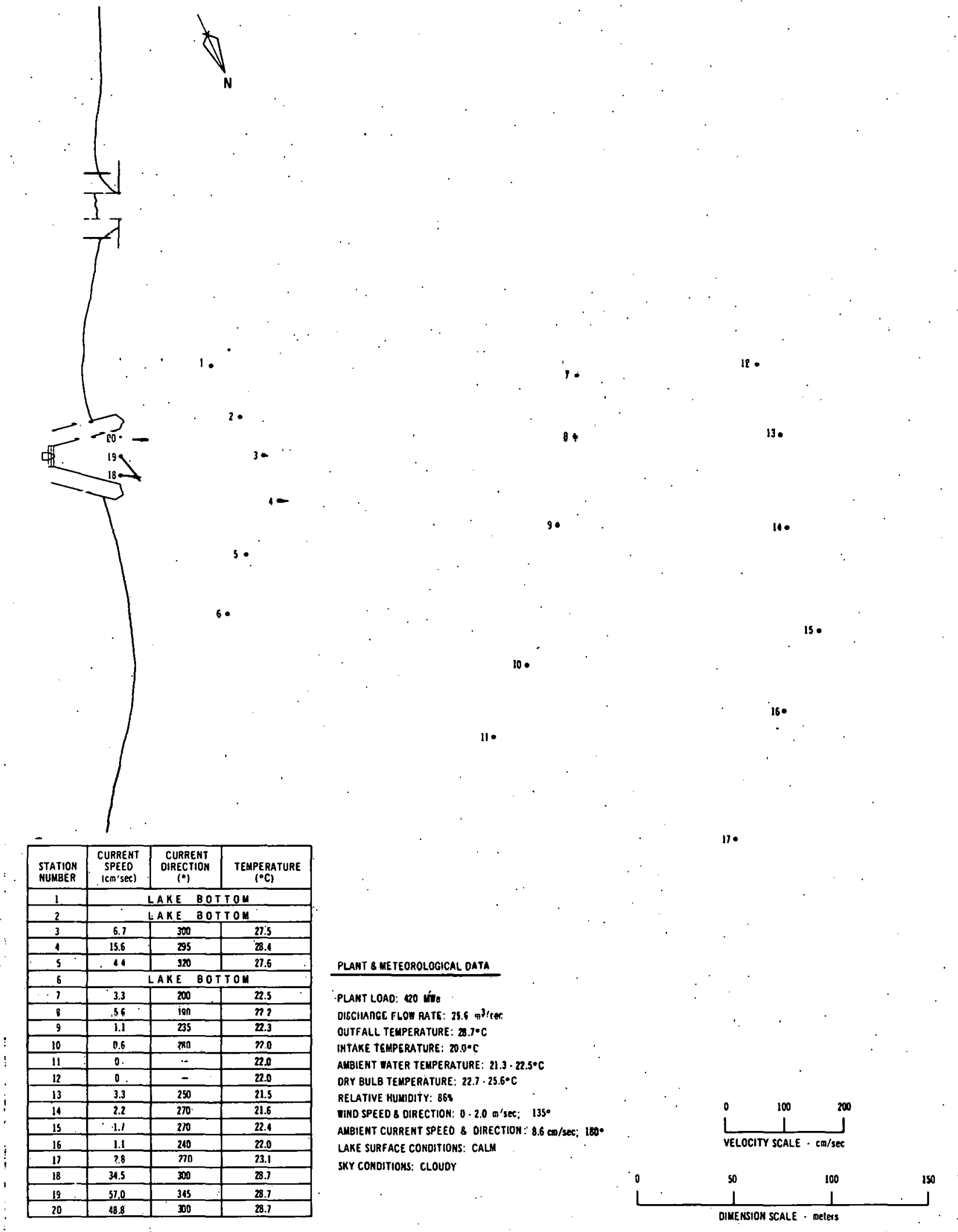

Fig. 39. Jet-regime Study for 1.5-m Depth at Palisades Power Plant: July 19, 1972, 0922-1414 Hours. ANL Neg. No. 190-884. 

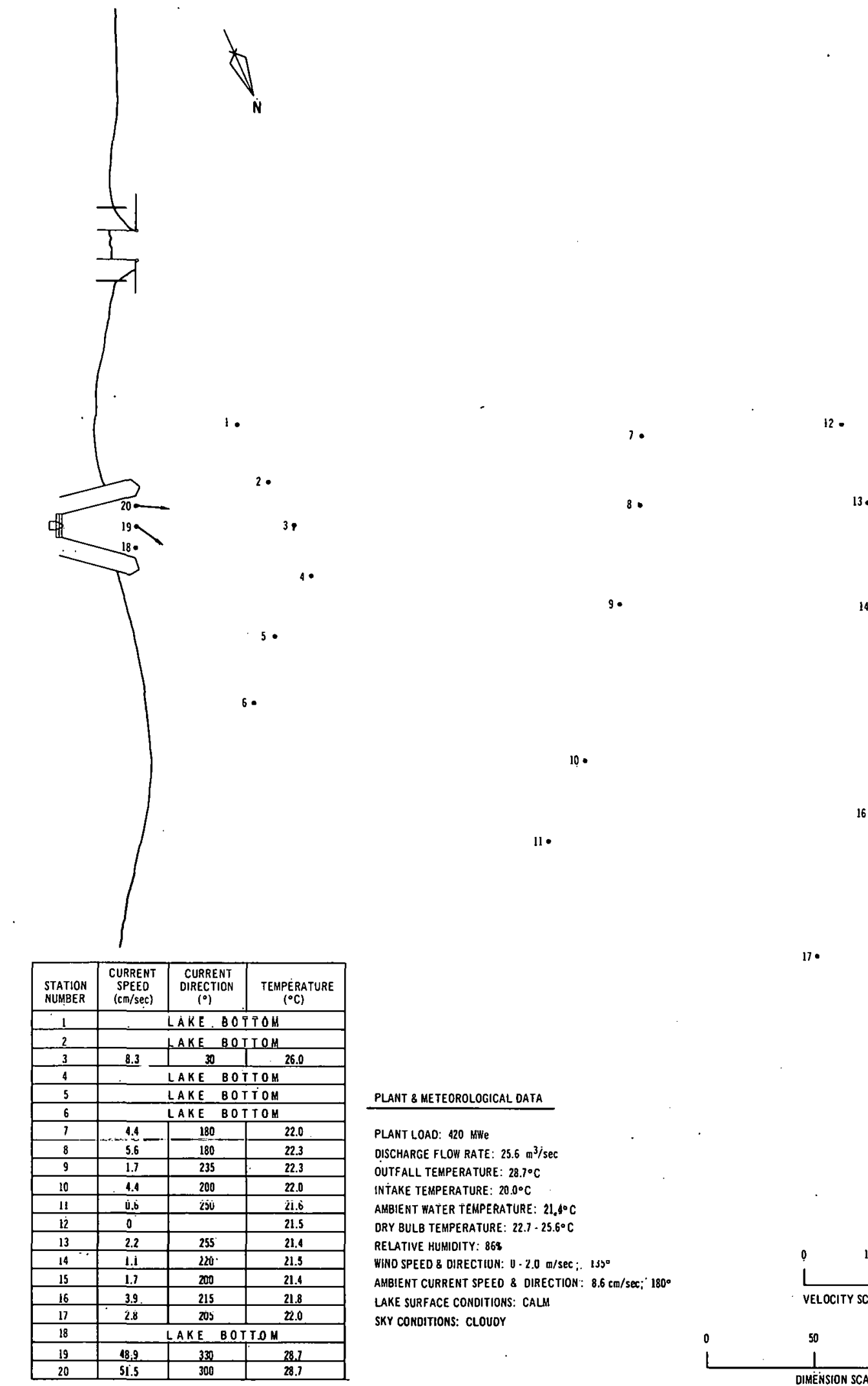

$9 \cdot$

$14 \cdot$

15 .

$10 \cdot$

16 •

$11 \bullet$

$17 \cdot$

PLANT \& METEOROLOGICAL DATA

PLANT LOAD: 420 MWE

OISCHARGE FLOW RATE: $25.6 \mathrm{~m}^{3} / \mathrm{sec}$

OUTFALL TEMPERATURE: $28.7^{\circ} \mathrm{C}$

INTAKE TEMPERATURE: $20.0^{\circ} \mathrm{C}$

AMBEENT WATER TEMPERATURE: 21.40

DRY BULB TEMPERATURE: 22.1 - 25.6. C

RELATIVE HUMIOITY: $86 \%$

WIND SPEED \& DIRECIIUN: $0 \cdot 2.0 \mathrm{~m} / \mathrm{sec}$;. $135^{\circ}$

AMBIENT CURRENT SPEED \& DIRECTION: $8.6 \mathrm{~cm} / \mathrm{sec} ;{ }^{\circ} 180^{\circ}$ LAKE SURFACE CONOITIONS: CALM

SKY CONDITIONS: CLOUOY

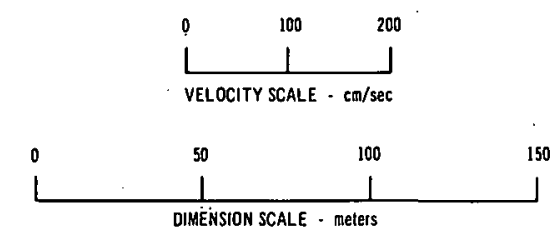

Fig. 40. Jel-reginte Study for 2.0-m Deprh ar. Pallsades Power Plant: July 19, 1972, 0922-1414 Hours. ANL Neg. No. 190-871 Rev. 1. 


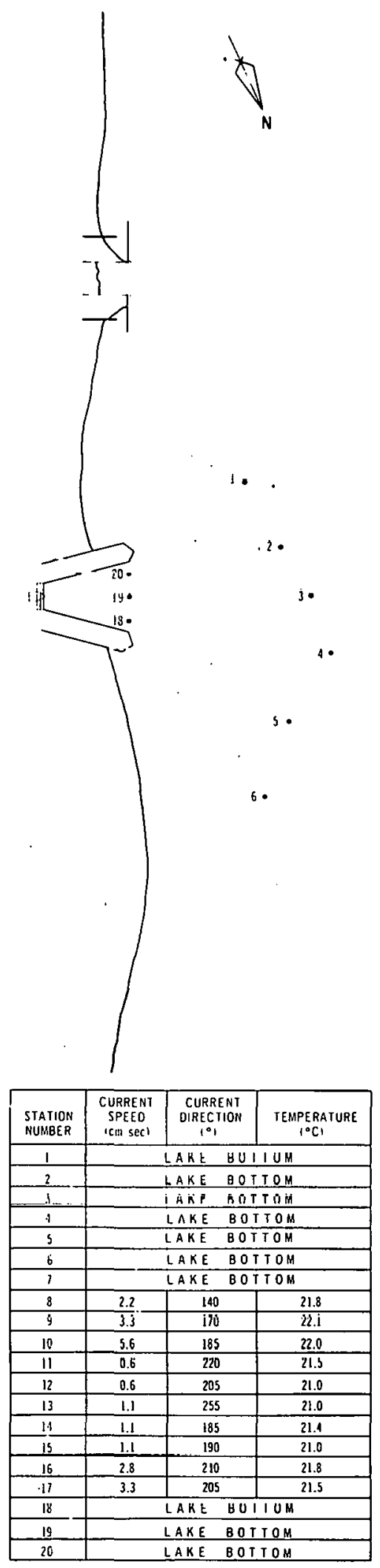

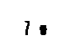

8.

9 -

14.

10.

II •

\section{PLANT LOAO: 420 MWE}

DISCHARGE FLOW RATE. $25.6 \mathrm{~m}^{3} \mathrm{sec}$

OUTFALL TEMPERATURE: $28.7^{\circ} \mathrm{C}$

INTAKE TEMPERATURE. $20.0^{\circ} \mathrm{C}$

AMBIENT WATER IEMPERATURE $21.0^{\circ} \mathrm{C}$

ORY BULB TEMPERATURE $227.256^{\circ} \mathrm{C}$

RELATIVE HUMIDITY: $85^{\circ}$.

WIND SPEED $\&$ DIRECTION. $0.20 \mathrm{~m} \mathrm{sEC;} 135 \circ$

AMBIENT CURRENT SPEED \& DIRECTION. $5.0 \mathrm{~cm} / \mathrm{sec}$ : $200^{\circ}$

LAKE SURFACE CONDITIONS: CALM

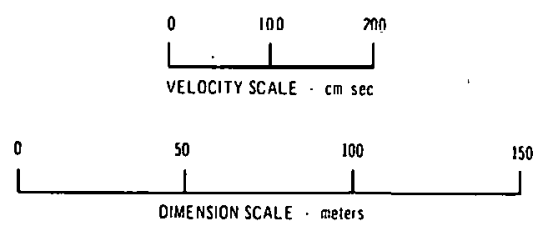

SKY CONDITIONS: CLOUOY

Fig. 41. Jet-regime Study for 2.5-m Depth at Palisades Power Plant:

July 19, 1972, 0922-1414 Hours. ANL Neg. No. 190-881. 


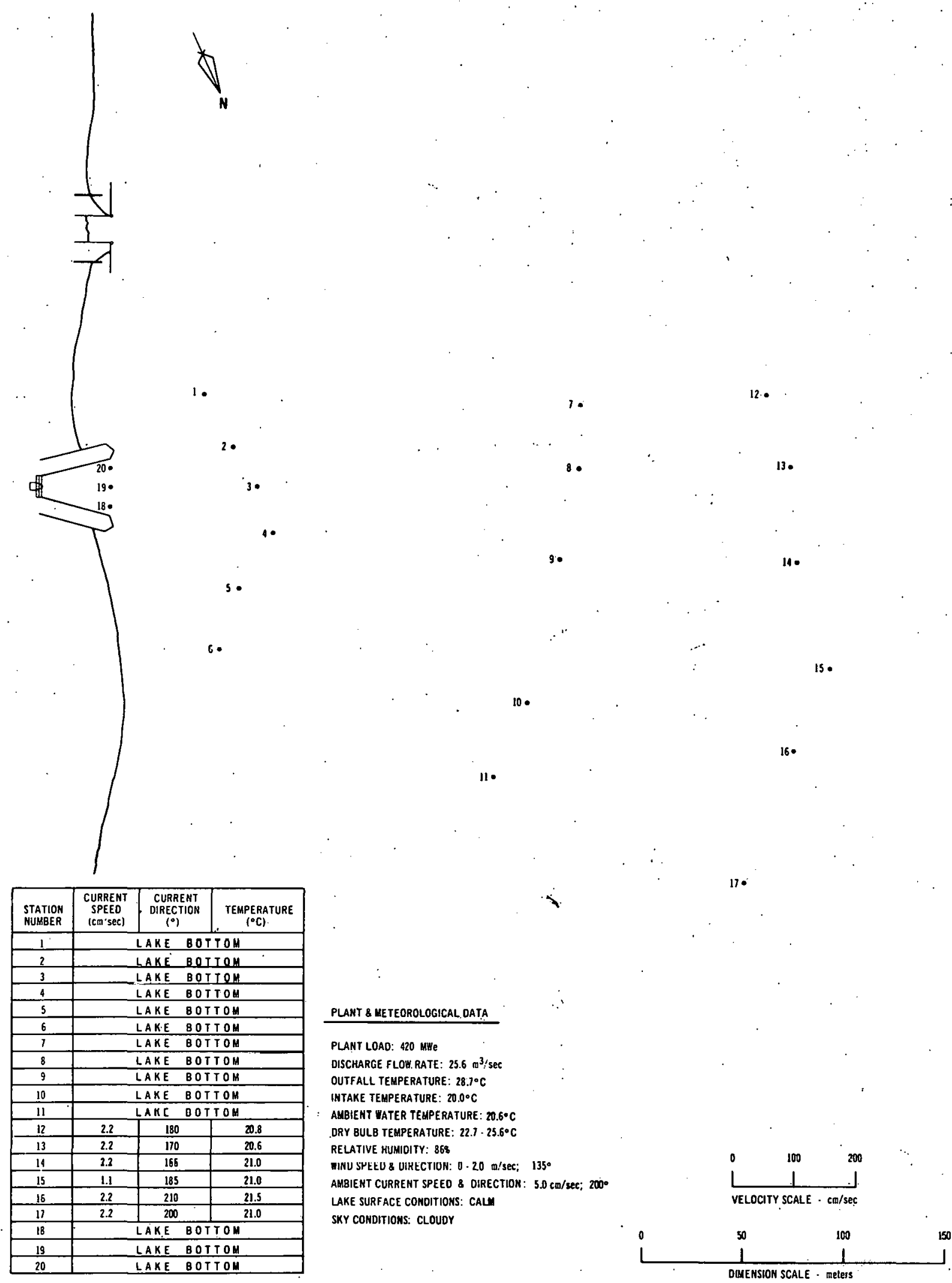

Fig. 42. Jet-regime Study for 3.0-m Depth at Palisades Power Plant: July 19, 1972, 0922-1414 Hours. ANL Neg. No. 190-882 Rev. 1. 


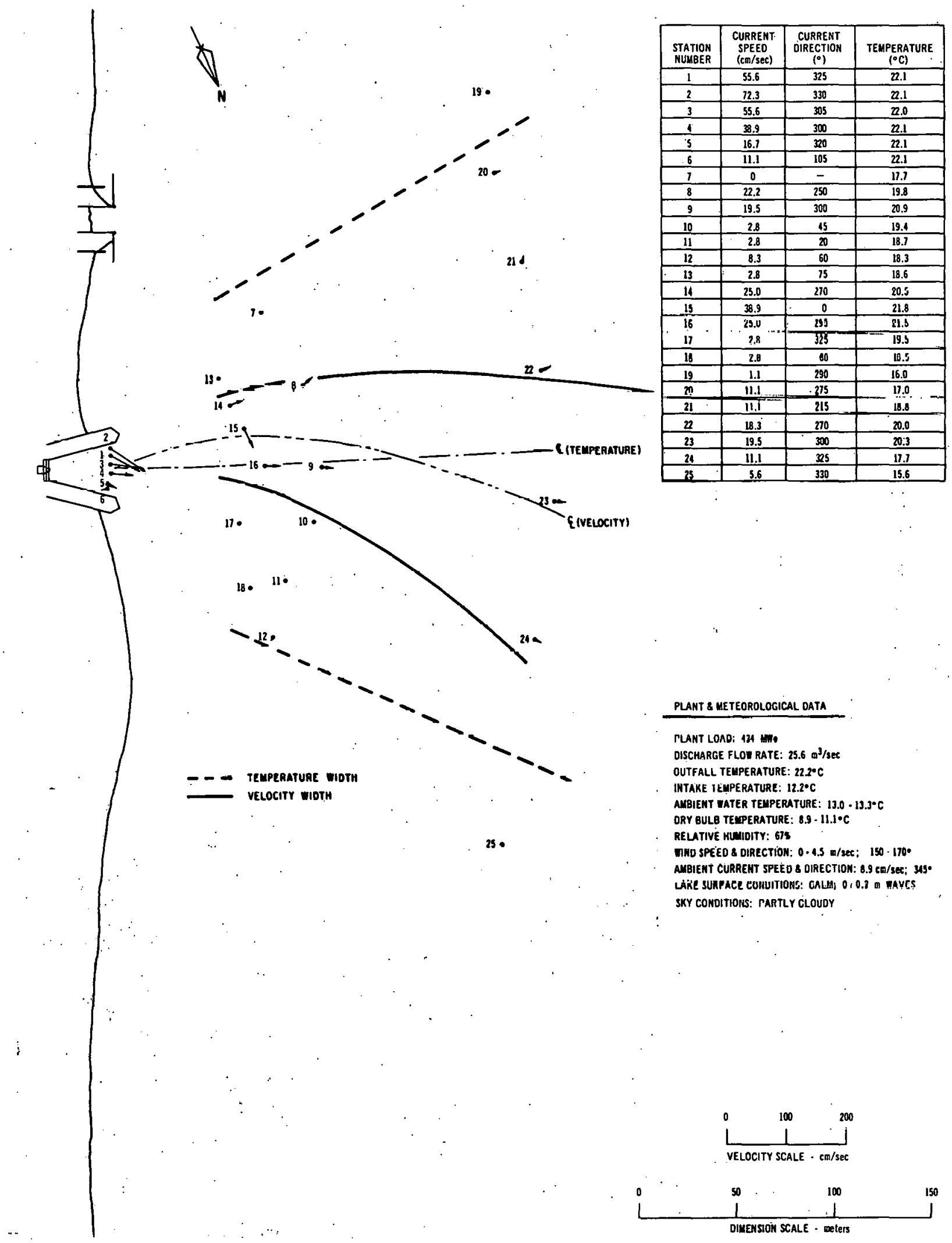

Fig. 43. Jet-regime Study for $0.5-\mathrm{m}$ Depth at Palisades Power Plant: October 10, 1972, 1025-1550 Hours. ANL Neg. No. 190-759 Rev. 1 . 


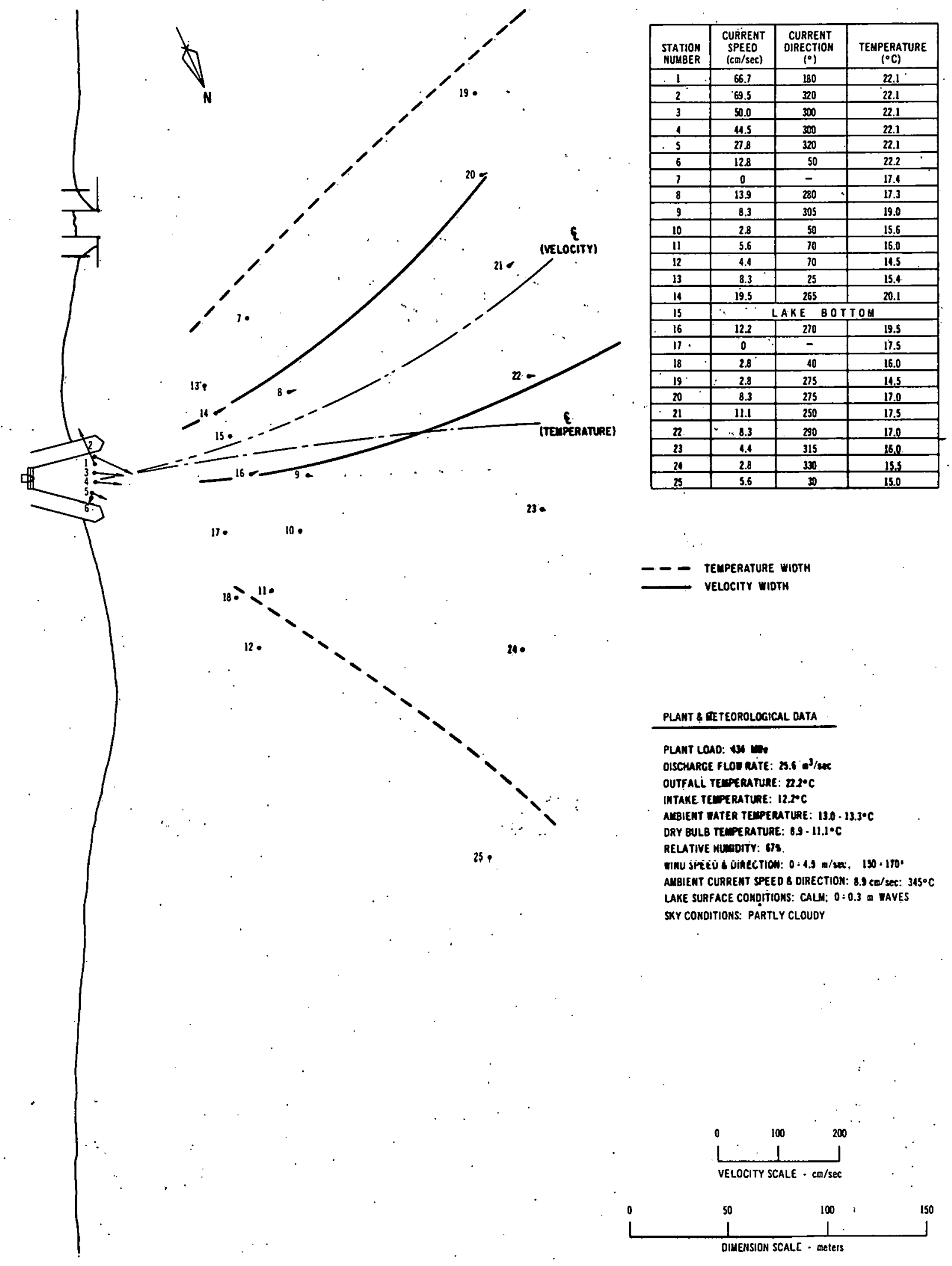

Fig. 44. Jet-regime Study for $\ddot{100-m}$ Depth at Palisades Power Plant: October 10, 1972, 1025-1550 Hours. ANL Neg. No. 190-883. 


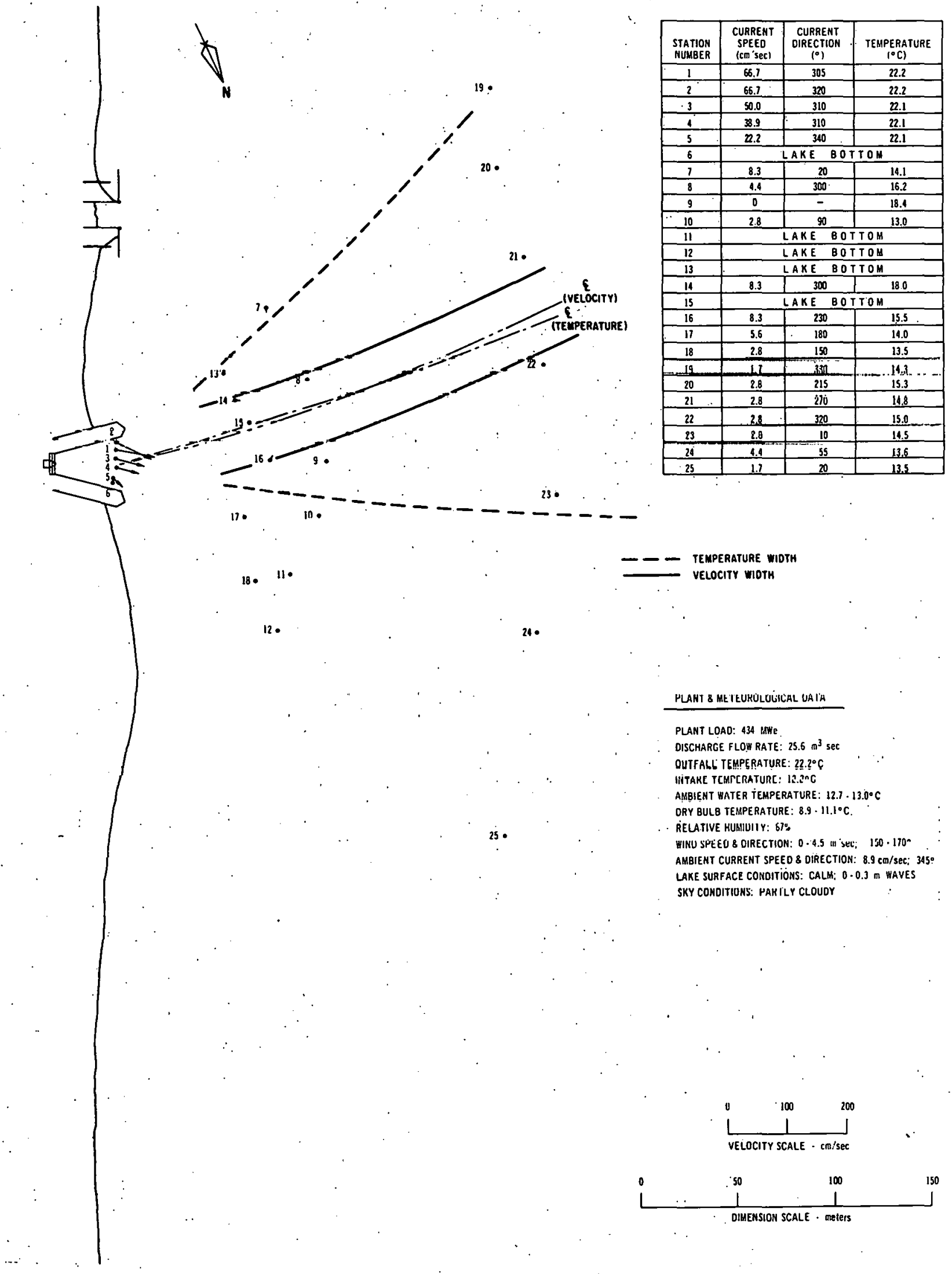

Fig. 45. Jet-regime Study for $1.5-\mathrm{m}$ Depth at Palisades Power Plant: October 10, 1972, 1025-1550 Hours. ANL Neg. No. 190-901. 

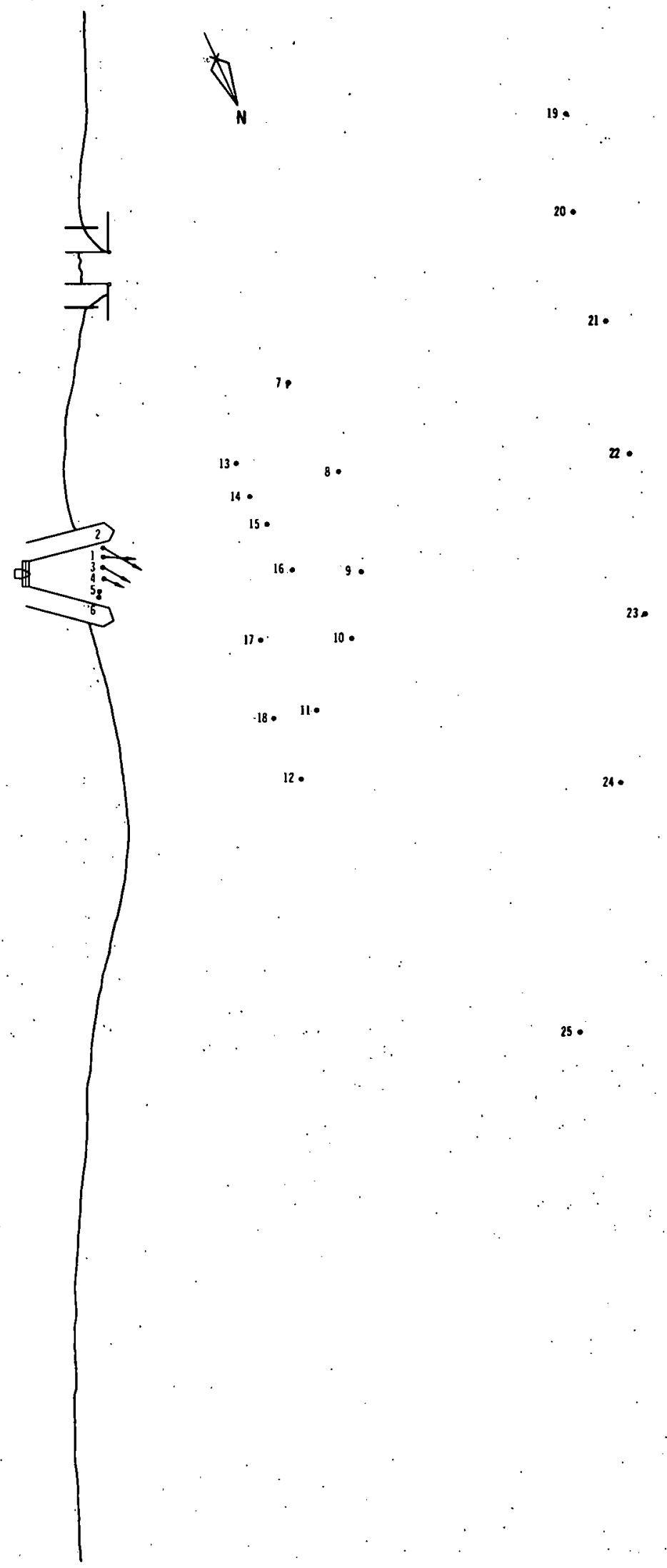

\begin{tabular}{|c|c|c|c|}
\hline $\begin{array}{l}\text { STATION } \\
\text { NUABEEA }\end{array}$ & $\begin{array}{l}\text { CURRENT } \\
\text { SPEED } \\
\text { (cm;'sec) }\end{array}$ & $\begin{array}{c}\text { CURRENT } \\
\text { OIRECTION } \\
\left.()^{\circ}\right)\end{array}$ & $\begin{array}{l}\text { TEMPERATURE } \\
\left({ }^{\circ} \mathrm{C}\right)\end{array}$ \\
\hline 1 & 44.5 & 300 & 22.2 \\
\hline 2 & 61.2 & 325 & 22.2 \\
\hline 3 & 44.5 & 325 & 22.1 \\
\hline 4 & 30.6 & 320. & 2.1 \\
\hline 5 & \multicolumn{3}{|c|}{ LAKE BOTTOM } \\
\hline 6 & \multicolumn{3}{|c|}{ LAKE BOTTOM } \\
\hline 7 & 8.3 & 40 & 13.3 \\
\hline 8 & \multicolumn{3}{|c|}{ LA.KE BOTTOM } \\
\hline 9 & \multicolumn{3}{|c|}{ LAKE BOTTOM } \\
\hline $10 .-$ & \multicolumn{3}{|c|}{ LAKE BOTTOM } \\
\hline 11 & \multicolumn{3}{|c|}{ LAKE BOTTOM } \\
\hline 12 & \multicolumn{3}{|c|}{ LAKE BOTTOM } \\
\hline 13 & \multicolumn{3}{|c|}{ LAKE $\quad$ BOTTOM } \\
\hline 14 & \multicolumn{3}{|c|}{ LAKE $80 T T O M$} \\
\hline 15 & \multicolumn{3}{|c|}{ LAKE BOTTOH } \\
\hline 16 & \multicolumn{3}{|c|}{ LAKE $80 T T O H$} \\
\hline 17 & \multicolumn{3}{|c|}{ LAKE BOTTOM } \\
\hline 18 & \multicolumn{3}{|c|}{ LAKE BOTTOM } \\
\hline 19 & 5.6 & 345 & 13.1 \\
\hline 20. & 1.1 & 325 & 13.7 \\
\hline 21 & 2.8 & 305 & 13.4 \\
\hline 22 & 4.4 & 40 & 14.0 \\
\hline 23 & 6.7 . & 80 & 14.3 \\
\hline 24 & 4.1. & 70 & 13.1 \\
\hline 25 & 2.8 & 75 & 13,1 \\
\hline
\end{tabular}

17.10.

23

18. $11 \cdot$

$12 \cdot$

$24 \cdot$

25 .

PLANT \& METEOROLOGICAL DATA

PLANT LOAD: 434 MHE

OISCHARGE FLOI RATE: $25.6 \mathrm{im}^{3} / \mathrm{sec}$

OUTFALL TEMPERATURE: $22.2^{\circ} \mathrm{C}$

INTAKE TEMPERATURE: $12.2^{\circ} \mathrm{C}$

AMBIENT WATER T.EMPERATURE: $12.8 \cdot 13.0^{\circ} \mathrm{C}$

DRY BULB TEMPERATURE: $8.9 \cdot 11.1{ }^{\circ} \mathrm{C}$

RELATIVE HUMIOIY: $67 \%$

WIND SPEED 8 OIRECTION: $0-4.5 \mathrm{~m} / \mathrm{sec}$; $150 \cdot 170^{\circ}$

AMBIENT CURRENT. SPEE D 8 DIRECTION: $8.9 \mathrm{~cm} / \mathrm{sec} ; \cdot 345^{\circ}$

LAKE SURFACE CONDITIONS: CALM; 0.0 .3 a vaVES

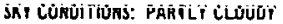

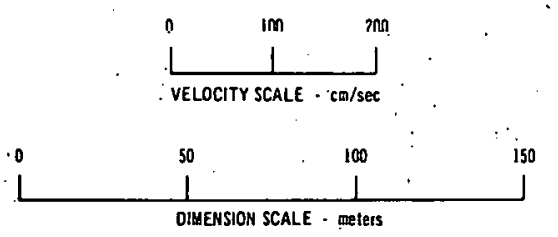

Fig. 46. Jet-regime study for $: 2.0-m$ Depth at Palisades Power Plant:

October 10, 1972, 1025-1.550 Hours. ANI. Neg. No. 190-876. 


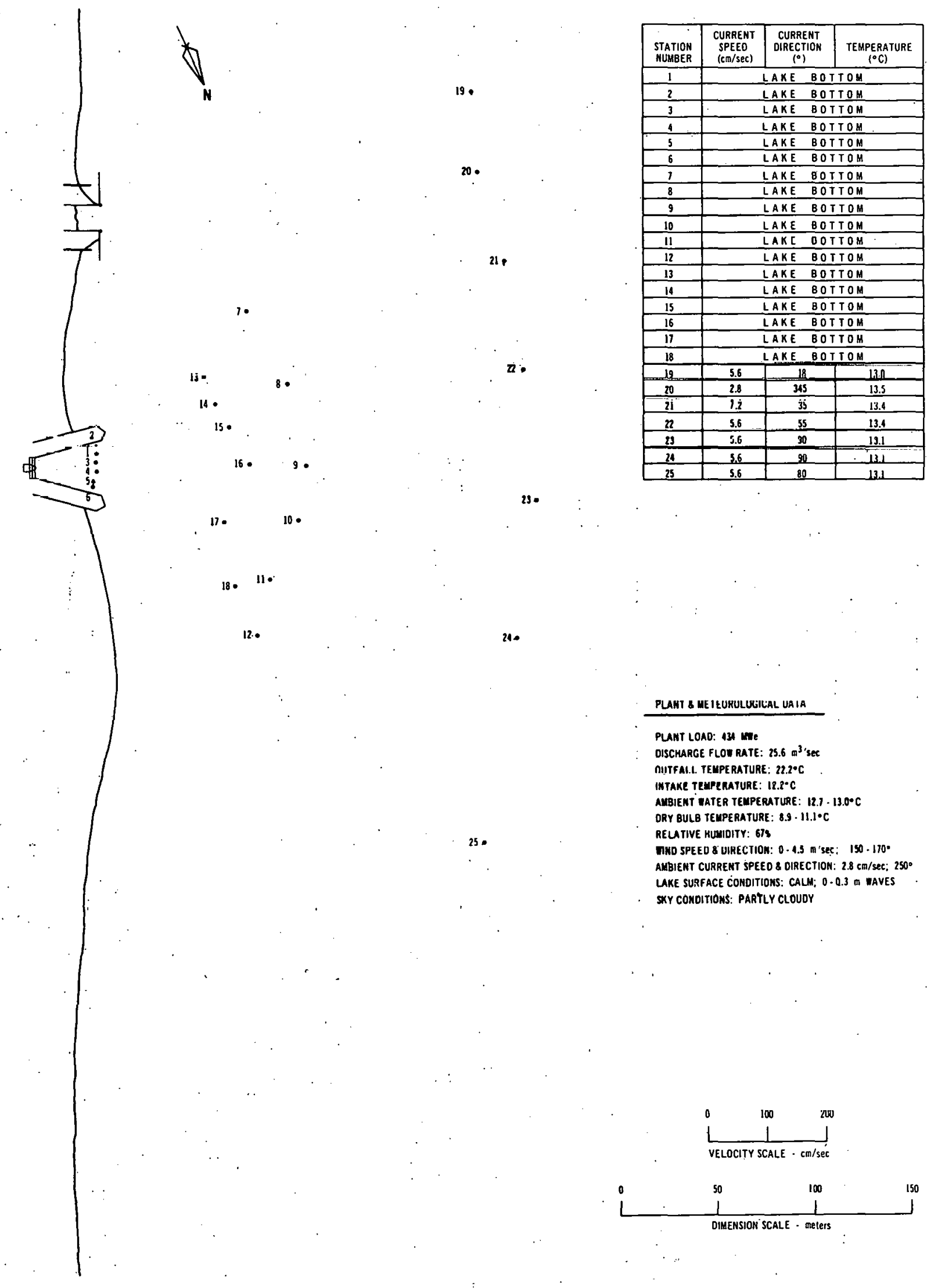

Fig. 47. Jet-regime Study for $2.5-\mathrm{m}$. Depth at Palisades Power Plant: October 10, 1972, 1025-1550 Hours. ANL Neg: No. 190-886. 


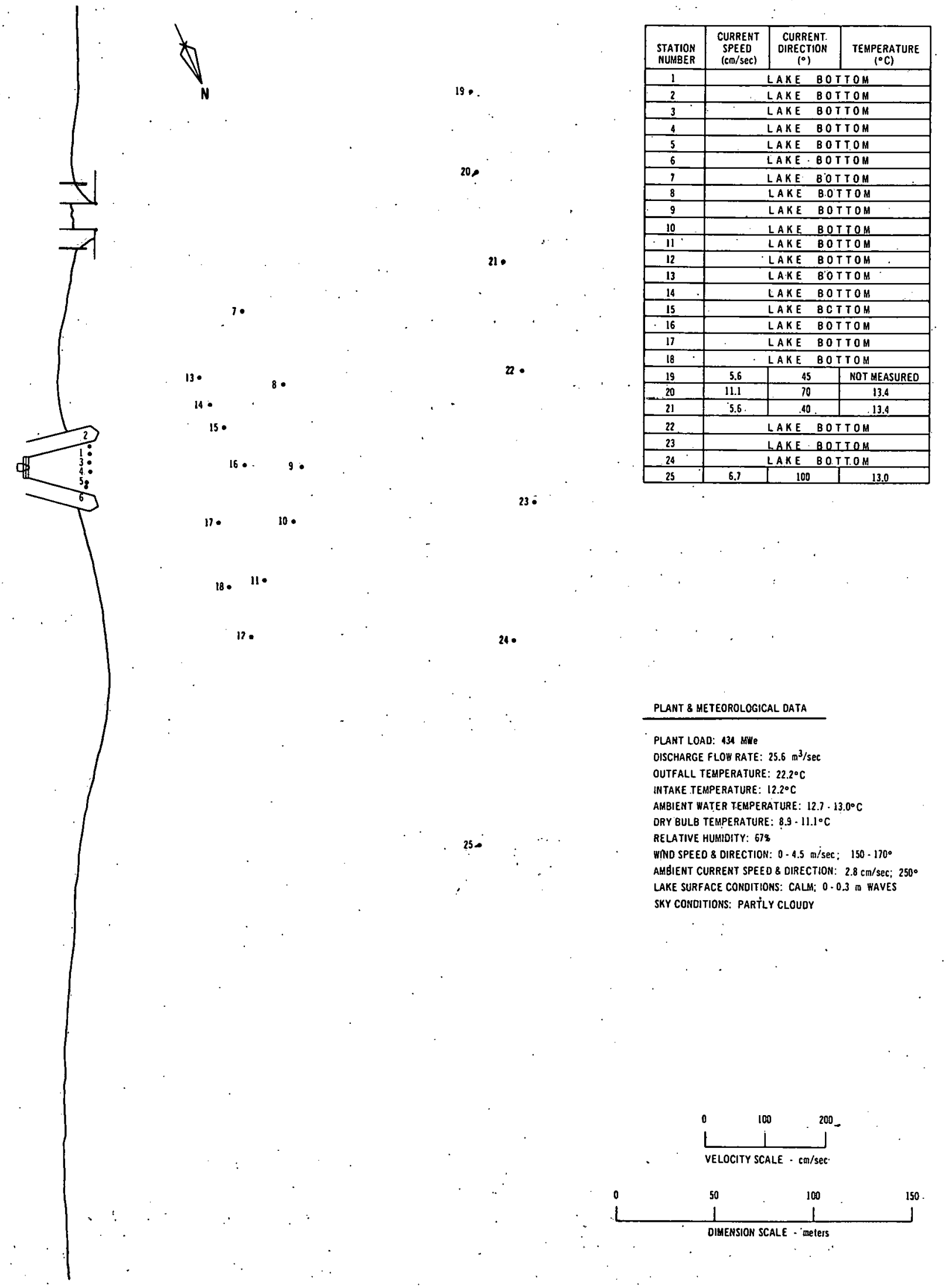

Fig. 48. Jet-regime Study for $3.0-\mathrm{m}$ Depth at Palisades Power Plant: October 10, 1972, 1025-1550 Hours. ANL Neg. No. 190-874. 


\section{EXTRACTION OF JET CHARACTERISTICS FROM FIELD MEASUREMENTS}

In order to examine the features of the temperature and velocity dis tributions in the near-field region of the discharges, and additionally to facilitate comparisons with analytical-model predictions, it was decided to assume a functional form for the distributions and fit these distributions to the field measurements. The choice of the functional forms was based on preliminary examination of the Point Beach data. The functions chosen use Gaussian dis tributions for the lateral excess-temperature and excess-velocity profiles. The excess temperature $\theta$ is defined as the difference between the measured temperature $T$ and the ambient temperature $T_{A}$ of the lake water at that depth. The excess velocity $u_{E}$ is defined as the component of the measured velocity parallel to the centerline of the jet, minus the component of the ambient current parallel to the centerline. The ambient current $u_{A}$ was taken to be parallel to shore for this purpose. The widths of these profiles are assumed to'grow linearly with distance from lise putfall, 0, as measured along the jet centerline. The centerline temperature excess $\theta_{c}$ and the centerline velocity excess $u_{c E}$ are assumed to decay as the inverse of the square root of the distance from the outfall. A quadratic form was chosen for the trajectories of the centerline.

The temperature excess at a lateral distance $\eta$ from the temperature centerline at a distance $s$ from the outfall is thus given by

$$
\theta(\eta, s)=\theta_{c}(s) \exp \left\{-(\ln 2)\left[\eta / w_{T}(s)\right]^{2}\right\}=\theta_{c}(s)\left(\frac{1}{2}\right)^{\left[\eta / W_{T}(s)\right]^{2}},
$$

where

$$
\theta_{c}(s)= \begin{cases}\theta_{0} A & \text { for } s \leq \frac{\alpha b_{0}}{A^{2}} \\ \theta_{0} \sqrt{\frac{\alpha \mathrm{b}_{0}}{s}} & \text { for } s>\frac{\alpha b_{0}}{A^{2}}\end{cases}
$$

and

$$
\mathrm{W}_{\mathrm{T}}(\mathrm{s})=\mathrm{Cb} \mathrm{b}_{\mathrm{O}}+\gamma \mathrm{s}
$$

In the above expression, $\theta_{0}$ is the initial temperature excess at the outfall, $b_{0}$ is the width of the outfall, and $W_{T}$ is the half-width of the excess temperature distribution. The half-width is defined as the lateral distance at which the excess temperature drops off to one-half the centerline value. The dimensionless pärameters $A, \alpha, C$, and $\gamma$ are free parameters to be determined by fitting to the field data. 
To specify the centerline, a coordinate system is set up with the origin at the center of the outfall. The positive $y$ axis is perpendicular to the average shoreline in the off-shore direction, the positive $\mathrm{z}$ axis is vertically upward, and the positive $\mathrm{x}$ axis is chosen to be orthogonal to the other two so as to form a right-handed system in the conventional sense $(x, y, z)$. The temperature centerline trajectory is most easily specified in parametric form:

$$
\mathrm{x}_{\mathrm{CT}}=\xi \cos \left(\mathrm{R}_{\mathrm{T}} \beta_{0}\right)-\frac{\mathrm{K}_{\mathrm{T}}}{100 \mathrm{~b}_{0}} \xi^{2} \sin \left(\mathrm{R}_{\mathrm{T}} \beta_{0}\right)
$$

and

$$
y_{\mathrm{CT}^{\circ}}=\xi \sin \left(\mathrm{R}_{\mathrm{T}} \hat{\beta}_{0}\right)+\frac{\mathrm{K}_{\mathrm{T}}}{100 \mathrm{~b}_{0}} \xi^{2} \cos \left(\mathrm{R}_{\mathrm{T}} \beta_{0}\right)
$$

for

$$
\xi \geq 0 \text {. }
$$

The trajectory represents a parabola passing through the origin at an angle of $R_{T} \beta_{0}$ with respect to the positive $x$ axis. The parametric variable $\xi$ is introduced only to simplify the form of these equations. The angle of the outfall with respect to the positive $x$ axis is $\beta_{0}$, and $R_{T}$ and $K_{T}$ are two dimensionless free parameters to be determined by fitting to the field data.

The case for which $R_{T}=1.0$ and $K_{T}=0.0$ corresponds to a jet centerline that is directed straight out from the outfall. Positive values. of $\mathrm{K}_{\mathrm{T}}$ correspond to the jet bending to the left, and negative values correspond to bending to the right. The quantity $R_{T} \beta_{0}$ determines the initial angle of the centerline at the origin (discharge).

The velocity excess (assumed parallel to the centerline) at a lateral distance $\eta$ from the velocity centerline is given by the following expression:

$$
u_{E}(\eta, s)=u_{C E}(s)\left(\frac{1}{2}\right)^{\left[\eta / w_{u}(\dot{s})\right]^{2}},
$$

where-

$$
u_{C E}(s)= \begin{cases}u_{0} R & \text { for } s \leq \frac{\beta b_{0}}{B^{2}} \\ u_{0} \sqrt{\frac{\beta b_{0}}{s}} & \text { for } s>\frac{\beta b_{0}}{B^{2}} .\end{cases}
$$

and

$$
\mathrm{W}_{\mathrm{u}}(\mathrm{s})=\mathrm{D} \mathrm{b}_{0}+\dot{\delta}_{\mathrm{s}}
$$


These velocity-excess equations have the same form as the expressions for the temperature excess, except that $u_{0}$ is the outfall velocity averaged over the entire outfall. The velocity centerline has a form analogous to that for the temperature centerline:

$$
x_{c u}=\xi \cos \left(R_{u} \beta_{0}\right)-\frac{K_{u}}{100 b_{0}} \xi^{2} \sin \left(R_{u} \beta_{0}\right)
$$

and

$$
y_{c u}=\xi \sin \left(R_{u} \beta_{0}\right)+\frac{K_{u}}{100 b_{0}} \xi^{2} \cos \left(R_{u} \beta_{0}\right)
$$

The parameters $B, \beta, D, \delta, R_{u}$, and $K_{u}$ are determined by fitting to the data.

The temperature and velocity functions described above, each with only six free parameters, are faily restrictive. Howuver, due to thr limited amount of data available, it was felt that more general functions with more free parameters would be unwarranted. As a consequence, only the general trends of the resulting fitted function can be considered significant. The details are artifacts of the functions chosen.

The fitting procedure was carried out on the computer by a minimization technique. The root-mean-square deviations of the functions from the data are defined as follows:

$$
\left(\sigma_{\mathrm{T}} ; \mathrm{A}, \alpha, \mathrm{C}, \gamma, \mathrm{R}_{\mathrm{T}}, \mathrm{K}_{\mathrm{T}}\right)=\left[\sum_{i=1}^{\mathrm{N}} \frac{\left(\theta_{\mathrm{Di}}-\theta_{\mathrm{Fi}}\right)^{2}}{\mathrm{~N}-6}\right]^{1 / 2}
$$

and

$$
\left(\sigma_{\mathrm{u}} \mathrm{B}, \boldsymbol{\beta}, \mathrm{D}, \delta, \mathrm{R}_{1,1}, \mathrm{~K}_{\mathrm{i} i}\right) \cdot=\left[\sum_{i=1}^{N} \frac{\left(u_{\mathrm{Di}}-u_{F i}\right)^{?}}{6}\right]^{1 / 2},
$$

where $N$ is the number of data points, $\theta_{D i}$ is the measured temperature excess at the ith data point, $\theta_{F i}$ is the temperature excess calculated from the function, $u_{\mathrm{Di}}$ is the component of the measured velocity excuss parallel. to the velocity centerline, and $u_{F i}$ is the velocity excess calculated from the function. The 6 in the denominator is introduced to account for the six degrees of freedom associated with the six free parameters of the fitting function. The final values of the parametcre were then shosen to be those values that minimize $\sigma_{\mathrm{T}}$ and $\sigma_{\mathrm{u}}$. The FORTRAN program JETFIT used for this fitting procedure is listed in Appendix C.

The sets of measurements taken during the four jet studies at the Point Beach outfall and one of the studies at the Palisades outfall (October 10, 1972) were each fitted independently. The measurements of the 
other two Palisades surveys did not lend themselves to this fitting procedure because the limited number of data points did not appear to characterize a major portion of the jet region; i.e., the jet seems to be significantly wider than the region surveyed.

The parameters resulting from the fitting procedure and a tabulation of the deviations of the fitted functions from the measurements are included in Appendix D. The average deviation of the function from the data varied from about 0.2 to $1.1 \mathrm{C}^{\circ}$, with an overall average deviation for all five studies of $0.7 \mathrm{C}^{\circ}$. The average velocity deviation varied from 1 to $8 \mathrm{~cm} / \mathrm{sec}$, with an overall average deviation of $4 \mathrm{~cm} / \mathrm{sec}$. These overall average deviations correspond to about $7 \%$ of the average initial temperature excess and outfall velocity, respectively. Figures $8-48$ show the temperature and velocity centerlines and the temperature and velocity half-widths resulting from this fitting proccdure, along with the actual field measurements. Note that the field measurements were made in groups corresponding approximately to perpendicular transects of the jet. Some of the fitting results for the Point Beach studies are shown together in Figs. 49-52 so that they may be compared with each other. The circles on the curves of $\theta_{c} / \theta_{0}$, plotted as a function of distance from the outfalls (Fig. 49), indicate the approximate location of the measurement groupings. The results for the one Palisades case are presented in Sec. VIl along with the model comparisons.

Figure 49 shows the centerline temperature and velocity decays resulting from the fits to the measurements taken at a depth of $0.5 \mathrm{~m}$ for all four Point Beach dates. The drop-off rates are approximately the same for each survey and for both temperature and velocity, except for the velocity results of July 13, 1972. This difference is presently unexplained. Indeed, the differences shown in the figures, with that one exception, are probably within the errors associated with the experimental measurements and the fitting procedure. On all four dates, the power plant was operating at essentially the same power level and discharge flow rate. The temperature dis tribution across the outfall has been measured and found to be quite uniform. Therefore, the excess temperature ratio $\theta_{c} / \theta_{0}$ plotted in the upper half of Fig. 19 must be 1.0 at $s=0$. This restriction was not placed on the fitting function, and so not much significance should be attached to the details contained in the first $100 \mathrm{~m}$ of the fitting results. The initial velocity distribution at the Point Beach outfall has also been measured.*. 'It was not found to be uniform. In fact, velocities as much as $60 \%$ above the average outfall velocity were observed. This fact shows up in the lower half of Fig. 49, where the velocity ratio exceeds 1.0 for small values of s. Again, not much significance should be attached to the details of the results in this initial $100-\mathrm{m}$ region.

Figure 50 presents the corresponding temperature and velocity halfwidths for the same four dates. It is evident that the temperature distribution is wider than the velocity distribution. 'Indeed, on the average, it is; approximately twice as wide.

*A typical outfall velncity distribution for Point Beach appears in ANL/ES-16 (RER. 19 of Appendix A). Measurements on several other dates exist and will be published in the future. 


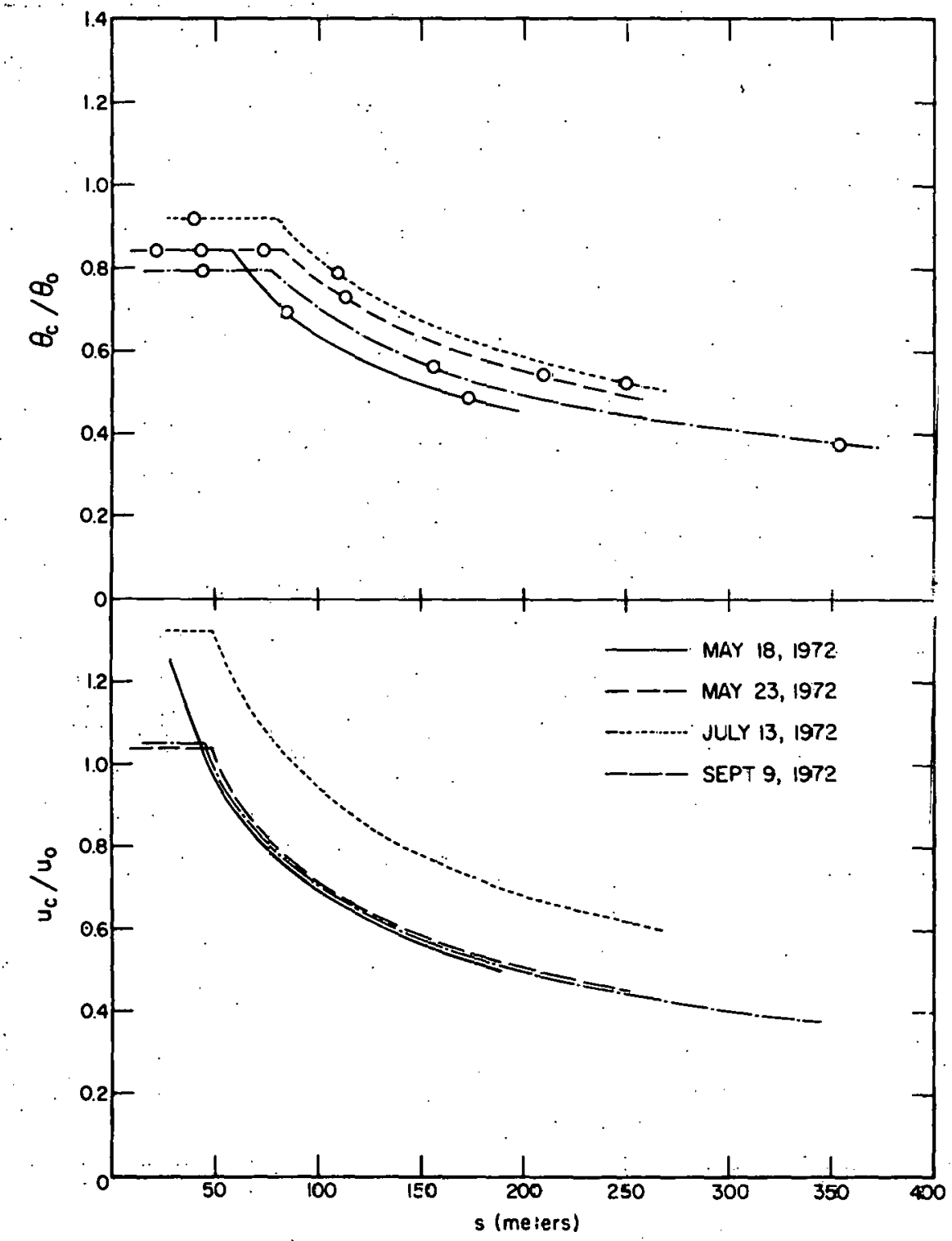

Fig. 49. Centerline Excess-temperature and -velocity Decays Resulting from Fits to Point Beach Jet Data at 0.5-m Depth. ANiL Neg. No. 196-95C.

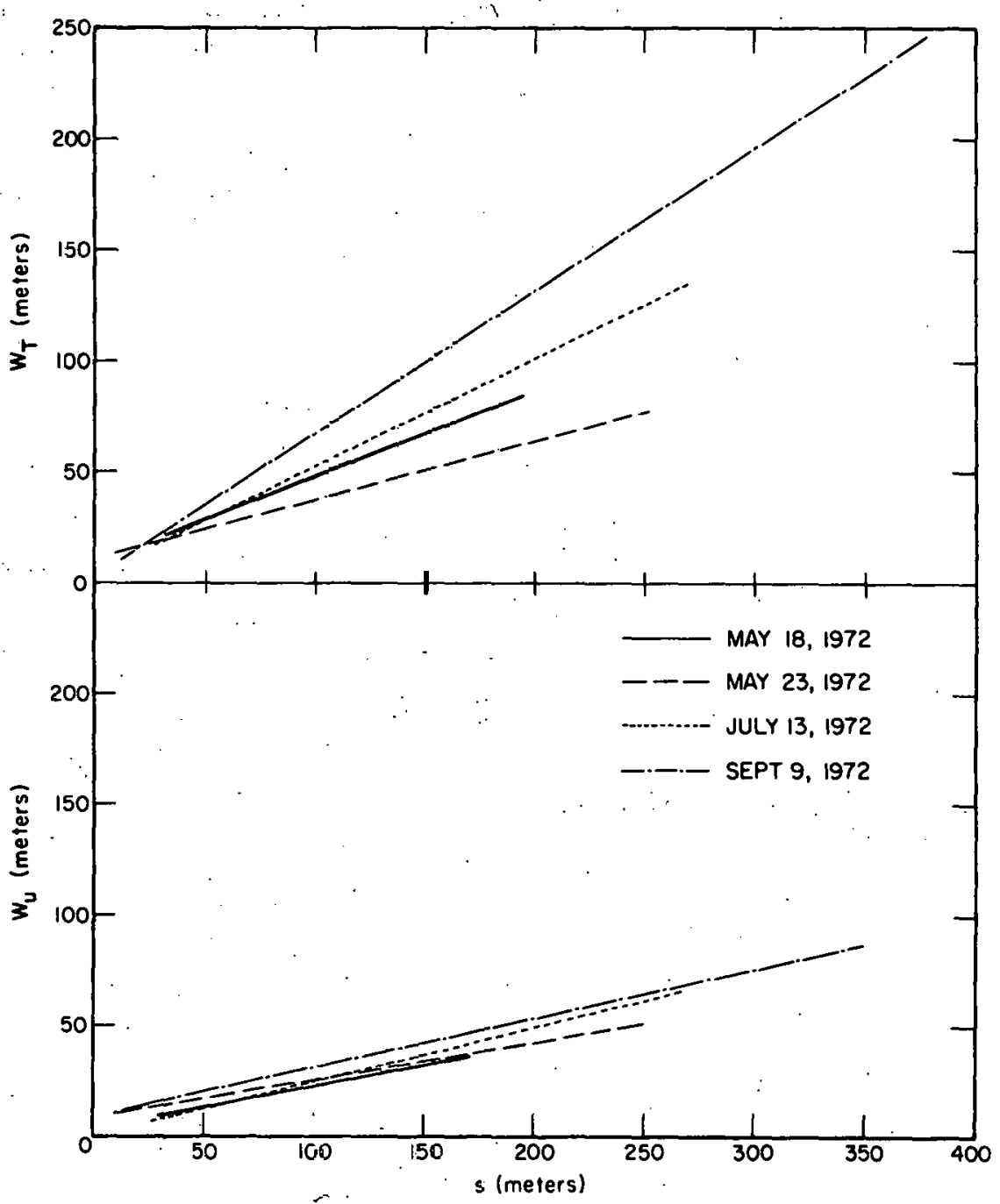

Fig. 50. Half-widths of Temperature and Velocity Distributions Resulting from Fits to Point Beach Jet Data at 0.5-m Depth. ANL Neg. No. 190-961. 


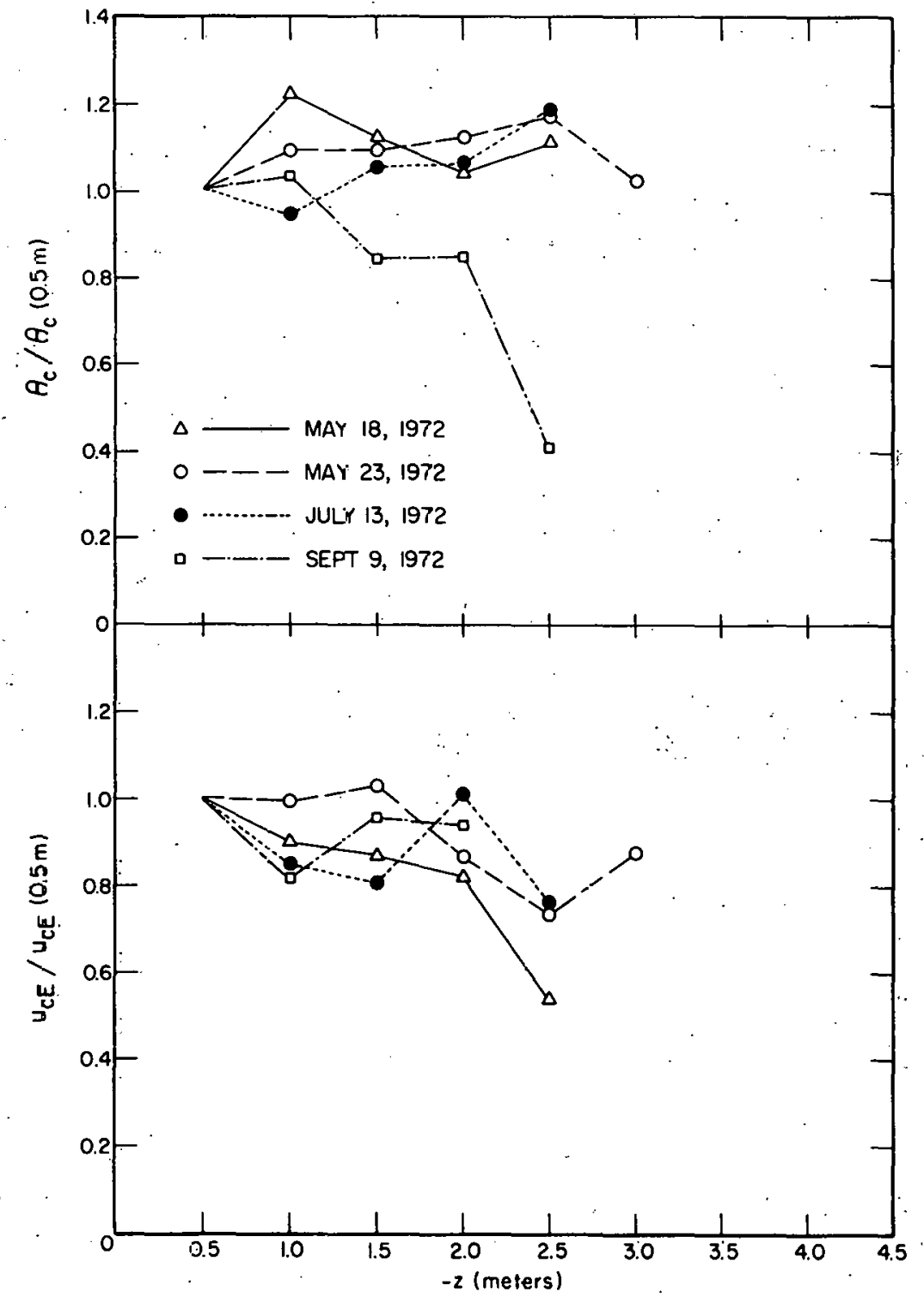

Fig. 51: Centerline Temperature Excess and Velocity Excess as a Function of Depth Resulting from Fits to Point Beach Jet Data (normalized to the $0.5-\mathrm{m}$ results). ANL Neg. No. 190-953.

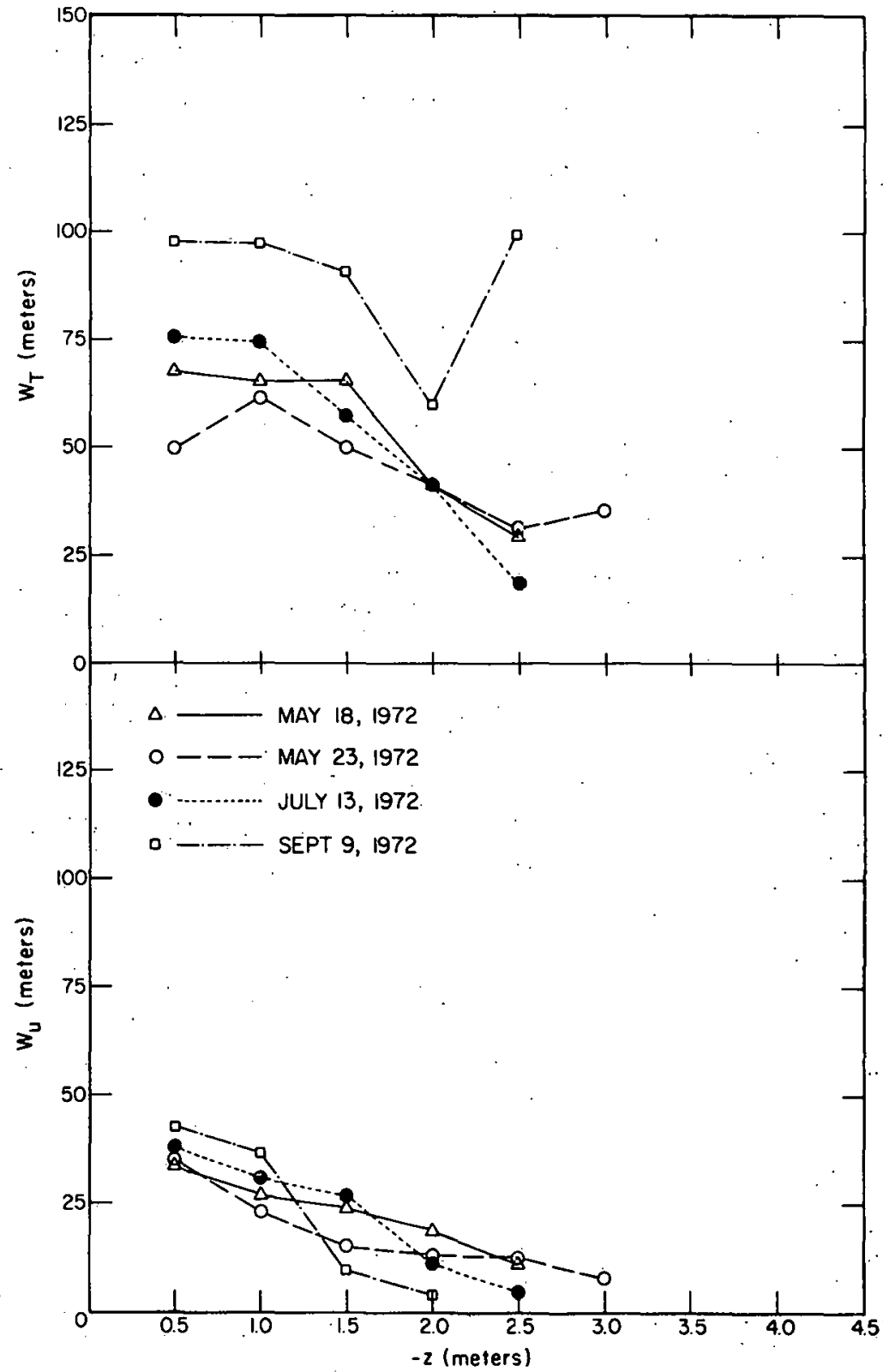

Fig. 52. Half-widths of Temperature and Velocity Distributions as a Function of Depth at $s=150 \mathrm{~m}$, Resulting from Fits to Point Beach Jet Data. . ANL Neg. No. 190-945. 
Figure 51 shows the centerline temperature and velocity excesses as a function of depth relative to the $0.5-\mathrm{m}$ results. Because of the nature of the functional form chosen, these quantities are independent of distance from the outfall after the initial region, which was always less than about $100 \mathrm{~m}$. Within the accuracy of these results, the excesses are fairly constant with depth. However, the temperature data of September 9, 1972, do show an exception. Because there is no clear drop-off with depth, a characteristic depth of the jet could not be extracted from the JETFIT results.

Figure 52 shows the widths as a function of depth at a distance of $150 \mathrm{~m}$ from the outfall. This distance was chosen because it lies approximately in the middle of the range for which measurements were made. These widths show a decreasing trend, tending toward zero at a depth of $2.5-3.0 \mathrm{~m}$. The temperature widths are approximately constant for the first $1.0-1.5 \mathrm{~m}$. and then decrease while the velocity widths decrease more uniformly.

In summary, the Point Beach jet studies indicate that the centerline excess temperature and excess velocity ratios decay at about the same rate, with jet longitudinal distance, for all four dates and for depths down to $2 \mathrm{~m}$ or more. This decay, beyond about the first $50 \mathrm{~m}$, is characterized by

$$
\frac{\theta_{c}}{\theta_{0}} \approx \frac{u_{c E}}{u_{0}} \approx \sqrt{\frac{4.2 b_{0}}{s}} .
$$

In the above expression, 4.2 is the average value of $\alpha$ and $\beta$ for all cases except for the $0.5-\mathrm{m}$ velocity results of July 13,1972 , and the 1.5-,2.0-, and 2.5-m temperature results of September 9, 1972. The average deviation of the actual values of $\alpha$ and from this ralue of 4.2 is 0.7 . 'Lhe temperature half-widths at the $0.5-\mathrm{m}$ depth grow at a rate of $3-6 \mathrm{~m}$ for each $10 \mathrm{~m}$ from the outfall; the velocity half-widths grow at only about $2 \mathrm{~m}$ per $10 \mathrm{~m}$. Both these rates decrease with depth, tending toward zero at about $2.5-3.0 \mathrm{~m}$. 


\section{MATHEMATICAL MODELING OF NEAR -FIELD REGION OF SURFACE THERMAL DISCHARGES}

The utility of analytical near-field, surface-discharge models is primarily centered around three considerations. The first of these is a desire to assess, and therefore avoid, possible recirculation of heated water into the plant intake. The second is the necessity to develop an appropriate dis charge design to satisfy temperature standards. The third is a desire to help predict possible biological effects relating to changes in the physical and chemical properties of the water.

Most presently operating power plants use a surface channel or canal to discharge their heated condenser-cooling water, and many new plants still plan for similar discharge designs. Since thermal discharges of cooling water from power plants will undoubtedly increase greatly in both magnitude and number during the next decade, the adequacy of predictive models for such discharges becomes an important factor in design.

Table II summarizes the basic characteristics of 15 near-field analytical models ${ }^{1-15}$ for surface thermal discharges presently available in the literature. Table III summarizes those complete-field models ${ }^{16-24}$ that have separate jet-regime analyses. Due to limitations of time and space, the field of 24 models had to be narrowed down considerably. Considered in this report and compared with the Point Beach Unit 1 and Palisades data are the more promising and widely used models of Pritchard (Model No. 1), Motz and Benedict, Stolzenbach and Harleman, and Prych. Some of the reasons for not including comparisons with the other models are as follows:

1. Hoopes et al.: model too sensitive to the main parameter, wind speed; generally unsatisfactory comparison with earlier data taken at Point Beach (Ref. 21 of Appendix A).

2. Hayashi and Shuto: mainly historical nature of model; no jet entrainment simulated in this model.

3. Wada (Model No. 1): computer program unavailable.

4. Carter: mainly historical nature of model; jet model based on hydraulic data taken in too small a basin; improved data appeared recently in Ref. 25.

5. Koh and Fan (2D Model): no lateral entrainment. simulated in this two-dimensional (longitudinal, vertical) model; as with the Wada Model No. 2, it is basically a tool to develop greater physical insight.

6. Koh and Fan (axisymmetric model): discharge outfall assumed in this model is circular, not rectangular as exists at Point Beach and Palisades.

7. Barry and Hoffman: model authors prefer to make changes in model development before further application. 
8. McLay et al.: modification on Hoopes et al. model which includes a region of flow establishment and a constant spreading rate; not used because of generally unsatisfactory experience with computer code.

9. Stefan: no region of flow establishment, which severely restricts application of model; also, model comparison with Stefan's tank data not satisfactory (possibly due also to stratification of ambient water in tank).

10. Paul and Lick: new model with computer code incomplete at time of present study.

11. Engelund and Pedersen: model applicable only for surface discharges having large initial densimetric Froude numbers.

12. Waldrop and Farmer: model is of too recent an origin.

13. Wada (Model No. 2): no computer code available; no lateral entrainment simulated in this two-dimensional (longitudinal, vertical) model.

14. Sundaram et al.: mainly historical nature of model; jettrajectory formulas not applicable until reasonably distant from outfall.

15. Elliott and Harkness: model authors are presently improving their near-field analysis.

16. Giles et al.: model authors will be making further (though minor) modifications in the model; the hybrid computer required for model application is too large for Argonne facilities.

17. Loziuk et al.: model authors are presently modifying the hydraulics of their jet analysis; computer code presently unavailable.

18. Brady and Geyer: model is of too recent an origin for application in this report.

19. Till: model is of too recent an origin.

20. Pritchard (Model No: 2): model is of too recent an origin.

The four analytical models to be considered in this report and compared with the present field data are summarized next.

\section{A. Motz-Benedict Model}

Motz and Benedict have developed a two-dimensional model for the velocity and temperature distribution of a heated surface jet. ${ }^{5}$ Ambient receiving-water turbulence and buoyancy are assumed to be of minimal influence with regard to plume dynamics and heat transfer within the jet regime. The authors built their integral analysis upon the framework established primarily by Morton, ${ }^{26}$ Fan, ${ }^{27}$ Hoopes et al. ${ }^{1}$ and Carter. ${ }^{4}$ Conservation equations of mass, $x$ and $y$ momentum, and energy, along with two equations of jet bending, lead to a system of six ordinary differential equations which must be solved numerically to yield the jet trajectory, centerline temperature and velocity, and profile width. The Motz-Benedict model assumes that only two factors affect the flux of momentum at any 
TABLE II. Summary of Characteristics of Jet Models (ANL Neg. No. 190-990 Rev. 1).

\begin{tabular}{|c|c|c|c|c|c|c|c|c|c|c|c|c|c|c|c|c|c|c|c|c|c|c|c|}
\hline \multirow{2}{*}{\multicolumn{2}{|c|}{ 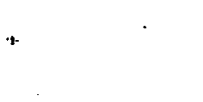 }} & \multicolumn{3}{|c|}{ 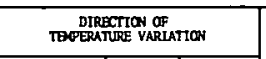 } & \multirow{2}{*}{ 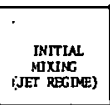 } & \multirow{2}{*}{ 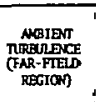 } & \multicolumn{4}{|c|}{ MATrFMTICAL APPROACH } & \multirow{2}{*}{ 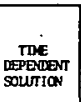 } & \multirow[b]{2}{*}{ sorver } & \multirow[b]{2}{*}{ coosis } & \multirow[b]{2}{*}{$\begin{array}{l}\text { Sorton } \\
\text { Stopt }\end{array}$} & \multirow{2}{*}{ 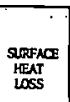 } & \multirow[b]{2}{*}{ 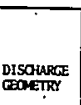 } & \multirow{2}{*}{ 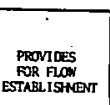 } & \multirow{2}{*}{ 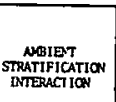 } & \multirow{2}{*}{ 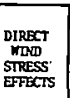 } & \multirow{2}{*}{ 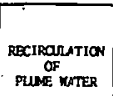 } & \multirow{2}{*}{\multicolumn{2}{|c|}{ 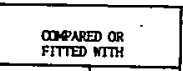 }} & \multirow[b]{2}{*}{ Praprise } \\
\hline & & Lavertorisus & utERAL & verrical & & & mexacal & INEGON & $\cos 2 \mathrm{~d}$ poss & SBR-EPIRICAL & & & & & & & & & & & & TAXX DATA & \\
\hline 1. & HCOFES 트 스. & ves & ves & no & yes & no & no & Yes & $\mathrm{No}^{2}$ & YES & so & no & res & $10^{\circ}$ & res & $\overline{\mathrm{YSES}}$ & $10^{\circ}$ & no & Yes & $\infty$ & YES & o & YeS $S^{3}$ \\
\hline 2. & HMTSSII NDO SATO & YeS & Yes & YES & res & no & no & YRS & Yes & YES & no & VES & so & no & res & YES & $\infty$ & no & No & $\overrightarrow{\mathrm{No}}$ & so & YES. & $\mathrm{Not}^{k}$ \\
\hline 3. & 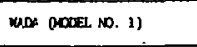 & YES & Yes & पres & Ves & no & ves & no & no & YES & VES & res & so & YeS & ViES & res & ס & so & so & res & unovon & Lasaiow & $\mathrm{yes}^{2}$ \\
\hline 4. & CAMER & Yes & nes & no & res & no & so & res & no & res & so & so & res & no & no & VES & res & no & סמ & so & No & YrES & YES \\
\hline s. & NOT: NAD BEXEDSCT & res & YES & no & YES & so & no & res & $N^{d}$ & Yes & א & n & YES & $\infty$ & TES & TES & Yes & no & so & מ & ves & YES & $\mathrm{nes}^{\mathrm{j}}$ \\
\hline 6. & KOH NND FN (2D KODEL) & yes & so & res & YES & so & no & res & no & res & No & YES & so & so & YES & VES & $\infty$ & w & Nof & No & $\infty$ & no & YeS \\
\hline 7. & 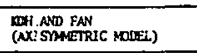 & res & VES & Yrs & Yres & so & no & Yes & no & YES & s & yes & so & no & YES & YES & so & no & no & so & n & so & res ${ }^{3}$ \\
\hline 8. & BNeTY AND HOFAMY & YES & Yes & YES & YES . & no & YES & so & No & res & so & res & res & res & YES & YES & סמ & $m$ & so & no & YrES & No & res' \\
\hline 9. & 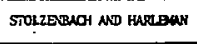 & YES & nES & res & res & no & so & res & no & res & no & YES & YES & No & TES & res & TES & so & so & so & No & res & $\mathrm{YES}^{j}$ \\
\hline 10. & 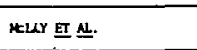 & riss & YES & nes & Yes & No & rese ${ }^{f}$ & resf & $\mathrm{nog}^{8}$ & VES & so & no & ress & so & Yes & Yes & Yes & s & $\mathbf{n}^{\circ}$ & no & ves & no & VES $S^{5}$ \\
\hline 1. & STEN & YES & YES & YES & VES & no & no & res & no & Yrs & no & yes & res & No & res & ress & no & so & res & so & no & YES & VES \\
\hline 12. & PeYra & Yes & YES & res & YES & no & s & YES & no & res & No & res & nes & $N$ & res. & res & YES & no & no & no & YES & Yes & $y=s^{3}$ \\
\hline 13. & PNAR AND LIX & YES & YES & VES & YES & no & VES & so & no & 谯 & ves & res & so & resi & res & $\overline{\text { YrES }}$ & $\overline{\text { YES }}$ & No & No & ness & so & no & Yes' \\
\hline 10. & EXELUNO NO FEESEST & res & nes & res: & res & so' & so & VES & ves & ves & res & $\infty$ & nes & so & s & s & ras & so & $\infty$ & so & no & YTS & $\mathrm{Not}^{k}$ \\
\hline 15. & mULAP AND BNOER & rES & $\mathrm{nes}$ & Yes & VEs & so & YES & so & $\infty$ & nis & nes & nes & $m$ & nes & nes & nes & VES & res & ves & ness & res & res & res \\
\hline
\end{tabular}

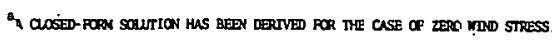

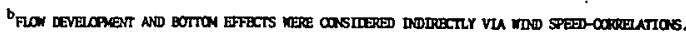

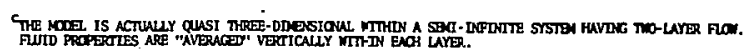

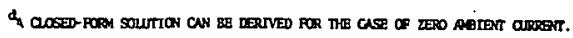

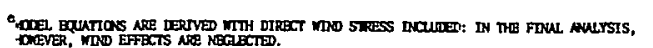

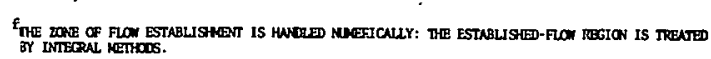

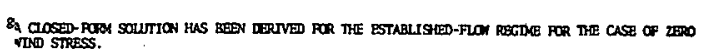

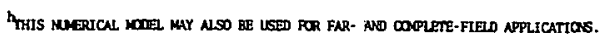

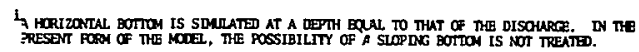

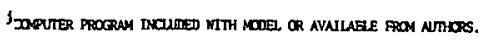

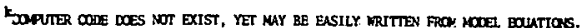

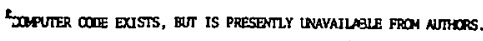

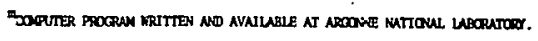


TABLE III. Summary of Characteristics of Complete-fieid Models (ANL Neg. No. 190-989 Rev. 1)

\begin{tabular}{|c|c|c|c|c|c|c|c|c|c|c|c|c|c|c|c|c|c|c|c|c|c|c|c|}
\hline & & \multicolumn{3}{|c|}{ 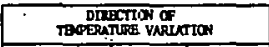 } & \multirow{2}{*}{ 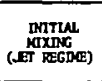 } & \multirow{2}{*}{ 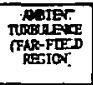 } & \multicolumn{4}{|c|}{ 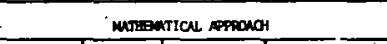 } & \multirow{2}{*}{ 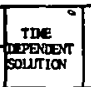 } & \multirow[b]{2}{*}{ sorater } & \multirow{2}{*}{ conss } & \multirow{2}{*}{ sorper } & \multirow{2}{*}{ 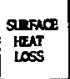 } & \multirow{2}{*}{ 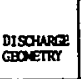 } & \multirow{2}{*}{ 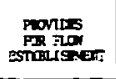 } & \multirow{2}{*}{ 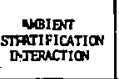 } & \multirow{2}{*}{ 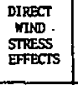 } & \multirow{2}{*}{ 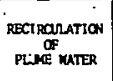 } & \multicolumn{2}{|c|}{$\begin{array}{l}\text { OOFARED NITH } \\
\text { FITIED XITH }\end{array}$} & \multirow{2}{*}{ 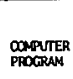 } \\
\hline & & Laxrinonive & LTENA & VERTEI & & & wesran & 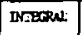 & COOSE PDOA & SPR-BPINTUL & & & & & & & & & & & FIED DATA & TANE DATA & \\
\hline 16. & $\max \cdot(\operatorname{cosect} x) \cdot 23$ & res & no & Vine & YES & res & YES & $\infty$ & $x$ & Ves & no & res & so & res & Iss & ves & $r$ & so & so & res & Uname & waromet & $\mathrm{res}^{\mathrm{a}}$ \\
\hline 11. & PRTtause noper No. 1) & res & vess & $r e$ & YES & res- & so & TES & Yres & res & $\infty$ & No & so & $\infty$ & TES & yes & $\mathrm{res}$ & \$ & - & Yes & yes & VES & $n=s^{b}$ \\
\hline 18. & sundarar EE 프. & ies & res. & Nc & VES & res & $\infty$ & no & VES & res & no & so & res' &.$\infty$ & $7 x^{x}$ & VES & $x$ & No & so & No & VES & no & ves \\
\hline 19. & ELLOTT AND HMPOESSS & yes & nes & Nc & VES & VES & No & TES & $x$ & yas & so & $\mathrm{No}^{\circ}$ & res & so & no & VES & s. & No & no & no & nes & so & $\mathrm{rns}^{\mathrm{n}}$ \\
\hline 20. & GIES EI N. ‥ & Yes & res & res & res & YES & res & TES & $x$ & res & $m$ & No & res & nes. & ves & res & not & N. & ves & Yes & Yes & so & $\mathrm{res}^{\mathrm{t}}$ \\
\hline a. & Lozrux 트스. & nes. & res & $x$ & VES & YES & Yres & .0 & $\mathbf{x}$ & nes & $\infty$ & no & res & no & VES & VES & No & No & so & so & res & ress & $\mathrm{rES}^{\mathrm{a}}$ \\
\hline 22. & BREOY ND GPVER & Yes & $\therefore$ TES & res & VES & nes & YES & so. & $\times$ & nes & as & res & Yes & YES. & VES & res & VES: & res & YES & ns & so & no & $\mathrm{res}^{\mathrm{f}}$ \\
\hline 23. & TIL & res & nes & ves & res & res & nes & no & $x$ & nes & $\Phi$ & mes. & $\mathrm{nes}$ & mes. & nes & ves & nEs: & no & so & res & res & no & $\mathrm{VES}^{\mathrm{f}}$ \\
\hline 24. & 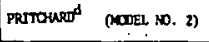 & res & TES & Yes & Yes & res & so & no & $n$ & ns & m & son & $\mathrm{ns}$ & is & $n$ & nes & Ex: & no & no & - & YES & YES & $\mathrm{rss}^{\mathrm{b}}$ \\
\hline
\end{tabular}

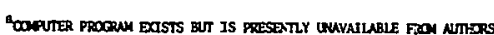

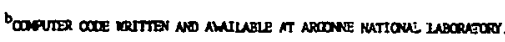

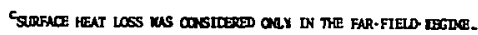

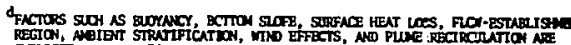

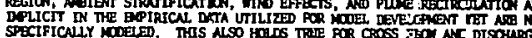

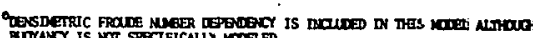

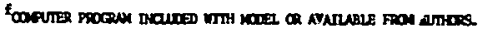


lateral slice of the jet: The first is the entrainment of lateral crossflow momentum, and the second is a net pressure force caused by eddying of the ambient fluid in the lee of the jet and by distortion of the jet boundaries. Explicit expressions must be assumed for both the entrainment function (to determine mass and momentum conservation) and the drag force per unit length (to determine momentum conservation). The assumed form of the entrainment velocity is

$$
v_{i}=E\left(U-U_{a} \cos \beta\right)
$$

where

$$
\begin{aligned}
\mathrm{v}_{\mathrm{i}} & =\text { inflow velocity (of entrainment), } \\
\mathrm{U} & =\text { local jet centerline velocity, } \\
\mathrm{U}_{\mathrm{a}} & =\text { ambient current velocity, } \\
\beta & =\text { local angle between jet centerline and ambient current, }
\end{aligned}
$$

and

$$
E=\text { entrainment coefficient. }
$$

The form of the assumed drag force is

$$
F_{D}=\rho \frac{C_{D} U_{a}^{2} z \sin \beta}{2},
$$

where

$$
\begin{aligned}
& F_{D}=\text { drag force operating normal to jet axis, } \\
& C_{D}=\text { experimentally determined drag coefficient, }
\end{aligned}
$$

and

$$
z=\text { jet depth. }
$$

This form for $F_{D}$ is based on the assumption that the interaction of the jet with the ambient current can be treated as if the jet were a solid body and the resulting pressure gradients can be represented by a drag force. Utilization of the model requires the specification of the drag and entrainment coefficients, $C_{D}$ and $E$, respectively.

In the region of established flow, the authors make similarity assumptions for velocity and temperature:

$$
u(s, \eta)=U(s) \exp \left(-\eta^{2} / b^{2}\right)
$$

and

$$
T^{\prime}(s, \eta)=T(s) \exp \left(-\eta^{2} / b^{2}\right),
$$


where

$$
\begin{aligned}
\mathrm{u} & =\text { local jet velocity, } \\
\mathrm{T}^{\prime} & =\text { local jet temperature, } \\
\mathrm{U} & =\text { jet cente rline velocity, } \\
\mathrm{T} & =\text { jet cente rline temperature, } \\
\mathrm{s} & =\text { distance along jet centerline, } \\
\eta & =\text { lateral distance from jet centerline, }
\end{aligned}
$$

and

$b=$ characteristic width of lateral profiles.

The assumed forms for $u, T^{\prime}, v_{i}$, and $F_{D}$ are used in the integral equations of conservation to yield the jet charracteristics in the established flow regime.

To account for the zone of flow establishment, Motz and Benedict have developed phenomenological relationships for. the length of the region of flow establishment $\left(s_{e}^{\prime}\right)$ and the initial angle of the jet at the end of the region of flow establishment $\left(\beta_{0}\right)$. These relationships are based on a series of laboratory hydraulic studies. No details of the temperature and velocity distribution in the region of flow establishment were determined, only its length and final angle with the shore. These studies yielded values for s', $\beta_{0}, C_{D}$, and $E$ in terms of the ratio $A$ of ambient to initial jet velocity and the actual initial angle of the jet with respect to shore, $\beta_{0}^{\prime}$. (The authors, however, recommend a value for $C_{D}$ of 0.5 be used for most cases.) Also needed is the width of the jet at the end of the region of flow establishment, $2 \mathrm{~b}_{0}$. The authors suggest a value calculated by equating the heat flux at the point of discharge with the heat flux at the end of flow establishment. The actual width at the point of discharge is denoted by $2 b_{0}^{\prime}$.

B. Stolzenbach-Harleman Model

The mathematical model of Stolzenbach and Harleman ${ }^{9}$ predicts the distributions of temperature and velocity within a completely determined jet structure for a near-field region, defined by the predominance of initial jet momentum over the effects of ambient lake turbulence.

Wherever possible, the model synthesizes previous knowledge of buoyant and norbuoyant jets. The heated discharge is assumed to be structured in its physical characteristics as well as its assumed velocity and temperature distribution, basically like a classical, turbulent, nonbuoyant jet. Just as for nonbuoyant jets, the authors assume an initial core region void of shear followed by the main turbulent region. The basic jet structure assumed by Stolzenbach and Harleman appears in Fig. 53. 

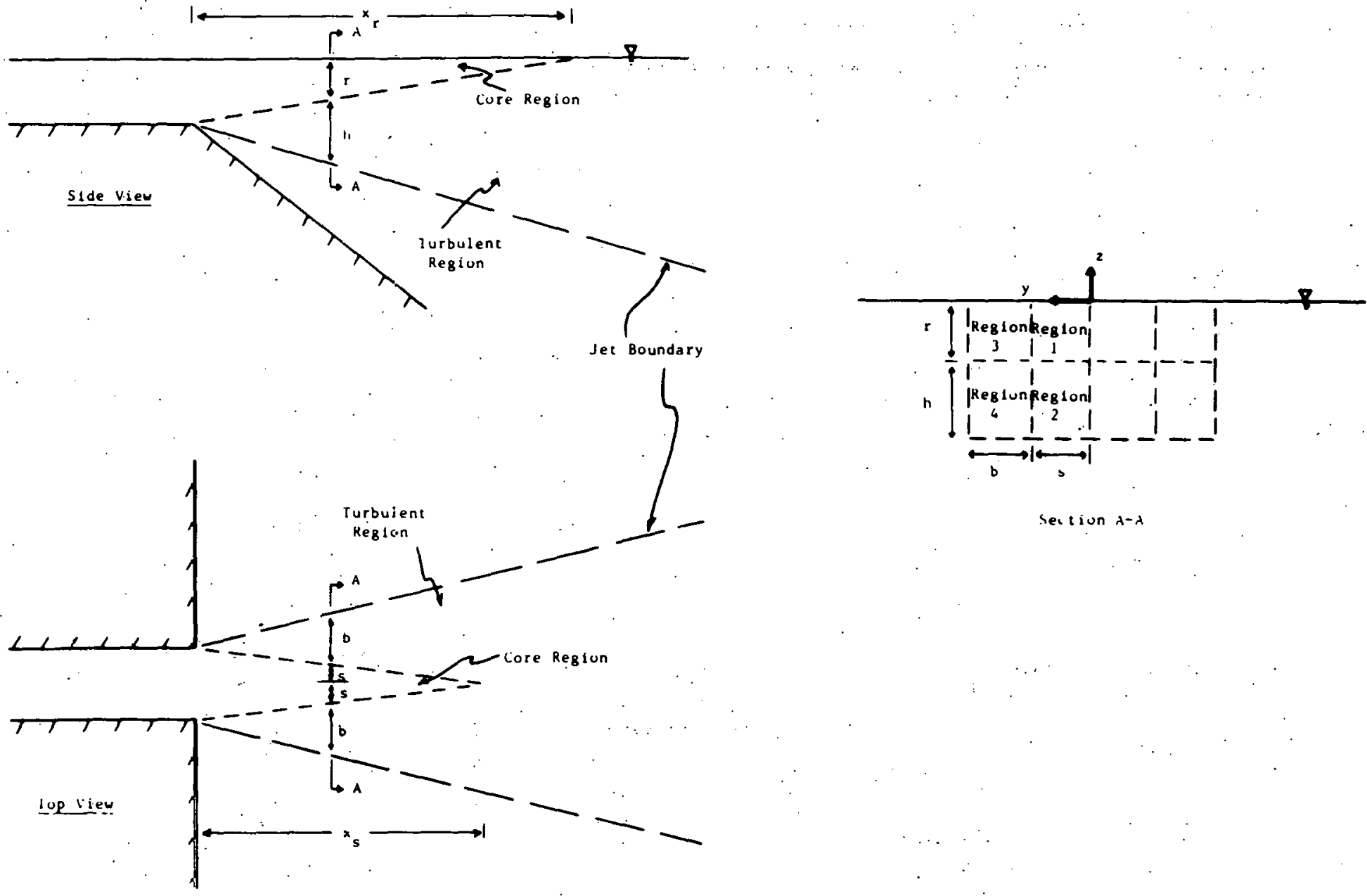

Fig. 53. Geometrical Characteristics of Jet Assumed in Stolzenbach-Harleman Model 
Using an integral method as in most jet analyses, velocities and temperatures at each longitudinal cross section are presumed to be related to centerline values by similarity profiles. Figure 54 illustrates the similarity profiles assumed. These are represented mathematically by

$$
u=u_{c}(x) F_{y}(y) F_{z}(z)-V \cos \theta
$$

and

$$
\Delta \mathrm{T}=\Delta \mathrm{T}_{\mathrm{c}}(\mathrm{x}) \mathrm{T}_{\mathrm{y}}(\mathrm{y}) \mathrm{T}_{\mathrm{z}}(\mathrm{z})
$$

where

u, $\Delta T$ - lucal value of velocity and temperature excess,

$\mathrm{x}, \mathrm{y}, \mathrm{z}=$ longitudinal, lateral, and vertical coordinates,

$\mathrm{V} \cos \theta=$ component of ambient velocity. normal to jet,

$$
\begin{array}{ll}
F_{y}=T_{y}=1.0, & 0<|y|<s, \\
F_{y}=f\left(\zeta_{y}\right), T_{y}=t\left(\zeta_{y}\right), & s<|y|<b+s, \zeta_{y}=\frac{|y|-s}{b}, \\
F_{y}=T_{y}=0, & b+s<|y|, \\
F_{z}=T_{z}=1.0, & -r<z<0, \\
F_{z}=f\left(\zeta_{z}\right), T_{z}=t\left(\zeta_{z}\right), & -r-h<z<-r, \zeta_{z}=\frac{-z-r}{h}, \\
F_{z}=T_{z}=0, & z<-h-r .
\end{array}
$$

The form of the similarity functions (from Abramovich ${ }^{28}$ ) are

$$
f=\left(1-5^{3 / 2}\right)^{2}
$$

and

$$
t=\sqrt{f}=1-\zeta^{3 / 2}
$$

The variables $b, s, h$, and $r$ are defined in Figo. 5.3 and 54.

Horizontal and vertical entrainment of ambient fluid is related to the jet centerline velocity by appropriate entrainment coefficients. The lateralentrainment coefficient is determined by nonbuoyant jet theory alone and is constant; the vertical-entrainment coefficient is related to the local temperature gradient by the local Richardson number, using the experimental results of Ellison and Turner, ${ }^{29}$ and is chosen to reduce to the nonbuoyant value when no density gradients exist. 

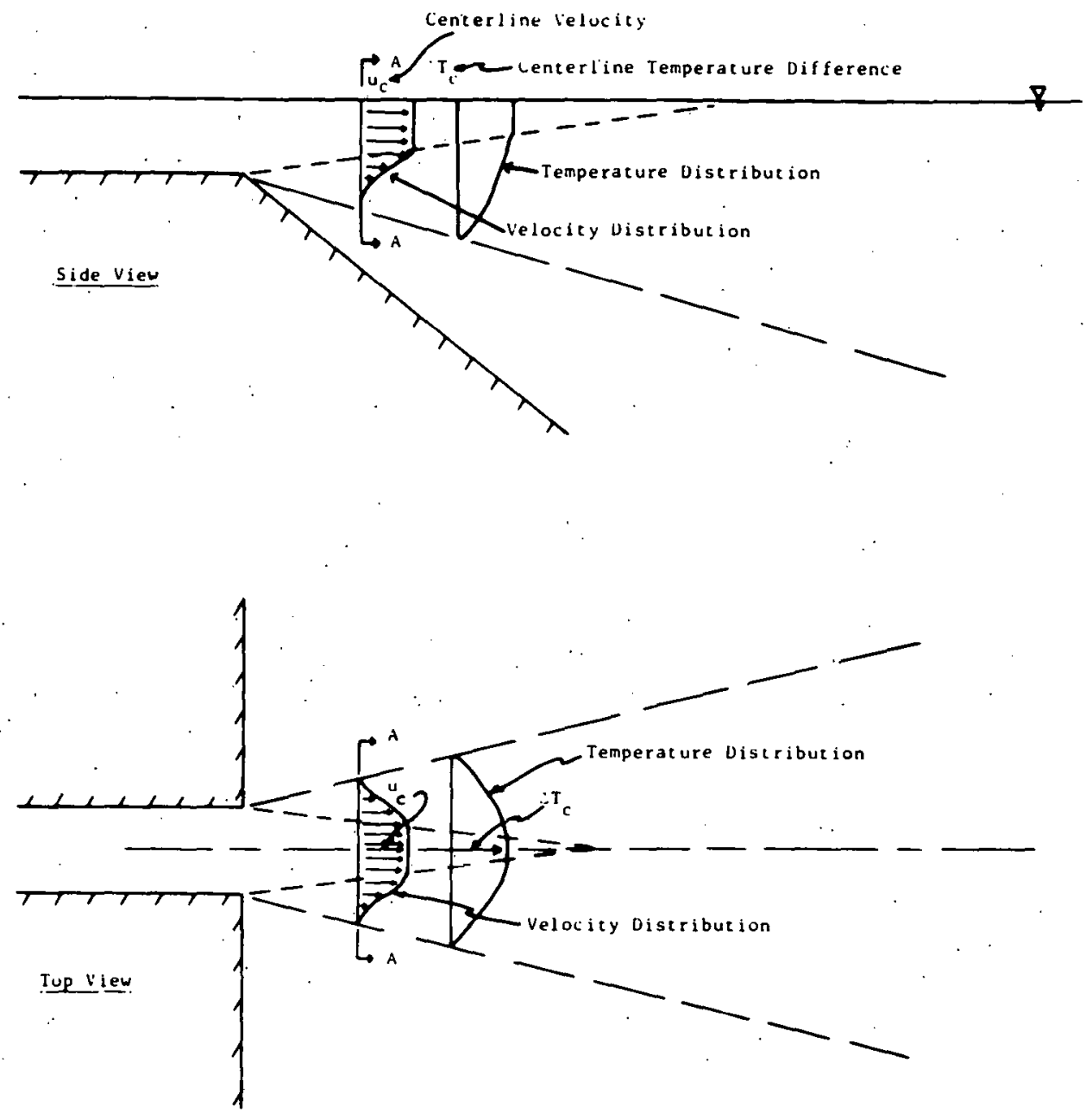

Fig. 54. Velocity and Temperature Characteristics of Jet Assumed in Stolzenbach-Harleman Model

Buoyant convection is incorporated through the pressure-gradipnt terms in the equations of motion and through the vertical-entrainment coefficient. Buoyancy effects generally reduce vertical entrainment and enhance lateral spreading.

To treat the interaction of these complex phenomena encompassing jet momentum, entrainment, buoyancy, and ambient crosscurrent, the authors developed their model from the steady, time-averaged differential equations of mass, momentum, and conservation of heat energy by dropping negligible terms, assuming a form for some of the unknown variables, and finally integrating the simplified equations over the four assumed regions of the jet longitudinal cross section.

Boundary conditions for the differential equations of mass, momentum, and energy (along with jet geometry) required as sumptions for the boundary values of heat and mass fluxes as well as for internal lateral and vertical velocity distributions. 
The set of conservation differential équations for a buoyant deflected jet are integrated over the jet cross section. The continuity and $x$-momentum equations are each integrated over all of the four regions defined in Fig. 53, yielding eight equations. The y-momentum equation is integrated over half the (symmetric) cross section to yield the ninth equation. The tenth is a jet-bending equation obtained from equating the rate of entrainment of lateral momentum to the rate of jet deflection. (No drag force on the jet is assumed.)

In this manner, the coupled flow and energy equations reduce to a system of simultaneous, first-order, nonlinear ordinary differential equations in the single variable $x$ (longitudinal distance along jet centerline from the outfall), which are then solved numerically. The solution to the model equations yields three-dimensional temperature and velocity predictions as well as the jet physical characteristics.

The complete solution may be written in nondimensional form as

$$
\left.\begin{array}{rl}
\frac{\mathrm{T}-\mathrm{T}_{\mathrm{a}}}{\mathrm{T}_{0}-\mathrm{T}_{\mathrm{a}}} & = \\
\frac{\mathrm{u}}{\mathrm{u}_{0}} & = \\
\text { teriotico } & =
\end{array}\right\} \text { functions }\left(\mathrm{F}_{0}, \mathrm{~A}, \frac{\mathrm{K}}{\rho \mathrm{c}_{\mathrm{p}} \mathrm{u}_{0}}, \frac{\dot{V}}{\mathrm{u}_{0}} \text {, and } \alpha_{0}\right),
$$

where

$$
\begin{aligned}
\mathrm{FF}_{0} & =\frac{\mathrm{u}_{0}}{\sqrt{\frac{\Delta \rho_{0}}{\rho_{\mathrm{a}}} \mathrm{gh} \mathrm{h}_{0}}}=\text { discharge densimetric Froude Number, } \\
\Lambda & =h_{0} / \mathrm{b}_{0}=\text { discharge-channel aspect ratio, } \\
\frac{\mathrm{I}}{\rho \mathrm{C}_{\mathrm{p}} \mathrm{u}_{0}} & =\text { surface-heat-loss parameter, } \\
\mathrm{V} & =\text { ambient crossflow velocity (possibly a function of offshore } \\
\mathrm{u}_{0} & =\text { channel outlet velocity, } \\
\alpha_{0} & =\text { angle of discharge with respect to shoreline, } \\
\mathrm{T}_{\mathrm{a}} & =\text { uniform lake water temperature, } \\
\mathrm{T}_{0} & =\text { initial discharge temperature, } \\
\Delta \rho_{0} & =\text { density difference between discharged and ambient water, }
\end{aligned}
$$




$$
\begin{aligned}
\rho_{a} & =\text { density of ambient lake water, } \\
g & =\text { acceleration due to gravity, } \\
2 b_{0} & =\text { width of discharge canal, } \\
h_{0} & =\text { depth of discharge canal, } \\
K & =\text { surface -heat-transfer coefficient, } \\
\rho & =\text { density of water }
\end{aligned}
$$

and

$$
c_{p}=\text { specific heat of water. }
$$

Thus the only site-dependent input parameters required are:

$$
\mathrm{IF}_{0}, \mathrm{~A}, \frac{\mathrm{K}}{\rho \mathrm{c}_{\mathrm{p}} \mathrm{u}_{0}}, \frac{\mathrm{V}}{\mathrm{u}_{0}} \text {, and } \dot{\alpha}_{0} \text {. }
$$

C. Prych Model

:The Prych model ${ }^{12}$ is based on a three-dimensional integral analys is of a turbulent, buoyant, horizontal, surface jet into a large, deep, uniform, turbulent flowing receiving water. Integral equations of conservation are written for mass, horizontal $x$ and y momentum, and energy, as well as equations for the jet trajectory. Figure 55 illustrates the coordinate systems and the jet region. The model uses Gaussian similarity assumptions for temperature and velocity:

$$
\begin{aligned}
& t(s, n, z)=T^{\prime}(s) \exp \left[-n^{2} / B^{2}(s)\right] \exp \left[-z^{2} / H^{2}(s)\right] \\
& u(s, n, z)=U(s) \exp \left[-n^{2} / B^{2}(s)\right] \exp \left[-z^{2} / H^{2}(s)\right]+V \cos \theta(s),
\end{aligned}
$$

where

$$
\begin{aligned}
t, u= & \text { local values of temperature excess and velocity, } \\
\mathrm{s}, \mathrm{n}= & \text { curvilinear coordinates along jet centerline parallel and } \\
& \text { perpendicular to the jet trajectory, respectively, } \\
\mathrm{z}= & \text { vertical distance from receiving-water surface, } \\
\mathrm{T}^{\prime}(\mathrm{s}), \mathrm{U}(\mathrm{s})= & \text { centerline values of excess temperature and excess } \\
& \text { velocity, } \\
\mathrm{B}(\mathrm{s}), \mathrm{H}(\mathrm{s})= & \text { local characteristic width and depth of jet, }
\end{aligned}
$$

and

$\mathrm{V} \cos \theta=$ component of ambient velocity parallel to jet trajectory. 

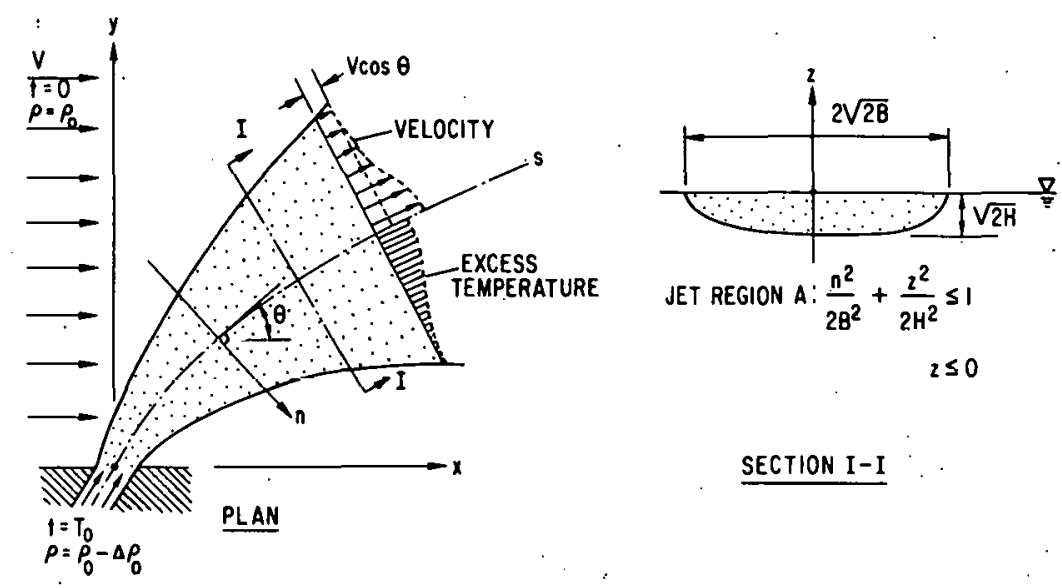

JET REGION A: $\frac{n^{2}}{2 B^{2}}+\frac{z^{2}}{2 H^{2}} \leq 1$

$2 \leq 0$

SECTION I-I

Fig. 66. Definition 3ketcli fur Cuordinate System and Jet Region Assumed in Prych Model. ANL Neg. No. 190-1045.

The rate of increase of the local jet flow due to incorporation of ambient wate $r$ is included in the model by simulating entrainment due to jet mixing and also entrainment caused by turbulence in the ambient fluid. Horizontal and vertical rates of entrainment are calculated separately for both processes. and are then summed. Prych computes jet entrainment in a manner similar to Stolzenbach and Harleman. Most notable is the requirement that the entrainment coefficient decreases with increasing fluid stability in the densitystratified jet. Prych calculates the contribution of ambient mixing to horizontal and vertical entrainment as $3.5 \epsilon / \sigma$, where $\epsilon$ is the ambient turbulent diffusion coefficient (horizontal or vertical) and $\sigma$ is the standard deviation of the lateral distribution of a tracer (excess temperature) in a two-dimensional jet. Prych assumes $\sigma=2 \sqrt{\mathrm{H}}$ for the vertical direction.

Four force terms appear in the $\mathrm{x}$ - and $\mathrm{y}$-momentum equations:

1. Ambient momentum flux in the direction of the ambient current.

2. Pressures due to the density differential between the fluid in the jet region and the ambient fluid. This force is assumed hydrostatic and is calculated by integrating the excess pressure force:

$$
\int_{-\infty}^{z} g \cdot \frac{\Delta \rho_{0}}{T_{0}}\left(s, n, z^{\prime}\right) d z^{\prime} \text { over each vertical cross-sectional face. }
$$

3. A form drag on the jet due to the pressure difference between offshore and lee sides of the jet, represented by

$$
F_{D}=\frac{1}{2} C_{D} \sqrt{2} H V|V| \sin ^{2} \theta
$$

where $C_{D}=$ form-drag coefficient.

This force is computed in the same way one computes form drag on a solid body. 
4. Interfacial shear stress between the jet fluid and the underlying ambient fluid. Prych approximates this force by modifying an expression for the shear stress at the base of a turbulent boundary layer on a smooth, flat, solid surface.

Once the basic conservation equations are integrated over the jet cross section using the above assumptions, a system of ordinary differential equations results that are solved for jet trajectory, jet width, jet depth, and jet centerline temperature and velocity. A separate region of flow establishment is included with the same forces simulated as described previously.

The basic input parameters to the model are:

$$
\begin{aligned}
Q_{0} & - \text { discharge flow rate from the outfall, } \\
T_{0} & =\text { excess temperature of jet (above ambient) at the outfall, } \\
\theta_{0} & =\text { angle of discharge velocity vector with positive } x \text { axis, } \\
2 b_{0}= & \text { width of outfall, } \\
h_{0}= & \text { depth of outfall, } \\
V & =\text { ambient-current velocity (assumed constant), } \\
\Delta \rho_{0}= & \text { difference in density between ambient water and water from } \\
& \text { outlet, } \\
E_{0}= & \text { surface-heat-transfer coefficient, } \\
C_{D}= & \text { form-drag coefficient, }
\end{aligned}
$$

and

$C_{F}=($ interfacial) shear-stress coefficient.

D. Pritchard Model

Pritchard's model ${ }^{17}$ is basically a synthesis of previous theoretical and physical-modeling results for buoyant and nonbuoyant jets, complemented by results the author has gleaned from field data obtained from existing power plants sited on bays, estuaries, and large lakes.

The model is simple and considers plume dispersion to be governed solely by momentum-jet entrainment, turbulent diffusion, and surface heat loss. An integral technique is used in which the plume velocity and excess temperature (above ambient) are assumed to have, at each longitudinal position, a "tophat" distribution laterally and vertically. Buoyancy-induced convective motions arc not cxplicitly coneidered. No ambient current is assumed to exist in the theoretical development; yet, the author expects that the predicted centerline-temperature decay and areas within isotherms 
will still be accurate in the presence of a current less than $10 \%$ of the initial discharge velocity. In the model, environmental changes are reflected solely in the surface-heat-transfer coefficient, $K$. Entrainment is accounted for by the specification of a fixed inverse spreading-rate parameter, $n$, normally taken to be about 6 . The lake bottom is assumed to have no effect other than upon the author's choice of a plume depth and, when necessary, upon the length and depth of an initial region of vertical entrainment.

The model handles both the jet and far-field regions; it predicts a two-dimensional temperature field and areas within isotherms down to a $0.56 \mathrm{C}^{\circ}\left(1.0 \mathrm{~F}^{\circ}\right)$ temperature excess. The parameters required for the application of this model are:

$$
\begin{aligned}
b_{0} & =\text { width of rectangular outfall, } \\
h_{0} & =\text { depth of rectangular outfall, } \\
\mathrm{Q}_{\mathrm{H}}{ }^{*} & =\text { excess heat-rejection rate of power plant based on } \theta_{0}, \\
\theta_{0} & =\text { initial excess temperature of the jet, }
\end{aligned}
$$

and

$$
K=\text { surface-heat-loss coefficient. }
$$

In spite of the simplifications made in the model development, the author claims the model to be conservative in many respects and simple to applỳ.

The author's theoretical development is carried out in four consecutive stages:

1. Horizontal spreading is considered, neglecting vertical diffusion and surface heat loss to the atmosphere. A two-dimensional temperature field is determined for the jet and far-field regions by the integral equations of conservation. Centerline temperature and velocity is found to drop off as $1 / \sqrt{s}$ in the jet regime, where $s$ is the distance along the centerline after a constant region of flow establishment of length $6 b_{0}$. For the far-field region, excess temperature is assumed to drop off as $1 / \mathrm{s}$.

2. Vertical entrainment is then considered independent of horizontal spreading and surface heat loss. The depth is assumed constant, except possibly for a small region in the vicinity of the outfall, where the depth grows slowly in a linear fashion. When vertical spreading is assumed to occur, a correction of the two-dimensional temperature field is made to account for the additional dilution. For Lake Michigan, Pritchard suggests that vertical

$* \mathrm{Q}_{\mathrm{H}}$ would be identically equal to the total condenser heat-rejection rate if the condenser intake temperature were identically equal to the ambient temperature. 
spreading be allowed for until the plume depth reaches $3.05 \mathrm{~m}$ ( $10 \mathrm{ft})$. For a greater initial depth, the jet is assumed to remain at that constant depth throughout the complete field.

3. A second correction of the temperature field may be needed, depending on the temperature of the water entrained' into the plume. Due to possible recirculation of condenser cooling water, the diluting water. mixed into the plume may have an excess temperature above ambient. Once this additional correction on the temperature field is made, the areas within isotherms can then be calculated for the condition of mixing alone.

4. Surface heat losses are then included in the analysis as a correction to the areas derived in stage 3 . This surface-heat-loss correction yields the final two-dimensional temperature field and the isotherm areas. 


\section{MODEL COMPARISONS TO DATA}

Table IV summarizes the basic discharge and environmental parameters required for application of the analytical models to the four Point Beach cases and the single Palisades case. Parameters such as entrainment and drag coefficients were chosen, based upon the recommendations of the model authors. In some cases (especially with the Motz-Benedict model), no clearcut choice exists for some of the required parameters. This problem is discussed in more detail in Sec. VIII.C. 2 below.

The data, JETFIT-smoothed results and model predictions are discussed and compared with respect to some of the major jet characteristice in the following paragraphs.

\section{A. Jet Trajectories}

Figures 56-60 show the jet trajectories resulting from JETFIT and the four model predictions. Before the analytical models are compared to the jet trajectory data, a few comments should be made rcgarding the JETFIT trajectories themselves. Figure 56 shows the jet centerline to bend gradually toward the north-shore direction (negative $\mathbf{x}$ direction). In a similar fashion, Fig. 57 shows the jet to bend gradually toward the south-shore direction (positive $\mathbf{x}$ direction). In both cases, the ambient current has been assigned a nominal value of zero, based on field measurements. Possible causes of the seemingly anomalous behavior may be combinations of the following:

1. A small but undetectable ambient current, directed north on May 18 and south on May 23, may have been present. The threshold of the instrument used for measuring ambient current is ahout. $3.0 \mathrm{~cm} / \mathrm{sec}$; consequently, any current below or near this value is virtually undetectable. Further, the ambient-current conditions were measured at a limited number of specific locations. A small current could possibly have been deter.ted at some other location, since near-shore current measurements are known to be spatlally and temporally unsteady.

2. Local gyres might exist in the region of the outfall. The intrusion of the $33-\mathrm{m}$. discharge structure into a small ambient-current field will cause local eddies and gyres to form in the vicinity of the discharge. Data taken near the outfall may be influenced by them. Also, a return current on the lee side of the jet (when an ambient current exists) or on both sides of the jet (when no ambient current is present) is required to provide water to the jet as it disperses due to jet entrainment and ambient mixing. When an ambient current exists, the region between the outer boundary of the jet on the lee side and the shoreline will be likely to contain eddies of continually recirculating water.

3. Lake-bottom irregularities in the vicinity of the discharge might influence the jet trajectory. The contours in Fig. 6 represent average values 
TABLE IV. Jata and Paraneters Used for Model Calculations for Foint. Beach and Palisades (ANL Neg. No. 190-956 Rev. 1)

Point Beach Unit No. 1

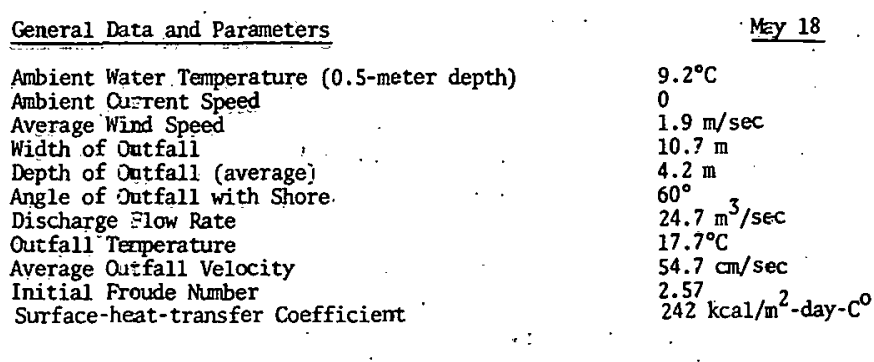

$14.3^{\circ} \mathrm{C}$
0
$1.0 \mathrm{~m} / \mathrm{sec}$
$10.7 \mathrm{~m}$
$4.2 \mathrm{~m}$
$60^{\circ}$
$25.1 \mathrm{~m}^{3} / \mathrm{sec}$
$21.6^{\circ} \mathrm{C}$
$55.5 \mathrm{~cm} / \mathrm{sec}$
$2.37 \mathrm{~cm}$
$197 \mathrm{kcal} / \mathrm{m}^{2}$-day- $\mathrm{C}^{\circ}$

$\quad$ July 13
$13.0^{\circ} \mathrm{C}$
$5 . ? \mathrm{~cm} / \mathrm{sec}$
$1.5 \mathrm{~m} / \mathrm{sec}$
$10.7 \mathrm{~m}$
$4.2 \mathrm{~m}$
$60^{\circ}$
$24.7 \mathrm{~m}^{3} / \mathrm{sec}$
$20.3^{\circ} \mathrm{C}$
$54.7 \mathrm{~cm} / \mathrm{sec}$
$2.42 \mathrm{mci} / \mathrm{m}^{2}$-day- $\mathrm{C}^{\circ}$
$166 \mathrm{kca} / \mathrm{m}^{-}$

September 9
$16.3^{\circ} \mathrm{C}$
$2.2 \mathrm{~cm} / \mathrm{sec}$
$3.7 \mathrm{~m} / \mathrm{sec}$
$10.7 \mathrm{~m}$
$4.2 \mathrm{~m}$
$60^{\circ}$
$24.7 \mathrm{~m}^{3} / \mathrm{sec}$
$24.5^{\circ} \mathrm{C}$
$54.7 \mathrm{~cm} / \mathrm{sec}$
$2.06 \mathrm{scal} / \mathrm{m}^{2}$-day- $\mathrm{C}^{\circ}$
$459 \mathrm{kca} / \mathrm{m}^{-}$

Palisades

- October 10 $13.0^{\circ} \mathrm{C}$ $2.0 \mathrm{~m} / \mathrm{sec}$ $2.0 \mathrm{~m} / \mathrm{sec}$
$28.3 \mathrm{~m}$ $2.1 \mathrm{~m}$ $25.6 \mathrm{~m}^{3} / \mathrm{sec}$ $42.3 \mathrm{~cm} / \mathrm{sec}$ $281 \mathrm{kcal} / \mathrm{m}^{2}$-day- $\mathrm{C}^{\circ}$

\section{Additional Parameters for Pritchard Mode1}

Inverse Spreading Rate

Temperature Excess of Recirculated Water

$\begin{array}{ll}6.0 & 6.0 \\ 4.2 \mathrm{~m} & 4.2 \mathrm{~m} \\ 0 & 0\end{array}$

Additional Parameters for Motz-Benedict Model

Length of Flow-establishment Region

Angle at End of Flow-establishment Region

Entrairment Coefficient

$.2 \mathrm{~m}$

6.0
$4.2 \mathrm{~m}$
0

$4.0 \mathrm{~m}$

6.0
$3.0 \mathrm{~m}$
0

Drag Coefficient

$\begin{array}{ll}55.5 \mathrm{~m} & 55.5 \mathrm{~m} \\ 60^{\circ} & 60^{\circ} \\ 8.5 \mathrm{~m} & 8.5 \mathrm{~m} \\ 0.05 & 0.05 \\ 0.5 & 0.5\end{array}$

$30.4 \mathrm{~m}$
$49.9^{\circ}$
$8.5 \mathrm{~m}$
0.307
0.5

0

Additional Parameters for Stolzeabach-Harleman Nodel

Aspect Ratio

0.79

0.79

0.79

0.151

Additional Parameters for Prych Model

Entrainment Coefficient

prag Coefficient

$\begin{array}{ll}0.1 & 0.1 \\ 0.2 & 0.2 \\ 0.5 & 0.5\end{array}$

0.1

0.1 


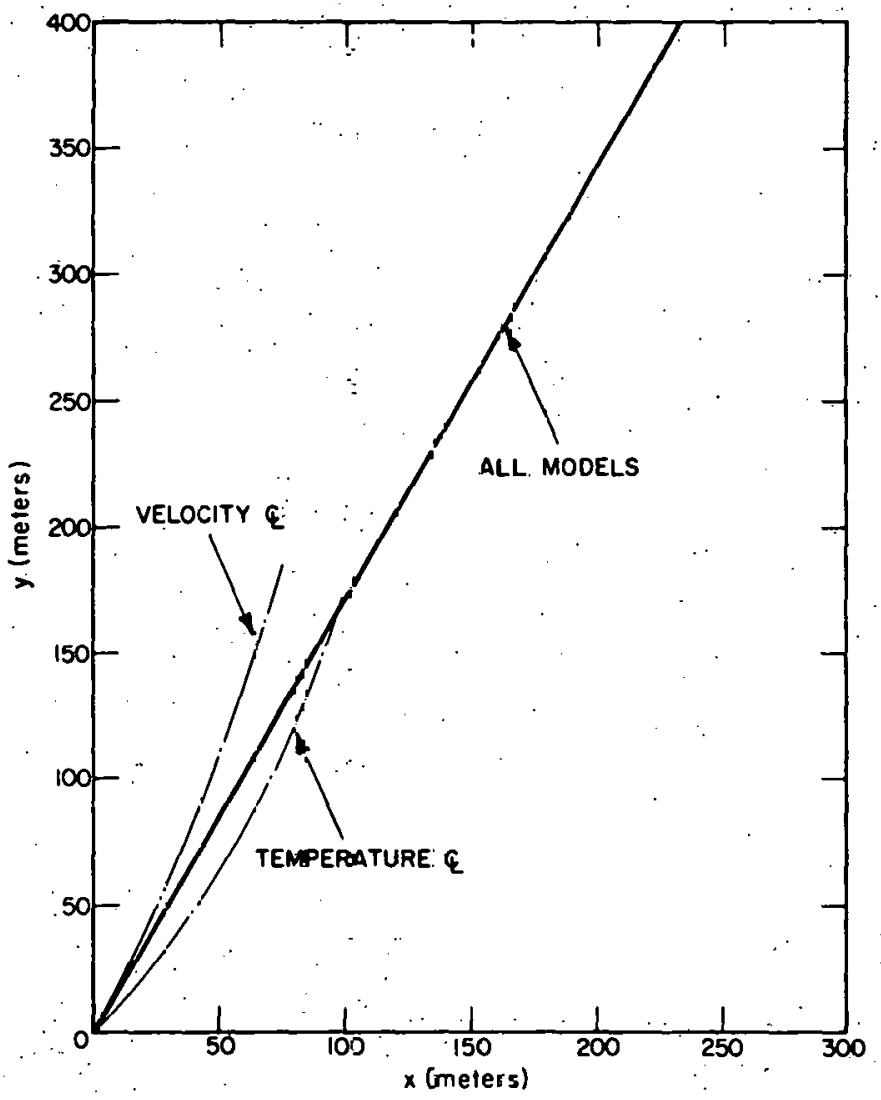

Fig. 56. Centerline Trajectories Resulting from the Fitting Procedure and Model Calculations for Point Eeach: May 18, 1๕72. ANL Neg. No. 190-935.

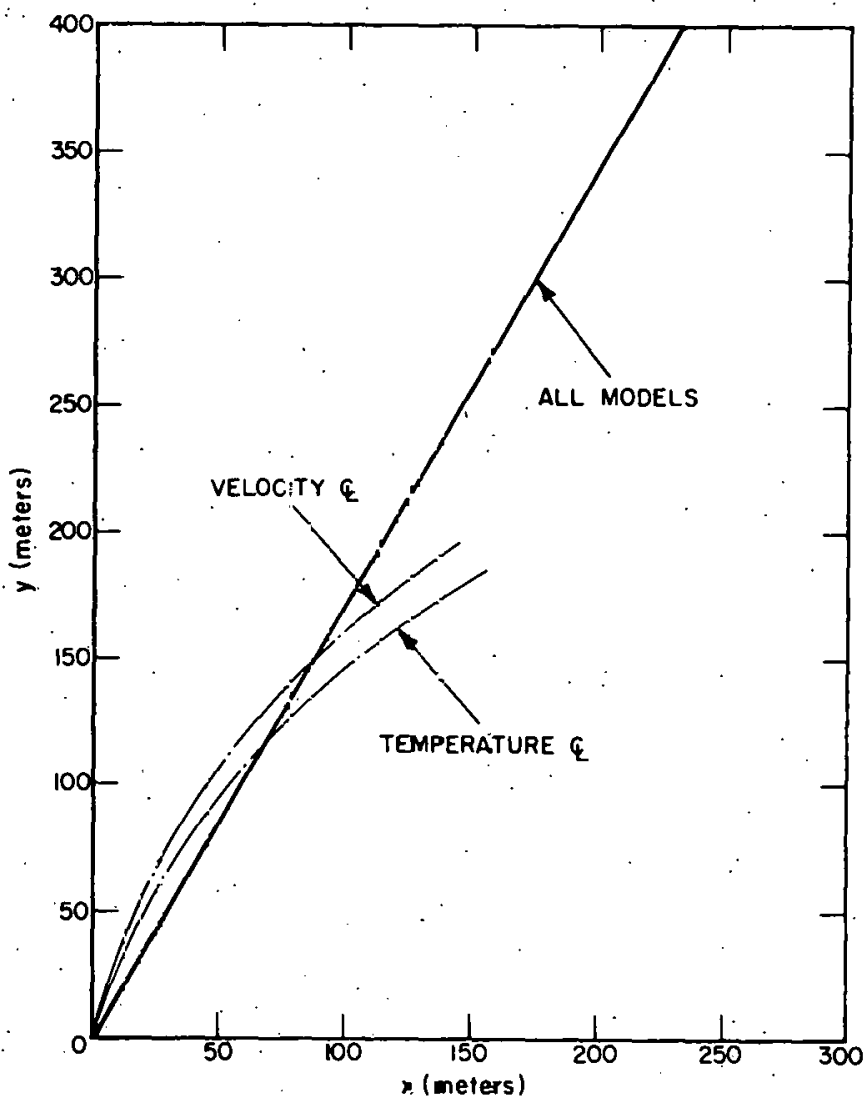

Fig. 57. Centerline Traject ries Resulting from the Fitting Procedure and Modəl Caiculations for Point Beach: May 23, 19?2. ANL NeE. No. 190-936. 


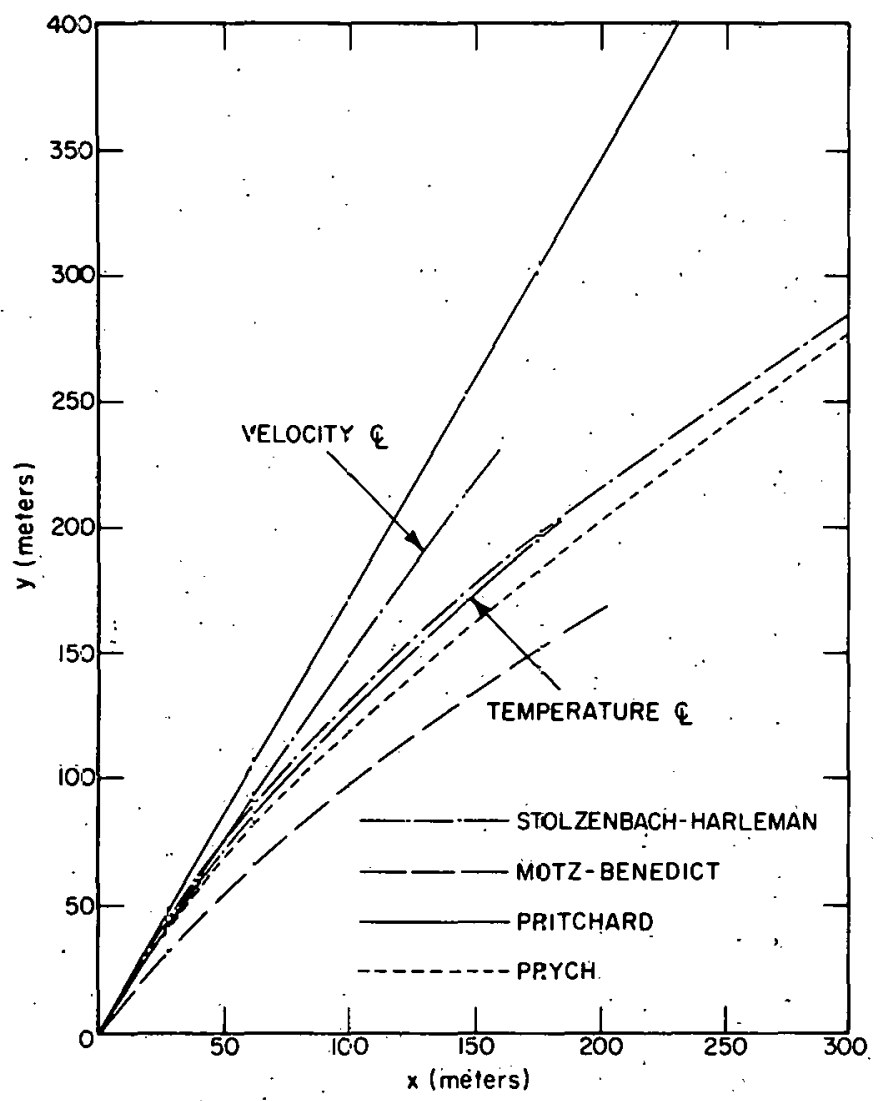

Fig. 58. Centerline Trajectories Restiting from the Fitting Procedure and Model Calculations for Point Beach July 13, 1972. ANL Neg. No. 190-940.

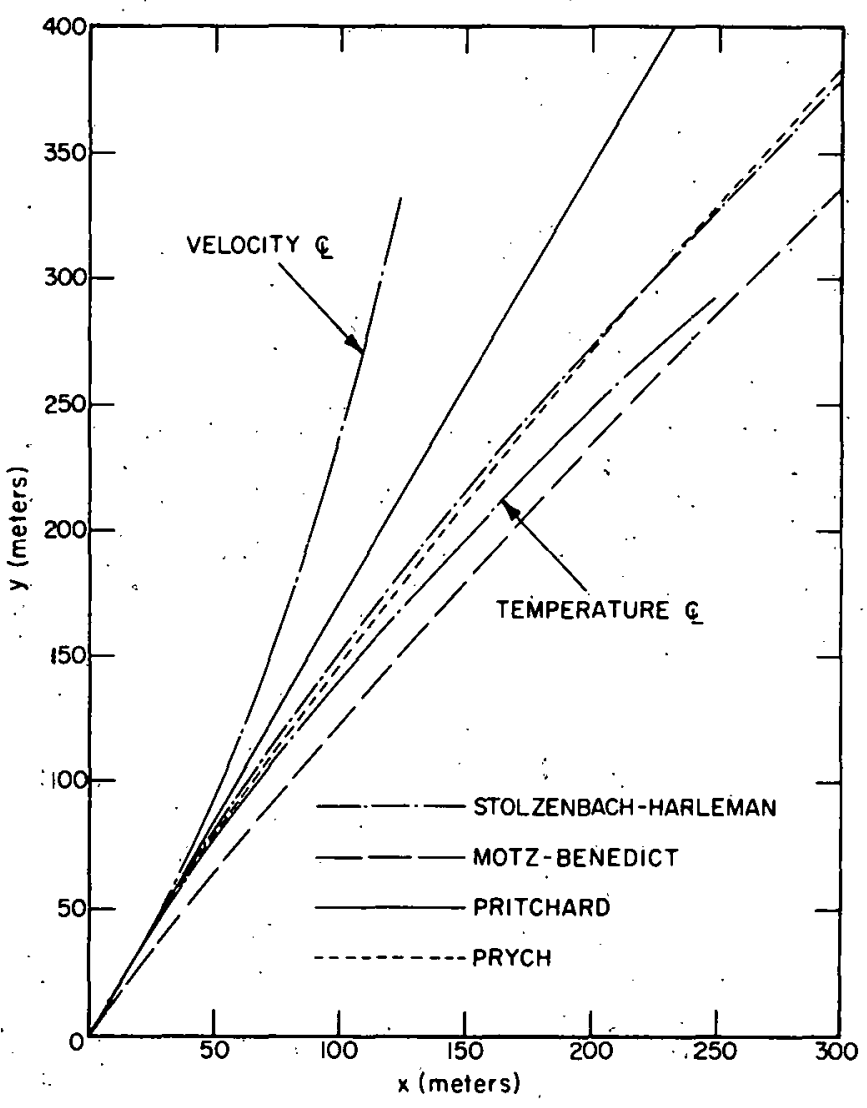

Fig. 59. Centerline Trajectories Resulting from the Fitting Pròcedure and Model Calculations for Point Beach: September 9, 1972. ANL Neg. No. 190-948. 


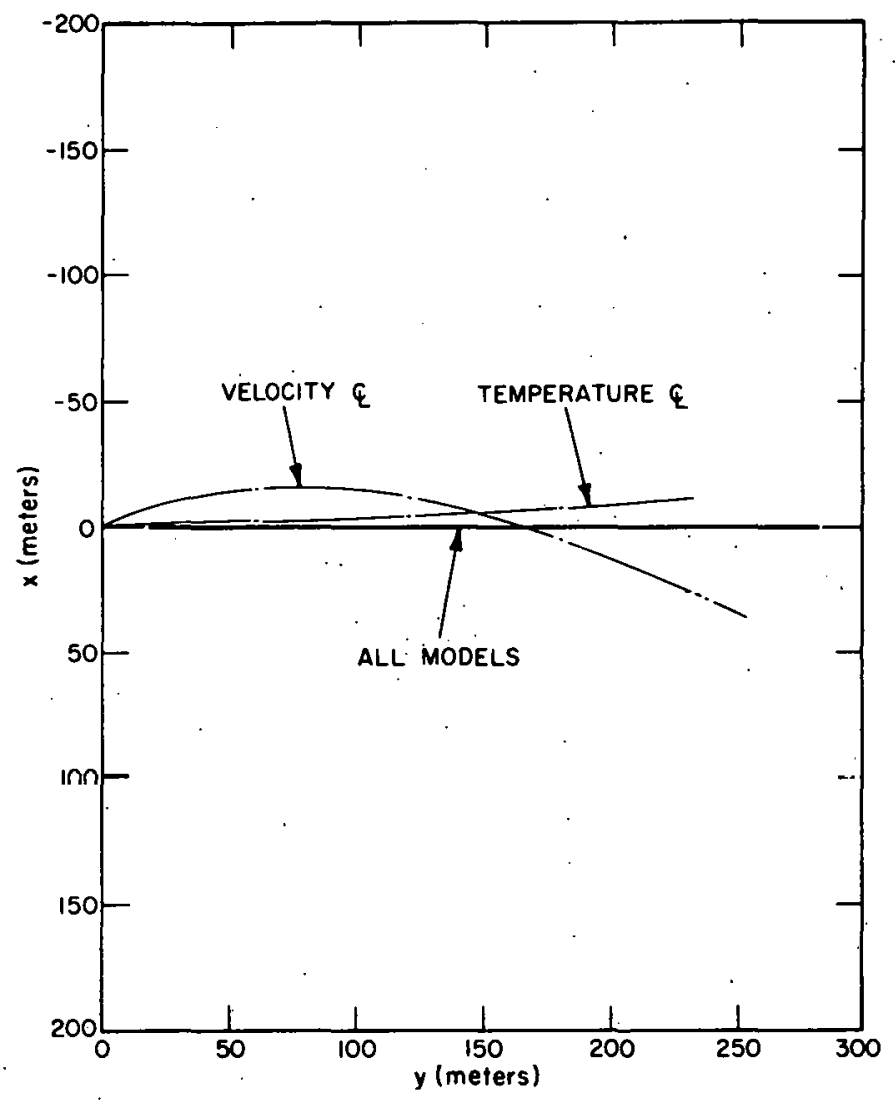

Fig. 60. Centerline Trajectories Resulting from the Fitting Procedure and Model Calculations for Palisades: October 10, 1972. ANL Neg. No. 190-957. of bottom soundings taken at several different dates in 1972. Continual operation of the Point Beach Unit 1 discharge has apparently dug out a small channel directly off the discharge. A small ridge appears north of the discharge canal, as well as a small bar just south of the outfall. Changes in the local bottom contours are possible, due to occasional storms in the area and daily variation in both ambient current and discharge conditions.

4. The fitting of symmetric profiles of temperature and velocity, as used in JETFIT, to distributions that are probably unsymmetric when an ambient current exists, may influence the apparent location of the centerline. Figure 61 illustrates a somewhat more realistic profile of centerline excess temperature $\theta / \theta_{0}$ and jet-centerline-velocity ratio $\mathrm{u} / \mathrm{u}_{0}$ (in the lateral direction) for a bent jet near the outfall. The profiles are based on hydraulicmodel measurements by $C$ arter ${ }^{25}$ for an outfall angle $\beta_{0}$ of $60^{\circ}$ and a ratio of outfall to' ambient current, $R$, of 2.0. The JETFIT data discussed here are for $R=10$ to $\infty$ (i.e., weak currents), which should reduce but not eliminate the asymmetry. of the velocity and temperature profiles shown in the figure. Any asymmetry due to an ambient current should bias the centerline fit to the

- Fig. 61

Idealized Surface Profile of Excess Temperature $\theta / \theta$, and Velocity $u / u_{0}$ across (y direction) Bent Jet near Orifice (based on measurements). $R=2$ (nominal) and $B_{0}=80^{\circ}$ (Rer. 25).

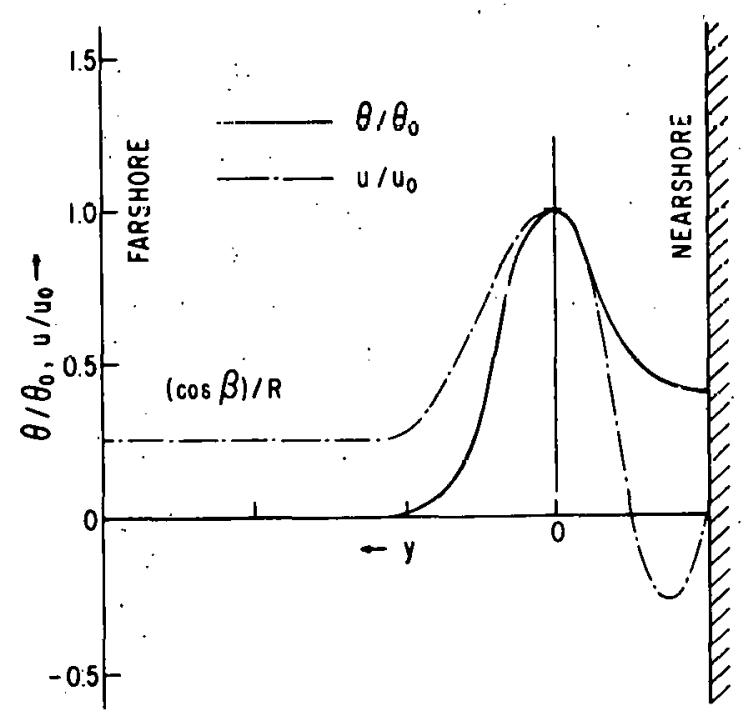


lee direction for the temperature centerline and to the offshore direction for the velocity centerline. This might explain the relative orientation of velocity and temperature centerlines for May 23, July 13, and September 9 Point Beach data (Figs. 57-59). The velocity centerline is also upstream of the temperature centerline for the fitting results at the other depths on these dates; discrepancies when occurring were very small and could, however, just as easily have been caused by insufficient data. Far-field temperature measurements'taken several hours later at 1645-1751 hours on May 18 (not presented here) indicate a southerly directed plume under the influence of a small ambient current. The results of May 18 seem to be largely biased by data measurements at Station No. 5, where the plume appears to be directed northward. There is the possibility of a small wind-induced current directed north existing near the third transect offshore in opposition to a southerly moving ambient current. Also, note that Station. No. 7 with zero velocity was eliminated from the JETFIT calculation, since it was not thought to be a point in the thermal plume. This would bias the velocity centerline in a southerly direction on May 18. More data are necessary to more accurately define the centerlines in this case.

5. Uncertainties in the data (up to $\pm 0.5 \mathrm{C}^{\circ}$ for temperature, $\pm 20 \%$ for velocity magnitude, $\pm 12^{\circ}$ of velocity direction) and the limited amount of data available clearly influence the fitting results. The temperatures recorded were averaged over 1 - to 2 -min time intervals; thermal fronts have been observed (Ref. 30) that can change the plume temperature at a fixed point at Point Beach up to $3 \mathrm{C}^{\circ}$ in $1-2$ sec. The appearance and frequency of such fronts (estimated to be from $30 \mathrm{sec}$ to several minutes) will be important in any given averaged measurement. Determination of ambient temperatures. and currents is also important to the analysis, yet are difficult to determine in the field.

Several of the general comments given in paragraphs $2-5$ above also apply to the jet data of July 13 and September 9. On September 9, Unit 2 at Point Beach was operating at $12 \%$ power.with only one of the two available. pumps. This corresponds to a flow rate of about $14 \mathrm{~m}^{3} / \mathrm{sec}$ with a tempera-. ture excess above ambicnt of about $2 \mathrm{C}^{\circ}$. Clearly, the velocity distribution should be affected by this second discharge, with the temperature field perturbed only slightly. The nonlinear interaction of flow and temperature fields complicates any further evaluation of resultant effects.

Analytical model predictions for May 18 and May 23 at Point Beach were based on a zero-ambient-current value. Consequently, a straightline trajectory was predicted by all models. The JETFIT results for these dates yielded only slight differences from a straight-line trajectory, probably due to the reasons given previously. Models will be compared to the temperature centerline of the jet, since it is generally of greater interest than the velocity centerline. 
The Stolzenbach-Harleman model and the Prych model appear most accurate for the dates with a measured ambient current. Prych predicts greater bending than Stolzenbach and Harleman for both dates. The Pritchard model suggests only a small change in trajectory from a straight line for situations in which a small ambient current exists. Consequently, a straightline trajectory is shown for Pritchard in Figs. 58 and 59. The Motz-Benedict model overpredicts jet bending for both dates. As stated above, the model is very sensitive to the value of the entrainment coefficient chosen.

Interpreting the Palisades results is difficult for several reasons.

First, the lake bottom in the vicinity of the discharge is very shallow, as may be seen in Fig. 7. This may inhibit any significant vertical entrainment. For the first $100 \mathrm{~m}$, the lake bottom is at approximately the depth of the outfall. At the north end (positive y-direction) of the diverging Palisades outfall, lake water occasionally enters the discharge channel. The discharge cross section is highly irregular, as sketched in Fig. 62. The discharge width, $b_{0}$, was chosen as $28.3 \mathrm{~m}$, since it is the channel width at the lake opening. The discharge depth $h_{0}$ was chosen to be

$$
h_{0}=\frac{\text { discharge area }}{b_{0}}
$$

the calculated $h_{0}$ was then averaged for the three dates shown in Fig. 62.

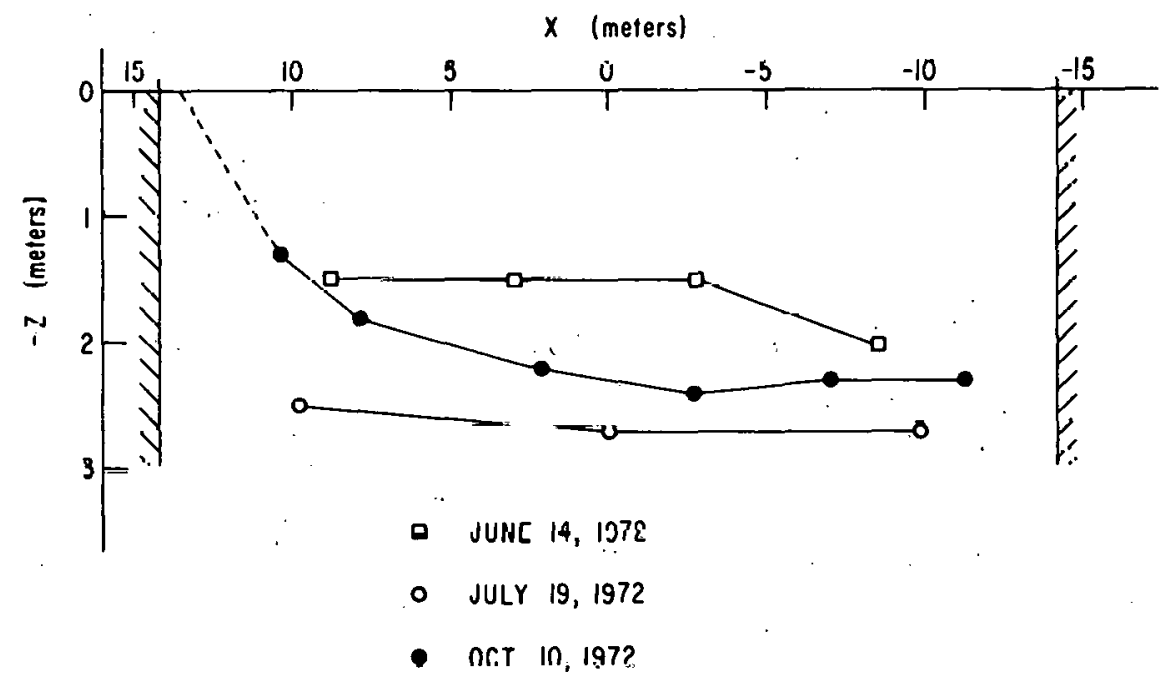

Fig. 62. Bottom Depth at Palisades Outfall for Three Dates of Jet Studies

Considering the significant bottom interaction that undoubtedly occurs in lhis case, all model predictions will be considered qualitatively only. Zero ambient current on October 10 clearly implies no bending for model predictions; JETFIT results indicate little deviation from this position (see Fig. 60). 
B. Centerline Temperature Decay. and. Temperature Half-widths

The centerline excess temperatures resulting from JETFIT and the four model predictions are shown in Figs. 63-67. The assumed parametric temperature profile to which the jet data was fitted with JETFIT is again

$$
\theta(\eta, s)=\theta_{c}(s)\left(\frac{1}{2}\right)^{\left[\eta / w_{T}(s)\right]^{2}},
$$

where

$$
\begin{aligned}
& \theta_{c}(s)=\theta_{0} A \quad \text { for } s \leq \frac{\alpha b_{0}}{A^{2}} \\
& =\theta_{0} \sqrt{\frac{\alpha \mathrm{b}_{0}}{\mathrm{~s}}} \text { for } s>\frac{\alpha \mathrm{b}_{0}}{\mathrm{~A}^{2}} \\
& W_{T}(s)=C b_{0}+Y s,
\end{aligned}
$$

and $A, \alpha, C$, and $\gamma$ are fitted parameters. It is clear from Figs. 63-67 that the temperature excess near the outfall from the data-smoothing procedure can be different from $\theta_{0}$. This corresponds to $A \neq 1$.

\begin{tabular}{|c|c|c|c|c|}
\hline & May 18 & May 23 & July 13 & September. 9 \\
\hline$A$ & 0.84 & 0.84 & 0.92 & 0.79 \\
\hline$\alpha$ & 3.8 & 5.6 & 6.2 & 4.5 \\
\hline$s_{0}$ & 5.38 & 7.95 & 7.32 & 7.22 \\
\hline
\end{tabular}

The values of $A, \alpha$, and length of region of flow establishment $s_{0}=\alpha / A^{2}$ (in units of the outfall width) for the four. Point Beach dates considered are:

The values of $s_{0}$ do compare with values in the range from 5 to 7 usually quoted in the literature. However, the above Point Beach numbers certainly reflect insufficiencies in the data and inadequacies in the fitting procedure; consequently, much significance should not be attached to them.

Since $\theta \neq \theta_{0}$ (i.e., $A \neq 1$ ) at the uutfall, one should not place much significance on the results of the fitting procedure for the first $50-100 \mathrm{~m}$. It may have been more profitable to have $A$ fixed at 1.0 and introduce a new parameter $\epsilon$ as the power of $\alpha \mathrm{b}_{0} / \mathrm{s}$ in the formula for $\theta_{c}(\mathrm{~s})$. (The present formulation restricts $\epsilon$ to a value of $1 / 2$.) The fit would then involve $\alpha, \epsilon$, $C$, and $Y$. It is not expected, however, that the present JETFIT results would be altered significantly if such changes were implemented in the fitting function. One further comment: The results of JETFIT for the jet characleristics most generally agreed with what one would expect from visual examination of the data along each transect of the jet. This gives additional confidence to the JETFIT reoults. 


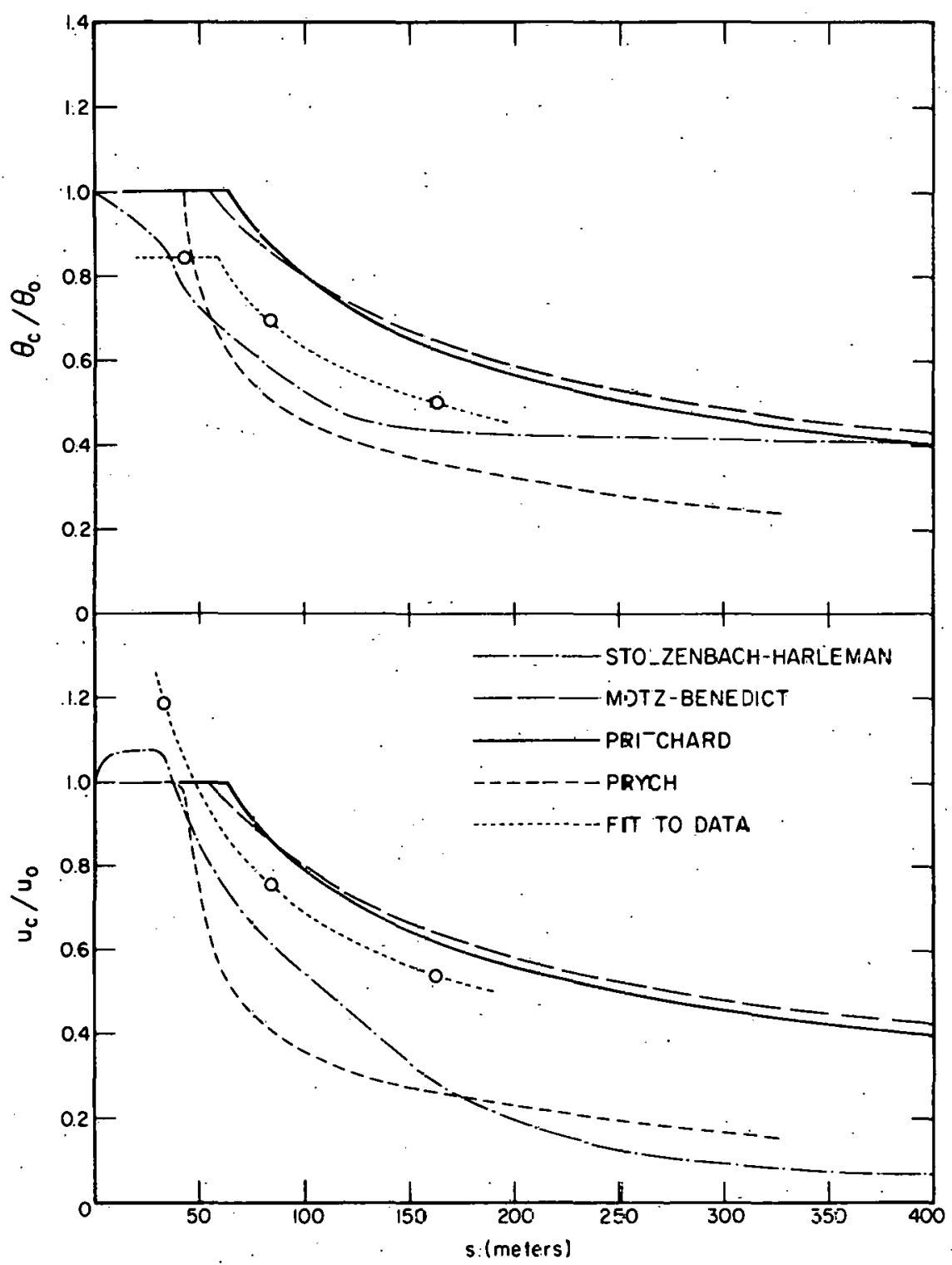

Fig. 63. Centerline Temperamure Excess and Velocity Decays Fesulting fram the Fitting Procedure and Model Calculations for Point Beach: May $1 \varepsilon, 1972$. ANL Neg. No. 190-952.

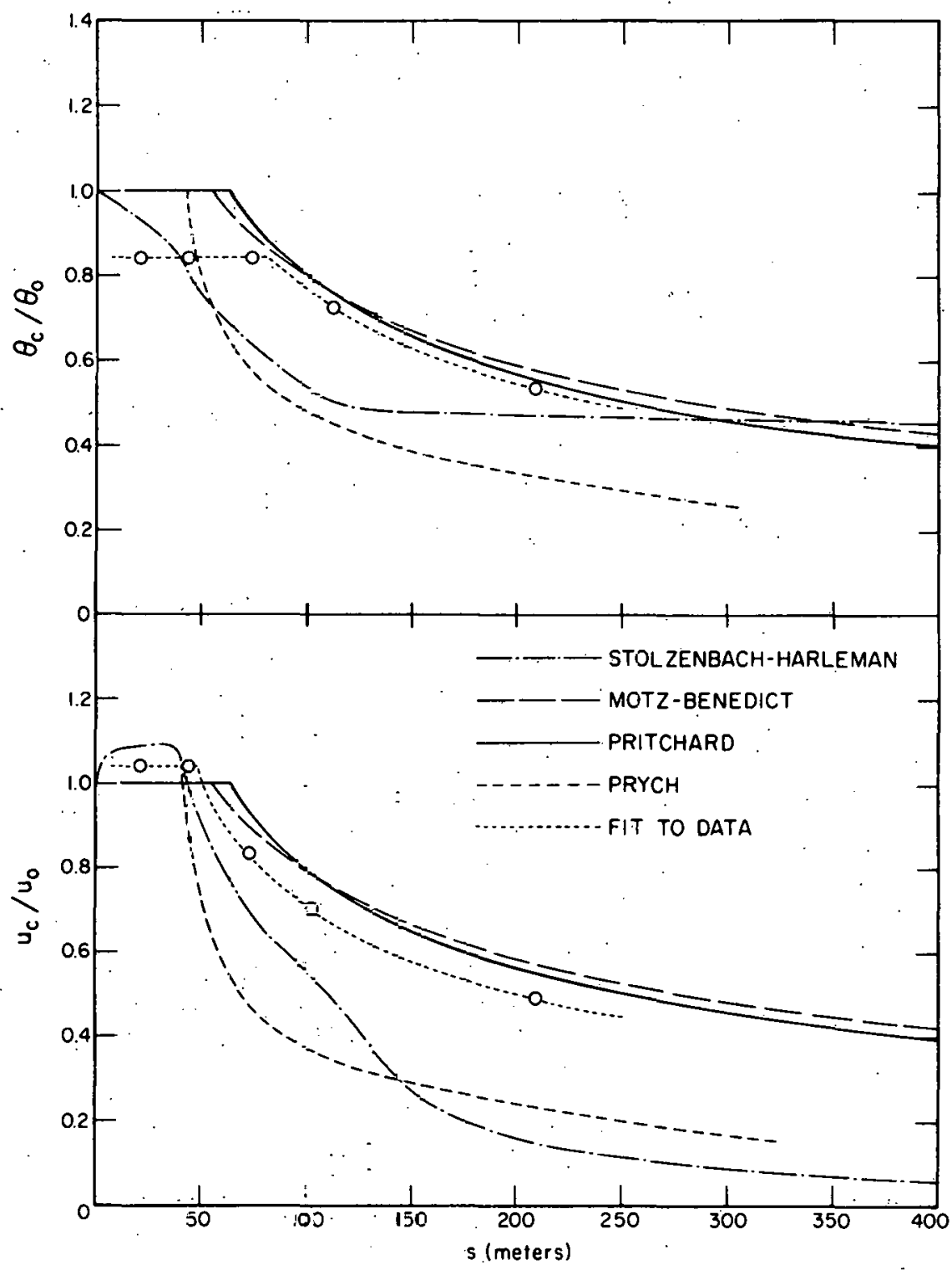

Fig. 64. Centerline Temferature Excess and Velocity Decays Resulting from the Fitting Procedure and Model Calculations for Point Beact:- May 2s, 1972. ANL Neg. No. 190-949. 


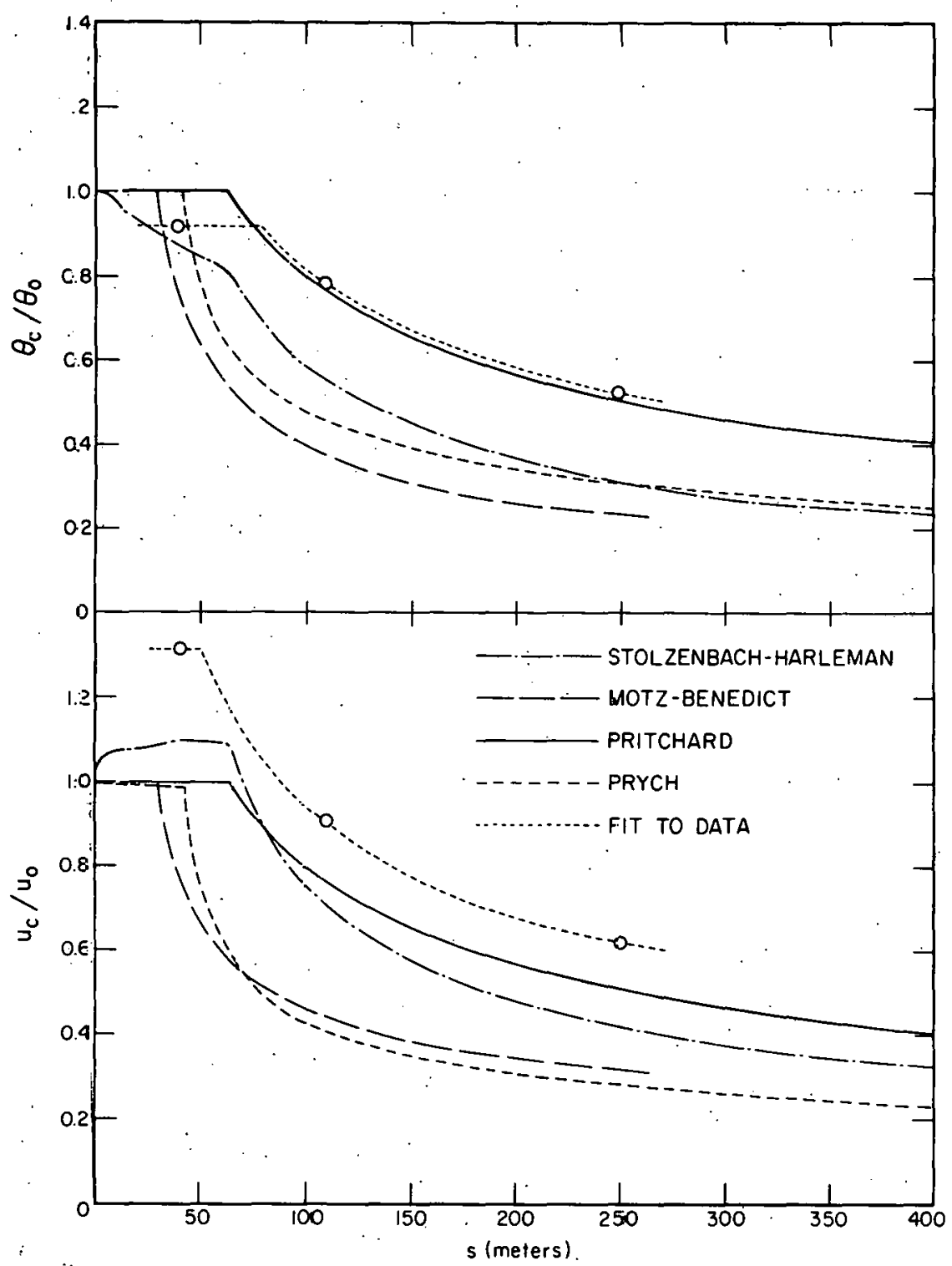

Fig. 65. Centerline Temperature Excess and Velocity Decays Resulting from the Fitting Procedure and Midel Calculations for Point Beach: July 13, 1972. ANL Neg. No. 190-958.

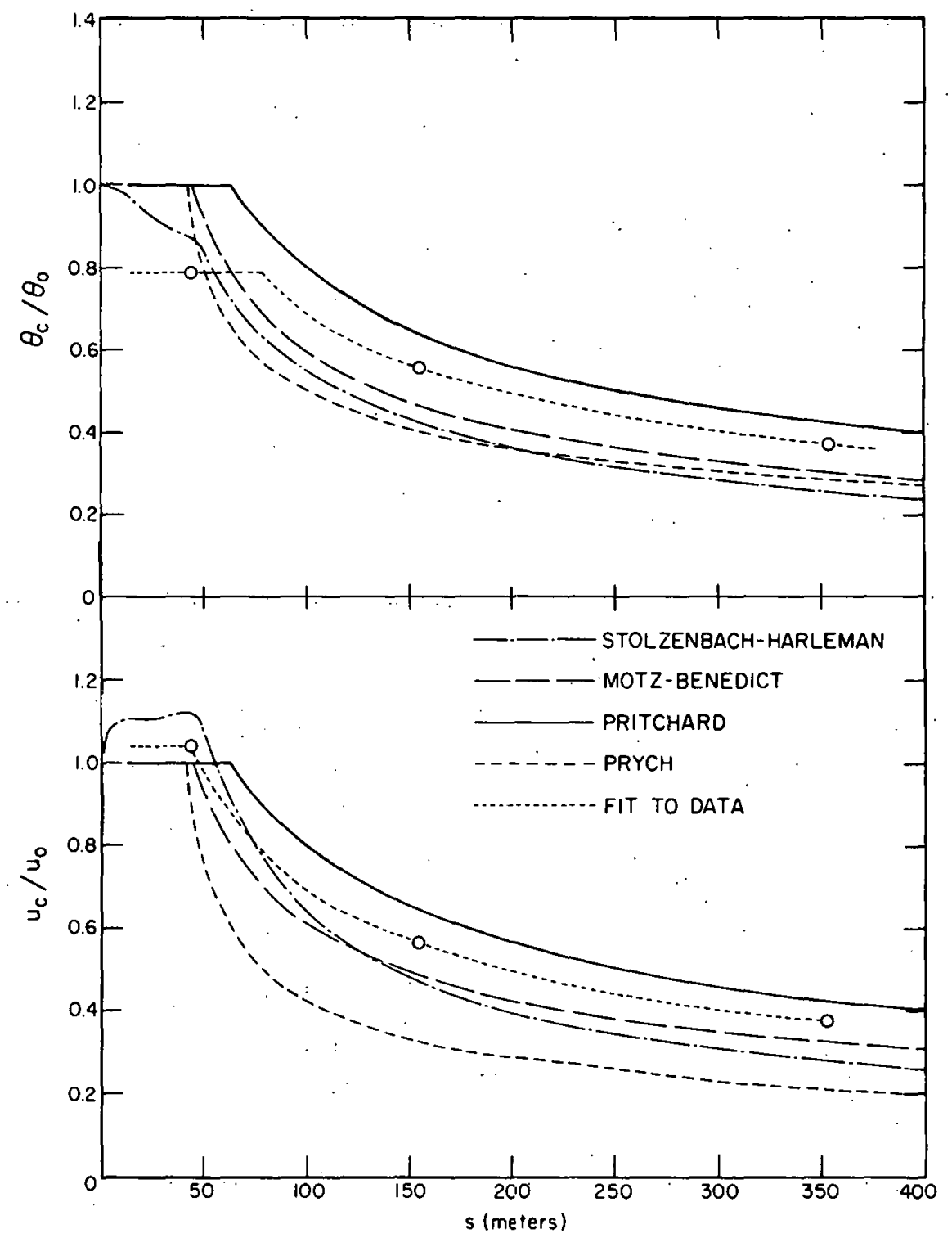

Fig. 66. Centerline Temperature Excess and Velocity Decays Resulting from the Fitting Procedure and Model Calculations for Point Beach: September 9, 1972. ANL Neg. No. 190-951. 


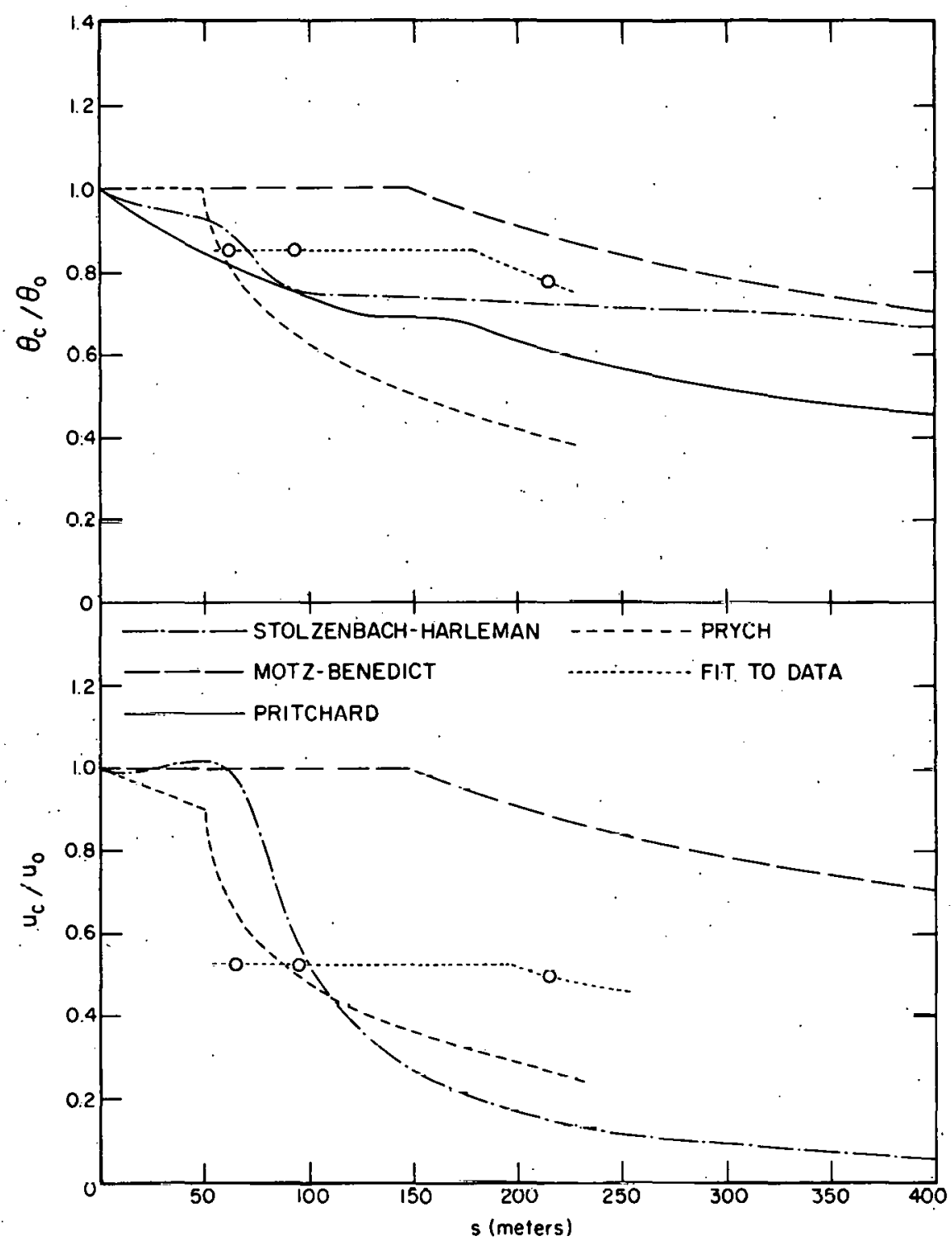

Fig. 67. Centerline Temperature Excess and Velocity Decays Resulting from the Flitung Prucediure and Modcl Calculations for Palisades: October 10, 1972. ANL Neg. No. 190-960.

The Pritchard model appears most accurate and also conservative for the four Point Beach cases of Figs. 63-66. Both JETFIT and the Pritchard model have centerline temperature decay rates proportional to the $-1 / 2$ power of the centerline distance; hence, both curves become parallel as $s$ increases. The Motz-Benedict model gives reasonably conservative predictions for the no-current cases of May 18 and May 23 where the entrainment coefficient was chosen to be 0.05 . (Benedict recommends 0.04 for a $90^{\circ}$ outfall and suggests a slight increase for an off $-90^{\circ}$ discharge.). The model does poorly on July 13 and September 9, apparently due to the choice of entrainment coefficient. The authors' recommendation for the value of the entrainment coefficient for cases with ambient current less than $0.2 u_{0}$ is not clear. The model overpredicts temperature decay for both these cases. The Stolzenbach-Harleman and Prych models both predict a much greater temperature decay than the data indicate. When no current exists, the Stolzenbach-Harleman decay is initially very abrupt, 
yet tends to level off after about $125 \mathrm{~m}$; the centerline temperature data eventually drop below the model results. For the current cases of July 13 and September 9, the centerline decay of Stolzenbach and Harleman is more regular, yet is too rapid. The Prych model predicts too great a temperature decay, yet does so at a rather regular rate of decrease, seemingly independent of an ambient current. Moreover, Prych predicts lower temperatures than Stolzenbach and Harleman, at least within the first 200-250 m from the outfall. Beyond this point, the two models run nearly parallel.

Figures 68-72 show the widths $\mathrm{W}_{\mathrm{T}}$ of the excess temperature distributions resulting from JETFIT and the model calculation. (Note that the linearity of the JETFIT results is a consequence of the linear form assumed in the fitting function.) A very rapid rate of lateral spreading is apparent for the Prych and Stolzenbach-Harleman models. Both models require an assumed form for a lateral spreading velocity that is instrumental in determining the

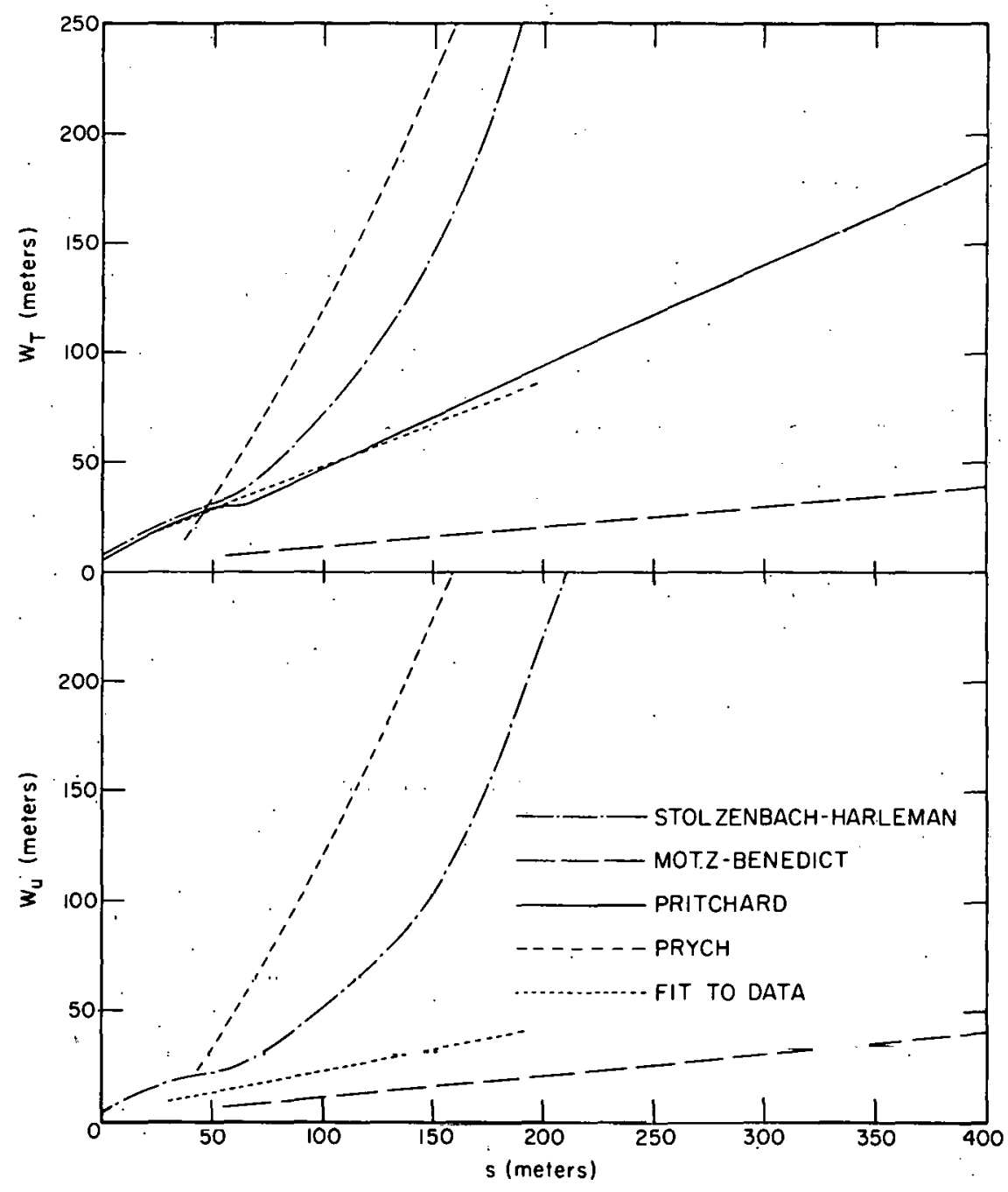

Fig. 68. Half-widths of Temperatire and Velocity Distributions Resulting from the Fitting Frocediue and Mudel Calculations for Point Beàch: May 18, 1972. ANL Neg. No. 190-947. 


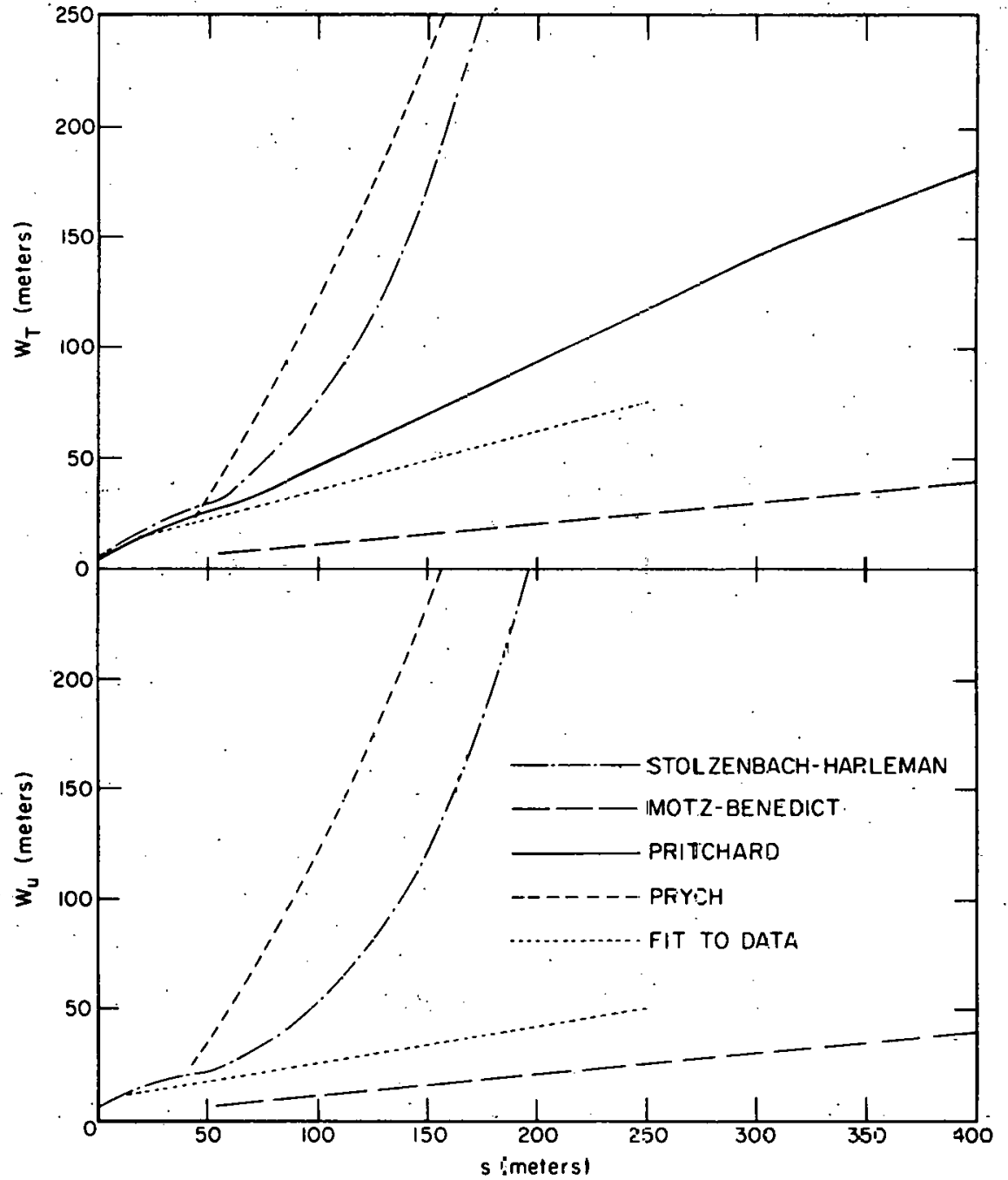

Fig. 69. Half-widths of Tempere ture and Velc.city Distributions Resulting from the Fitting Procedure and Madel Calculations for Point Beach: May 23, 1972. ANミ Neg. NJ. 190-946.

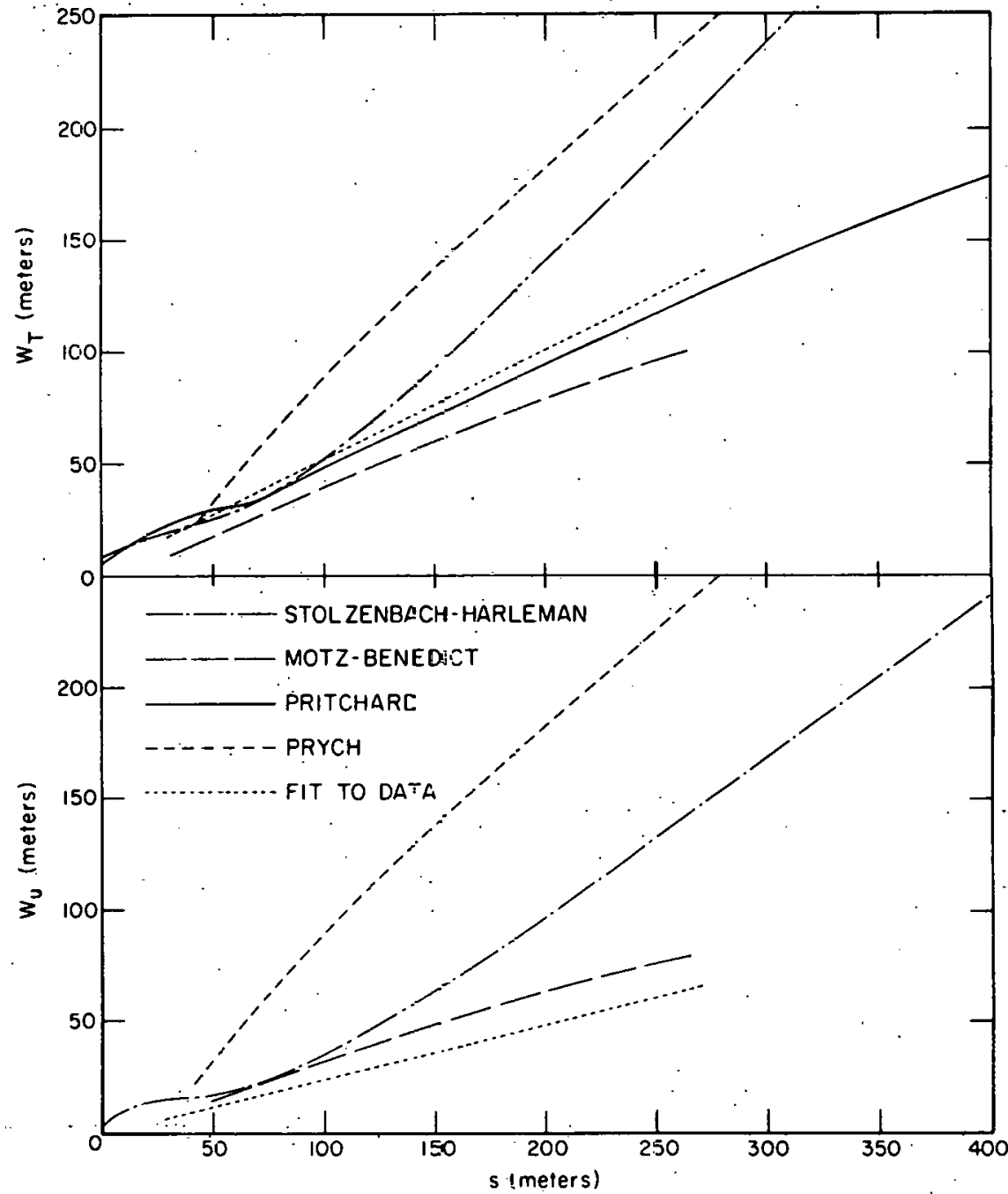

Fig. 70. Half-widths of Ternperature and Velocity Distributions Resulting from the Fitting Frocedure and Model Calculations for Foint Beach: July 13, 1972. ANL Neg. No. 190-942. 


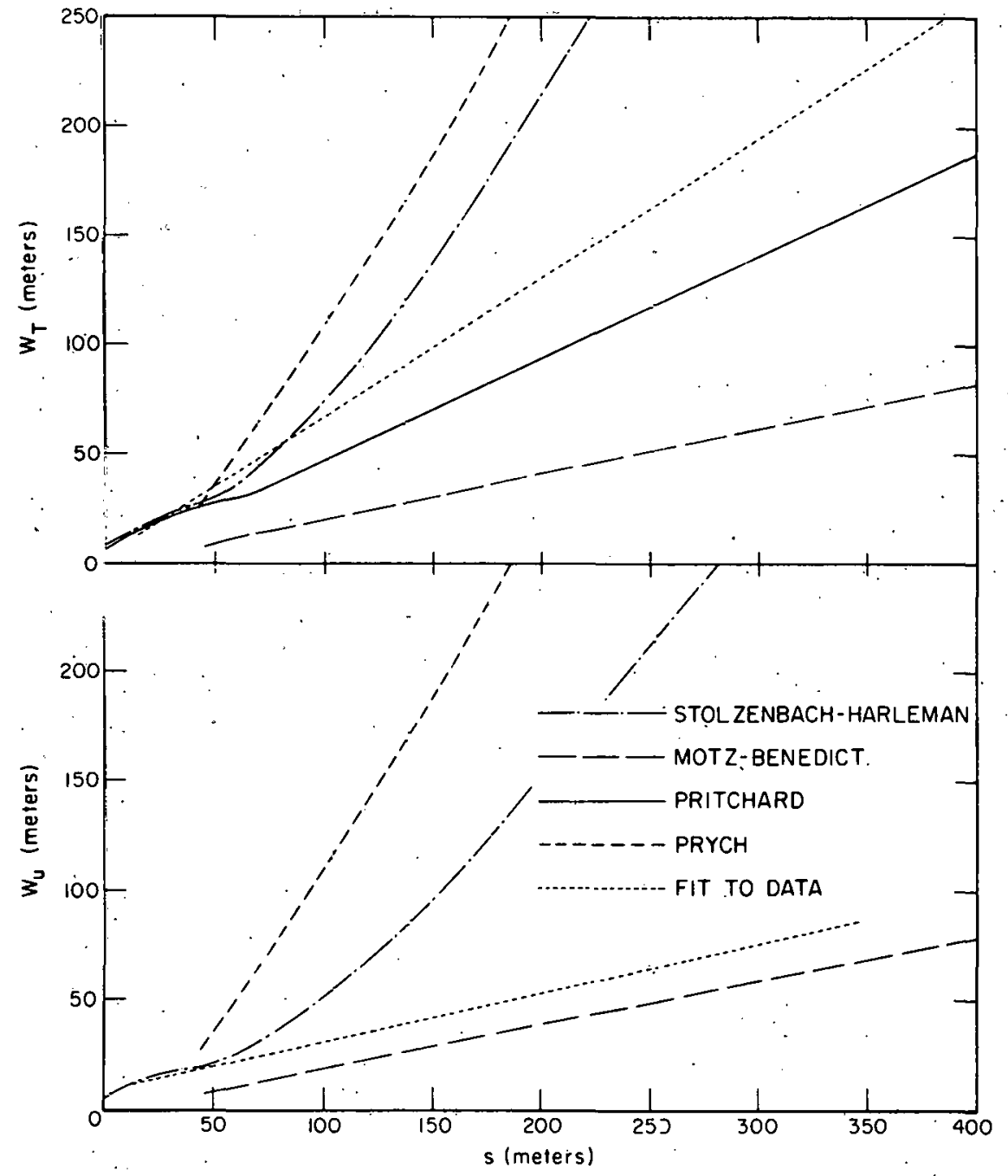

Fig. 71. Half-widths, of Temperature and Velocity Distributions Resulting from the Fitting Procedure and Model Calculations for Point Beach: September 9,1972. ANL Neg. No. 190-941.

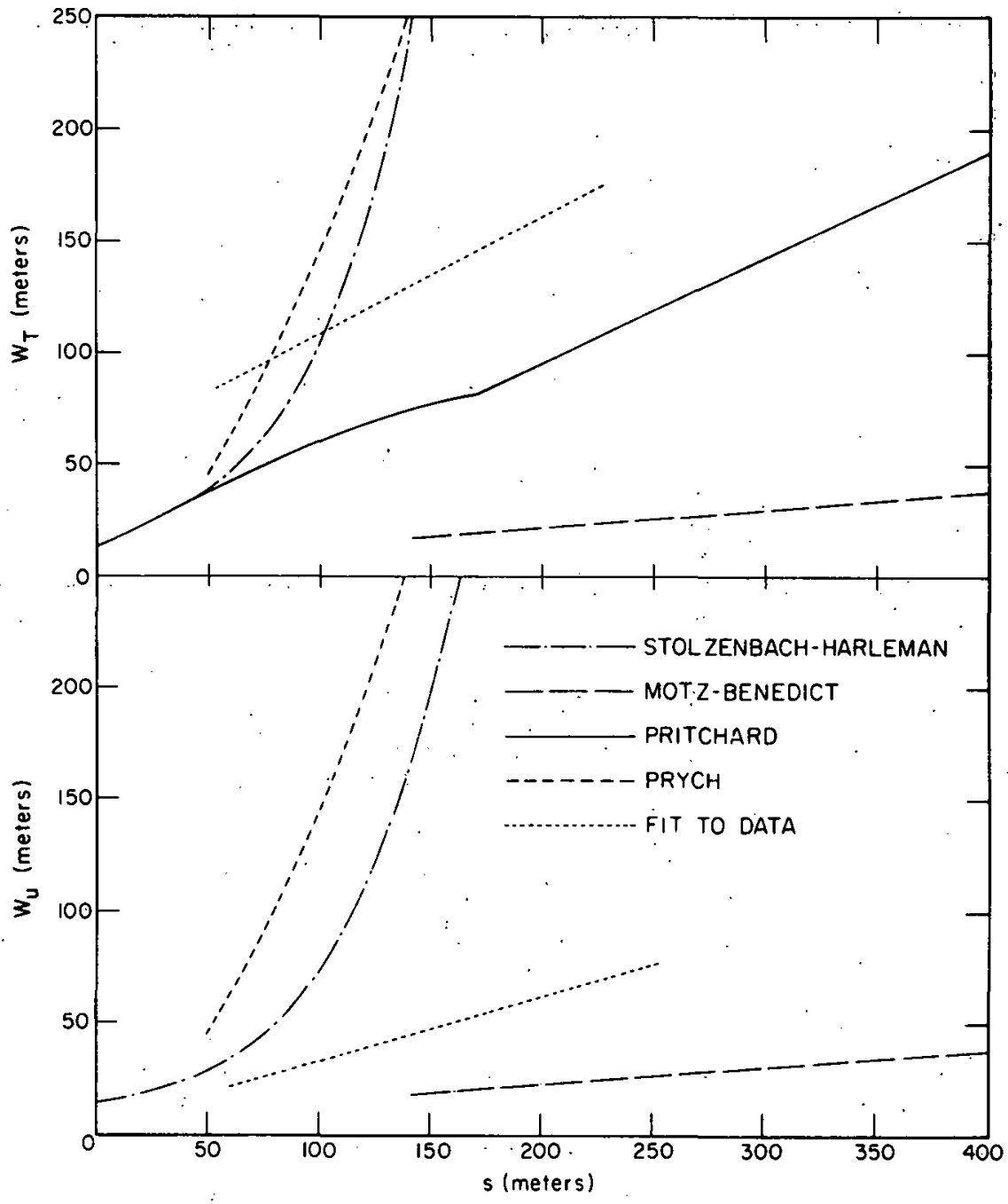

Fig. 72. Half-widths of Temperature and Velocity Distributions Resulting from the Fitting Procedure and Model Calculations for Palisades: October 10, 1972. ANL Neg. No. 190-959. 
lateral extent of the jet. Both models, on the basis of present results, used assumptions that were apparently too theoretical in nature and apparently require calibration with some empirical data to yield more accurate results. The overextended lateral spread of these two jet solutions is a probable cause of the extremely rapid centerline temperature decay required by energy conservation at each jet cross section.

The Motz-Benedict model predicts a jet that is by far too narrow for all four Point Beach cases. The model is very sensitive to the particular choice for the value of some of the input parameters, in particular, the entrainment coefficient and the jet width at the end of the region of flow establishment. A more judicious choice for these parameters would undoubtedly improve the comparisons. However, when this model is used as a predictive tool, it is difficult, at present, to know what values to choose other than those recommended by the authurs of the model.

Pritchard predicts rather accurate temperature decays and widths, except for the temperature width on September 9. The wider width of the data might well be due to the second unit operating at $12 \%$ power on that date, adding to the temperature excesses on the offshore side of the plume.

The Palisades results are quite interesting (Figs. 67 and 72). As expected, the temperature does not drop off until about $175 \mathrm{~m}$ downstream, due to the larger region of flow establishment resulting from the wider outfall at Palisades. Again, Prych and Stolzenbach and Harleman are still too optimistic in their temperature-decay predictions and too wide in their lateral jet spreading. Pritchard predicts too great a temperature decrease and too narrow a jet. This is probably due to his method of handling vertical entrainment, which is very sensitive to the difference between the outfall depth and the critical mixing depth of the lake. Vertical entralnment is not a factor in Pritchard's predictions for Point Beach. Motz and Benedict are conservative in temperature decay for Palisades due to the large region of flow establishment ( 5.2 times the full width of the outfall). Lateral withs are again very much underpredicted.

\section{Centerline Velocity Decay and Velocity Half-widths}

Figures 63-67, for $u_{c} / u_{0}$ derived from JETFIT, indicate a significant deviation of the initial jet velocity from the calculated average channel velocity. For July 13, velocity measurements near the channel outlet indeed verified a velocity greater than $u_{0}$ for the first meter depth. Other measurements, on different dates, all showed values greater than $u_{0}$.

The Stolzenbach-Harleman and Prych models predict too rapid a velocity-centerline decay. In each case, the Stolzenbach-Harleman model predicts an increase in velocity after the jet leaves the outlet up to $60 \mathrm{~m}$ along the centerline. This is possible for discharges having very low densimetric Froude numbers. A light fluid, discharged over a heavier fluid, 
accelerates laterally as well as longitudinally due to hydrostatic pressure gradients. For low densimetric Froude numbers (near 1.0) such buoyant accelerations become dominant. Although increases in velocity are possible, increases in temperature are never realistic. If such hydrostatic pressure gradients were removed or neglected in the model, a momentum jet would occur. For discharges having high densimetric Froude numbers, lateral accelerations greatly dominate longitudinal ones and consequently allows such longitudinal gradients to be neglected. The densimetric Froude numbers for the four Point Beach cases are about 2.4, which may be a little large for the above floating-plume phenomenon to occur. For the stagnantlake cases of Figs. 63 and 64, the Prych model has a sharp decrease in velocity within $125 \mathrm{~m}$ of the outfall and then tends to level off. The

Stolzenbach-Harleman model is more regular in its rapid velocity decline, crossing the Prych curve for $u_{c} / u_{0}$ at about $170 \mathrm{~m}$ from the outfall. The ambient-current cases realize a more regular velocity decline, with the Prych model giving consistently lower velocities.

The Motz-Benedict and Pritchard models appear adequate for both stagnant-lake cases of May 18 and May 23, and the current case of September 9. The data of July 13 reveal higher velocities than predicted by any of the models. An apparent defect in the Motz-Benedict model is the as sumption of decay of lateral velocity to zero, even where an ambient current exists. A more realistic assumption would require a decay superimposed on $U_{a} \cos \beta$ (the component of the ambient current parallel to the local jet centerline).

Velocity widths as predicted by the Prych and Stolzenbach-Harleman models are much too large for the four Point Beach dates (Figs. 68-71). This again reflects on their assumptions of lateral spreading velocity. The Prych. predictions are consistently larger than those of Stolzenbach and Harleman. The Stolzenbach-Harleman predictions appear too sensitive to ambient current; the Prych predictions seem very insensitive. Pritchard has no predic tions for velocity width. Examining the data reveals that the temperature width is approximately twice the size of the velocity width for any fixed date at Point Beach.

The Palisades data indicate a very slow velocity decline after a rather rapid drop in the first $50 \mathrm{~m}$ (see Fig. 67). This rapid drop might be due to the long, diverging discharge channel. Irregularities in the velocity distribution across the channel (lake water entering the channel at the north end combined with significant channel-depth irregularities) may be a contributing factor. No model for velocity decay appears adequate for Palisades on October 10. Velocity widths of Prych and Stolzenbach and.Harleman are again too large, and the Motz-Benedict predictions, although more reasonable, are too small (see Fig. 72). Due to site-dependent irregularities (diverging outfall, shallow bottom), Palisades is not the ideal site to evaluate near-field models, albeit a real site. 
D. Temperature and Velocity Half-depth

Figures 73-76 compare the predictive models with respect to half-depth. The half-depth is the vertical distance at which the excess temperature and/or excess velocity drop to one-half the local surface-centerline value. Also shown is an indication of the location of the lake bottom. No data are plotted, since the vertical profile information was insufficient to define a half-depth in most cases. Either the $3.0-\mathrm{m}$ limit or the lake bottom was reached before the halfdepth or the temperature or velocity distribution was reached.

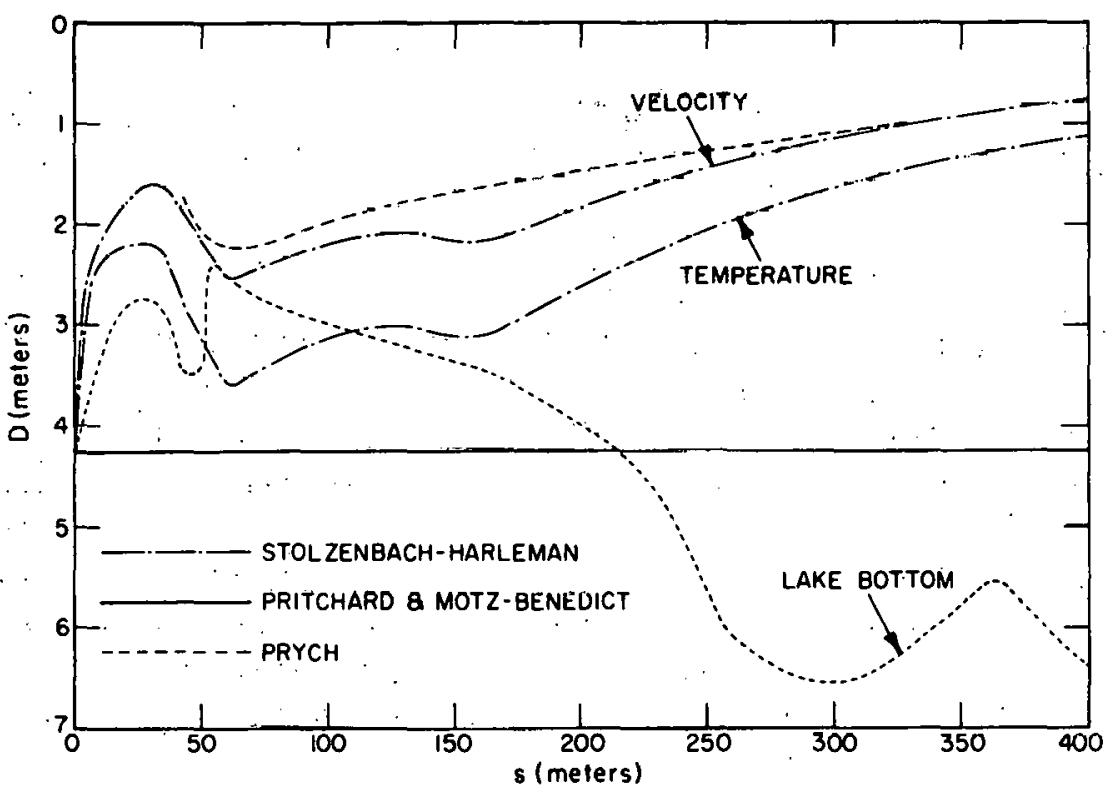

Fig. 73. Half-depths of Temperature and Velocity Distributions Resulting from Model Calculations for Point Beach: May 18, 1972

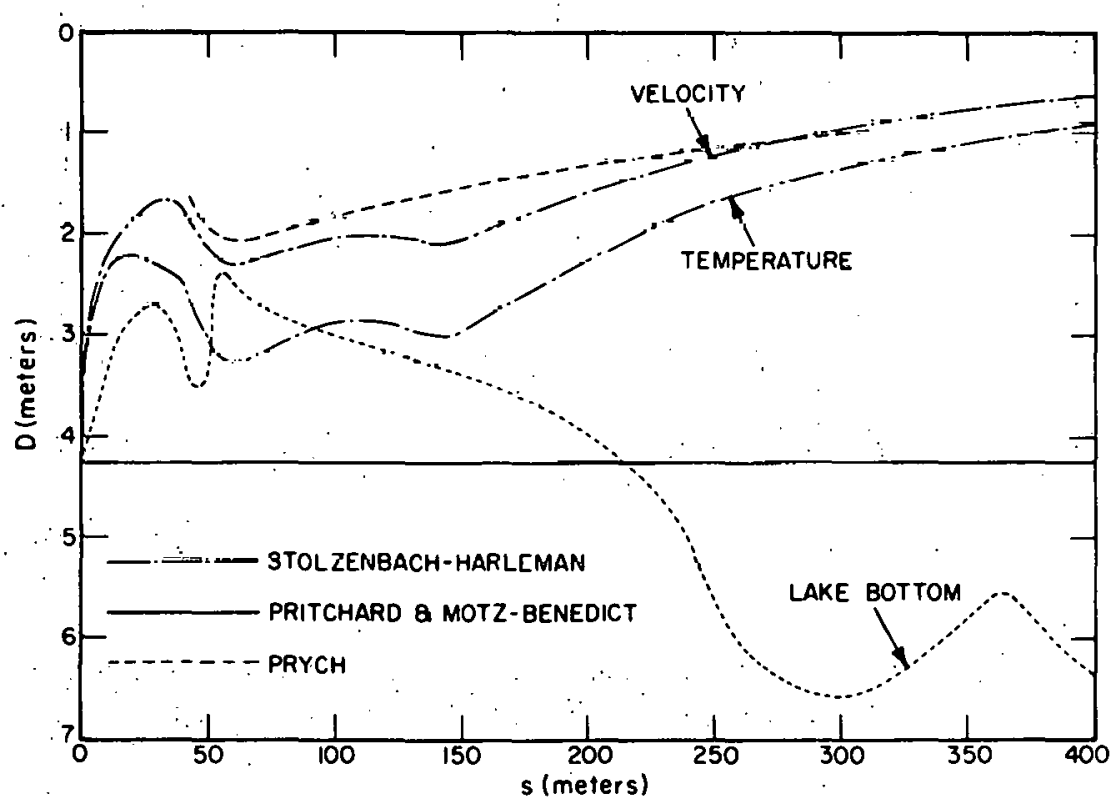

Fig. 74. Half-depths of Temperature and Velocity Distributions Resulting from Model Calculations for Point Beach: May 23, 1972 


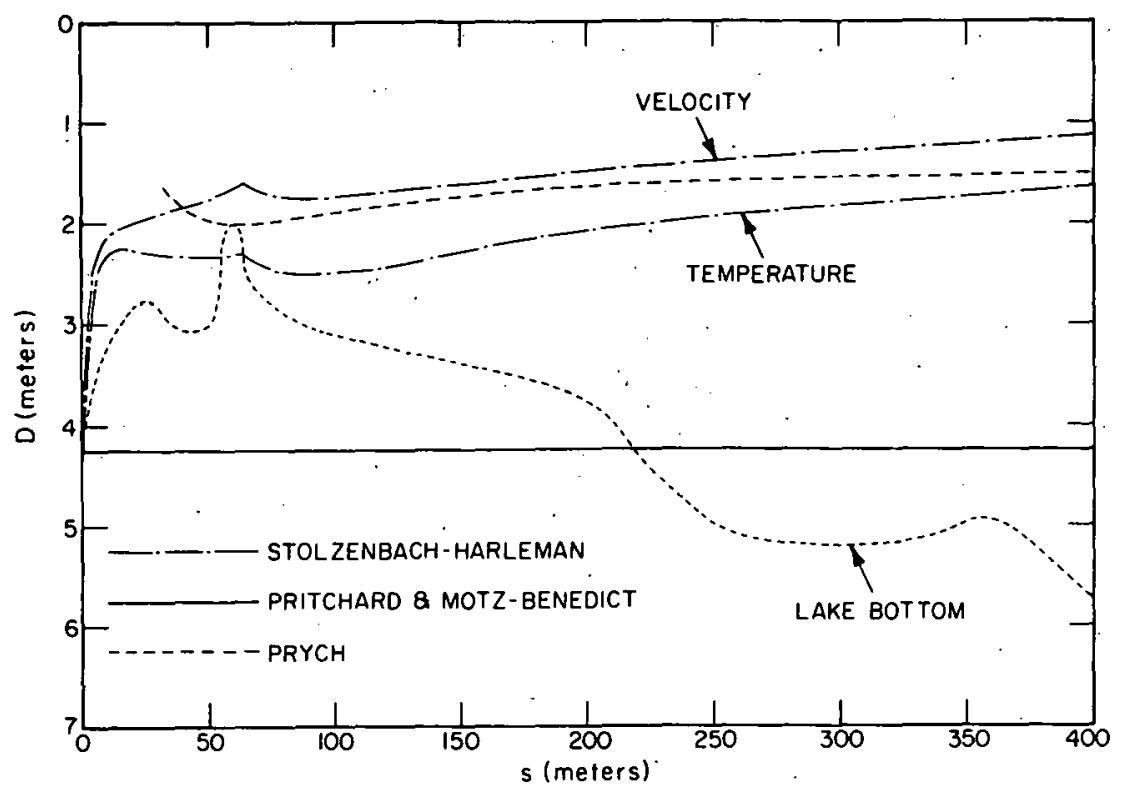

Fig. 75. Half-depths of Temperature and Velocity Distributions Resulting from Model Calculations for Point Beach: July 13, 1972

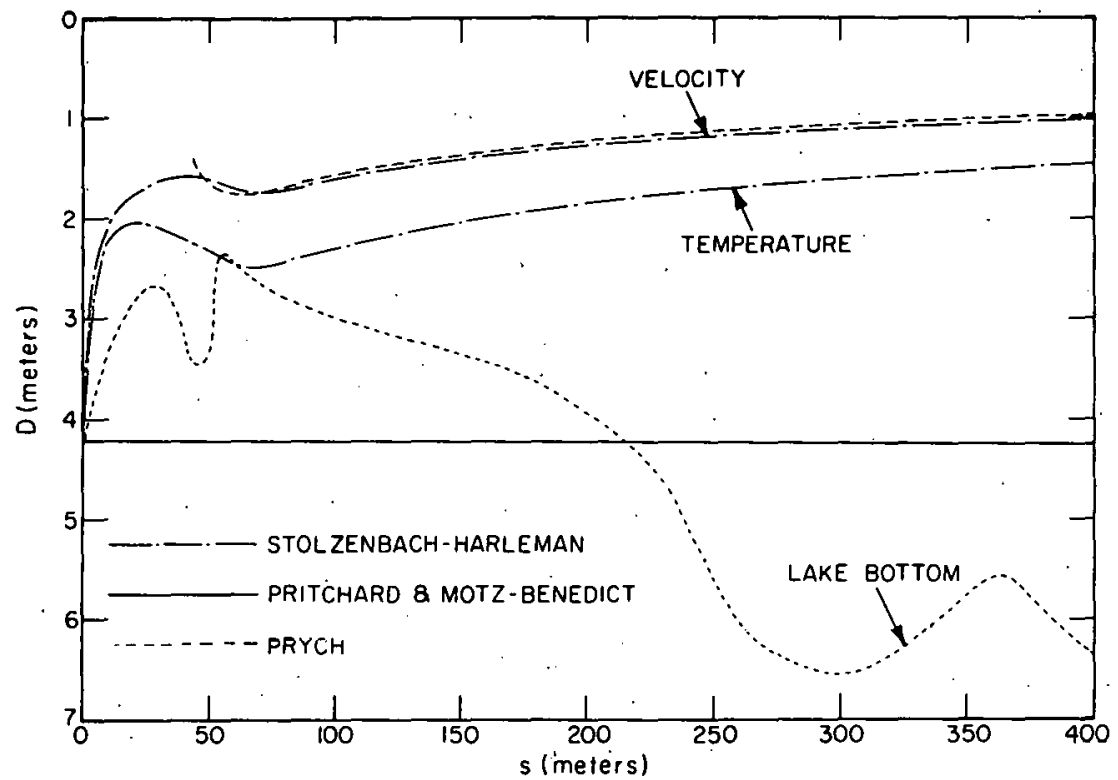

Fig. 76. Half=depthe of Tempcraturc and Velüily. Disiributions Resulting from Model Calculations for Point Beach: September 9, 1972

The Motz-Benedict and Pritchard models, being two-dimensional, naturally predict constant-depth jets. However, Prych and Stolzenbach and Harleman predict a buoyant jet that spreads into a thin layer (typically 1.0 to $1.5 \mathrm{~m}$ thick), after brief contact with the lake bottom for a distance of about $100 \mathrm{~m}$. The Stolzenbach-Harleman model predicts several small ridges in the jet-depth $\mathrm{nn}$ luur that do not appear reasonable. The sharp decrease in jet-depth within the first $75 \mathrm{~m}$ might be associated with the increase in velocity above the channel velocity 
near the outfall predicted by Stolzenbach and Harleman. The Prych model contains no mechanism to allow for a difference in the vertical temperature and velocity spreading; Stolzenbach and Harleman employ different velocity and temperature similarity functions. Note also that Prych consistently predicts thinner plumes for the four Point Beach cases. Bottom interaction, as predicted by the above three-dimensional models, appears less than was actually determined by field measurements. Tables V-IX summarize the vertical distributions of excess temperature and excess velocity on a locationby-location basis for the four dates at Point. Beach and one at Palisades. The jet appears to be influenced by bottom interaction in the vicinity of the outfall, followed by stratification at the final transect. Changes in temperature and velocity excess wilh lepth tend to indicate a lens = shappr plume, with a greater vertical spread of heat than momcntum. Estimates of the plume velocity and temperature half-depths as the plume stratifies are given in the tablut. Al Point Beach, the plume tends to stratify to a temperature depth of $2 \mathrm{~m}$ from an initial temperature depth of $4.2 \mathrm{~m}$; the velocity spreading is to approxi-mately $1.5 \mathrm{~m}$. The Palisades plume is thinner: It reaches an asymptotic temperature and velocity depth of about $0.5-1.5 \mathrm{~m}$ from an initial discharge depth of $2.1 \mathrm{~m}$. The data are not sufficiently refined to permit more than just a gross estimate. Irregularities in the decay of temperature and vclocity excess from an expected monotonic profile are due perhaps to:

1. Different ambient values of temperature and velocity at each depth.

2. The transient thermal-front phenomenon, which complicates the concept of a steady-state temperature at a single-localius.

Thị irregular behavior is real and cannot be ignored; it reflccts on the transient nature of the jet discharge as well as a discharge into a slrulified ambient environrient.

As is evident from the data, bottom interaction should imply greater lateral spread, since vertical entrainment is reduced; even the observed spread was not nearly as great as Prych and Stolzenbach-Harleman predict for no interaction. The bottom influence appears to be most significual fơ i the first $150 \mathrm{~m}$ offshore for the four Point Beach cases with an asymptotic approach to a half-depth of temperature and velocity of $1.0-1.5 \mathrm{~m}$.

Figure 77 compares model predictions for plume half-depth along the jet centerline with actual lake-bottom depths to assist in determining bottom interaction for October 10 at Palisades. Again, each model prediction in Fig. 77 represents the half-depth along the centerline predicted by that model. Predicted model centerlines do not coincide, but are sufficiently close for the present discussion. The lake-bottom depth shown in Fig. 77 is along a line straight out from the outfall.

As can be seen in the figure, the lake depth is about $2 \mathrm{~m}$ to a distance of $125 \mathrm{~m}$ from the outfall; then the bottom drops away rather sharply. The 
TABLE V. DETERMINATION OF TEMPERATURE AND VELOCITY HALF-DEPTHS

AT EACH STATION LOCATION FROM THE POINT BEACH DATA OF MAY 18, 1972

\section{$\underline{S} \underline{T} \underline{A} \underline{T} \underline{I} \underline{O} \underline{N} \underline{N} \underline{U} M \underline{B} \underline{E} \underline{R}$}

Temperature Excess at Each Depih Level Measured

\begin{tabular}{|c|c|c|c|c|c|c|c|c|c|c|c|c|c|c|c|c|c|}
\hline $0.5 \mathrm{~m}$ & 1.8 & 2.9 & $\mathrm{NM}$ & 4.8 & 5.3 & 1.9 & 0.0 & 4.1 & 5.3 & 3.8 & 1.3 & 1.4 & 1.8 & 7.5 & 6.8 & 6.8 & 4.6 \\
\hline $1.0 \mathrm{~m}$ & 1.2 & 2.5 & 4.1 & 5.5 & 5.0 & 2.1 & LB & 4.6 & 5.9 & 5.4 & 1.2 & 0.0 & 1.8 & 7.5 & 6.0 & 6.2 & 5.1 \\
\hline $1.5 \mathrm{~m}$ & $\mathrm{NM}$ & 2.1 & $N M$ & 4.9 & 4.3 & 1.4 & LB & 3.8 & 6.7 & 5.1 & $\mathrm{MM}$ & 0.0 & 0.1 & 7.9 & 6.7 & 6.1 & 4.9 \\
\hline $2.0 \mathrm{~m}$ & 2.2 & $0 . .5$ & 3.1 & 2.7 & 4.8 & 1.5 & $L B$ & LB & 6.8 & 3.8 & 0.6 & $L B$ & LB & 7.3 & 6.5 & 5.6 & 4.7 \\
\hline $2.5 \mathrm{~m}$ & $N M$ & 0.2 & 1.4 & 4.4 & 3.5 & 1.2 & LB & $L B$ & 5.6 & 2.4 & LB & LB & $\mathrm{LB}$ & $L B$ & $L B$ & $\mathrm{LB}$ & 5.2 \\
\hline $3.0 \mathrm{~m}$ & $\mathrm{LB}$ & LB & $\mathrm{NM}$ & $\mathrm{NM}$ & $M M$ & $\mathrm{NM}$ & LB & LB & LB & LB & $\mathrm{LB}$ & $L B$ & $L B$ & LB & LB & $L B$ & LB \\
\hline
\end{tabular}

\section{Velocity Excess at Each Depth Level Measured}

\begin{tabular}{|c|c|c|c|c|c|c|c|c|c|c|c|c|c|c|c|c|c|}
\hline $0.5 \mathrm{~m}$ & 0.0 & 0.0 & $\mathrm{NM}$ & 17.9 & 26.1 & 15.5 & 0.0 & 0.0 & 32.3 & 37.3 & 0.0 & 0.0 & 9.5 & 56.3 & 29.4 & 17.8 & 7.4 \\
\hline $1.0 \mathrm{~m}$ & 0.0 & 0.0 & 2.8 & 7.6 & 21.8 & 7.8 & $\mathrm{LB}$ & 0.0 & 33.0 & 37.6 & 0.0 & 0.0 & 7.9 & 47.1 & 30.2 & 20.2 & 8.8 \\
\hline $1.5 \mathrm{~m}$ & $\mathrm{NM}$ & 2.5 &.$M$ & 6.5 & 23.9 & 0.0 & $\mathrm{LB}$ & 0.0 & 29.7 & 28.8 & $\mathrm{MM}$ & 0.0 & 1.6 & 48.3 & 21.5 & 15.0 & 5.1 \\
\hline $2.0 \mathrm{~m}$ & 0.0 & 2.5 & 0.0 & 4.4 & 19.4 & 0.0 & $L B$ & LB & 21.7 & 20.1 & 0.0 & $\mathbf{L B}$ & $\mathrm{LB}$ & 48.4 & 26.2 & 9.4 & 0.0 \\
\hline $2.5 \mathrm{~m}$ & $\mathrm{NM}$ & 5.2 & 0.0 & $0.0^{\circ}$ & 15.4 & 0.0 & $\lfloor B$ & $L B$ & 16.5 & 12.4 & LB & $\mathrm{LB}$ & $L B$ & $\mathrm{LB}$ & $\mathrm{LB}$ & LB & 4.4 \\
\hline $3 . \therefore \mathrm{m}$ & $L B$ & LF: & $\mathrm{PM}$ & MM & $N M$ & - $N M$ & $\mathrm{LB}$ & LB & $\mathrm{LB}$ & LB & $L B$ & $\mathrm{LB}$ & $\mathrm{LB}$ & LB & LB & LB & $\mathrm{LB}$ \\
\hline
\end{tabular}

LB = LAKE BOTTOM INITERTERENCE MM $=$ NOT MEASURED

POINTS OF IRREGULAR IERTICAL EXCESS-TEMPERATLRE VARIATION: $1,4-6,8-10$, and 14-16.

FROM POINTS $2,3,7,11-13$, THE TEMPERATURE FALF-DEPTH OF THE' JET MAY BE ESTIMATED TO BE ABOUT 2 METERS.

POINTS OF IRREGULAR IERTICAL EXCESS-VELOCITY VARIATION: $2,5,9,14,15,16$, AND 17.

FROM POINTS $1,3,4,6-8,10-13$, AND 18, THE VELOCITY HALF-DEPTH OF THE JET MAY BE ESTDMATED AS 1.5 METERS. 


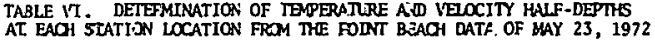

Temperature Excess at Each Depth Lerel Measured

\begin{tabular}{|c|c|c|c|c|c|c|c|c|c|c|c|c|c|c|c|c|c|c|c|c|c|c|c|c|c|c|c|c|}
\hline \multirow{2}{*}{$\begin{array}{l}0.5 \mathrm{a} \\
1.0 \mathrm{~g}\end{array}$} & 0.6 & 4.5 & 6.5 & 6.8 & 6.6 & 6.2 & 2.2 & 5.1 & 1.3 & 6.4 & 5.j & 3.1 & 1.3 & 5.5 & 5.0 & 5.8 & 3.2 & 0.2 & 2.4 & 4.3 & 5.5 & 2.0 & 2.3 & 0.0 & 2.3 & 3.0 & 4.0 & 2.3 \\
\hline & 2.2 & 6.2 & 8.2 & 8.4 & 8.3 & $7 .:$ & 1.2 & $\cdot .0$ & 2.5 & 8.0 & 7.0 & 3.9 & 3.0 & 5.6 & 7.2 & 0.6 & $4.4^{\circ}$ & 1.4 & 3.5 & 6.0 & $5.6 i$ & 2.2 & 2.4 & 0.0 & 2.7 & 5.0 & 4.2 & 3.1 \\
\hline $1.5 \mathrm{~m}$ & 2.5 & 5.1 & 8.7 & 9.0 & 8.9 & $7 .:$ & 0.6 & $\therefore .7$ & 2.2 & 7.6 & s.? & 3.8 & 2.6 & 5.6 & 6.5 & 6.6 & 2.8 & 1.6 & 2.9 & 5.7 & 3.7 & 1.7 & 1.7 & 1.4 & 2.6 & 5.0 & 3.7 & 2.8 \\
\hline $2.0 \mathrm{~m}$ & LB & 5.5 & 9.3 & 9.5 & 9.4 & $6 . \varepsilon$ & 0.9 & $\because 5^{\prime}$ & LB & 7.9 & 7.9 & 3.7 & 2.8 & 5.7 & 7.4 & 7.1 & 2.7 & 2.1 & 2.4 & $6: 0$ & 3.5 & 1.7. & 1.2 & 0.0 & 2.5 & 4.0 & 3.1 & 1.2 \\
\hline $2.5 \mathrm{~m}$ & LB & 5.5 & 9.3 & 9.7 & 9.7 & 6.2 & 1.1 & LB & LB & 8.0 & 6.0 & 4.0 & 2.8 & LB. & M & 6.3 & 1.0 & $L B$ & LB & 6.0 & 5.3 & 0.3 & 0.0 & 0.0 & 2.0 & 4.0 . & 3.4 & 1.5 \\
\hline $3.0 \mathrm{~m}$ & LBB & LB & $8: 5$ & 10.1. & 10.0 & $5 . \epsilon$ & LB & LB & LB & LB & $M$ & -B & LB & LB & $\mathbf{M}$ & M & M & $\dot{L B}$ & LB & 5.5 & 3.3 & 0.4 & 0.8 & 0.0 & 1.3 & 4.0 & 3.5 & 2.0 \\
\hline
\end{tabular}

$\begin{array}{lllllllllll}3.0 \mathrm{~m} & \text { LB } & \text { L.B } & 8.5 & 10.1 & 10.0\end{array}$

Velocity Excess at Each Depth Level Measured

\begin{tabular}{|c|c|c|c|c|c|c|c|c|c|c|c|c|c|c|c|c|c|c|c|c|c|c|c|c|c|c|c|c|c|}
\hline $0.5 \mathrm{~m}$ & 1.5 & 11.9 & 61.0 & 66.2 . & 72.6 & 45.6 & $\mathrm{i} 2.6$ & 9.5 & 13.3 & 66.7 & 55.5 & 170 & 0.0 & .40 .2 & 48.6 & 41.8 & 18.2 & -4.2 & 5.2 & 22.2 & 5.3 & 20.1 & 10.1 & 0.0 & 15.5 & 18.0 & 21.0 & 2.2 & 1.3 \\
\hline $1.0 \mathrm{~m}$ & 0.0 & $14: 2$ & 54.1 & 59.8 & 59.3 & 48.4 & 5.9 & $\therefore .4$ & 13.2 & 69.5 & 36.0 & 11.8 & 2.1 & 34.5 & 40.5 & 41.1 & 13.8 & -6.4 & 0.0 & $=4.5$ & 35.3 & 14.5 & 0.0 & 0.0 & 0.1 & 16.3 & .13 .9 & 0.0 & 2.5 \\
\hline $1.5 \mathrm{~m}$ & 0.0 & 36.9 & 48.5 & 58.8 & 58.4 & 40.7 & 3.6 & $\therefore 0$ & $-\dot{5} .8$ & 47.3 & 22.7 & 5.6 & -1.6 & $2^{-} .2$ & 50.0 & 43.2 & 5.2 & -5.2 & 0.0 & \pm 0.6 & $2 \because 1$ & 6.6 & 0.0 & 0.0 & 8.2 & 10.0 & 9.5 & 0.0 & 0.0 \\
\hline $2.0 \mathrm{~m}$ & LB & 12.1 & 53.9 & 58.2 & 65.6 & 30.3 & 3.1 & 18.7 & LB & 40.0 & $31 . c$ & 5.7 & -3.3 & $\mathrm{r}^{-} .6$ & 27.3 & 35.5 & 0.0 & -3.3 & -2.4 & 3.1 & $2 t .0$ & 1.5 & 0.0 & 0.0 & 0.0 & 5.0 & 0.0 & 0.0 & .0 \\
\hline $2.5 \mathrm{~m}$ & LB & 12.9 & 38.2 & 39.9 & 66.0 & 28.5 & 3.4 & eB & LB & 44.4 & 22.4 & 7.6 & -3.5 & LB & M & 24.2 & 0.0 & LB & LB & 3.3 & $14 . \mathrm{s}_{\mathrm{H}}$ & -0.6 & -3.2 & 0.0 & 0.0 & 0.0 & 0.0 & 0.0 & 0 \\
\hline $3.0 \mathrm{~m}$ & $L B$ & 18 & 43.5 & $49 . i$ & 43.8 & 11.7 & LB & $\mathbf{L B}$ & LB & LB & $N$ & LB & LB & $L B$ & M & M & M & LB & LB & 6.3 & $14.3-$ & 0.3 & -3.1 & 0.0 & 0.0 & 0.0 & 0.0 & 0.0 & 0.0 \\
\hline
\end{tabular}

$3.0 \mathrm{~m} \quad$ LB $L \begin{array}{llllllllllll}43.5 & 49.1 & 43.8 & 11.7 & \text { LB } & \text { LB } & \text { LB } & \text { LB } & \text { NM } & \text { LB } & \text { LB }\end{array}$

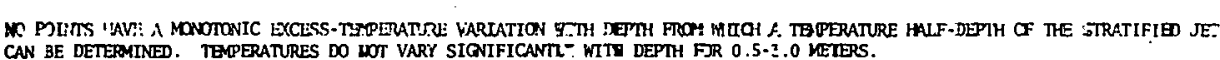

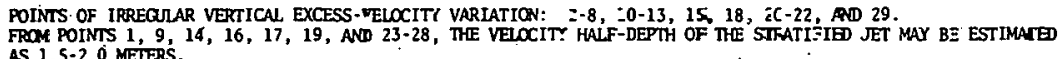


TABLE VII. DETERMTNATION OF TEMPERATURE AND VELOCITY HALF-DEPTHS

AT EACH STATION LOCATION FROM THE POINT BEACH DATA OF JUY 13,1972

\section{$\underline{\mathrm{S}} \underline{\mathrm{T}} \underline{\mathrm{A}} \underline{\mathrm{T}} \underline{\mathrm{I}} \underline{\mathrm{O}} \underline{\mathrm{N}} \underline{\mathrm{N}} \underline{\mathrm{U}} \underline{\mathrm{M}} \underline{\mathrm{B}} \underline{\mathrm{E}} \underline{\mathrm{R}}$}

$\begin{array}{lllllllllllllllll}1 . & 2 & 3 & 4 & 5 & 6 & 7 & 8 & 9 & 10 & 11 & 12 & 13 & 14 & 15 & 16 & 17\end{array}$

Temperature Excess at Each Depth Level Measured

\begin{tabular}{|c|c|c|c|c|c|c|c|c|c|c|c|c|c|c|c|c|c|}
\hline $0.5 \cdot \mathrm{m}$ & $4.0^{\circ}$ & 6.0 & 7.2 & 5.5 & $3.7^{\prime}$ & 3.5 & 3.7 & 5.8 & 6.2 & 4.0 & 4.0 & 2.3 & 3.3 & 3.5 & 3.0 & 2.5 & 2.7 \\
\hline $1.0 \mathrm{~m}$ & 4.5 & 6.8: & 7.8 & 5.8 & 4.5 & 3.8 & 3.3 & 6.1 & 6.1 & 4.1 & 4.0 & 1.8 & 2.8 & 2.8 & 2.5 & $1: 8$ & 1.5 \\
\hline $1.5 \mathrm{~m}$ & 4.6 & 5.6 & 7.1 & 7.1 & 4.6 & 4.1 & 3.8 & 6.3 & 6.9 & 4.6 & 4.3 & 1.1 & 3.1 & 2.6 & 2.1 & 1.3 & 1.1 \\
\hline $2.0 \mathrm{~m}$ & 3.5 & 7.5 & $8 . .5$ & 6.5 & $\mathrm{LB}$ & 3.3 & 2.5 & 6.7 & 5.5 & 4.0 & LB & 0.5 & 3.0 & 2.3 & 1.3 & 0.7 & 0.5 \\
\hline $.5 \mathrm{~m}$ & 2.1 & 4.4 & 8.4 & $\mathrm{LB}$ & $\mathrm{LB}$ & 1.4 & 1.4 & 7.1 & 4.9 & 1.1 & LB & 0.7 & 1.9 & 1.4 & 0.7 & 0.4 & $0: 1$ \\
\hline
\end{tabular}

Velocity Excéss at Each Depth Level Measured.

$\begin{array}{rrrrrrrrrrrrrrrrrrrrr}0.5 \mathrm{~m} & -0.3 & 57.9^{-} & 64.5 & 45.2 & 11.7 & 1.2 & 25.1 & 41.1 & 47.0 & 17.9 & 4.0 & 2.7 & 22.0 & 25.0 & 15.6 & 1.5 & 4.7 \\ 1.0 \mathrm{~m} & 8.5 & 58.0 & 56.6 & 31.9 & 9.3 & -4.8 & 17.0 & 38.8 & 36.1 & 15.6 & -0.8 & -1.3 & 12.5 & 17.7 & 8.8 & -3.6 & 4.4 . \\ 1.5 \mathrm{~m} & 4.6 & 28.3 & 64.8 & 36.7 & -1.3 & -3.1 & 11.8 & 33.5 & 33.4 & 7.5 & -6.5 & 1.5 & 6.8 & 8.3 & 2.9 & -3.3 & 1.1 . \\ 2.0 \mathrm{~m} & 1.8 & 36.9 & 74.5 & 26.9 & \text { LB } & -2.4 & 1.5 & 30.0 & 23.0 & 0.1 & \text { LB } & -2.2 & 3.5 & 4.1 & -3.1 & 7.9 & -3.6 \\ 2.5 \mathrm{~m} & -0.4 & 17.7 & 48.0 & \text { LB } & \text { LB } & -1.4 & -4.8 & 28.5 & 5.5 & 0.6 & \text { LB } & -2.6 & -2.3 & -3.3 & -3.9 & -6.7 & -3.3\end{array}$

LB = LAKE BOTTOM INTERFERENCE

POINTS OF IRREGULAR VERTICAL EXCESS-TEMPERATURE VARIATION: 1-11 and 13.

FROM POINTS 12 AND 14-17, ZHE TEMPERATURE HALF-DEPTH OF THE STRATIFIED JET MAY BE ESTIMATED TO BE ABOUT 2.0-2.5 METERS.

POINTS OF IRREGULAR. VERTICAL EXCESS-VELOCITY VARIATION: $1-4,6,12,16$, and 17

FROM POINTS $5,7-11$, AND $15-15$, THE VELOCITY HALF-DEPTH OF THE STRATIFIED JET MAY BE ESTIMATED AS $1.0-1.5$ METERS. 
TAELE VIII. DETERMINATION OF TEMPERATURE AND VELOCITY HALF-DEPTHS

AT EACH STATION LOCATION FROM THE POINT BEACH DATA OF SEPTEMBER 9, 1972

\section{S T A T I O N N N U M B E R}

$\begin{array}{lllllllllllllllllll}1 & 2 & 3 & 4 & 5 & .6 & 7 & 8 & 9 & 10 & 11 & 22 & 13 & 14 & 15 & 16 & 17\end{array}$

Temperature Excess at Each Depth Level Measured

\begin{tabular}{|c|c|c|c|c|c|c|c|c|c|c|c|c|c|c|c|c|c|}
\hline $0.5 \mathrm{~m}$ & 0.9 & 3.3 & 5.7 & 7.5 & 4.7 & 2.8 & 2.5 & 4.2 & 4.4 & .3 .9 & 3.8 & 3.7 & 3.0 & 3.2 & 3.6 & 3.3 & 2.6 \\
\hline $1.0 \mathrm{~m}$ & .1 .1 & 4.3 & 7.6 & 6.3 & 5.3 & 3.4 & 3.1 & 4.7 & 4.0 & 4.6 & 4.3 & 3.3 & 2.6 & 2.5 & 3.0 & 2.9 & 3.7 \\
\hline $1.5 \mathrm{~m}$ & LB & 5.2 & 5.9 & 7.8 & 3.7 & 4.0 & 3.3 & $4.4^{\circ}$ & 4.4 & 3.5 & 3.9 & 2.4 & 1.6 & 1.5 & 1.4 & 2.4 & 3.9 \\
\hline $2.0 \mathrm{~m}$ & $L B$ & LB & 7.7 & 7.5 & 3.5 & 2.6 & 2.5 & 3.8 & 3.5 & 3.6 & 2.6 & 2.0 & 1.4 & 0.7 & 0.7 & 1.6 & 2.0 \\
\hline $2.5 \mathrm{~m}$ & $L B$ & LB & LB & $\mathrm{LB}$ & LB & LB & $1: 7$ & 1.7 & 2.3 & 2.3 & 1.2 & 0.7 & 1.0 & 0.7 & 0.7 & 0.7 & 1.2 \\
\hline
\end{tabular}

Velocity Excess at Each Depth _evel Measurec

\begin{tabular}{|c|c|c|c|c|c|c|c|c|c|c|c|c|c|c|c|c|c|}
\hline $0.5 \mathrm{~m}$ & -5.9 & 21.8 & 54.5 & 52.0 & 39.7 & 9.6 & 2.8 & 25.7 & 23.6 & 25.0 & 11.7 & 1.2 & 10.5 & 12.4 & 9.8 & 5.2 & -0.5 \\
\hline $1.0 \mathrm{~m}$ & -3.2 & 12.9 & 45.2 & 38.0 & 25.3 & 1.8 & 1.4 & 22.3 & 18.1 & 16.1 & 13.0 & -1.2 & 1.1 & 0.1 & 3.5 . & 3.4 & -0.1 \\
\hline $1.5 \mathrm{~m}$ & LB & 6.9 & 410.4 & 40.3 & $25 . J$ & -5.0 & -0.4 & 13.1 & 12.8 & 4.4 & 6.3 & -2.7 & $-(1.2$ & -0.2 & -0.7 & -0.5 & -0.4 \\
\hline 2.0 & LB & $\mathrm{LB}$ & 43.0 & 43.0 & 13.2 & -6.7 & 0.5 & 0.9 & -0.2 & 7.0 & -0.4 & -3.8 & -0.3 & -0.3 & -0.7 & -0.4 & -1.0 \\
\hline $.5 \mathrm{~m}$ & LB & LB & LB & LB & LB & LB & 0.4 & 0.2 & -0.7 & -1.2 & -5.7 & -2.1 & -0.2 & -0.2 & -1.6 & -4.5 & -2.7 \\
\hline
\end{tabular}

LB $=$ LAKE EOTTOM INTERFERENCE

POINTS OF IRREGULAR VERTICAL EXCESS-TEMPERATURE : VARIATION: :-11 AND 17.

FROM POINTS $12-16$, THE TEMPERATURE HALF-DEPTH OF THE STRATIF IED JET MAYY BE ESTIMATED TO BE ABOUT 2.0 ME.ERS.

POINTS OF IRREGULAR VERTICAL EXCESS-VELOCITY VARIATION: $1,7: 10,11,12$, and 17.

FROM POINTS $2 \div 6,8 \div 9$, AND 13-15, T-EE VELOCITY HALF-DEPTH OF TIE STRATIFIED JET MAY .BE ESTTMATED AS 1.0 ME.E?: 
TAB:E IX. DEIERMINATION OF TEMPERATURE AND VELOCITY HALF-DEPTHS

AT EACH STATION LOCATION FROM THE PALISADES DATA OF OCTOBER 10, 1972

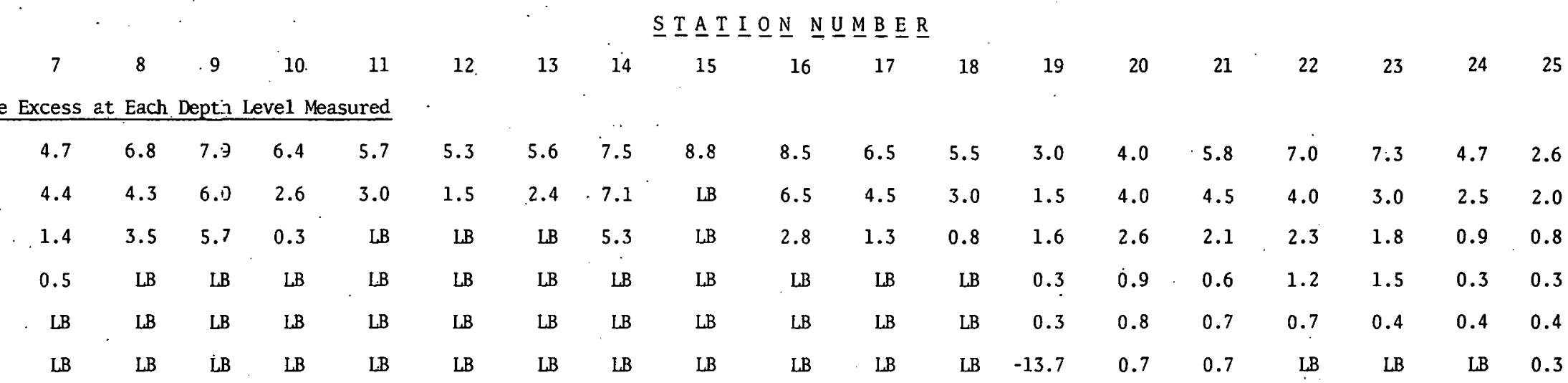

Velocity Excess at Each Depth Level Measured

$\begin{array}{rrrrrrrrrrrrrrrrrrrrr}0.5 \mathrm{~m} & 0.0 & 14.8 & 19.5 & -0.7 & 0.4 & -4.7 & -2.3 & 23.5 & 14.2 & 25.0 & 2.3 & -1.8 & 1.1 & 9.1 & -1.4 & 12.8 & 18.5 & 11.1 & 5.6 \\ 1.0 \mathrm{~m} & 0.0 & 13.7 & 6.9 & -2.1 & -5.1 & -3.9 & -2.9 & 19.2 & \text { LB } & 12.2 & 0.0 & -1.5 & 2.6 & 7.8 & 11.0 & 7.1 & 2.6 & 1.2 & -2.8 \\ 1.5 \mathrm{~m} & -2.2 & 4.0 & 0.0 & -2.8 & \text { LB } & \text { LB } & \text { LB } & 7.6 & \text { LB } & 5.8 & -10.7 & -1.7 & 0.8 & 1.7 & 2.8 & 1.8 & -0.5 & -3.5 & -0.5 \\ 2.0 \mathrm{~m} & -2.3 & \text { LB } & \text { LB } & \text { LB } & \text { LB } & \text { LB } & \text { LB } & \text { LB } & \text { LB } & \text { LB } & \text { LB } & \text { LB } & 3.5 & 1.0 & 2.8 & -1.2 & -5.5 & -3.2 & -2.2 \\ 2.5 \mathrm{~m} & \text { LB } & \text { LB } & \text { LB } & \text { LB } & \text { LB } & \text { LB } & \text { LB } & \text { LB } & \text { LB } & \text { LB } & \text { LB } & \text { LB } & 0.6 & 1.8 & -1.4 & 2.9 & -5.1 & -5.1 & -4.6 \\ 3.0 \mathrm{~m} & \text { L LB } & \text { LB } & \text { LB } & \text { LB } & \text { LB } & \text { LB } & \text { LB } & \text { LB } & \text { LB } & \text { LB } & \text { LB } & \text { LB } & -2.0 & -8.0 & -1.5 & \text { LB } & \text { LB } & \text { LB } & -6.5\end{array}$

$L B=$ LAKE BOTTOM INTERFEFENCL

NM $=$ NOT MEASURED

POINT OF IRREGULAR VEPTICAL EXCESS-TEMPERATURE VARIATION: NONE.

FROM POINTS $7-25$, THE TEMPERATURE HALF-DEPTH OF THE STRATIFIED JET MAY BE ESTIMATED TO BE ABOUT 0.5-1.5 METERS.

POINTS OF IRREGULAR VERTICAL EXCESS-VELOCITY VARIATION: 18-25.

FROM POINTS 7-17, THE VELOCITY HALF-DEPTH OF THE STRATIFIED JET MAY BE ESTIMATED AS 0.5-1.5 METERS. 


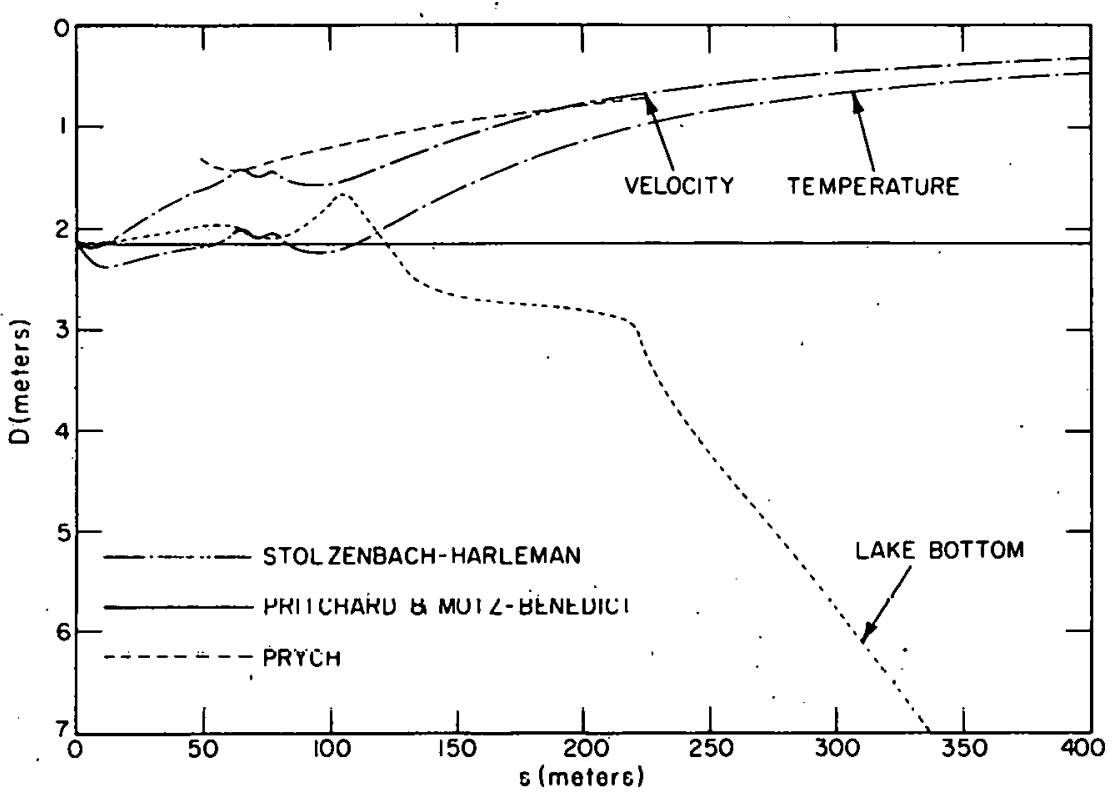

Fig. 77. Half-depths of Temperature and Velocity Distributions Resulting from Model Calculations for Palisades: October 10, 1972

three-dimensional Prych and Stolzenbach-Harleman models predict a buoyant surface jet, with only the Stolzenbach-Harleman temperature half-depth reaching the bottom. On a point-by-point basis (see Figs. 43-48), the temperatures at Stations 9, 11, 14, and 15 do not decline to half their near-surface values before the lake bottom is reached. All other points indicate a $1.0-$ to $1.5-\mathrm{m}$ temperature half-depth. It is inferred from the data that the jet hugs the bottom for the first $125 \mathrm{~m}$ and then rises to a 1.0 - to $1.5-\mathrm{m}$ half-depth as the bottom drops away and jet buoyancy becomes predominant. A point-by-point analysis of the velocity data indicates no real trend. The bottom was usually encountered on the first two transects before the half-depth was reached. 'The velocity half-depth appears to be 1.0-1.5 $\mathrm{m}$ otherwise. The variation of velocity data with depth at Palisades is just too. erratic for any consistent conclusions. This also was true for the Palisades data of June 14 and .Tuly 19.

\section{E. Isotherm Areas}

Isotherm areas were calculated based upon the temperature-distribution function resulting from the fit to the data by JETFIT. The data were not sufficiently detailed to contour isotherms and determine areas directly. Consequently, the JETFIT area calculations should be used with caution. It is encouraging, however, to note that isotherm areas for the four dates at Point Beach are approximately the same; this is to be expected, since plant conditions were nearly the same and ambient currents were weak, if present at all. These isotherm areas, along with the model predictions, are presented in Figs. $78-82$. 


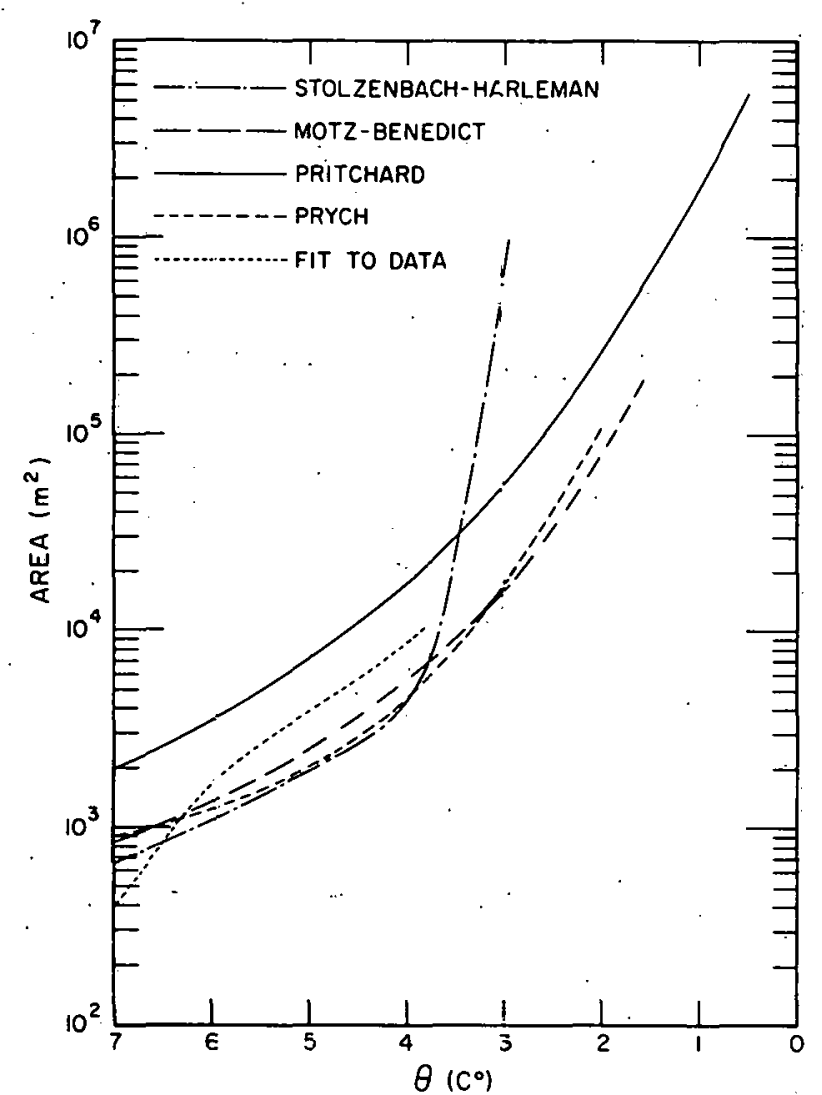

Fig. 78. Isotherm Areas Resuliting from the Fitting Procedure and N-odel Calculations for Point Beach: May 18, 1972. ANL Meg. No. 190-939.

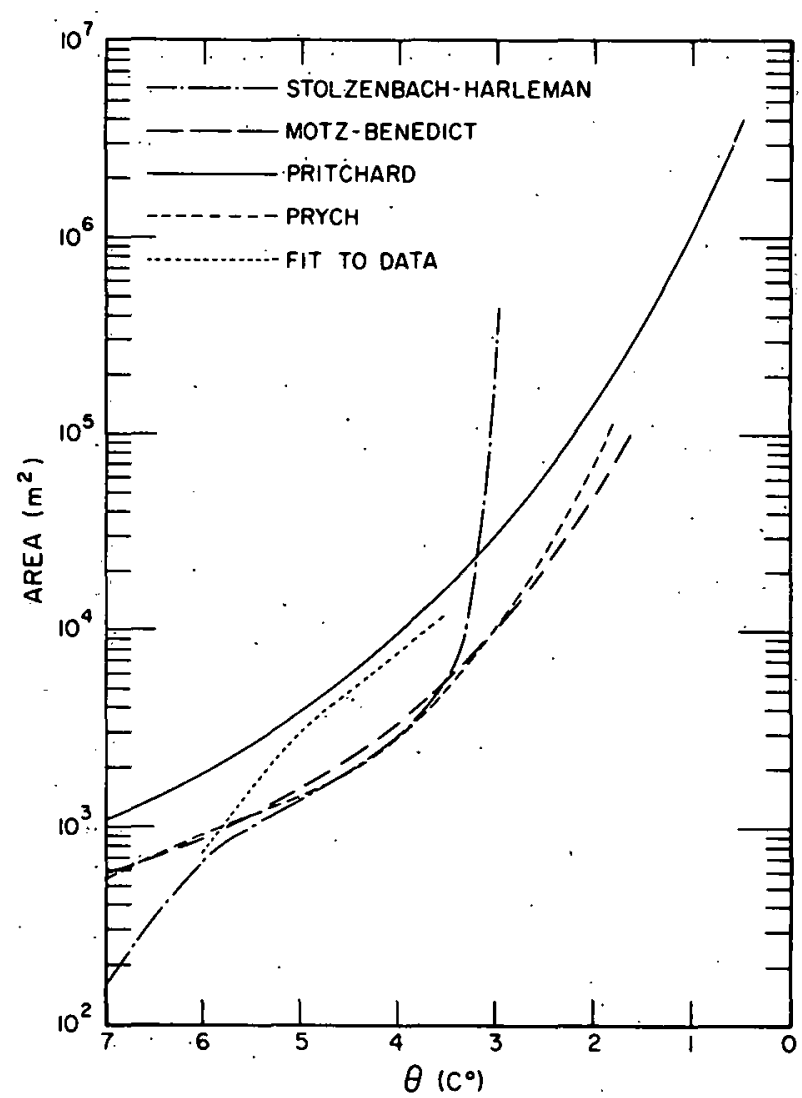

Fig. 79. Isotherm Areas Resulting from the Fitting Procedure and Model Calculations for Point Beach: May 23, 1972. ANL Neg. No. 190-938: 


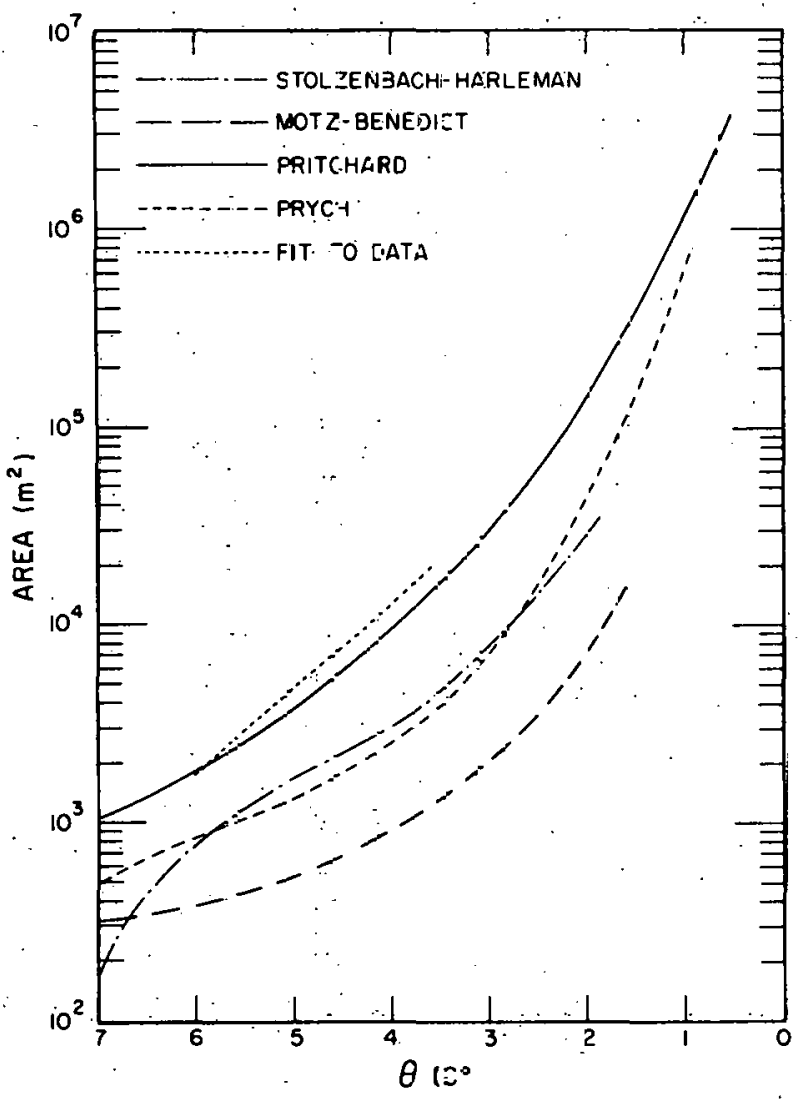

Fig. 80. Isotherm Areas Restlting from the Fitting Procedure ajd Model Calculations for Point Bezch: July 13, 1972. ANL Neg. No. 191)-943.

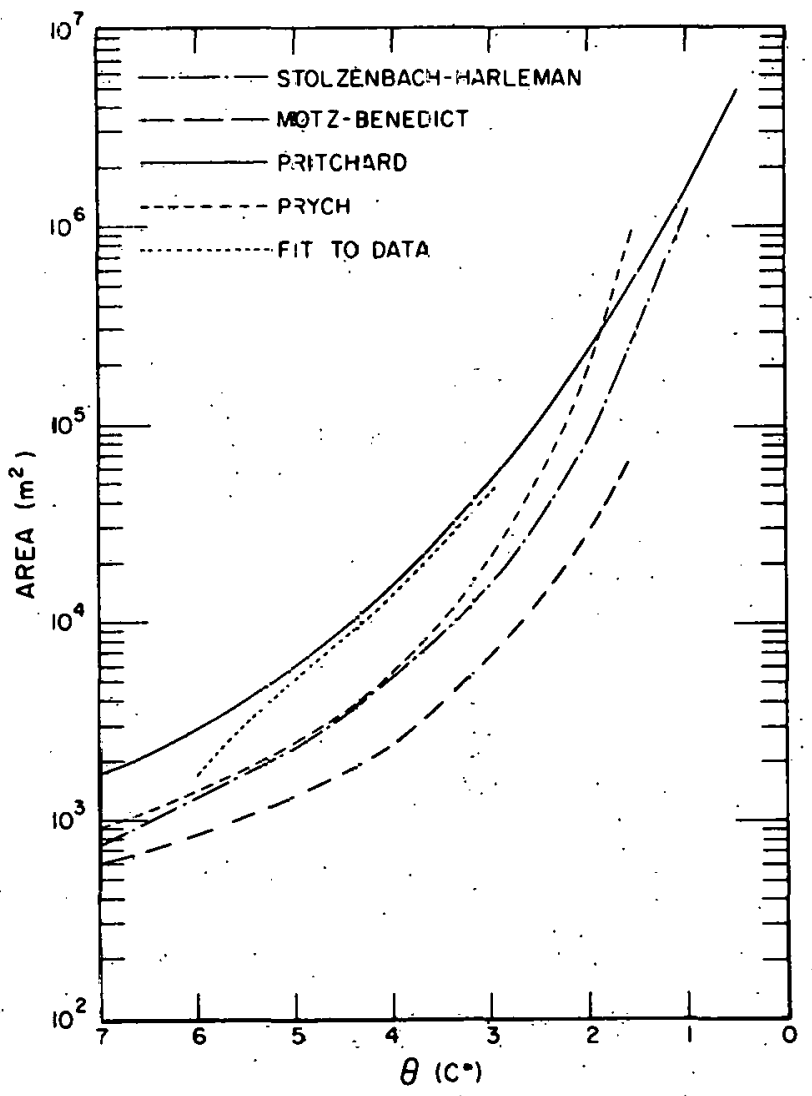

Fig. 81. Isotherm Areas Resulting from the Fitting Procedure and Model Calculations for ?oint Beach: September 9, 1972. ANL Neg. No. 196-955. 


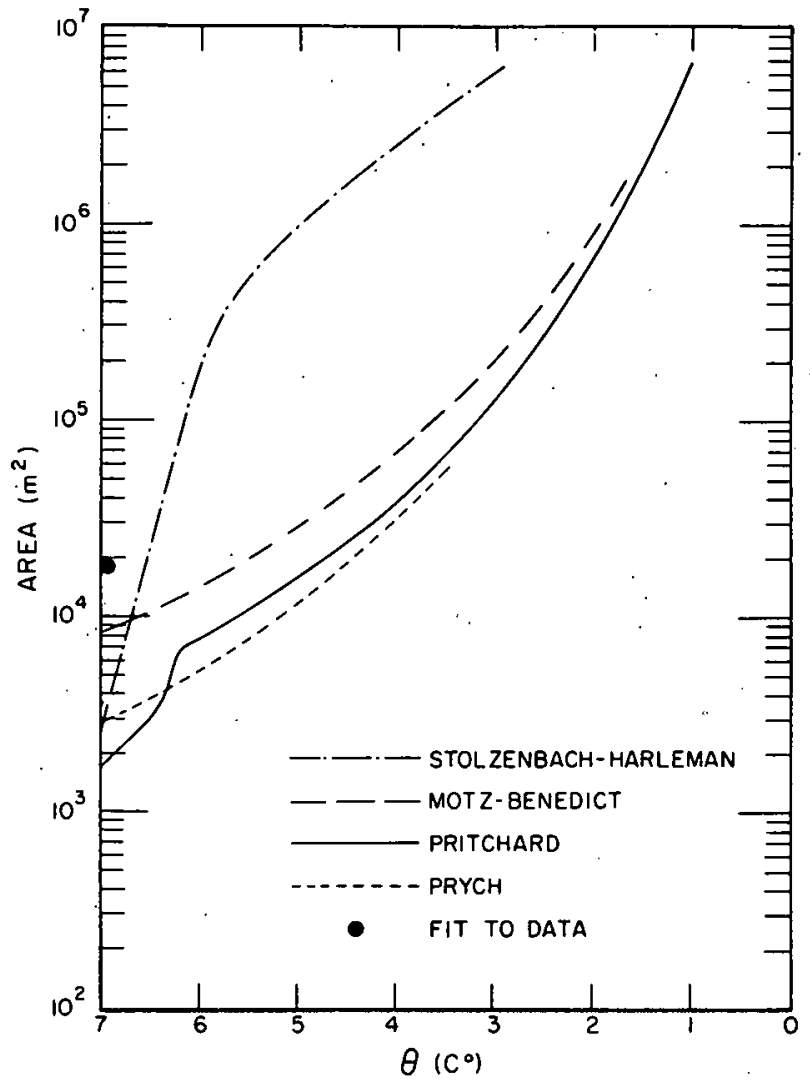

Fig. 82. Isotherm Areas Resulting from the Fitting Procedure and Model Calculations for Palisades: October 10, 1972. ANL Neg. No. 190-937.
The Pritchard model appears to be most accurate in area predictions for the four Point Beach dates. Any discrepancies appear to be on the conservative side. For the nocurrent cases (Figs. 78, 79, and 82), the areas predicted by Stolzenbach and Harleman are reasonable but optimistic for the region in which data were taken (to $3-4 \mathrm{C}^{\circ}$ i sotherm). Beyond the data, however, the model predicts sharply increasing areas, perhaps due to the large lateral spreading predicted. The current cases of July 13 and September 9 reveal a less steep increase in areas with decreasing temperature excess; the model for those dates tends to underpredict areas for the region in which data exist. Too sharp a distinction between the no-current and weak-current cases appears in the area predictions. The StolzenbachHarleman model as derived is too complex to allow any speculation as to possible reasons for that behavior.

The Prych model gives reasonable areas, but they are consistently lower than the data. Also, the predictions are nearly the same for all dates. This is to be expected, since the plant and environmental conditions varied only slightly from date to date at Point Beach. Motz-Benedict predictions are consistently lower on all dates. A different choice of parameters in this regard could affect these results greatly.

The Palisades area calculations are difficult to evaluate. Most of the jet data taken appears to be in the region of flow establishment. Although the actual excess temperature was $9.2 \mathrm{C}^{\circ}$, the data fit indicated a temperature excess of $7.8 \mathrm{C}^{\circ}$ for a good $200 \mathrm{~m}$ offshore. The models all underpredict areas at the $7 \mathrm{C}^{\circ}$ isotherm shown by the dot on Fig. 82 ; little can be said beyond that isotherm, since no data exist. The Pritchard curve has a sharp increase in area at approximately the $6 \mathrm{C}^{\circ}$ isotherm due to Pritchard's method of halting vertical entrainment when a predicted plume depth of $3.05 \mathrm{~m}$ (10 ft) is reached.

F. Decay of Centerline Temperature and Velocity with Depth

Observation of the Point Beach field measurements with depth reveals an approximate constancy of temperature and velocity for the depths measured 
(to a depth of 2.5-3.0 m). It is expected that, beyond the 2.5-3.0 m of uniform profile, there exists a vertical region for which temperature and velocity ex-
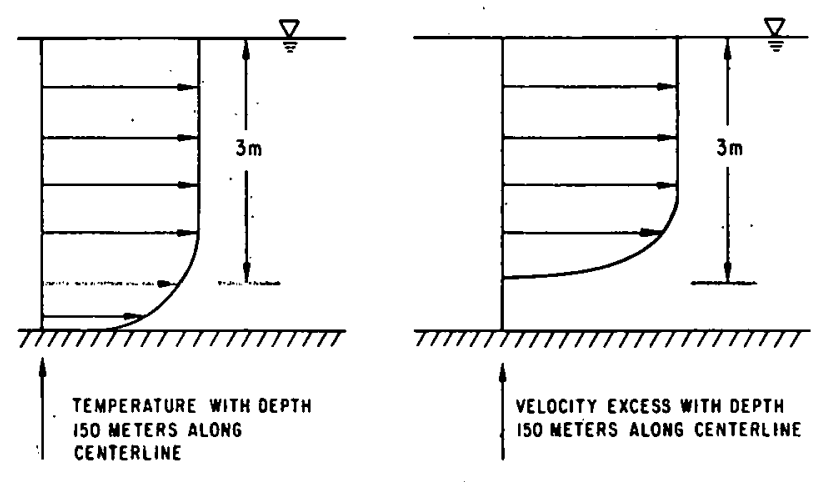

Fig. 83. Idealized Decay of Temperature Excess and Velocity Excess with Depth cesses decrease to zero (see Fig. 83). These vertical distributions are perhaps reminiscent of fully developed turbulent flow in pipes, which consists of a large uniform temperature and velocity core, combined with a sharp approach to the boundary value (wall of pipe, or here, the lake bottom).

The results of the data-fitting procedure for Point Beach appear in Figs. 84-87. The points in these four figures are the centerline temperature and velocity excesses from

the titting procedure at $150 \mathrm{~m}$ from the outfall along the centerline. Model predictions of temperatures and velocities with depth at $150 \mathrm{~m}$ are also plotted. The lake depth at this distance is about $3.0-3.5 \mathrm{~m}$.

The chosen distance of $150 \mathrm{~m}$ is about two to three times the length of the region of flow establishment; only the two-dimensional models predict uniformity of vertical profiles at that distance. Although predictions of the Prych and Stolzenbach-Harleman models look poor, their predictions for the centerline values are too low, biasing the entire vertical-distribution predictions. More accurate centerline predictions would perhaps have yielded better agreement with the data. The three-dimensional models evaluated here predict too rapid a temperature and velocity decay with depth. The flowestablishment assumptions for vertical temperature decay of Stolzenbach and Harleman (constant-temperature vertical core, with small, turbulent boundary layer below it) also appear to be appropriate in the region of established flow. The Stolzenbach-Harleman decay with depth beyond the region of flow establishment is essentially parabolic, with no jet effects beyond a certain depth. The Prych decay is Gaussian in the vertical direction. The Pritchard and Motz-Benedict models, being two-dimensional, predict no change in behavior with depth.

The Palisades data are more of an enigma (see Fig. 88). The centerline distance of $150 \mathrm{~m}$ is expected to border the end of the region of flow establishment, yet the vertical temperature and velocity decay appears quite sharp. The depth of constant temperature, if any, is quite small. Complicating factors here are the irregular shallow bottom, approximately $2 \mathrm{~m}$ in depth, a diverging surface outfall structure, and a nonuniform velocity distribution. More detailed data would be required before further conclusions could be drawn. 


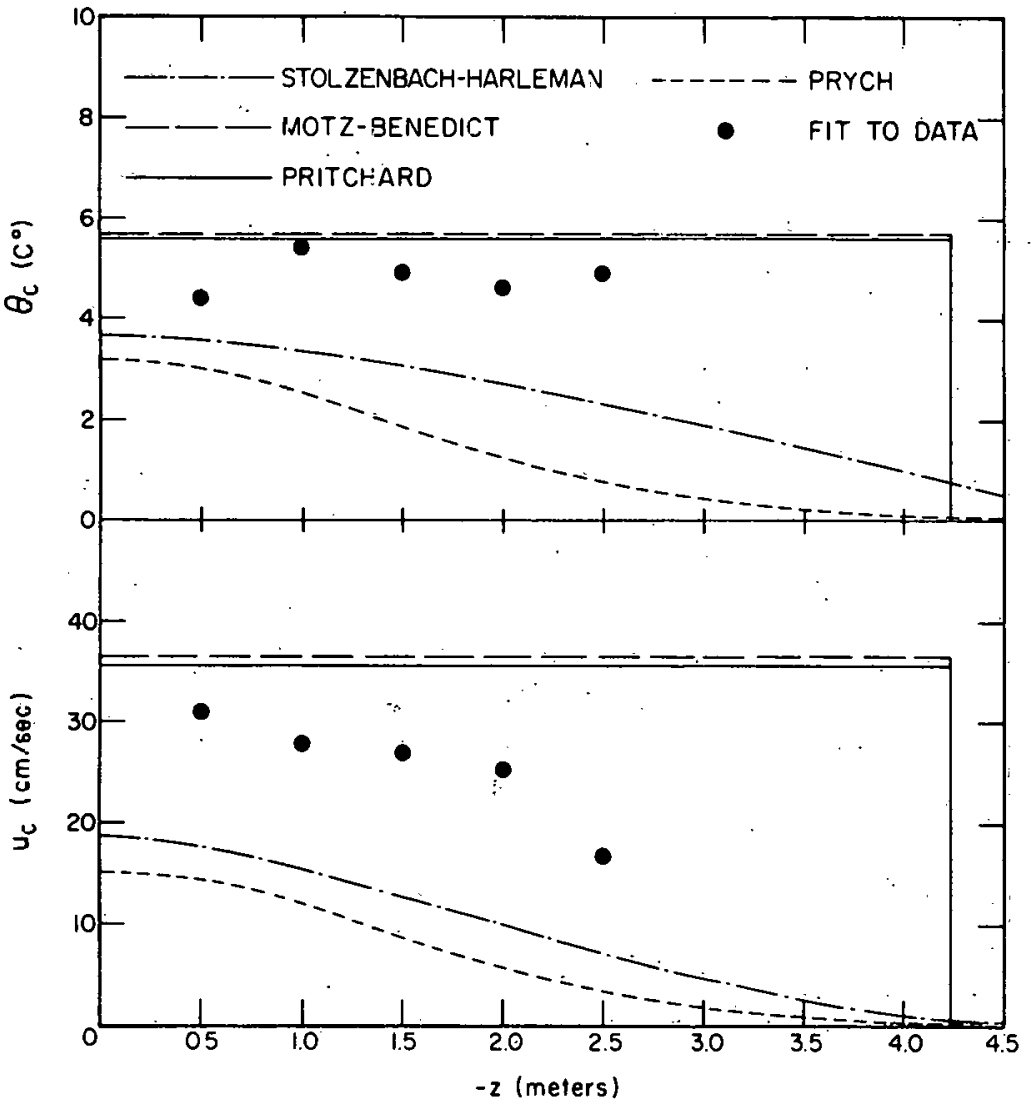

Fig. 84. Centerline Temperature Exçess and Vèlocity Excess as a Function of Depth at $150 \mathrm{~m}$ from Outfall Resulting from the Fitting Procedure and Môdel Calculations for Point Beach: May 18, 1972

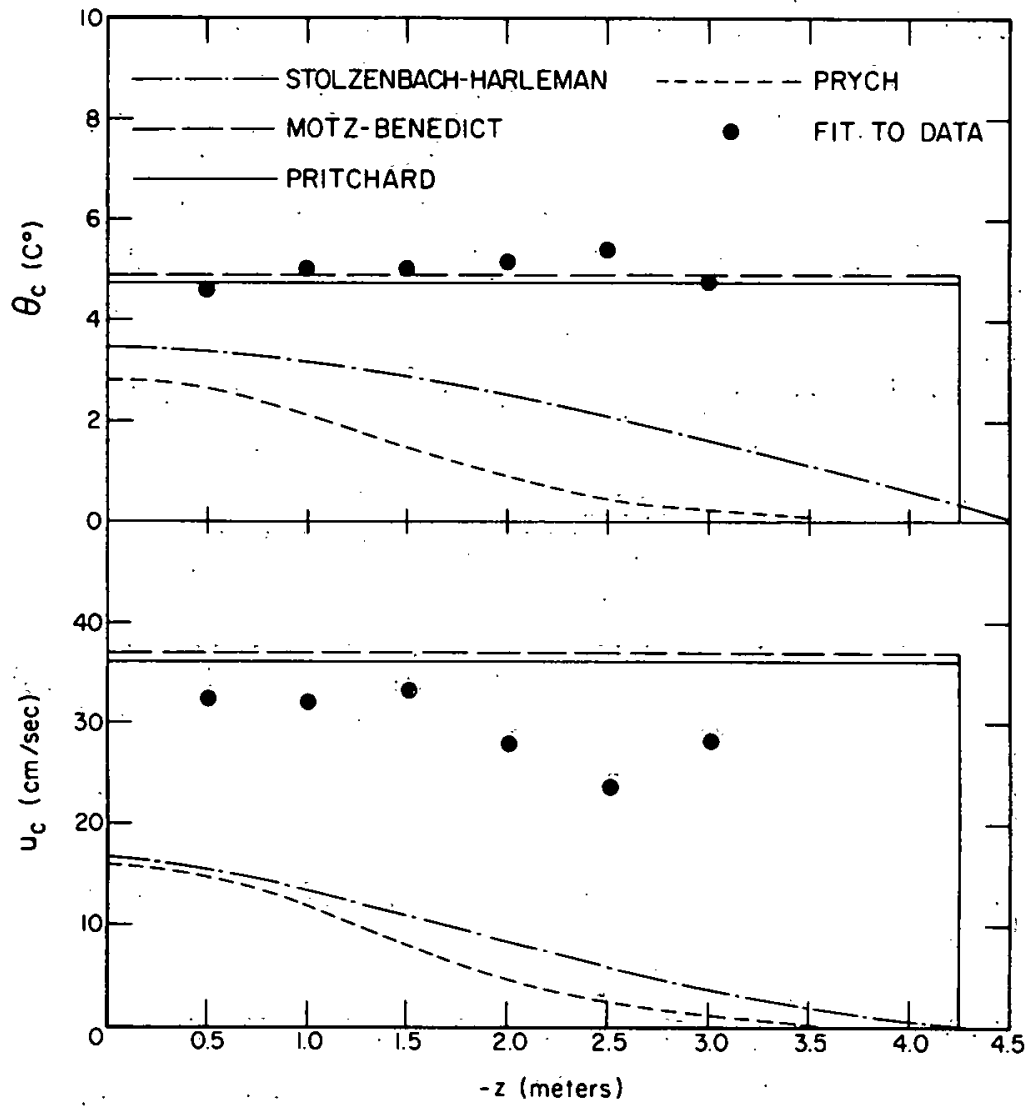

Fig. 85. Centerline Temperature Excess and Velocity Exces̀s as a Function of Depth at $150 \mathrm{~m}$ from Outfali Resulting from the Fitting Procedure and Model Calculations for Point Beach: May 23, 1972 


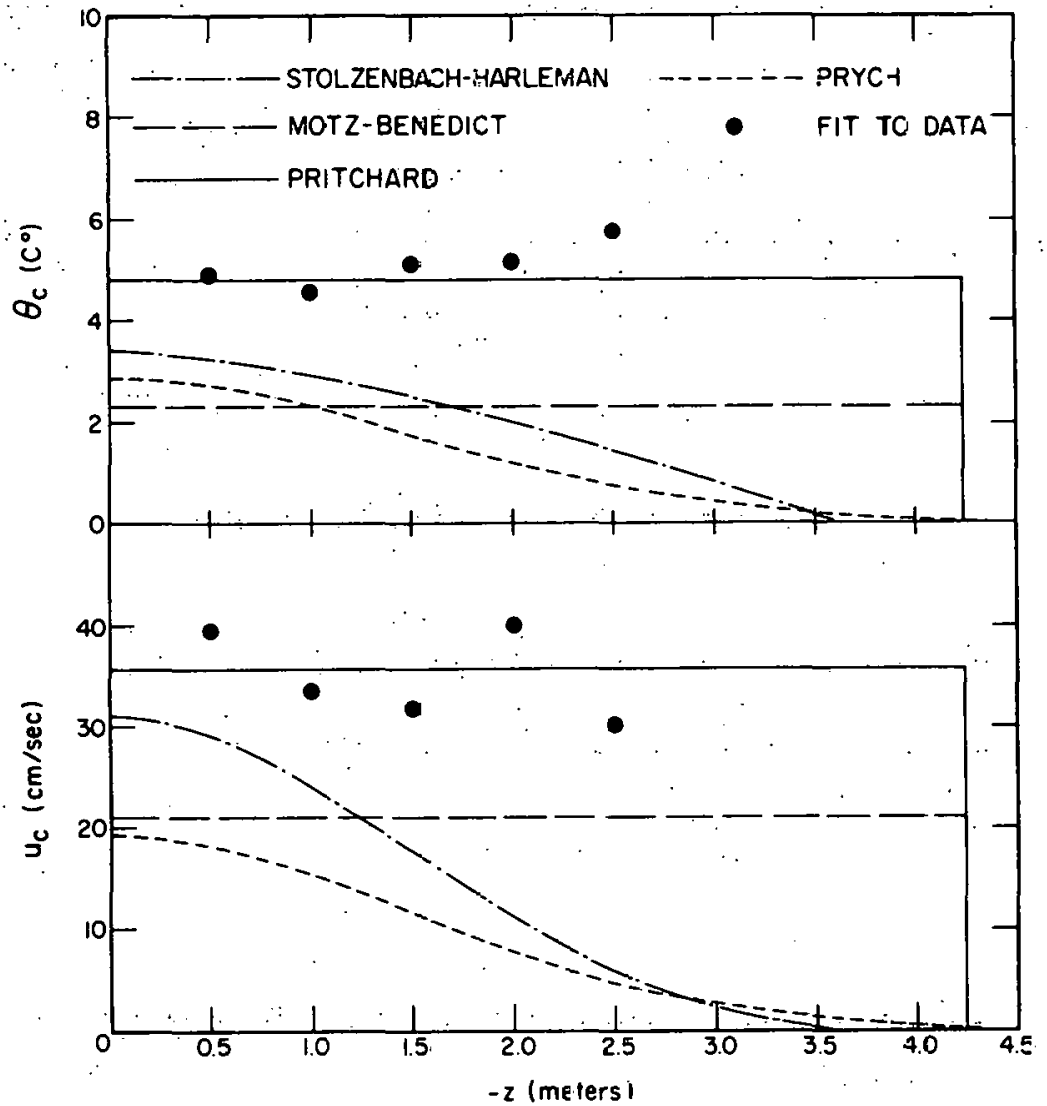

Fig. 86. Centerline Temperature Excess and Velocity Excess as a Function of Deptin at $150 \mathrm{~m}$ from Outfall Restiliting from the Fitting Procedure ard Model Calculations for Point Beach: July 13, 1972

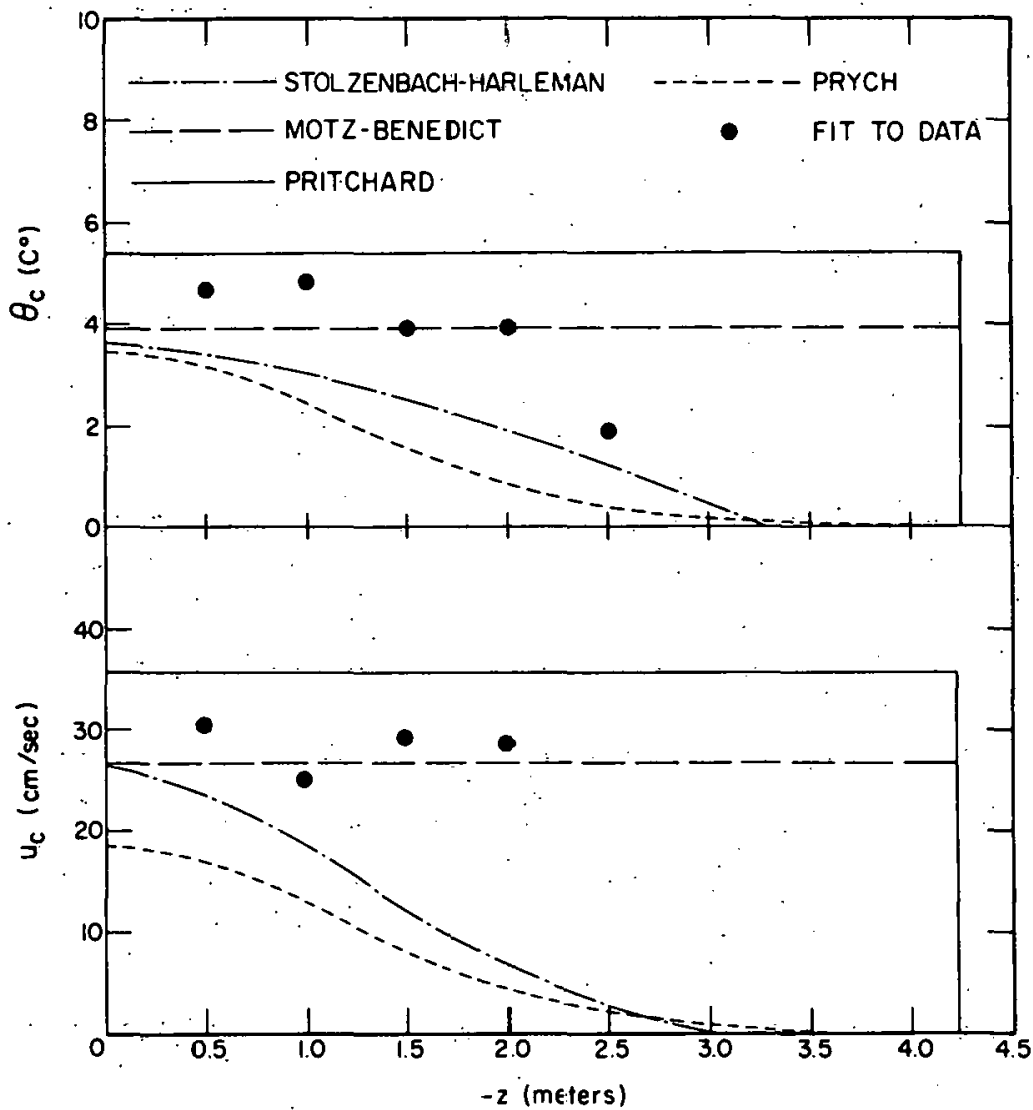

Fig. 87. Centerline Temperattre Excess and Velocity Excess as a. Function of Depth at $150 \mathrm{~m}$ from Outfall Resulting from the Fitting Procedure and. Model Calculations for Point Beach: Seprember 9, 1972 


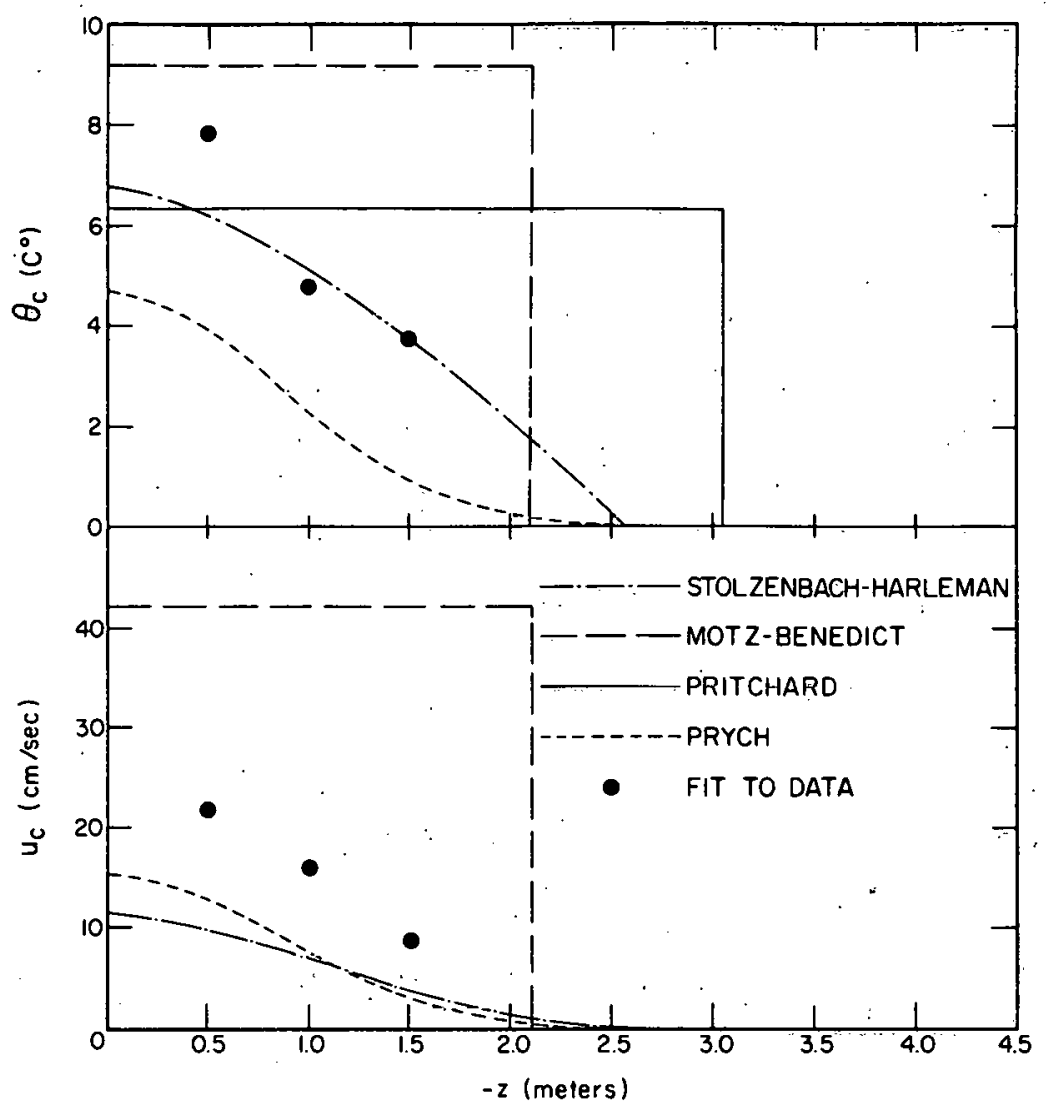

Fig. 88. Centerline Temperature Excess and Velocity Excess as á Function of Depth at $150 \mathrm{~m}$ from Outfall Resulting from the Fitting Procedure and Model Calculations for Palisades: October 10, 1972

G. Variation of Temperature and Velocity Width with Depth

Each mathematical model predicts a constant velocity and temperature width with depth. The two-dimensional models do so by the nature of their twodimensionality, the three-dimensional models by definition of their lateral and vertical profiles. The Stolzenbach-Harleman and Prych models assume, for any cross section of the jet normal to the centerline, the same decay laterally independent of depth, as well as identical decay vertically, irrespective of . lateral distance. Consequently, the widths must maintain a constant value with depth.

The data, however, show a more lens-shaped profile, indicating that the temperature and velocity widths decrease with depth (see Figs. 89-92). The local centerlines (with depth) do not coincide, yet are sufficiently close to indicate that such vertical profile results may be meaningful. From the figures, the temperature width appears to be uniform with depth for about $1.5 \mathrm{~m}$ and then decreases abruptly; the velocity width appears constant for a shorter depth distance $(0.5-1 \mathrm{~m})$ before decreasing toward ziero. 


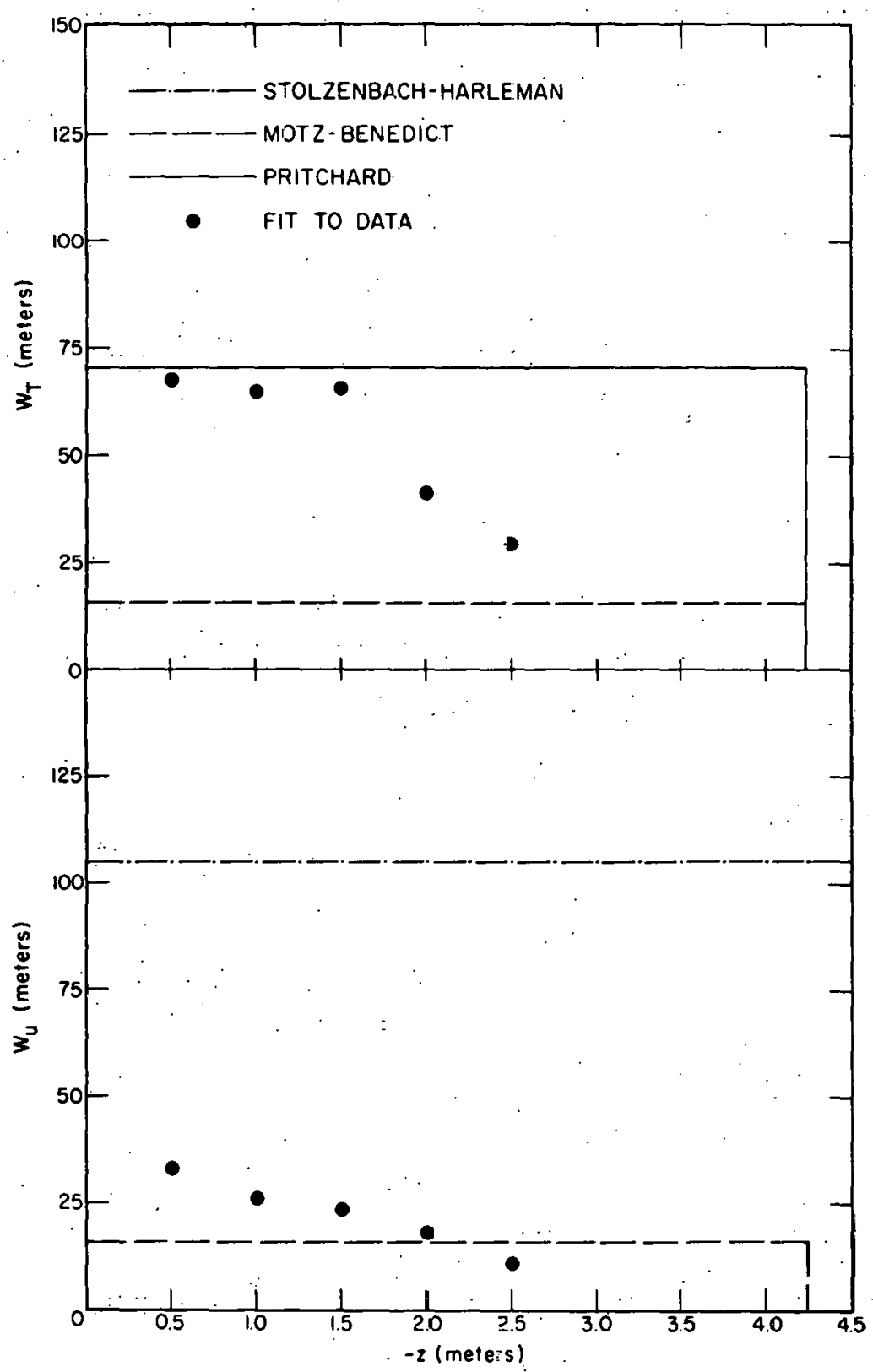

Fig. 89. Half-widths of Temperature and Velocity Distributions as a Function of nepth a: $150 \mathrm{~m}$ from Outfall Resultirg from the Fitting Procedure Model Calculations for Foint Eeach: May 18, 1972

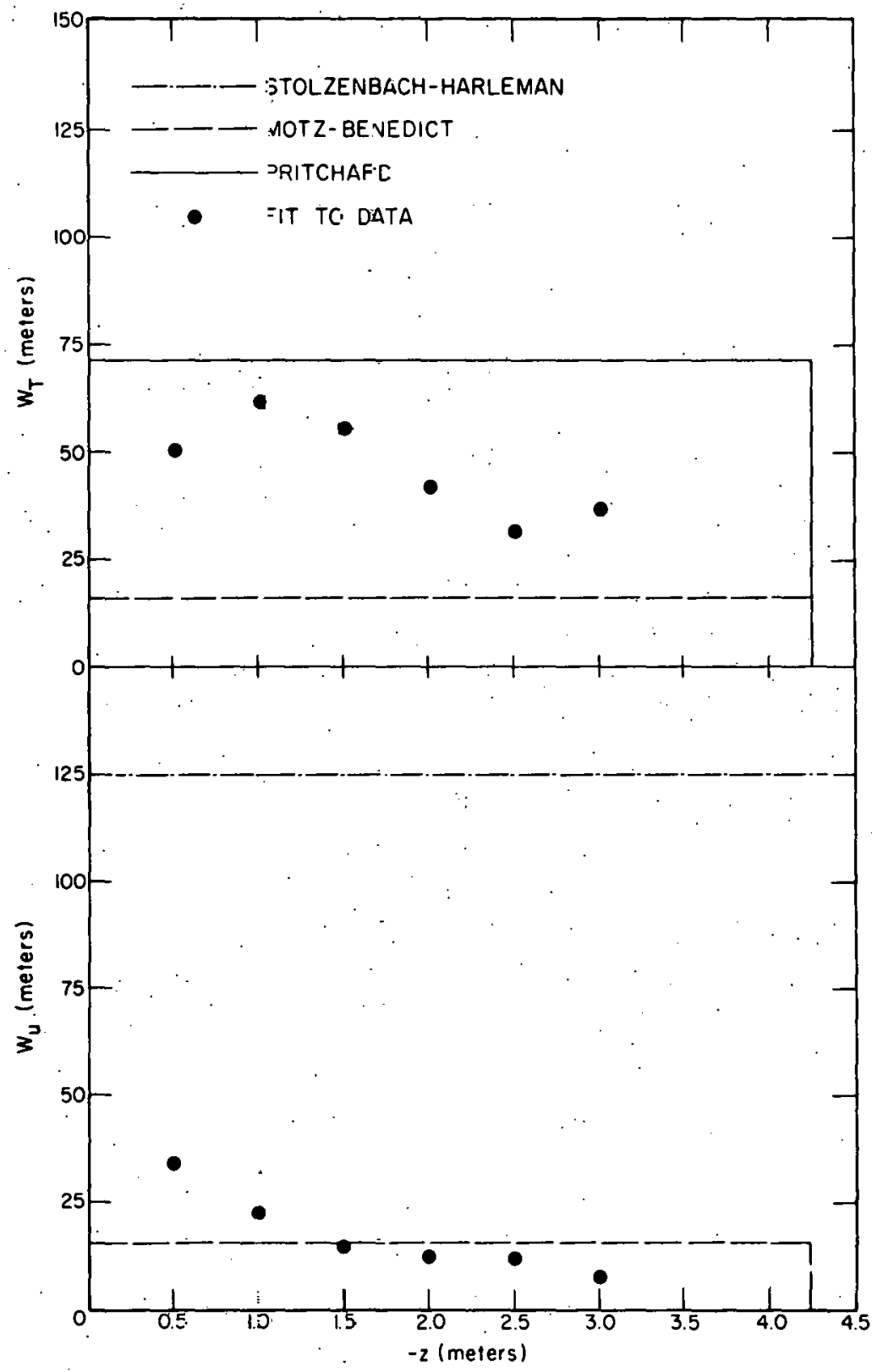

Fig. 90. Half-widths of Temferature and Velocity Distributions as a Function of Depth at $150 \mathrm{~m}$ from Outfall Resulting from the Fitting Procedure and Model Calculation: :or Point Beach: May 23, 1972 


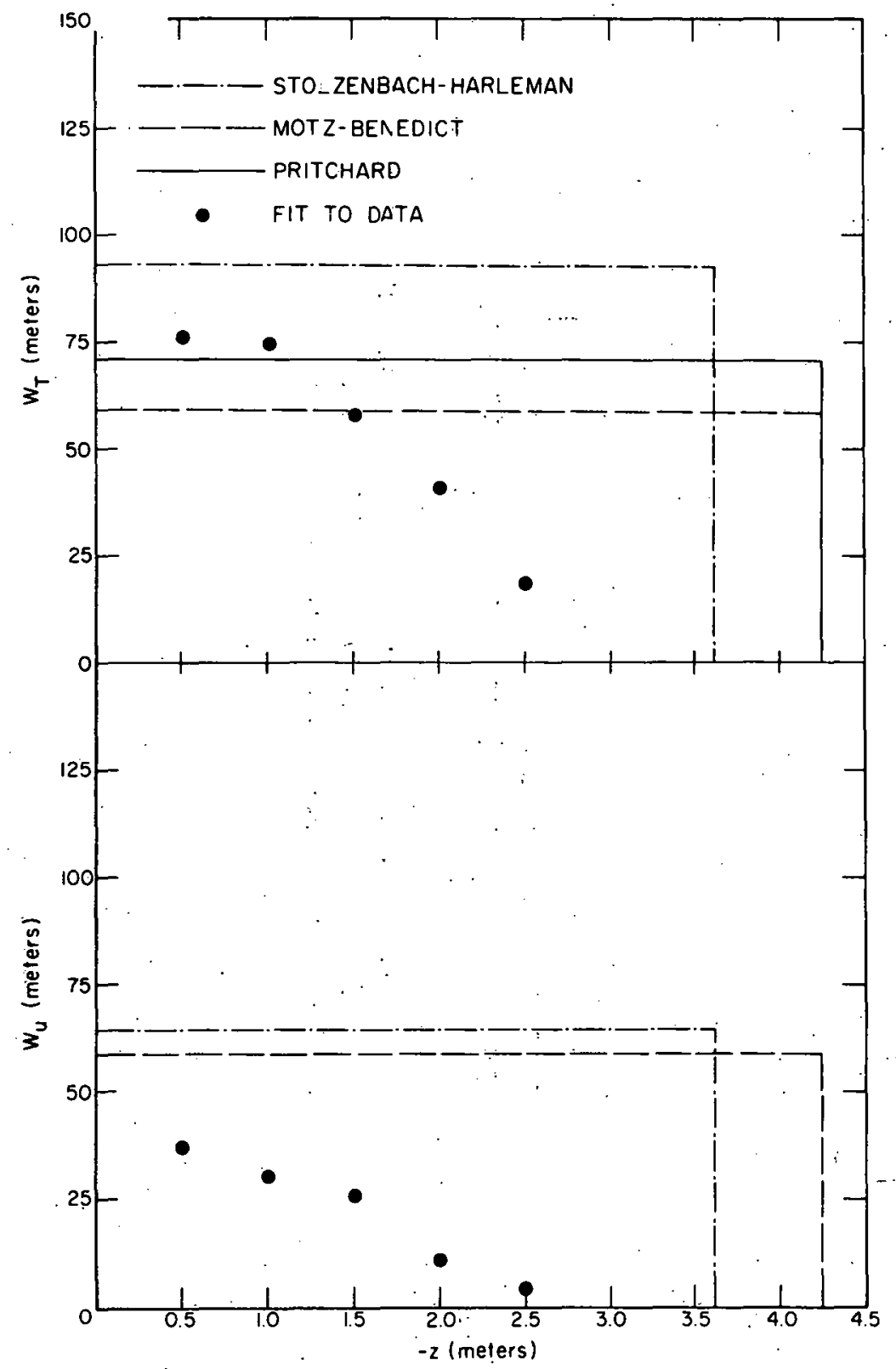

Fig. 91. Half-widths of Temperalure and Velocity Distributions as a Function of Depth at $150 \mathrm{~m}$ from Outfall Resulting from the Fitting Procedure and Model Calculations for Point Beach: July 13, 1972

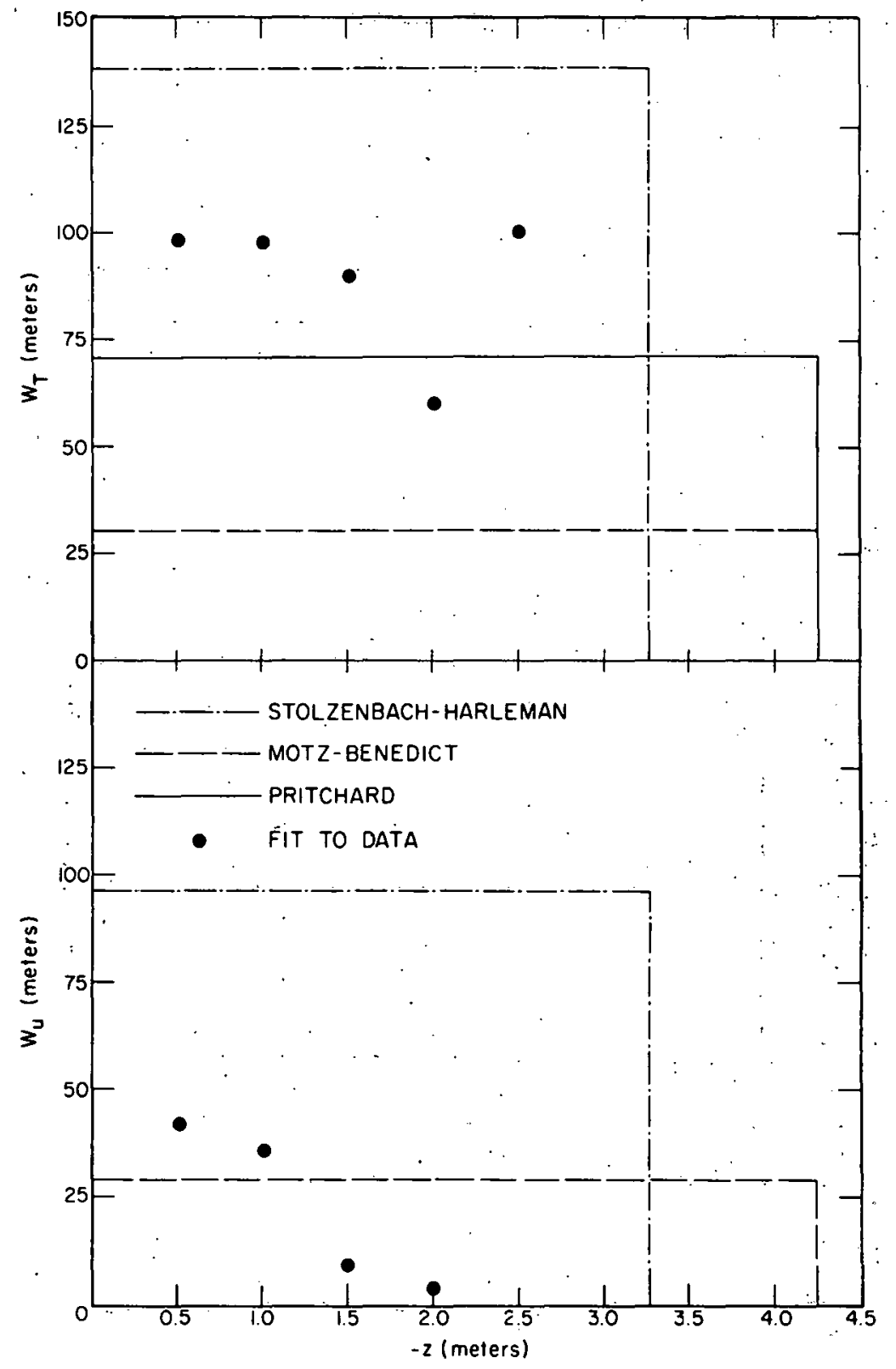

Fig. 92. Half-widths of Temperature and Velocity Distributions as a Function of Depth at $150 \mathrm{~m}$ from Outfall Resulting from the Fitting Procedure and Model Calculations for Point Beach: September 9, 1972 
The Palisades data (see Fig. 93) are insufficient and irregular, due again perhaps to the shallow lake depths in the discharge vicinity as well as the nonuniformity of the diverging discharge.

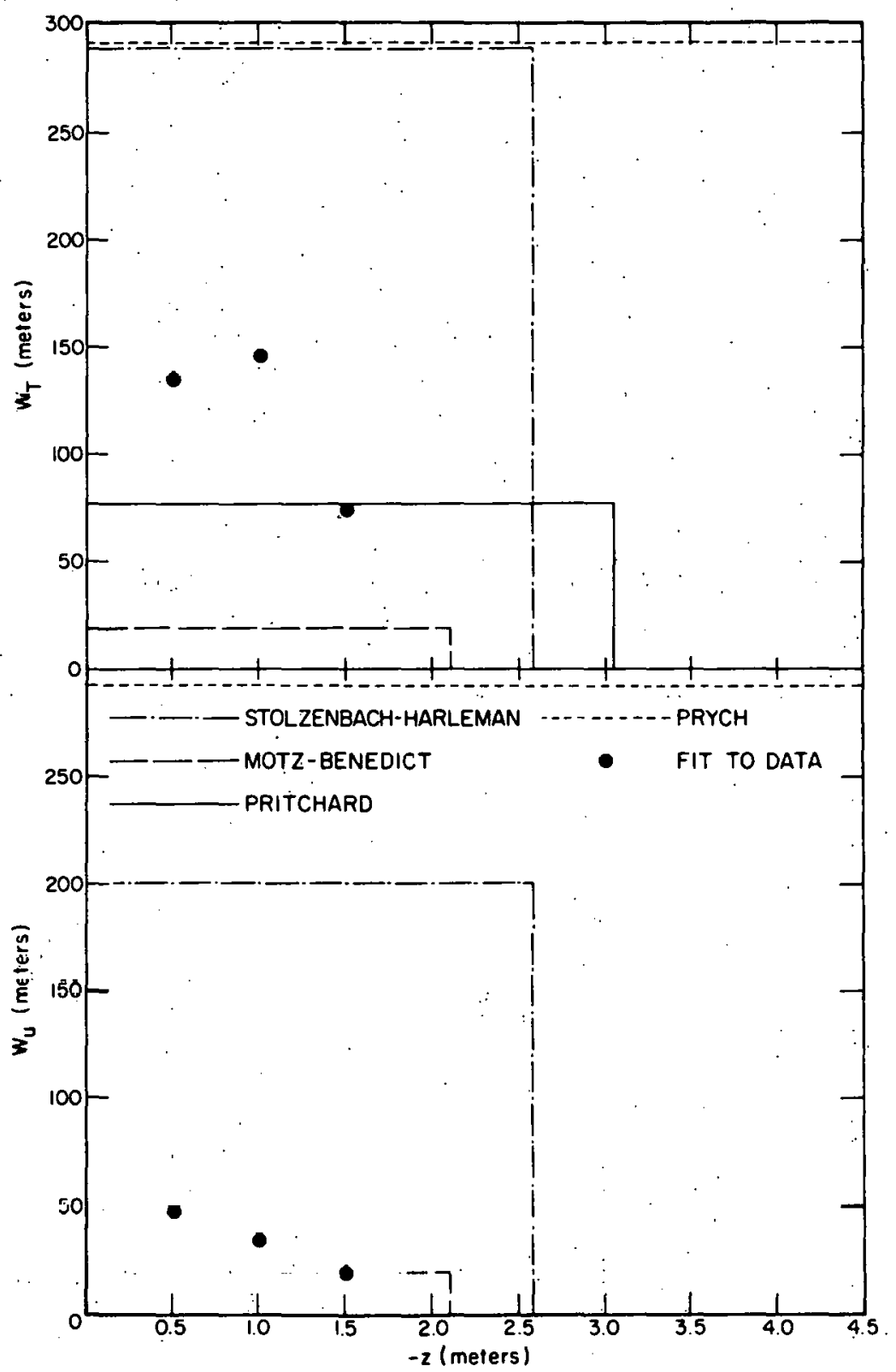

Fig. 93. Half-widths of Temperature and Velocity Distributions as a Function of Depth at $150 \mathrm{~m}$ from Outfall Resulting from the Fitting Procedure and Model Calculations for Palisades: October 10, 1972 


\section{SUMMARY AND CONCLUSIONS}

A. Field-data Acquisition

The experimental technique described in Sec. II for making measurements in the jet regime represents a compromise between speed and completeness. More data in a shorter period of time are needed for a more accurate comparison with model predictions. Given the manpower and equipment limitations under which the work was done, the technique reported on in this report gives reasonable results in terms of number of data points versus time of measurement. The data obtained in this manner reflect the gross features of the jet regime, but have two limitations:

1. Since the temperature and velocity at a point in the jet are not constant, a single measured value of each may not accurately define these parameters at a point.

2. The measurements are not made simultaneously. Typically, they are collected over a period of 3-7 hr during which time factors affecting the jet may change.

B. Data-smoothing Technique

The fitting procedure described in Sec. V was used to extract the gross features of the temperature and velocity distributions from the field measurements. Some procedure of this type is necessary because the data consist of measurements at isolated points in the temperature and velocity fields. The functional forms used in this study were chosen for several reasons. One reason is their similarity to the forms resulting from the simple yet fairly widely used jet analyses of Pritchard ${ }^{7}$ and Motz and Benedict. ${ }^{5}$ Another reason is that preliminary examination of the Point Beach data indicated that such forms might represent the available data fairly well. Finally, it was thought that for this initial attempt at data fitting, simple forms with limited numbers of free parameters would be appropriate. Clearly, unless the actual functional forms are known, any fitting procedure of this type will have limitations and biases.

The results of the present fitting procedure can be considered to be a successful first attempt. It is difficult to assess the goodness of fit in a meaningful, quantitative way; the refore a point-by-point tabulation has been included in Appendix D. The average deviations of the fitted function from the data for any particular set of measurements never exceeded $1.1 \mathrm{C}^{\circ}$ for temperature and $8 \mathrm{~cm} / \mathrm{sec}$ for velocity. These values correspond to 12 and $14 \%$ of the initial excess temperature and average outfall velocity, respectively. Certainly at least the gross behavior of the near-field temperature and velocity distributions has been accounted for with the present fitting procedure. 


\section{Analytical Model; Field-data Comparisons}

The results of the model-data comparisons are summarized here for each model evaluated.

\section{Pritchard Mode1}

The Pritchard model compared best with the Point Beach Unit 1 and Palisades jet data presented in this report. Model-data discrepancies usually indicated conservative predictions on the part of the model. The success of Pritchard's model for these cases is due in part to the persistent efforts of the author in calibrating the model with field and hydraulic. dat.a. Also, the field data used by Pritchard in the development of his model were generally from plants with similar discharge and environmental rharacteristics to Point Beach and Palisades.

This basically empirical or phenomenological model is not expected to perform well when buoyancy is significant (initial densimetric Froude number less than about 2) or when the dilution capacity of the receiving water is significantly restricted by lateral or bottom boundaries. The model is also weak in its treatment of vertical mixing; the model has scarcely been tested for prototype situations in which vertical entrainment is significant.

Although the model is too simplistic to handle the major complications that may be important in certain field cases (buoyancy, cross currents, etc.), it might well give good results for cases of the higher-velocity discharges (i.e., larger densimetric Froude number problems) for the critical case of a stagnant lake. Further verification work for this model is desirable at different sites describing a variety of geometric, kinematic, and dynamic conditions.

\section{Motz-Benedict Model}

The Motz-Benedict recommendations for the choice of entrainment coefficient $\mathrm{E}$ apparently vary over too large a range for the data observed at Point Beach and Palisades. The recommended value of 0.05 for the stagnant-lake case gave a reasonably good approximation for centerline temperature and velocity decay. Values of $E$ for $A$ (ratio of ambient velocity to initial jet velocity) between 0 and 0.2 were determined by a linear interpolation from the known values of $0.05(A=0)$ and $0.4(A=0.2)$. This appeared to $u$ us to be as good an approximation for $E$ as any in the presence of the wide scatte $r$ in the Motz-Benedict data from which $E$ is to be determined. The results indicate that smaller values of $E$ for the cases of nonzero ambient crosscurrents would have been more appropriate. Temperature and velocity decays would not have been as rapid and plume widths would have been larger.

The most sensitive parameters are $E$ and $r=b_{0} / b_{0}^{\prime}$. Data for the determination 
of $E$ have much scatter; data for $r$ have not been determined. The inadequacies of this simple, two-dimensional model are briefly summarized as follows:

a. Buoyant forces, as well as vertical entrainment, are ignored in the model development. For densimetric Froude numbers less than about 2, buoyant spreading is quite important; for densimetric Froude numbers exceeding about 5, a substantial amount of vertical entrainment may be expected. Consequently, the types of discharges for which the model may be used is limited. The entrainment coefficient, determined from data fitting, must actually account for spreading due to buoyancy as well as from jet entrainment.

b. The choice of a coefficient of entrainment must be based on inconsistent data as expressed above. Data presented by Motz and Benedict show considerable variation in the value of entrainment coefficient from situation to situation. Particularly for lake data, the value of $E$ is shown to be extremely small, $\sim 0.04$, in relation to riverine situations where $E$ is about an order of magnitude larger. A particularly interesting contrast is afforded when one compares ${ }^{5}$ the Motz-Benedict laboratory data for a $90^{\circ}$ discharge to the Romberg-Ayres lake data. Although most of the initial conditions are similar, the entrainment coefficients a re different by about a factor of 10 . Part of the problem probably lies with the fact that Motz and Benedict analyzed lake data from locations in which prominences (breakwaters, for example) were present. A second difficulty is that the limited field results indicated that the entrainment coefficient remained essentially constant at a particular location with changing values of $A$; this behavior is consistent with the laboratory findings showing $E$ to be relatively independent of $A$ for each site. Here, turbulent intensity of the ambient current (see item e below) may be a significant factor that was ignored.

c. We believe the model is actually restricted to small ambient currents. The simulation assumes that the jet velocity approaches zero at large distances normal to the jet centerline: The assumption of Gaussian profiles for temperature and velocity and the assumption of equal rates of entrainment on offshore and lee sides of the jet are not valid for ambient currents that are not very small.

d. The model does not simulate unequal rates of spread for momentum and heat, as has been observed to be significant in the Point Beach and Palisades data. The Prych and Pritchard models also do not distinguish between these rates of spread.

e. The model does not treat turbulent intensity in the crossflow, due to the assumption that such turbulence and its effect on mixing are negligible. Although some account of this effect may be made in the choice of entrainment coefficient, any such treatment would be only qualitative in nature. The other models do not treat this phenomenon adequately either; the Prych model does include ambient turbulence in terms of a horizontal and vertical ambicnt cddy-thermal diffusivity. 
Deficiencies $a$ and $b$ could eventually be fatal to the model. Improved predictions might be obtained by determining the free parameters

$$
\begin{aligned}
& E=\text { entrainment coefficient, } \\
& r=b_{0} / b_{0}^{\prime}
\end{aligned}
$$

and

$\mathbf{s}=$ ratio of lateral spread of heat to that of momentum

for each set of data available, with the hope that a consistent trend (or correlation) might develop as the initial densimetric Froude number, initial angle of jet discharge with the current; velocity ratio $A$, and $w$ (ambient stream width/discharge width) vary.

\section{Stolzenbach-Harleman Mode1}

The Stolzenbach-Harleman model generally compared poorly with the jet data of Point Beach and Palisades. Centerline temperature and velocity decay were predicted to be too rapid; the lateral spread of the jet was much too great. The model does not consider jet interaction with the lake bottom, which does occur to some degree near the outfall. Such interaction should provide some increase in lateral spread due to restricted dilution at the jetlake bottom interface. The predicted lateral spread, however, greatly exceeded the observed spread, even with bottom effects assisting that lateral growth. Surprisingly, the model tends to underpredict lateral spread in the region of flow establishment. These poor comparisons of model to data may be traced, in part, to the model assumption on lateral-spreading velocity, which was based more on physical intuition than on any data.

A second major fault of the model lies in the presumed jet structure based upon nonbuoyant jet theory. First, the four-zone, rectangular jet stîcture may not be valid for buoyant jets. In particular, the cross section of a buoyant jet is normally taken to be lens-shaped rather than rectangular, as supposed by the model. The assumed division of the jet into four distinct regions necessitates that interregional velocities be specified. The forms nf these velocities are unknown and therefore must be guessed. Stolzenbach and Harleman also require that no turbulent momentum transfer occurs between regions of the jet or between the jet and ambient water. This is tantamount to dropping the Reynolds stress terms in the equations of motion or, equivalently; dropping the turbulent-diffusion mechanism. As a consequence, turbulent jet diffusion had to be artificially simulated through the entrainment coefficient and similarity forms for temperature and velocity. In any case, some calibration of the model to actual field and hydraulic data might have provided better predictions.

Aside from the largely theoretical criticisms of the model, the re a re practical difficulties in actually obtaining a nume rical solution to the set 
of ordinary differential equations. Due to the complexity of the set, derivatives must be found by solving a linear set of algebraic equations before applying a Runge-Kutta scheme. For many cases, the matrix, which must be reduced, is nearly singular and much precision is lost in solving for the derivatives. Among the problems that may be encountered in using the code are:

a. Width predictions may decrease by as much as $50 \%$ with each order-of-magnitude reduction in the errox criteria until the program finally fails!

b. The program may not run for cases of

(1) Low initial densimetric Froude number.

(2) Low aspect ratio.

(3) Initial angles greater than $90^{\circ}$.

c. Nume rical underflows must be suppressed for successful completion of many runs.

d. Differences in machine precision due either to differences in word structure or to differences in the operating system may cause differences of up to $5 \%$.

\section{Prych Model}

The Prych predictions have the same problems as those of Stolzenbach and Harleman:

a. Too rapid a decay in centerline temperature and velocity.

b. Too great a lateral spread.

The Prych model also compares poorly with the Point Beach and Palisades data.

We suspect that a major difficulty with the theoretical development is in the assumption for a lateral-spreading velocity based upon the analogy to the celerity of a density front of a uniform depth with a uniform density difference. This model for lateral spreading is apparently incorrect as simulated. Also, the hydrostatic pressure force is simulated to act in the longitudinal direction only. Pressure forces in reality act longitudinally and laterally, with an approximate hydrostatic distribution vertically. The as sumption of a fictitious lateral-spreading velocity was made to remedy that omission. The adequacy of the Prych simulation of ambient turbulence and shear stresses has not been. verified. Calibration of the model and its empirical coefficients with hydraulic or prototype data might have improved predictions.

As with the Stolzenbach-Harleman model, the Prych model is applicable for small or zero ambient currents due to the assumption of similarity for temperature and velocity profiles, as well as equal entrainment on offshore and lee sides of the jet. The computer code developed by Prych operates well (model equations are simpler than those of Stolzenbach and Harleman); this makes it easier for future alteration, manipulation, and calibration of the model. 


\section{RECOMMENDATIONS FOR FUTURE RESEARCH}

A. Field-data Acquisition

Other techniques, such as fixed instrument a rrays, aerial photography and aerial infrared imagery, and nonstationary measurement systems, may prove worthwhile in future measurements in the jet regime. These techniques or combinations of techniques may make it possible to overcome the limitations inherent in the fixed-boat method described here, but will probably also result in increased complexity of the measurement and significant monetary commitment.

B. Data-smoothing 'l'echnique

To extract ao much information ae posible from the data, the most general functional forms practicable should be used. This would require more free parameters and a more involved and lengthy titting process. Future attempts to extend this method of data analysis might include some of the following:

1. Instead of Gaussian lateral profiles, a function that allows for a flat region near the jet centerline might be chosen. This could then simulate the core region included in the Stolzenbach-Harleman analysis.

2. The form of the centerline temperature excess should be such as to require that it extrapolate to the measured excess at the outfall $(s=0)$. The present form does not have this property.

3. In the present procedure, the rate of dropoff of the centerline temperature and velocity excesses is fixed as being inversely proportional to the one-half power of $\mathrm{s}$, the distance from the outfall. Instead of this being restricted to the one-half power, it could be left as a free parameter to be determined by fitting to the data. The more recent phenomenological model by Pritchard ${ }^{24}$ has employed this form for centerline decay of temperature.

4. Additional parameters could be added to the expressions for the widths to allow them to vary quadratically with $\mathrm{s}$ instead of linearly. The alternatives are limitless, and only through repeated attempts at a fitting procedure can it be determined whether significant improvements are possible.

\section{Analytical Model; Field-data Comparisons}

1. An attempt should be made to calibrate the Motz-Benedict, Stolzenbach-Harleman, and Prych models to field and hydraulic data.* The Pritchard model (No. 1) should be further tested with field data from other sites as well as available data from physical hydraulic models. The new numerical models of Brady and Geyer, Paul and Lick, and Waldrop and Farmer

* Work is presently underway by Dr. M. Shirazi at the Pacific Northwest Environmental Research Laboratory at Corvallis to improve the Stolzenbach-Harleman and Prych models by calibration with data. At this writing, a successful modification and calibration of the Prych model appears imminent. 
look promising, and attempts should be made at verification with prototype field data. The new phenomenological model of Pritchard (No. 2), based upon 52 sets of model and prototype data, also looks promising and should be verified.

2. Considerably more data are required from more ideal or classical types of discharge structures for verification. The Palisades data had too many irregularities (rough shallow bottom and diverging discharge channel) for adequate model evaluation for those integral-type models studied in this report. Data are required for model verification (for both integral and numerical models), which include a wide range of densimetric Froude numbers, aspect ratios, bottom slopes, angle of discharge, ambient currents, etc., to provide a fair and wide variety of test situations for the models. Only when this large body of data (physical model or preferably prototype field data) becomes available will it be possible to fully and fairly evaluate and improve, or develop, better models.

From our verification efforts supplemented by the work done by Dr. M. Shirazi at the Pacific Northwest Water Laboratory of the EPA, we recommend that:

1. The Stolzenbach-Harleman and Prych models not be used as they exist in their present form for those cases when significant bottom interaction is expected. However, the vast majority of prototype situations do have some bottom interaction.

2. The Motz-Benedict model be used for stagnant ambient water case only (with an entrainment coefficient on the order of 0.04)

3. The Pritchard model be used for stagnant receiving water only.

Further analytical work is necessary to modify the Prych and StolzenbachHarleman models so they can be used for shallow water surface discharges. Additional work in model calibration is necessary before a viable form of the Motz-Benedict model can be achieved for ambient currents. More verification work on the Motz-Benedict (no current) and Pritchard models would be us eful. 


\section{APPENDIX A}

$\underline{\text { Previous Program Reports }}$

1. J. G. Asbury, Effects of Thermal Discharges on the Mass/Energy Balance of Lake Michigan, ANL/ES-1 (July 1970).

2. E. Silberman and H. Stefan, Physical (Hydraulic) Modeling of Heat Dispersion in Large Lakes: A Review of the State of the Art, ANL/ES-2 (Aug .17, 1970).

3. J. G. Asbury, R. E. Grench, D. M. Nelson, W. Prepejchal, E. P. Romberg, and P. Siebold, A Photographic Method for Determining Velocity Distributions within Thermal Plumes, ANL/ES-4 (Feb 1971).

4. J. G. Asbury and A. A. Frigo, A Phenomenological Relationship for Predicting the Surface Areas of Thermal Plumes in Lakes, AN L/ES-5 (Apr 1971).

5. I. K. Abu-Shumays, D. L. Phillips, and S. M. Prastein, "Thermal Plume Data Acquisition, Documentation and Initial Analysis," Proceedings of the 14th Conference of the International Association of Great Lakes Research, Toronto, Ontario, April 19-21, 1971, p. 495.

6. G. P. Romberg, W. Prepejchal, and D. M. Nelson, "Thermal Plume Measurements," Proceedings of the 14th Conference of the International Association of Great Lakes Research, Toronto; Ontario, April 19-21, 1971, p. 625.

7. G. E. Birchfield, Wind-driven Currents in a Large Lake or Sea, ANL/ES-6 (July 1971).

8. J. V. 'l'okar; 'Thermal Plumes in Lakes: Compilations of Field Experience, ANL/ES-3 (Aug 1971).

9. R. E. Nakatani, D. Miller, and J. V. Tokar, "Thermal Effects and Nuclear Power Stations in the U.S.A.," International Atomic Energy Agency Transactions, Vienna, 1971, IAEA-SM-146/30, p. 561.

10. J. G. Asbury and A. A. Frigo. "A Phenomenological Relationship for Predicting the Surface Area of Thermal Plumes in Lakes," Transactions of the American Nuclear Society, 1971 Winter Meeting, Notnher 17=2.1, 1971, Volume 14, No. 2, p. 461 .

11. B. M. Hoglund, D. Nelson, and S. Spigarelli, "The Anatomy of a Thermal Plume and its Biological Implications," Transactions of the American Nuclear Society, 1971 Winter Meeting, October 17-21, 1971, Volume 14, No. 2, p. 462.

12. A. J. Policastro and J. V. Tokar, Heated Effluent Dispersion in Large Lakes: State- - of-the-Art of Analytical Modeling: Part 1. Critique of Model Formulations, ANL/ES-11 (Jan 1972). 
13. T. H. Hughes and G. E. Birchfield, A Compilation of the Average Depths of Lake Michigan and Lake Ontario on a Two-minute Grid, AN L/ES-10, (Jan 197.2).

14. J. E. Draley, The Treatment of Cooling Waters with Chlorine, AN L/ES-12 (Feb 1972).

15. A. A. Frigo, "Prediction of Surface Plume Areas Associated with Heated Discharges into Large Lakes--A Phenomenological Model," Proceedings of the 15 th Conference of the International Association of Great Lakes Research, Madison, Wisconsin, April 5-7, 1972, p. 583.

16. B. M. Hoglund and S. A. Spigarelli, "Studies of the Sinking Plume Phenomenon," Proceedings of the 15th Conference of the International Association of Great.Lakes Research, Madison, Wisconsin, April 5-7, 1972, p. 614 .

17. A. J. Policastro, "State-of-the-Art of Analytical Modeling of Heated Effluent Dispersion in Large Lakes," Proceedings of the 15 th Conference of the International Association of Great Lakes Research, Madison, Wisconsin, April 5-7, 1972, p. 652.

18. Center for Environmental Studies and Environmental Statement Project, Summary of Recent Technical Information Concerning Thermal Discharges into Lake Michigan, Argonne National Laboratory for the Environmental Protection Agency Region V, Enforcement Branch, Contract Report 72-1 (Aug 1972).

19. A. A. Frigo and D. E. Frye, Physical Measurements of Thermal Discharges into Lake Michigan: 1971, ANL/ES-16 (Oct 1972).

20. A. J. Policastro, "Heated Effluent Dispersion in Large Lakes: State-ofthe-Art of Analytical Modeling, Surface and Submerged Discharges," paper presented and published in Session Notes of the Topical Conference, Water Quality Considerations: Siting and Operating of Nuclear Power Plants, Atomic Industrial Forum, Inc. (Oct 1972).

21. A. J. Policastro and R. A. Paddock, Analytical Modeling of Heated Surface Discharges with Comparisons to Experimental Data, paper presented at the 1972 Annual Meeting of AIChE, November 26-30,1972, to be published (Heat Transfer Symposium Series, 1973).

22. A. J. Policastro, Thermal Discharges into Lakes and Cooling Ponds, paper presented at ASCE Water Resources Conference, Washington, D.C. (F eb 1973).

23. R. A. Paddock, J. V. Tokar, and A. J. Policastro, "Analys is of Data Taken in the Near-Field Region of a Surface Thermal Discharge with Comparisons to Analytical Model Predictions, "paper presented at 16 th Conference of the International Association of Great Lakes Research, Huron, Ohio (Apr 1973). 
24. D. E. Frye, A. A. Frigo, and B. M. Hoglund, "Data Collection and Reduction Techniques Used for Investigating Thermal Discharges," paper presented at 16 th Conference of the International Association of Great Lakes Research, Huron, Ohio (Apr 1973).

25. A. A. Frigo; D. E. Frye, and P. Siebold, "Temperature and Velocity Measurements in the Near-Field Region of Thermal Plumes," paper presented at 16 th Conference of the International Association of Great Lakes Research, Huron, Ohio (Apr .1973).

26. A. J. Policastro and W. Dunn, Chapter 13: "Heated Surface Discharges-Mathematical Models and Similarity Principles," Chapter 14: "Heated Surface Discharges--Application and Verification of State-of-the-Art Near-Field Models," Heat Disposal in Power Plant Siting, Joint Center for Graduate Study; Richland, Wash., August 20-24, 1973. 
APPEN DIX B

Preliminary Feasibility Study.

On November 3, 1971, a preliminary feasibility study of the technique for studying the temperature and velocity profiles was made near the outfall of the Point Beach Nuclear Power Plant (Unit 1). A three-point mooring system was used to hold the boat steady while simultaneous temperature and velocity measurements were obtained in the near-field region. Anchors were located on either side of the plume and attached to the stern cleats of the boat, and a bowline was attached to the center of the outfall. Transectsacross the plume centerline were then made at about 8,27 , and $73 \mathrm{~m}$ from the outfall by pulling the boat from one side anchor to the other. The position of the boat was held relatively constant, and transits were used to obtain the location of each station. (Station locations for this jet study are shown in Fig. 94.) A Bendix Q-15 current meter with a YSI thermistor attached was used to measure current velocity and water temperature. The meter was lowered over the side and suspended at 2 - $\mathrm{ft}$ intervals to a depth of $10 \mathrm{ft}$ or to the bottom. The lake depth was $4.1 \mathrm{~m}$ at the outfall and decreased in depth to $2.1 \mathrm{~m}$ at a point $73 \mathrm{~m}$ from the outfall.

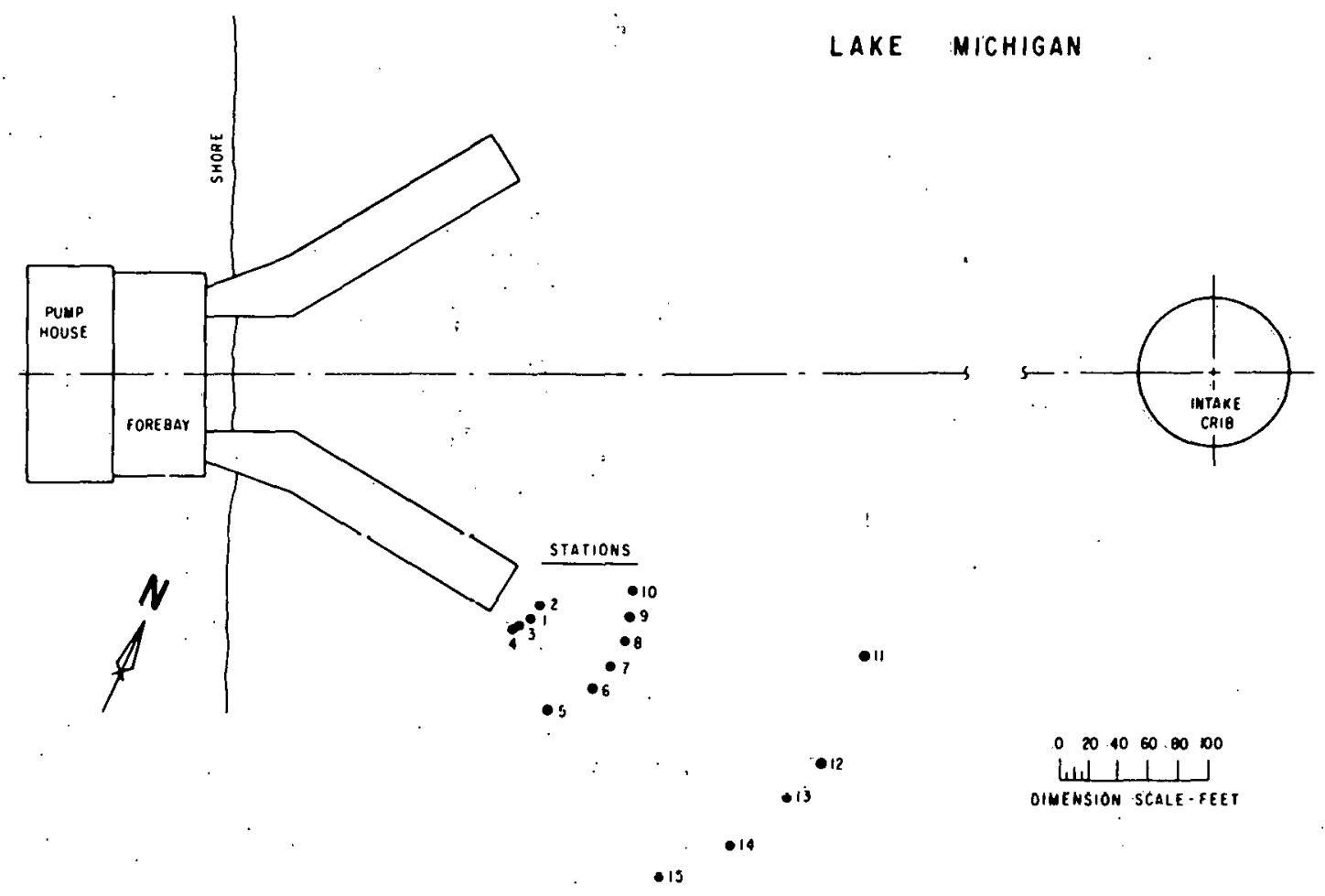

Fig. 94. Station Locations for Jet-regime Study: Nuvember 3, 1971, 1245-1605 Hours. ANL Neg. No. 190-573.

The data with the range of variability are plotted in Figs. 95-97 for three different depths. Drawings for the 8 - and $10-\mathrm{ft}$ depths were not made because data were not available at all stations. The temperature at a given 

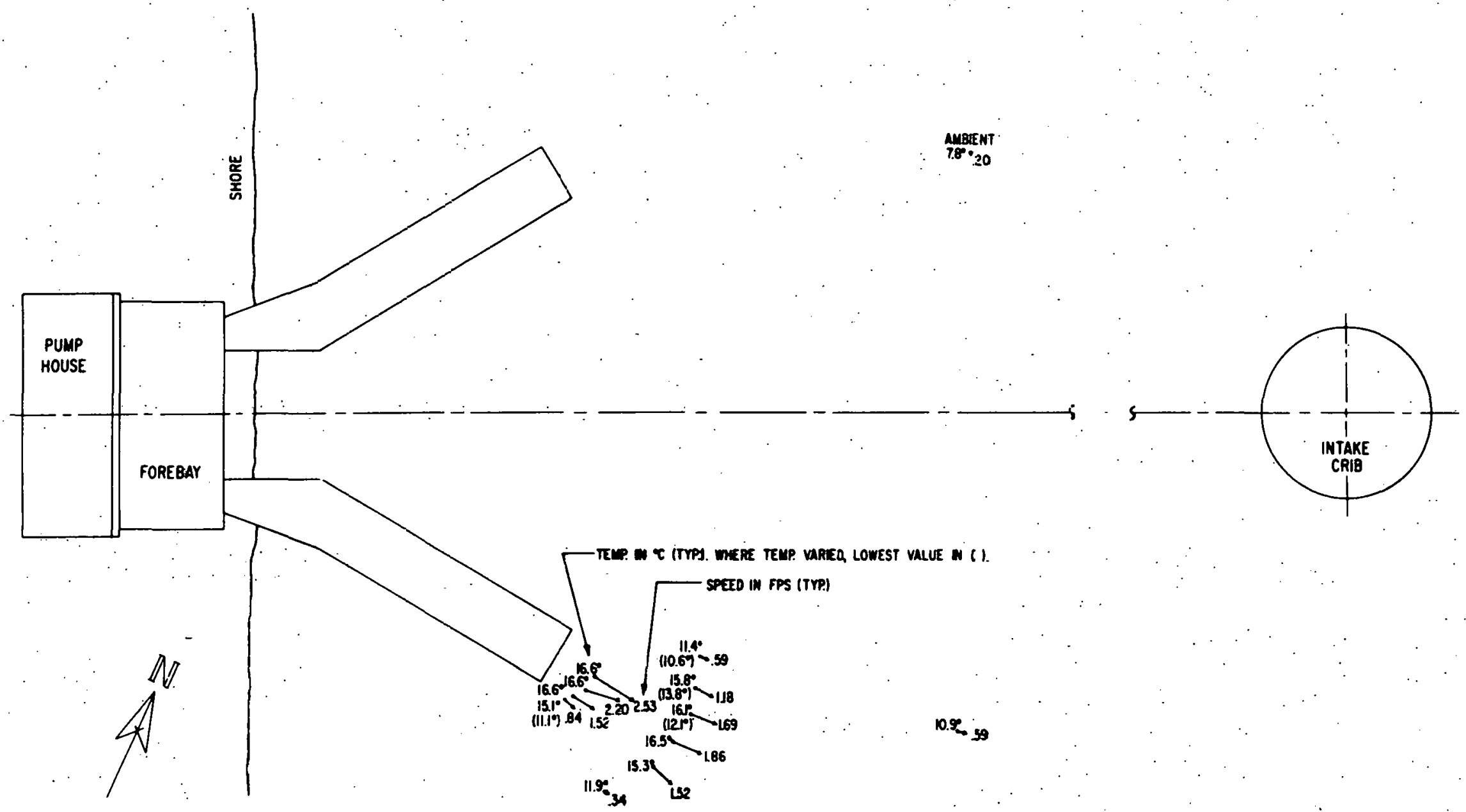

PLANT \& METEOBOLOGKCAL DATA PLANT NAME: POINT BEACH MUCLEAR PLANT (UNIT 1$)$
OATE \& TIME OF SURVEY: O3 NOV 71 (124S-1605) PLANT LOAD (MWO): 480

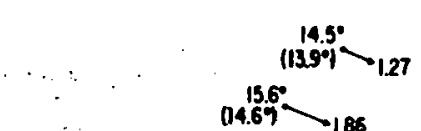

OUTFALL TEMPERATURE: $16,7.7 \mathrm{C}$ INTAKE TEMPERATURE: $7.8^{-0.33^{\circ} \mathrm{C}}$ ORY GULB TEMPERATUAE: $3.3 .4 .44^{\circ} \mathrm{C} C$

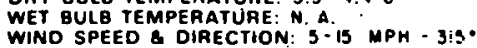
WIND SPEED D DIATCTON: 3 - 15 MPH - 390. LARE LEVEL: 579.3 FT. TIGLO
LAKE SURFACE CONDITONS 0.1 FT. WAVES

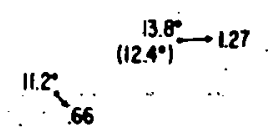

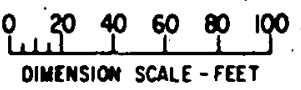

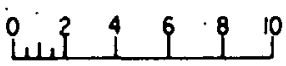

VELOCITY SCML - FPS

Fig. 95. Jet-regime Study for $2-f_{1}$ Depth at Point Beach Power Plant (Unit 1): November 3, 1971, 1245-1605 Hours. A.NL Neg. No: 190-410. 


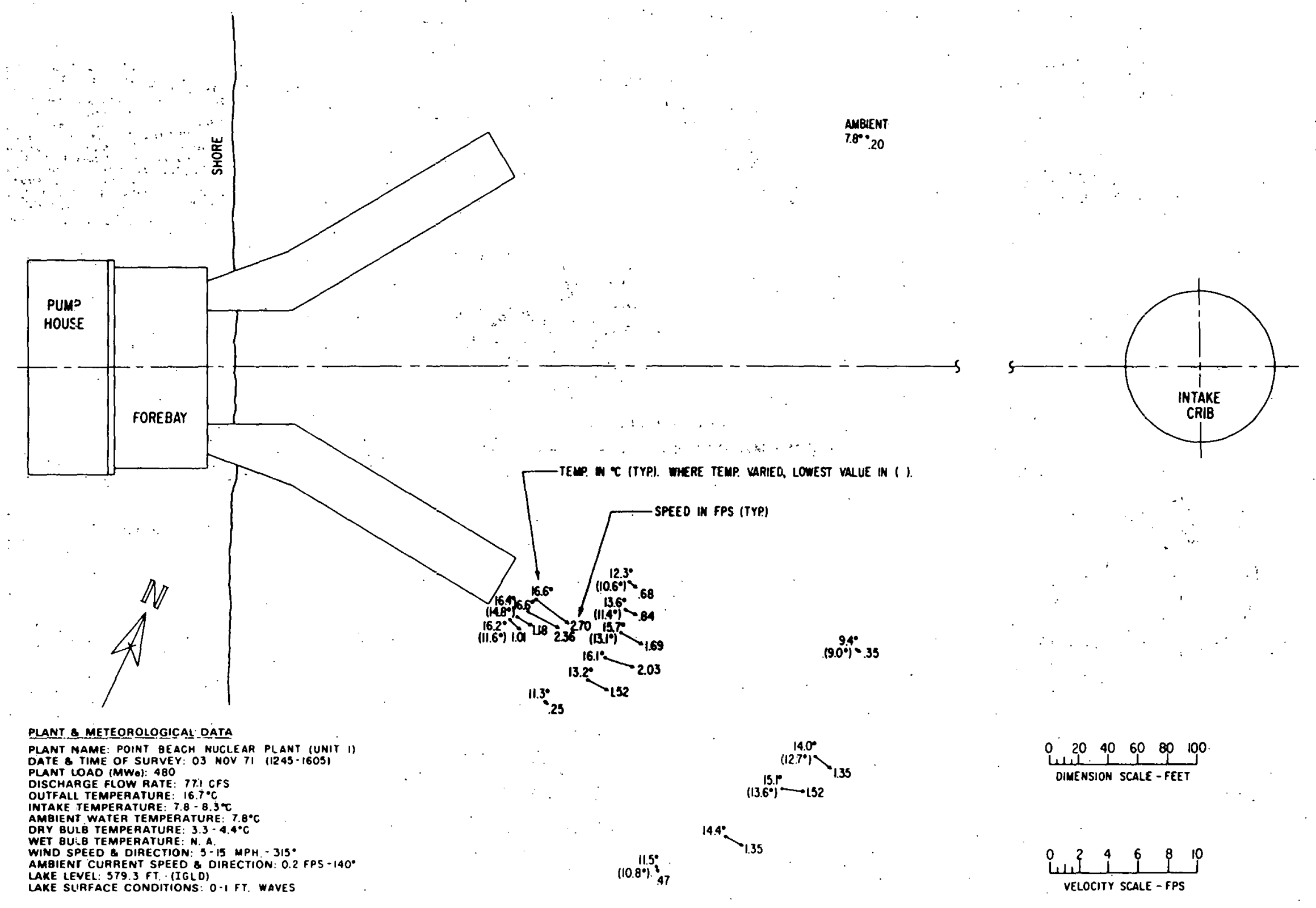

Fig. 96. Jet-regime Study for 4 -ft Depth at Point Beach Power Plant (Unit 1): November 3, 1971, 1245-1605 Hours. ANL Neg. No. 190-411. 


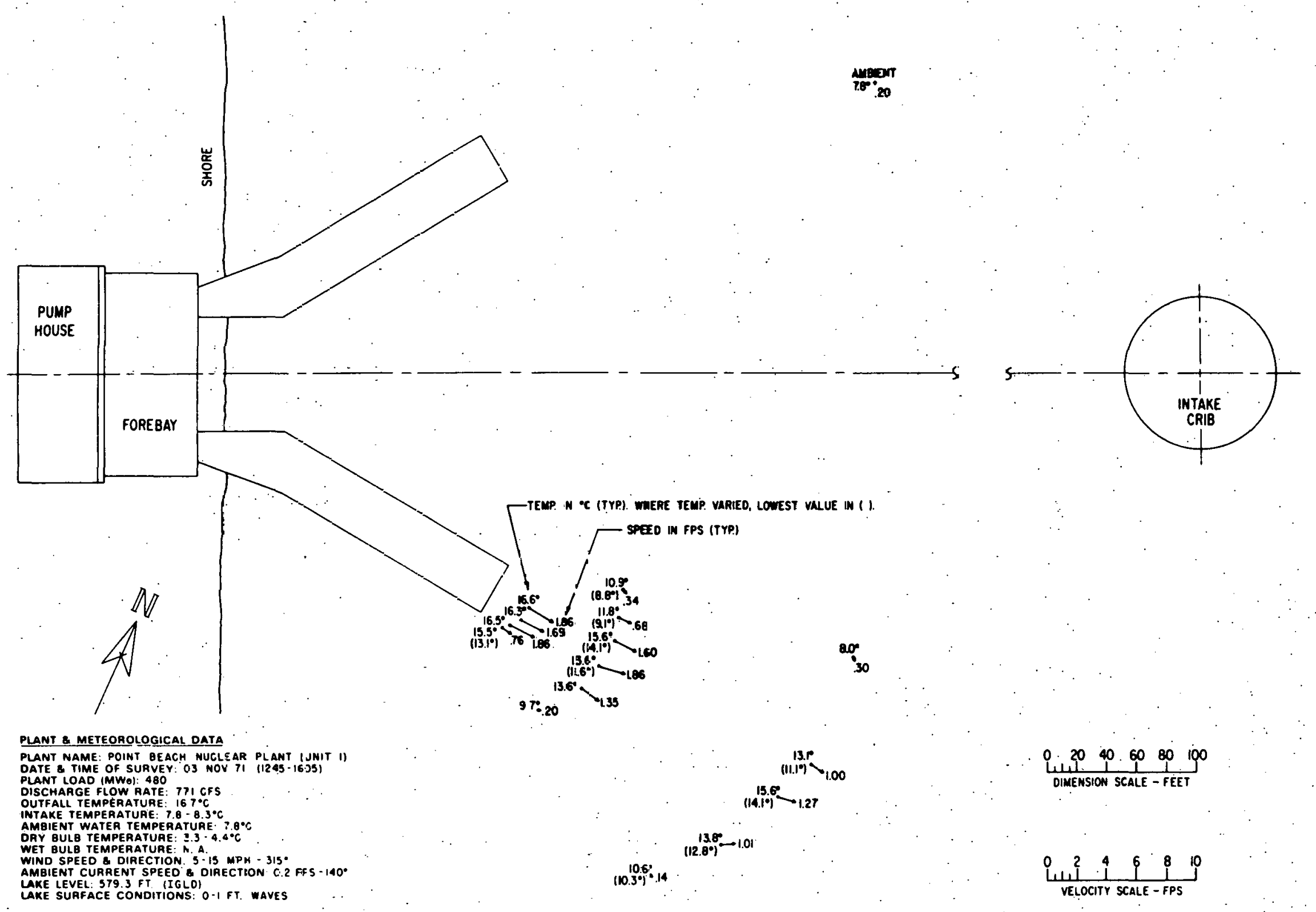

Fig. 97. Jet-regime Study for 6-ft Depth at Point Beach Power Plant (Unit.1): November $3,1971,: 245-1 € 05$ Hours. ANL Neg. No. 190-412. 
depth and location exhibited greater variability than expected, even near the plume centerline. As a result, temperature ranges are presented in the figures with the lowest temperature-in parentheses. Each measurement was made over a period of about $1 \mathrm{~min}$, which is indicative of the rapid variability of the temperature. Variations in the velocity were not as easily observed, because of the current-meter time constant. The lengths of the arrows in the figures are proportional to current magnitude, and their directions indicate current directions.

A more complete discussion of this jet-regime study appears in Ref. 19 of Appendix A. The results of this preliminary study are presented here only for completeness and were not used in the analysis discussed. In fact, since all the data were collected within $75 \mathrm{~m}$ of the outfall, the data are more representative of the flow-establishment region than of the established flow regime of the jet. 


\section{APPENDIX C \\ FORTRAN Listing for Fitting Procedure}

The FORTRAN listing of the computer code JETFIT used for the jet data fitting procedure appears below.

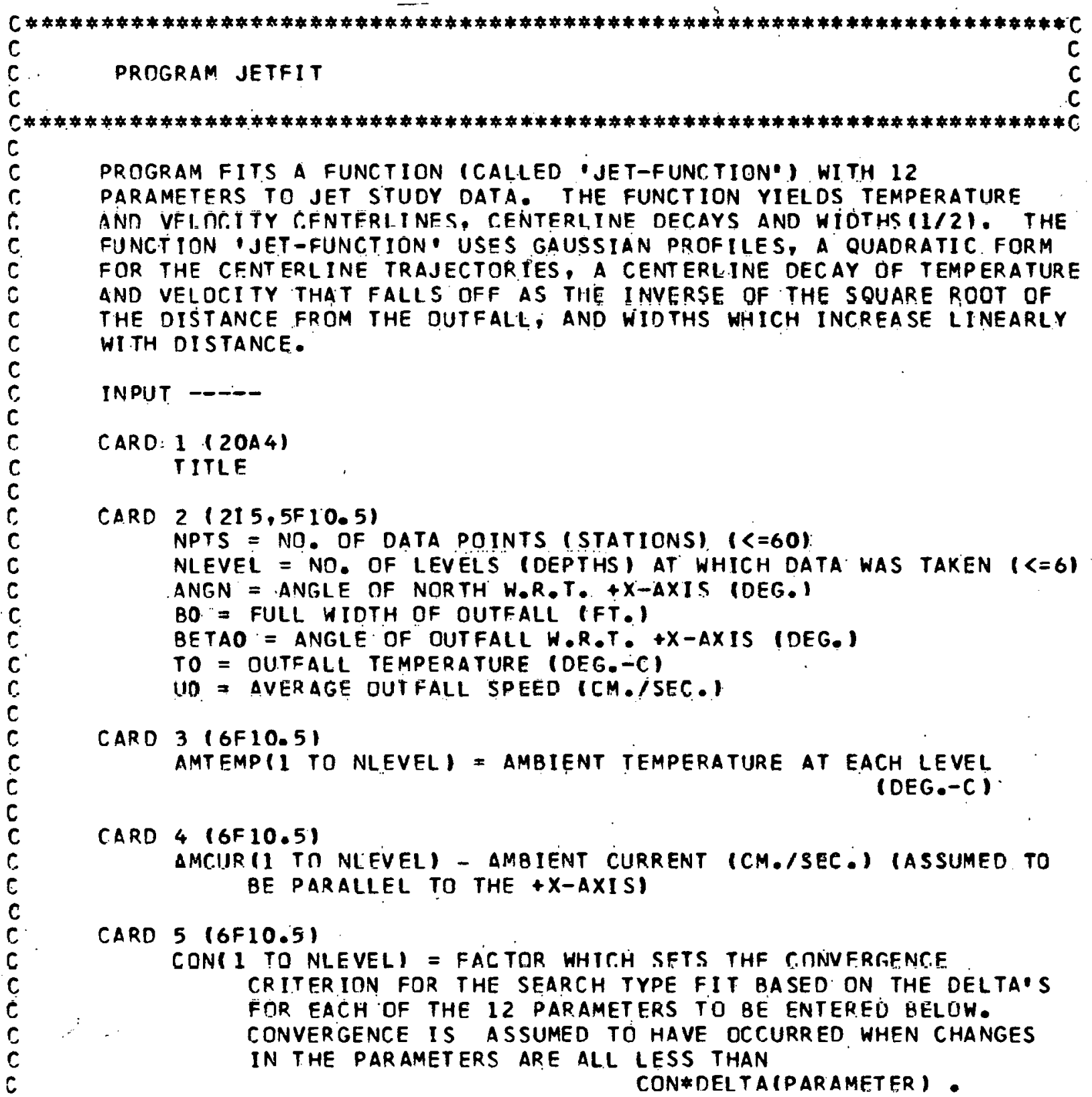

CARD $6(6 F 10.5)$

ALIMTII TO NLEVELI = MAXIMUM NUMBER OF ITERATIONS IN FITTING THE TEMPERATURE PART OF 'JET-FUNCTION' ISTEPS ARE PRINTED OUT IF ALIMT $<0.01$.

CARD 7 (6F 10:5)

ALIMVII TO NLEVELI = SAME AS ABOVE BUT FOR THE VELOCITY PART OF THE FUNCTION.

CARD 8 (6F10.51 


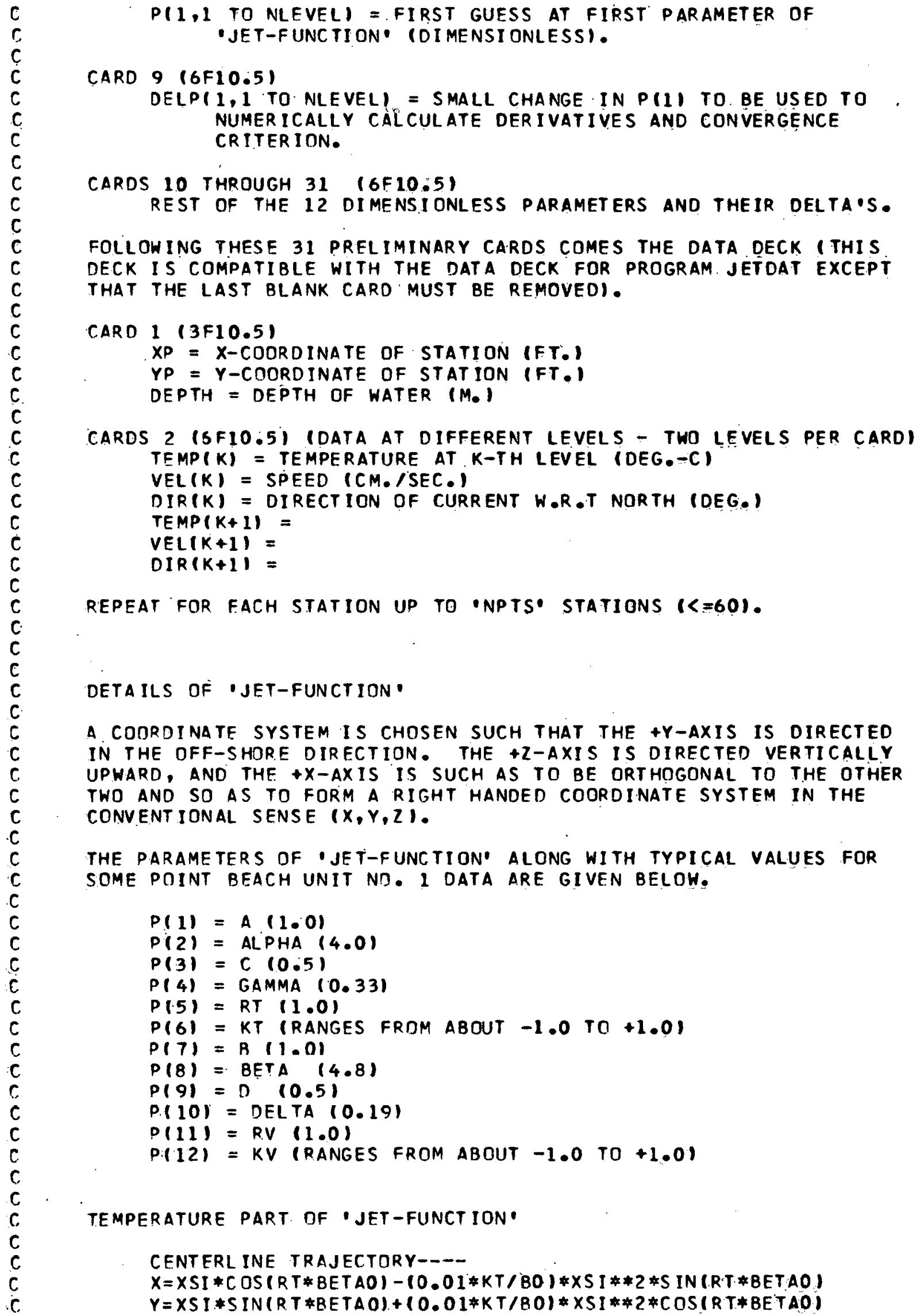


WHERE XSI IS INTROOUCED ONLY DUE TO THE PARAMETRIC FORM OF THE EQUATIONS (BENDS LEFT FOR KT $>0$, BENDS RIGHT FOR $K T\langle O)$

CENTERLINE TEMPERATURE EXCESS RATIO----

(TC-TA)/(TO-TA) $=A$ IF S $\angle A L P H A * B O$ $=A * S Q R T$ ( ALPHA*BO/S) IF S $>A L P H A * B O$.

WHERE S IS THE DISTANCE FROM THE OUTFALL MEASURED. ALONG THE CENTERL INE

TEMPERATURE WIDTH (TO $1 / 2$ THE CENTERLINE VALUE) - WT /BO $=$ C +GAMMA*S/BO

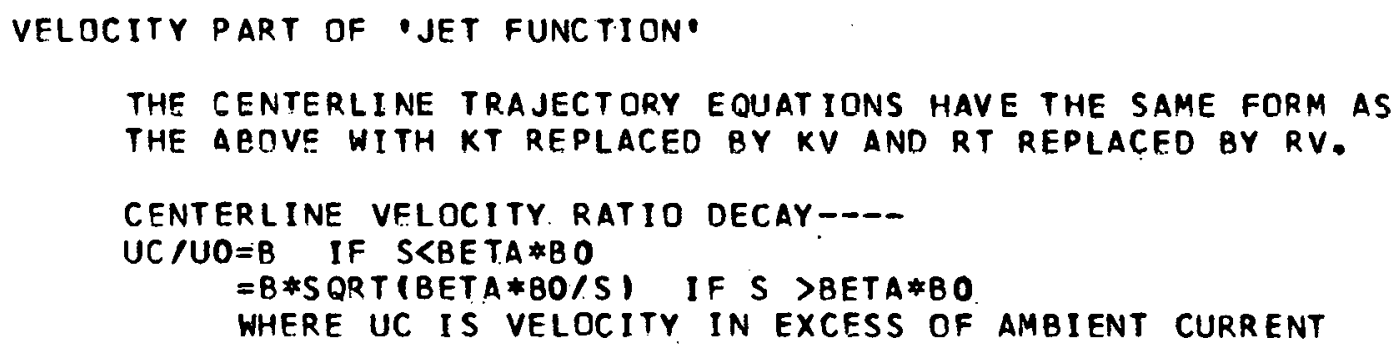

DIMENS IDN TITLE (20), CON (6), ALIMT( 6), ALIMV(6),P(12,6), DEPTH(60),BTI (6), BV (6), EPSB(6), AREA(6)

COMMON/HAVE /DELP $(12,6)$, TEMP $(60,6)$, VEL $(60,6), D I R(60,6), X P(60), Y P(60$

1), AMTEMP ( 6 ), AMCUR (6), BETAO,BO, ANGN, TO, UO,NPTS,OR, RO, K

EXTERNAL FTSIG

EXTERNAL FVSIG

$D R=0.0174532925$

$R D=57.295780$

IMAX $=27$

c 
96

97

98

99

900

901

902

903

904

905

906

907

908

909

910

911

912

913

914

915

.916

917

918

019

920

921

922

923

C

C

33

1

FORMAT $(4 X, I 2,5 X, F 7.2,6 x, F 8.2,6 x, F 8.5,6 x, F 7.1,5 X, F 7.11$ FORMAT $/ 1 /, 1$ INITIAL PARAMETERS OF JET-FUNCTION IALL ARE DIMENSION

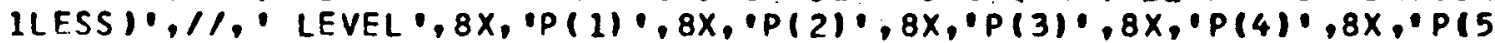
$21 \cdot, 8 \times, \cdot P(6) \cdot, / 1)$

FORMAT $(4 x, 12)$

FORMAT $(6 X, 6(2 X, F 10.6))$

FORMAT $\left(1 / 0^{\circ}\right.$ LEVEL',8X, $P(7) \cdot, 8 X, \cdot P(8) \cdot, 8 X, \cdot P(9) \cdot, 7 X, \cdot P(10) \cdot, 7 X, \cdot P($ $1111 \cdot 7 \times, \cdot P(12) \cdot, 1)$

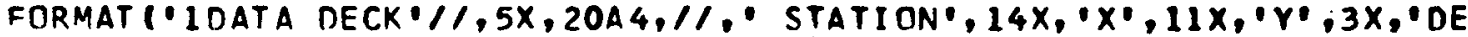

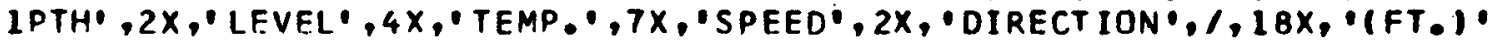

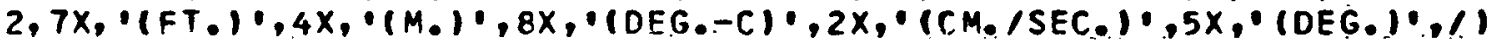
FORMAT $15 x, 13,5 x, F 10.2,2 x$, F10:2, 2X, F6.2)

FORMAT $(48 \mathrm{X}, 12,5 \mathrm{x}, \cdots-\cdots, 8 \mathrm{x}, \cdots-\cdots+7 \mathrm{X}, \cdots-\cdots)$

FORMAT $(48 X, 12,2 X, F 7.2,5 X, F 7,2,4 X, F .7 .2)$

FORMAT 1'1LEVEL ', I2,3X, 'ITEMPERATURE FIT $11, / 1,5 \mathrm{X}, 20 \mathrm{~A} 4,1 / 1$

FORMAT/ $/ /, \cdot$ FINAL TEMPERATURE RESULTS FOR',12,'-TH LEVEL $, 1 /, 8 \mathrm{X},{ }^{\prime} \mathrm{S}$

1 IGMA $=?$, F8.3,' AFTER $1,16,1$ ITERATIONS.', $/ 11$

FORMATIIOX, P(०, $12, \cdot 1)=\cdot, F 10.6)$

FDRMATI'ICOMPARISON OF DATA AND FIT RESULTS FOR THE', I2, -TH LEVEL

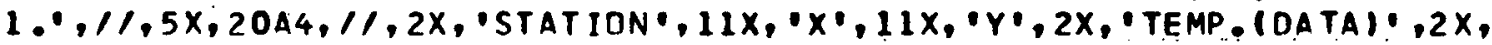
2'TEMP. (CALC. $1 \cdot, 3 X$, BETAT (CALC.)',2X, 'SPEED (DATA) , $2 X$, 'SPEEDICALC.)

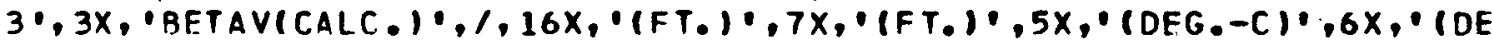

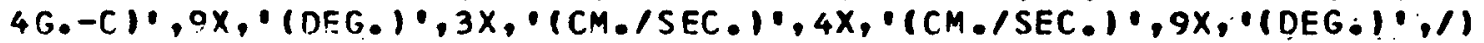
FORMAT $(6 x, 13,2 X, F 10.2,2 X, F 10.2,6 x, F 7.2,7 X, F 7,2,8 X, F 7.2,6 x, F 7,2,7 X$, $1 F 7.2,8 X, F 7.21$

FORMAT (//,' TABLE OF JET-FUNCTION (TEMPERATURE PART) $1,1 /, 9 x, \cdot x S I \cdot$,

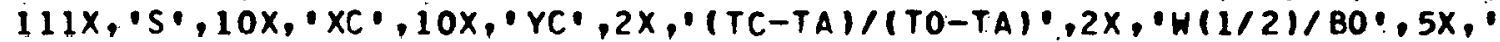

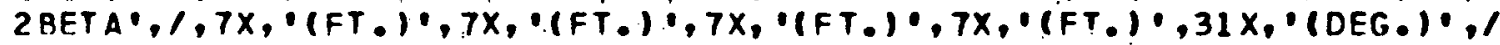
3)

FORMATI $2 X, F 10.2,2 X, F .10 .2,2 X, F 10.2,2 x, F 10.2,9 X, F 8,4,3 x, F 8,3,2 x, F 7$. 121

FORMAT $/ / /, 1$ TABLE OF JET-FUNCTION (VELOCITY PART) $, / /, 9 X, \cdot X S I, 11 X$

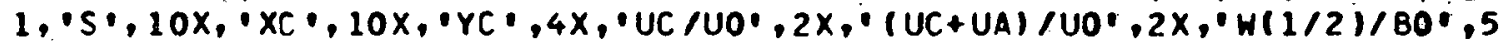

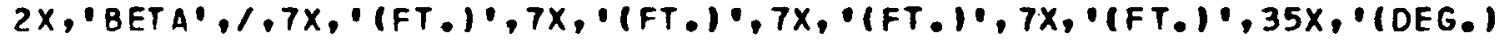
$3 \cdot, 1)$

FORMAT $12 X, F 10.2,2 X, F 10.2,2 X, F 10.2,2 x, F 10.2,2 X, F 7.4,4 X, F 8.4,3 X, F B .3$

$1,2 \times, F 7.21$

FORMATI/, TDO MANY DATA POINTS., $/ 1$

FORMAT $(1,-$ TOO MANY LEVELS.,$/ /)$

FORMAT $(\cdot 1$ LEVEL $, 12,3 X, \cdot($ VELOCITY FIT $), / 1,5 X, 20 A 4,1 / 1)$

FORMATI//, FINAL VELOCITY RESULTS FOR', I2, '-TH LEVEL', $1 /, 8 X$,

I'S IGMA $=1$, F8.3, - AFTER', I6, - ITERATIONS.', //I

FORMATI'IAPPRDXIMATE EXCESS TEMPERATURE ISOTHERM AREAS AND JET DEP 1 THS. $\because 1 /, 5 X, 20 A 4,1 / 1,2 x$, TEMP. EXCESS , 6X, 'AREA(ACRES) - $L$

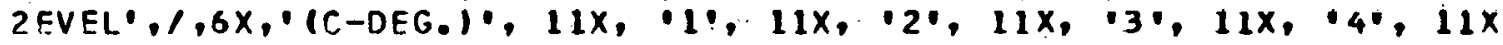
$3,15 \cdot 11 x, \cdot 6 \cdot, 11$

FORMAT $(7 X, F 7.2,6(2 x, F 10.4))$

FORMAT $/ / / /, 11 X$, S, $2 X$, TTEMP. EXCESS, $4 X$, DEPTH', 6X, 'ETALF

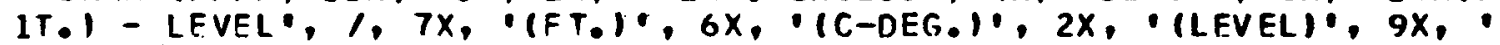
$21^{\prime}, 9 x, \cdot 2^{\prime}, 9 x, 13^{\prime}, 9 x, 14^{\prime}, 9 x, 15 \cdot, 9 x, 16^{\prime}, 11$

FORMAT $(2 X, F 10.2,7 X, F 7.2,2 X, F 7.3,6(2 x, F 8.2))$

FORMATI///, $11 X$, 'S', $3 x$, 'VEL. EXCESS', 4X, 'DEPTH', 6X, 'ETALFT 1.) - LEVEL', /, 7X, '(FT.)', 4X, '(CM./SEC.)', $2 x_{,}$, (LEVEL), $9 x_{\text {, }}$ $2^{\prime} 1 \cdot, 9 x, \cdot 2 \cdot, 9 x, 130,9 x, 140,9 x, 151,9 x, 161,11$

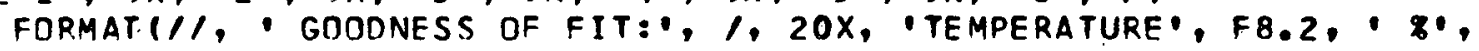
$1 /, 20 X$, VELLCITY', F11.2, $\$ 1$

READ IN DATA

READ $(5,90, E N D=30)(T$ ITLE $(1), I=1,20)$

READ $(5,91)$ NPTS, NLEVEL, ANGN,BO,BETAO;TO,UO

IF (NPTS -60$) 1,1,31$

IF (NLEVEL -6$) 7,7,32$ 


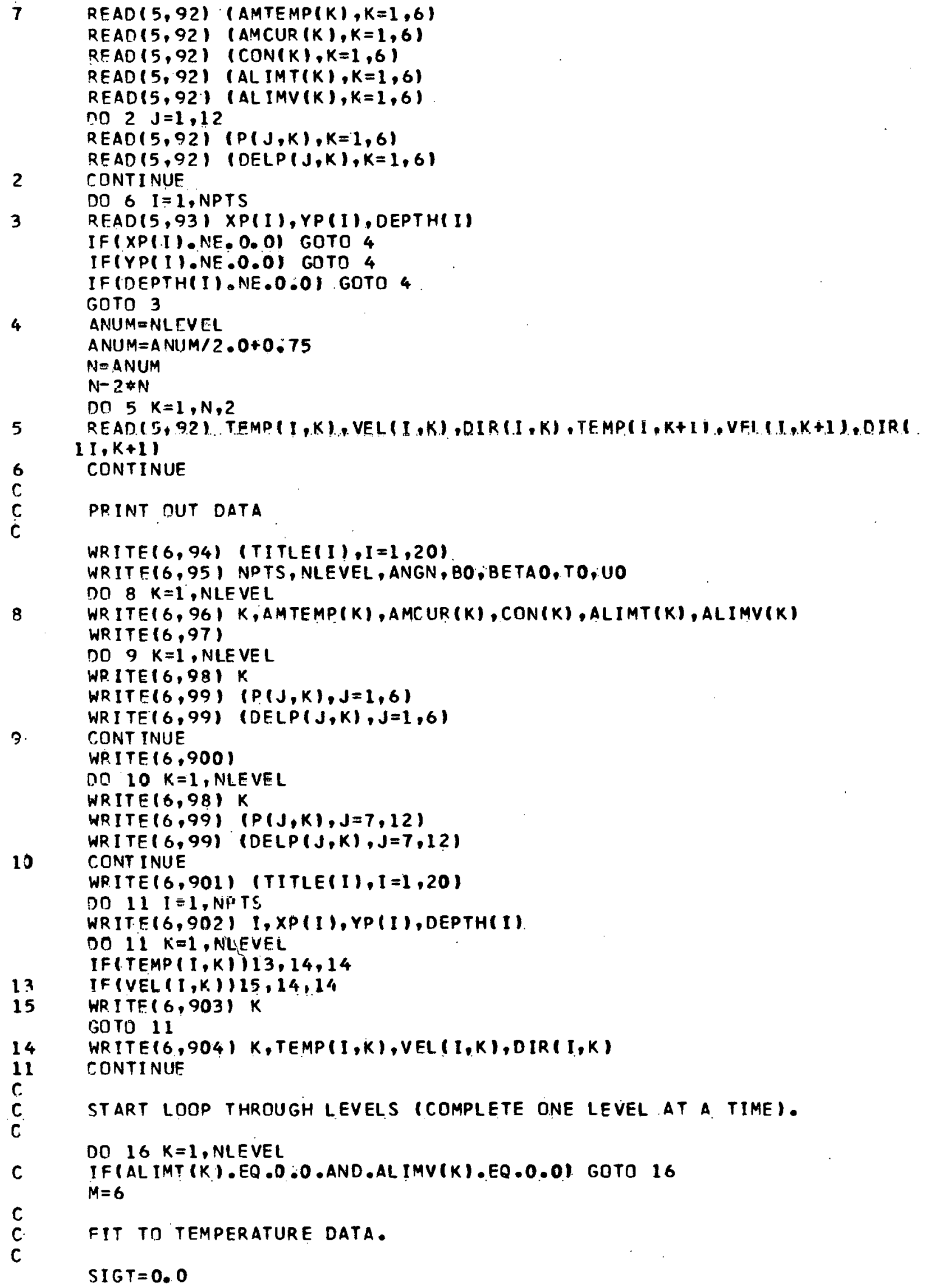


DELMAX $=0.0$

DO $17 \mathrm{~J}=1, M$

$B T(J)=P(J, K)$

$D E L M A X=D E L M A X+D E L P(J, K)$

17

EPSB $(J)=D E L P(J, K) * \operatorname{CON}(K)$

DELMAX $=0.16666667 *$ DELMAX

$L=A L I M T(K)$

C IF(L.EQ.0) GOTO 12

WRITE $(6,905) \quad K,(T I T L E(I), I=1 ; 20)$

CALL GMIN (FTSIG,M, BT, S IGT, EP SB, L, ITER, DELMAX)

CALL LABEL (ITER)

WR. ITE $(6,906) \mathrm{K}$, SIGT,L

DO $18 \mathrm{~J}=1, M$

$P(J, K)=B T(J)$

18 WR.ITE $(6,907) \mathrm{J}, \mathrm{BT}(\mathrm{J})$

WR ITE $(6,910)$

DD $24 I=1$, I MAX

ANUM $=1-1$

$X S I=A N U M * 50.0$

$\triangle N U M=0.01 * B T(6) / B O$

THETA $=D R * B T(5)$ \&RE TAO

$S T H=S I N(T H E T A)$

$C T H=C O S(T H E T A)$

$A I=2.0 * \times S I * A B S(A N U M)$

IF $(A 1-0.1) 34,34,35$

34

$S=X S I *(1.0+0.166666667 * A 1 * A 1-0.025 * A I * 4)$

GOTO 36

$35 \quad A 2=S Q R T(1,0+A 1 * A 1)$

$S=0.5 * X S I *(A 2+A L O G(A 1+A 2) / A I)$

$36 \quad X=X S I * C T H-A N U M * X S I * X S I * S T H$

$Y=X S I * S T H+A N U M * X S I * X S I * C T H$

$T=B T(1)$

IF $(S-B T(2) * B 0) 25,25,26$

$26 \quad T=T * S Q R T(B T(2) * B O / S)$

$25 \quad A 1=S T H+2.0 * A N U M * X S I * C T H$

$A 2=C T H-2,0 * A N U M * X S 1 * S T H$

$B E T A T=R D * A T A N 2(A), A 2)$

$W B O=B T(3)+B T(4) * S / B O$

24 WRIITE $(6,911)$ XSI,S,X,Y,T, WBO,BETAT

C

c

C

12

FIT TO VELOCITY DATA.

SIGV $=0.0$

DELMAX $=0: 0$

$0019 J=1, M$

$B V(J)=P(J+M, K)$

DELMAX $=D E L M A X+D E L P(J+M, K)$

$19 \operatorname{EPSB}(J)=D E L P(J+M, K) * C O N(K)$

DELMAX $=0.16666667 *$ DELMAX

$L=A L I M V(K)$

C IFIL.EQ.0) GOTD 52

WRITE $(6,916) \mathrm{K},($ TITLE $(I), I=1,20)$

CALL GMINIFVSIG, M, BV, SIGV, EPSB, L, ITER, DELMAX I

CALL LABELIITER)

WRITE $(6,917) \mathrm{K}$, SIGV,L

DO $20 \mathrm{~J}=7,12$

$P(J, K)=B V(J-M)$

20: WRITE $(6,907)$ J,BV(J-M)

WRITE $(6,912)$

DO 27 I $=1$, IMAX

$\triangle N U M=1-1$

$X S I=A N U M * 50.0$ 


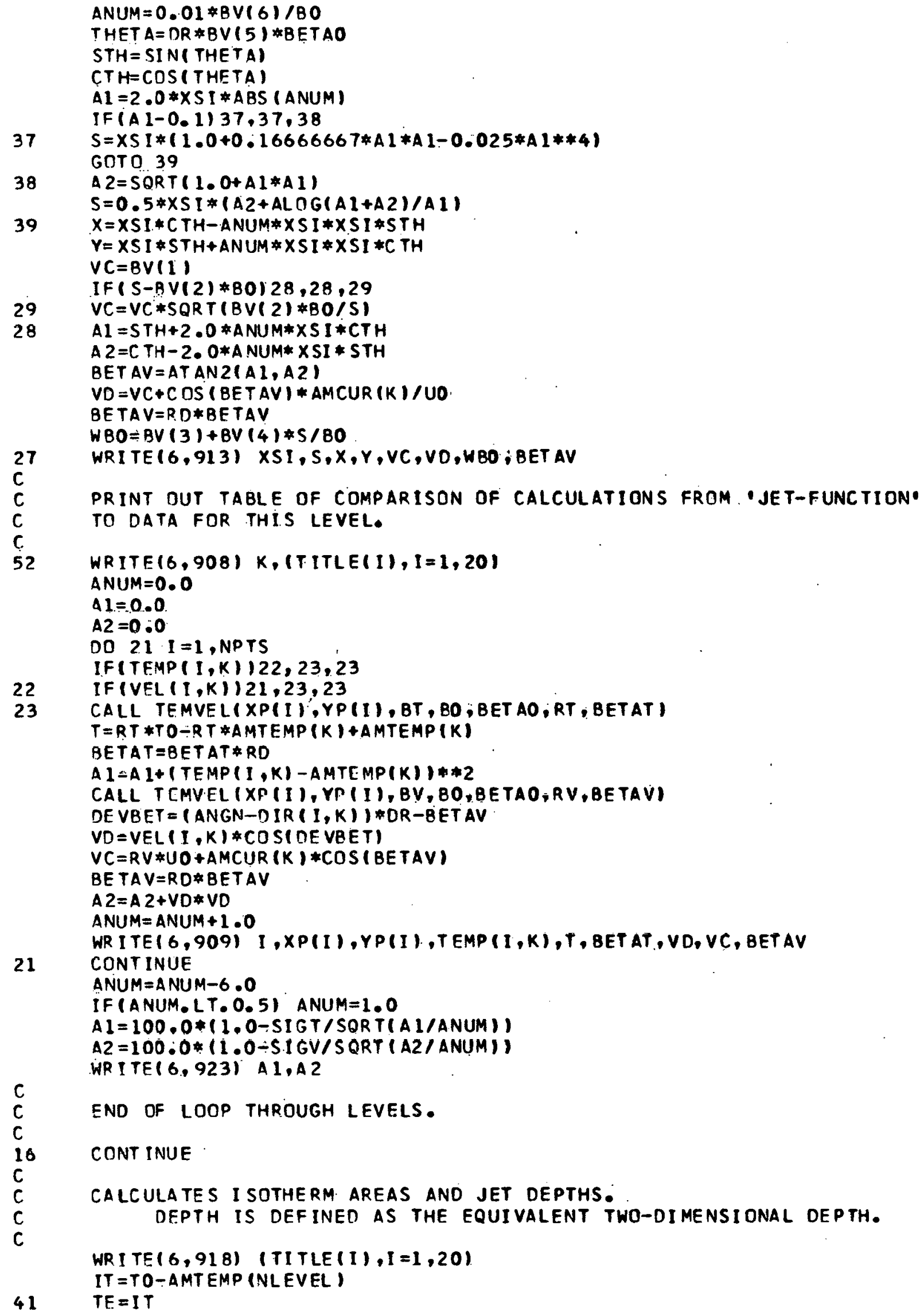


$\because$ DO $40 \mathrm{~K}=1$, NLEVEL

40

AREA $(K)=T A R E A(T E, P)$

WRI TE $(6,919)$ TE,. (AREA.(K), K $=1$, NLEVEL)

I $T=I T-1$

IF(IT.GE.I) GOTO 41

WRITE $(6,920)$

DO $42 I=1$, IMAX

ANUM $=1-1$

$S=50.0 *$ ANUM

$T=P(1,1) *(T O-A M T E M P(1))$

IF (S.LE.P $(2,1) * B O)$ GOTO 43

$T=T * \operatorname{SQRT}(P(2,1) * B O / S)$.

$43 \quad T=0.5 * T$

DO $44 K=1$, NLEVEL

$44 \quad A R E A(K)=E T A T(S, T, P)$

$\triangle I=0.0$

IF (ARE.A (1) . LT. $(0.1 * B O))$ GOTO 46

$A 1=0.5$ *AREA(1)

DO $45 \mathrm{~K}=2$, NLEVEL

$45 \quad A 1=A 1+A R E A(K)$

$A 1=A 1 / A R E A(1)+100$

46 WRITE $(6,921) S, T, A 2$, (AREA $(K), K=1$, NLE VEL)

42 CONTINUE

WR. I TE $(6,922)$

$\bullet$

DO $47 \mathrm{I}=1$, IMAX

$\triangle N U M=1-1$

$S=50.0 \div A N U M$

$V C=P(7,1) * U 0$

IF (S.LE.P $(8,1) * B O)$ GOTO 48

$48 \quad V D=0.5 * V C$

$V C=V C * S Q R T(P(8,1) * B O / S)$

DO $49 \mathrm{~K}=1$, NLEVEL

$49 \quad A R E A(K)=E T A V,(S, V D, P)$

$A 1=0: 0$

IF (AREA (1) . $(T,(0.1 * B 0))$ GOTO 51

$A 1=0.5 * A R$. EA (1)

DO $50 \mathrm{~K}=2$, NLEVEL

$50 \quad A 1=A 1+A R E A(K)$

$A 1=A 1 / \triangle R E A(1)+1.0$

51 WRITE $(6,921) S, V D, A 1$, (AREAIKI, $K=1$, NLEVEL $)$.

47 CONTINUE

C

GOTO 33

C

31 WR ITE(6,914)

GOTO 30

32 WRITE 6,915$)$

30 CALL EXIT

STOP

END

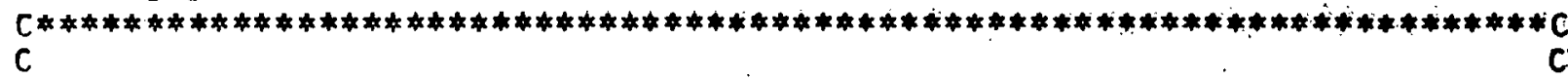

SUBROUTINE GMINIFUNC, $M, X, F$, EPS, LIIMIT, IT ER, DELMAXI:

$c$

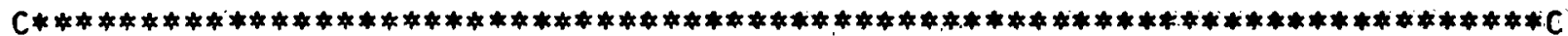

C

C ROUTINE FINDS THE MINIMUM BY A SEARCH TECHNIQUE OF THE

C FUNCTION (CALCULATED IN SUBROUTINE FUNCI WITH RESPEET TO ITS

C PARAMETERS $X(I), I=1, M_{0}$.. FIRST THE OIRECTION OF STEEPEST

C DECENT IS SELECTED BY EVALUATING THF GRADIENT OF THE. FUNCTION

C. WITH RESPECT TD THE M PARAMETERS. THEN A STEP IS TAKEN ALONG

C THAT DIRECTION. THE SIZE OF THE STEP IS PRESET BUT MULTIPLES OR. 


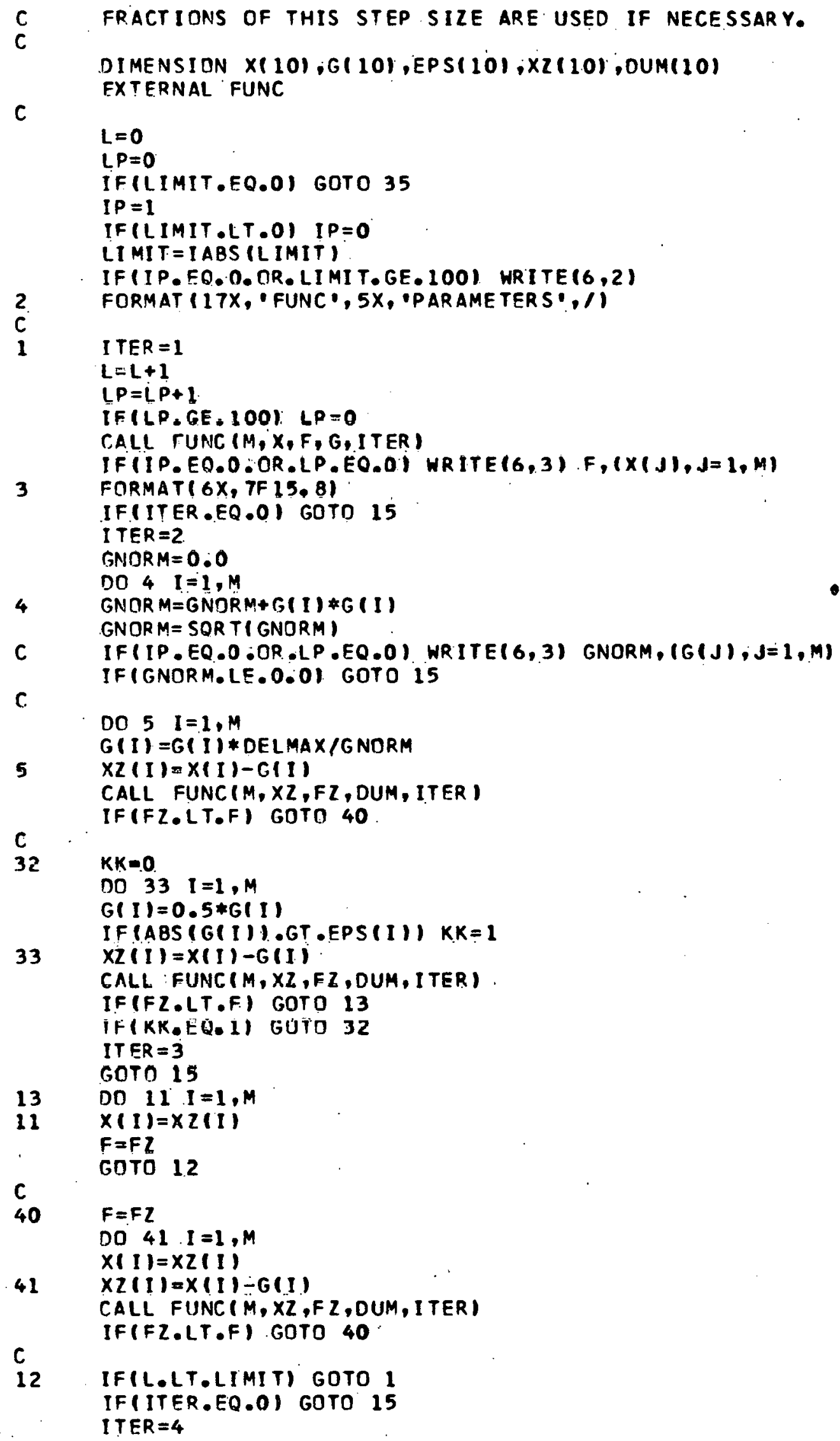




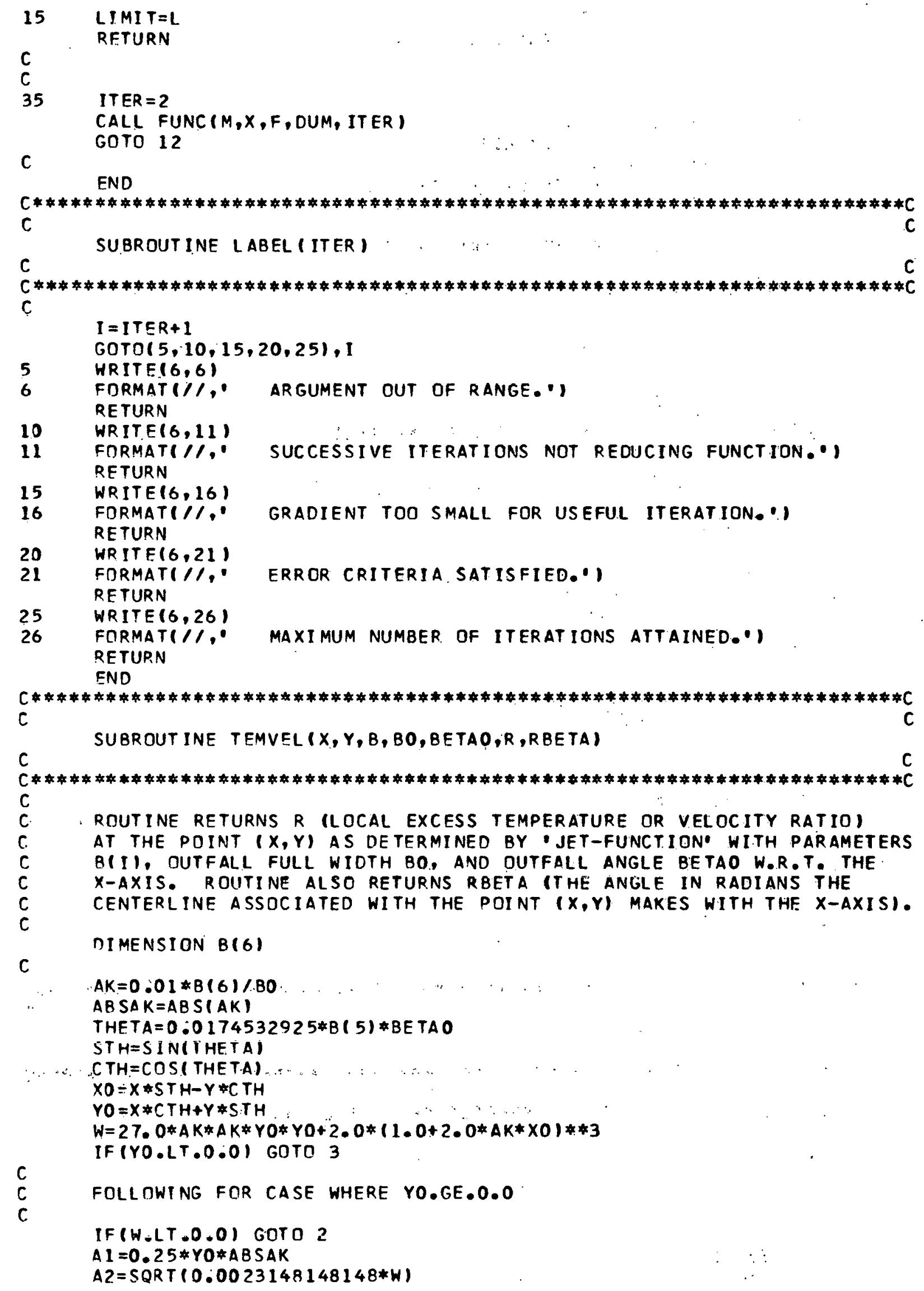


IF (A1 - LT.0.03*A2) GOTO 1 $X S I=(C U B R T(A 1+A 2)+C U B R T(A 1-A 2)) / A B S A K$

GOTO 4

$X S I=Y O * C U B R T(2.0 / W)$

GOTO 4

$2 \quad A 1=S Q R T(-1.0 \div 2.0 * A K * X 0)$

$S=(3.674234613 * Y 0 * A B S A K) /(A 1 * A 1 * A 1)$

$S=A R C O S(S) * 0.333333333$

$X S I=(0.8164965809 * A .1 * \operatorname{COS}(S)) / A B S A K$

GOTO 4

c

c.

3 IF(W.GT.0.0). GOTO 12

GOTO 2

NOW HAVE VALUE FOR XSI.

$4 \quad A L=2,0 * \times S I * A B S A K$

IF (A) $1-0.11) 5,5,6$

$5 S=X S I *(1.0+0.166666667 * A 1 * A 1-0.025 * A 1 * * 4)$

GOTO 7

$6 \quad A 2=S Q R T(1.0+A 1 * A 1)$

$S=0: 5 * \times S 1 *(A 2+A L O G(A 1+A 2) / A 1)$

c

C

$7 \quad$ FTASQ $=(\times 0+A K * \times S I * \times S I) * * 2+(Y O-\times S I) * 2$

$A 1=S T H+2.0 * A K * X S I * C T H$

$A 2=C T H-2.0 * A K * X S I * S T H$

RBETA $=A T A N 2(A 1, A 2)$

$W=B(3) * B O+B(4) * S$

IF(W.LE.0.0) GOTO 8

$A l=0.6931471806 * E$ TASQ/ $(W * W)$

IFIAL.GE.25.0) GOTO 8

$A 1=\operatorname{EXP}(-A))$

GOTO 9

$8 \quad \triangle 1=0: 0$

$9 \quad I F(S-B(2) * B 0) 10,10,11$

$10 \quad R=B(1) * A)$

RETURN

$11 \quad R=B(1) * A 1 * S Q R T(B(2) * B O / S)$

RETURN

$1 \overline{2} \quad R=0.0$

RBETA $=$ THETA

RETURN

END

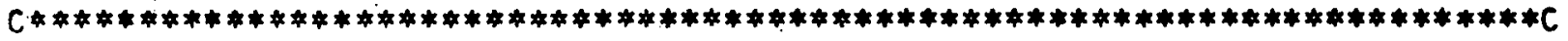

C

FUNCTION CUBRT $(X)$

C

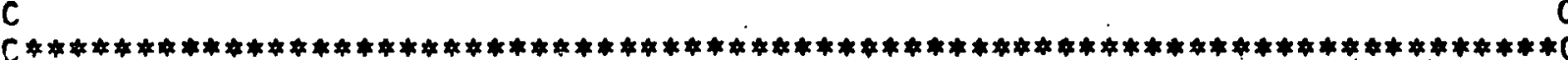

C

C RDUTINE CALCULATES THE CUBE-ROOT OF A REAL NUMBER.

C

$Y=X$

$S I G N=1.0$

I $F(Y) 1,3,2$

$1 \quad S I G N=-1.0$

$Y=-Y$

$2 \quad Y=0.333333333 * A L O G(Y)$

CUBRT $=S I G N$ \#EXP(Y) 


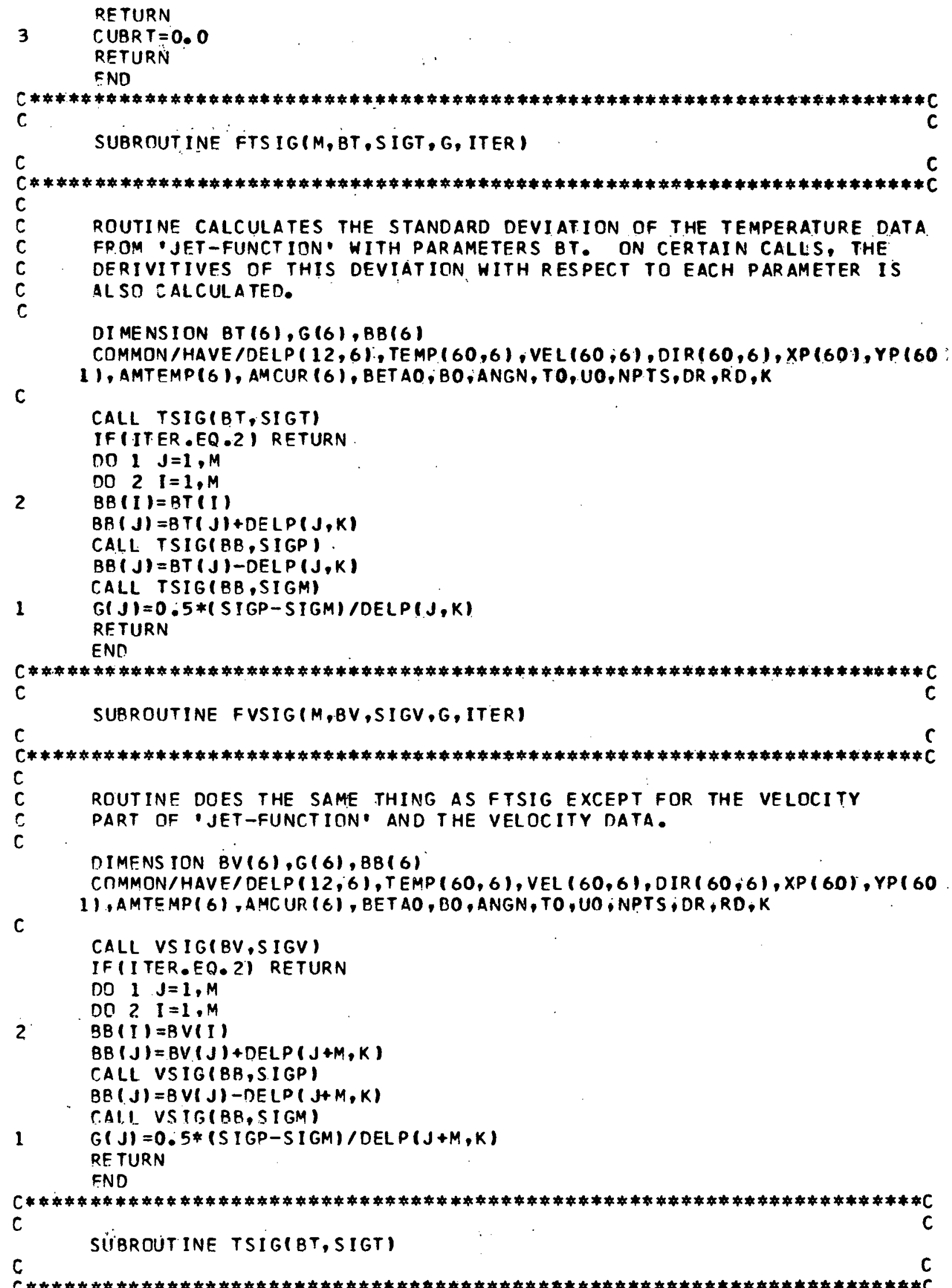


$c$

C ROUTINE CALCULATES THE STANDARD DEVIATION OF THE TEMPERATURE

C DATA FROM 'JET-FUNCTION' OF PARAMETERS BT.

C

DI MENSION BT (6)

COMMON /HAVE /OELP $(12,6)$, TEMP $(60,6) ; V E L(60,6), 0 I R(60 ; 6), X P(60) ; Y P(60$.

C

1), AMT EMP (6), AMCUR (6), BETAO,BO,ANGN,TO,UO,NPTS,DR,RD,K

$$
S I G T=0.0
$$

ANUM $=0.0$

DO $2 I=1$, NPTS

IF (TEMP $(I, K)) 2,1,1$

1 CALL TEMVEL (XP(I),YP(I),BT,BO,BETAO,RT,RBETA)

$S I G T=S I G T+1 T E M P(I, K)-R T * T O+R T * A M T E M P(K)-A M T E M P(K)) * * 2$

$A N U M=A N U M+1,0$

2

CONT INUF

DE GFR =A NUM-6.0

IF (DECFR.LT.0.5) DECFR=1.0

SI GT -SORT (SIGT/DEGFR)

RE TURN

FND

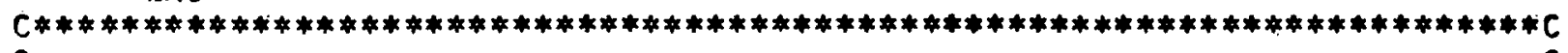

C

SUBROUTINE VSIG(BV,SIGVI

C

C

C

C ROUTINE DOES THE SAME THING AS TSIG ONLY FOR VELOCITY.

C

DIMENSIDN BV(6)

COMMON/HAVE/ DELP $(12,6)$, TEMP $(60 ; 6), V E L(60,6), D I R(60,6)$, XP $(60)$, YP(60

C

1), AMTEMP(6), AMCUR (6), BET AO,BO, ANGN,TO,UO,NPTS, DR,RD,K

$$
\begin{aligned}
& \text { SIGV }=0: 0 \\
& \text { ANUM=0.0 } \\
& \text { DO } 2 I=1, N P T S \\
& \text { IF }(V E L(I, K) 12,1,1
\end{aligned}
$$

1 CALL TEMVELIXP(I),YP(I),BV,BO,BETAO,RV,RBETA)

DEVBET $=(A N G N-O I R(I, K) \mid \star D R-R B E T A$

$S I G V=S I G V+(V E L(I, K) * C O S(D E V B E T)-R V * U 0 \div A M C U R(K) * C O S(R B E T A)) * 2$

$A N U M=A N U M+1.0$

2 CONT INUE

DEGFR $=A$ NUM-6.0

IF IDEGFP.LT.0.5) OOEGFR=1.0

$S$ I GV =SQRT (SI GV/DEGFR )

RETURN

END

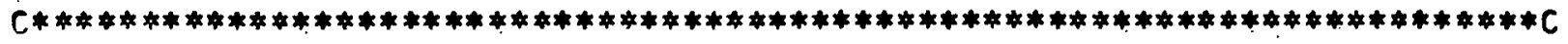

C

FUNCTION TAREA(TE,P)

C

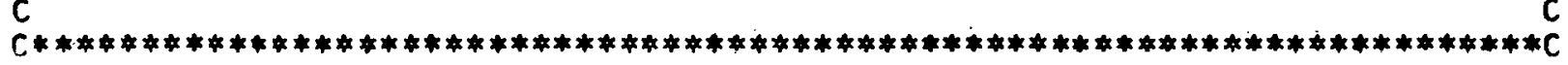

C

C FUNCTION CALCULATES THE APPROXIMATE ISOTHERM AREAS OF 'JET-

C FUNCTION' NEGLECTING. THE CURVATURE. OF. THE CENTERLINE.

C

DIMENSION P(12,6)

COMMON/HAVE IDELP $(12,6)$, TEMP $(60,6) ; V E L(60,6), D I R(60,6), X P(60), Y P(60$

C

1), AMTEMP( 6$)$, AMCUR (6), BETAO,BO,ANGN, TO,UO,NPTS, DR, RD, K

TAREA $=0.0$

$R=P(1, K) \neq(T O-A M T E M P(K)) / T E$ 


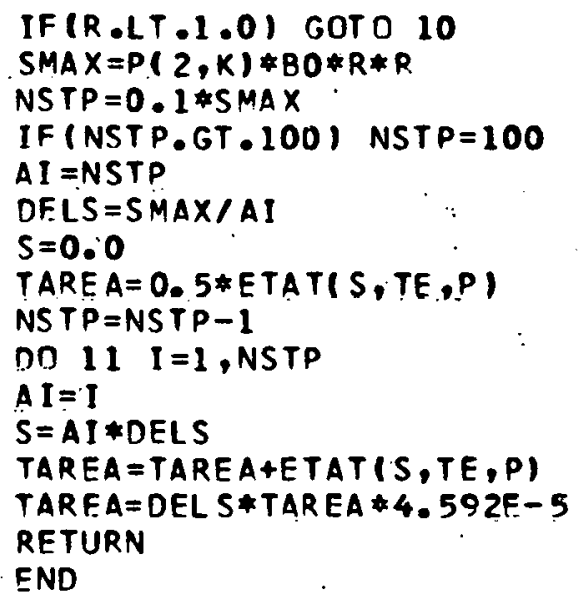

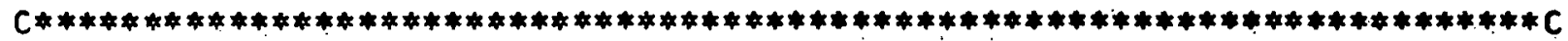
C

C

FUNCTION ETATIS, TE, P)

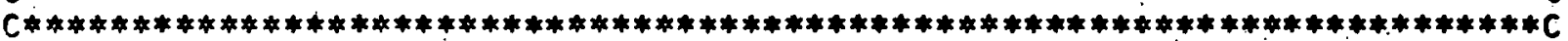

C

C FUNCT ION CALCULATES THE LATERAL DISTANCE OUT TO THE TE TEMPERATURE
C EXCESS ISOTHFRM.

C

C

DIMENSION $P(12,6)$

COMMON/HAVE/ DELP $(12,6)$, TEMP $(60,6), V E L(60,6), 01 R(60,6), X P(60), Y P(60$

1), AMTEMP( 6), AMCUR (6), BETAO,BO;ANGN,TO,UO,NPTS , DR,RD,K

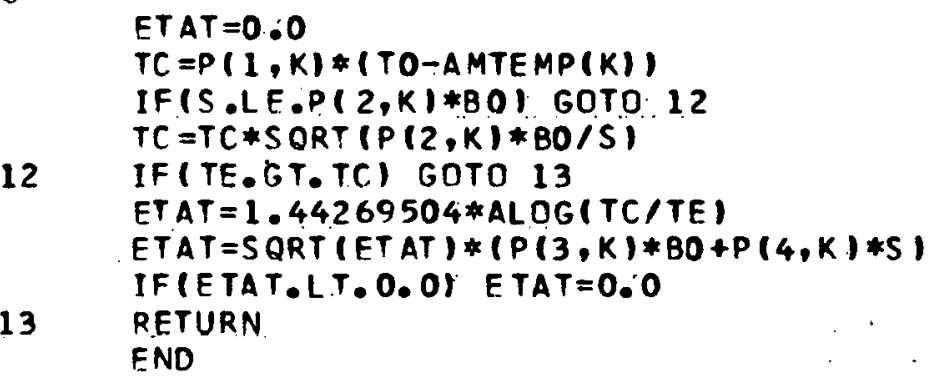

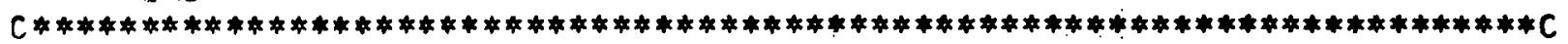

C

FUNCTION ETAVIS,VE,P\}

C

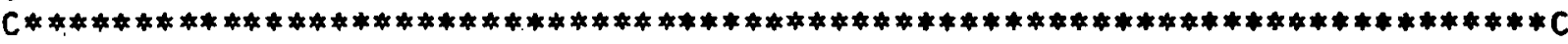

$\mathrm{C}$

C function CalCulates the lateral distance out to the Ve Velocity

C EXCESS POINT.

C DIMENSION P(12,6)

COMMON/HAVE/DELP $(12,6)$, TFMP $(60,6), V E(160,6), D I R(60,6), X P(60), Y P(60)$

C

1). AMTE MP(6), AMCUR (6), BET AO, BO, ANGN, TO ,UO,NPTS, DR,RD,K

ETAV $=0: 0$

$V C=P(7, K) * U 0$

$I F(S, L E \bullet P(8, K) * B O)$ GOTO 10

$V C=V C * S Q R T(P(8, K) * 80 / S)$

10 IF (VE:GT:VC) GOTO 11

ETAV $=1.44269504 * A L O G(V C / V E)$

ET AV $=S Q R T(E T A V) *(P(9, K) * B O+P(10, K) * S)$

11 RETURN

END 


\section{APPENDIX D \\ Fitting Parameters and Results}

This Appendix contains the final values of the sets of 12 parameters (six parameters for the fit to the temperature measurements and six for the fit to the velocity data) obtained from the fitting procedure described in Sec. V. The results for the five jet studies analyzed in this manner are included. Values are tabulated (Tables D.1-D.5) for each depth at which sufficient data were available to make the fitting procedure meaningful. Also given are outfall and ambient temperatures and velocities used. In addition, the differences between the measured values at each station and the values calculated from the final fitted functions are tabulated. The quantity $\Delta \mathrm{T}$ is defined as the calculated temperature excess minus the measured temperature excess at each data location; the quantity $\Delta u$ is defined as the difference between the calculated jet centerline component of the local jet velocity for each data point and the component of the measured velocity parallel to the fitted velocity centerline. Also included are the root-mean-square deviations $\sigma_{\mathrm{T}}$ and $\sigma_{\mathrm{u}}$ defined in Sec. $\mathrm{V}$ and $\overline{\Delta T}$ and $\overline{\Delta u}$, which are the average of the absolute values of the tabulated deviations. The quantities $\overline{\Delta T}_{\text {all }}$ and $\overline{\Delta u}_{\text {all }}$ are the average deviations for all depths.

TABLE D.1. Results of the Fitting Procedure for May 18, 1972, Data

$$
\begin{aligned}
& \mathrm{T}_{0}=17.7^{\circ} \mathrm{C} \\
& \mathrm{U}_{\mathrm{O}}=54.7 \mathrm{~cm} / \mathrm{scc} \\
& \mathrm{U}_{\mathrm{A}}=0.0 \mathrm{~cm} / \mathrm{sec}
\end{aligned}
$$

\begin{tabular}{c|ccccccc|}
\multicolumn{2}{c}{} & $0.5 \mathrm{~m}$ & $1.0 \mathrm{~m}$ & $1.5 \mathrm{~m}$ & $2.0 \mathrm{~m}$ & $2.5 \mathrm{~m}$ & $3.0 \mathrm{~m}$ \\
\hline $\mathrm{T}_{\mathrm{A}}\left({ }^{\circ} \mathrm{C}\right)$ & & 9.2 & 8.5 & 8.3 & 7.7 & 7.6 & 7.5 \\
$\theta_{\mathrm{n}}\left(\mathrm{C}^{\circ}\right)$ & & 8.5 & 9.2 & 9.4 & 10.0 & 10.6 & 10.2 \\
& & & & & & \\
& $\mathrm{~A}$ & 0.84 & 0.71 & 0.78 & 0.68 & 0.80 & \\
& $\alpha$ & 3.8 & 4.8 & 3.9 & 3.0 & 3.3 & \\
$\mathrm{C}$ & 0.8 & 1.3 & 0.4 & 2.0 & 0.2 & \\
$\mathrm{Y}$ & 0.39 & 0.35 & 0.41 & 0.13 & 0.19 & \\
$\mathrm{R}_{\mathrm{T}}$ & 0.77 & 0.75 & 0.81 & 0.72 & 0.61 & \\
$\mathrm{~K}_{\mathrm{T}}$ & 1.41 & 1.29 & 0.85 & 2.85 & 3.27 & \\
& & & & & & \\
$\mathrm{~B}$ & 1.47 & 1.32 & 1.28 & 1.24 & 0.83 & \\
$\mathrm{~B}$ & 4.6 & 3.7 & 3.4 & 3.0 & 1.4 & \\
$\mathrm{D}$ & 0.4 & 0.8 & 0.5 & 0.9 & 0.9 & \\
$\delta$ & 0.20 & 0.12 & 0.12 & 0.06 & 0.01 &. \\
$\mathrm{R}_{\mathrm{U}}$ & 1.04 & 0.95 & 1.02 & 1.03 & 0.85 & \\
$\mathrm{~K}_{\mathrm{u}}$ & 0.55 & 1.24 & 0.63 & 0.42 & 1.98 & \\
& & & & & &
\end{tabular}


TABLE D. 1 (Contd.)

\begin{tabular}{|c|c|c|c|c|c|c|}
\hline .. & $0.5 \mathrm{~m}$ & $1.0 \mathrm{~m}$ & $1.5 \mathrm{~m}$ & $2.0 \mathrm{~m}$ & $2.5 \mathrm{~m}$ & $3.0 \mathrm{~m}$ \\
\hline 1 & 0.0 & 0.5 & -- & -0.7 & --- & \\
\hline 2. & -0.6 & -0.6 & -0.4 & -0.5 & -0.2 & \\
\hline 3 . & $\cdots-$ & 1.0 & $\because$ & -0.9 & 0.2 & \\
\hline 4 & -0.7 & -0.4 & -0.2 & -0.7 & -0.4 & \\
\hline 5 & -1.8 & -1.0 & -0.7 & -0.8 & 0.0 & \\
\hline 6 & 0.4 & 0.2 & 0.7 & 0.5 & -0.5 & \\
\hline 7 & -- & $\therefore$ & $\ldots$ & -- & -.. & \\
\hline 8 & 0.5 & 0.5 & 1.0 & $-\ldots$ & -.. & \\
\hline 9 & 0.4 & 0.5 & -0.3 & -0.8 & 0.2 & \\
\hline 10 & 0.9 & 0.0 & 0.0 & 1.1 & 0.1 & $\cdot$ \\
\hline 11 & 0.6 & 0.6 & 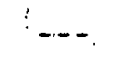 & 1.1 & -. & \\
\hline 12 & -1.4 & 0.1 & 0.0 & -.. & $\cdots$ & \\
\hline 13 & 0.7 & 0.9 & 1.7 & --- & -- & \\
\hline 14 & -1.5 & -2.0 & -1.9 & -1.4 & -- & \\
\hline 15 & 0.2 & 0.4 & 0.6 & 0.3 & $\because-$ & \\
\hline 16 & -0.2 & 0.0 & 0.4 & 0.5 & --- & \\
\hline 17 & 1.6 & 0.8 & 1.0 & 1.1 & 0.0 & \\
\hline 18 & -1.0 & -1.2 & -2.0 & -0.7 & -- & \\
\hline$\sigma_{\mathrm{T}}$ & 1.2 & 1.0 & 1.3 & 1.1 & $0 . .5$ & \\
\hline$\overline{\Delta T}$ & 0.8 & 0.6 & 0.8 & 0.8 & 0.2 & \\
\hline
\end{tabular}


TABLE D.1 (Contd.)

Station \#

$$
\Delta U=U_{\text {calc. }}-U_{\text {data }}(\mathrm{cm} / \mathrm{sec})
$$

\begin{tabular}{|c|c|c|c|c|c|c|}
\hline & $0.5 \mathrm{~m}$ & $1.0 \mathrm{~m}$ & $1.5 \mathrm{~m}$ & $2.0 \mathrm{~m}$ & $2.5 \mathrm{~m}$ & $3.0 \mathrm{~m}$ \\
\hline 1 & 5 & 3 & -- & 0 & -- & \\
\hline 2 & 0 & 0 & -2 & -3 & -5 & \\
\hline 3 & - & -2 & -- & 0 & 0 & \\
\hline 4 & 0 & 2 & 3 & 1 & 0 & \\
\hline 5 & 1 & 3 & -1 & -2 & -1 & \\
\hline 6 & -5 & -1 & 3 & 0 & 0 & \\
\hline 7 & -- & -- & -- & -- & -- & \\
\hline 8 & 1 & 1 & 0 & -- & $-\therefore$ & \\
\hline 9 & -2 & -4 & -7 & -2 & 1 & \\
\hline in & 1 & -1 & 4 & 10 & 0 & \\
\hline 11 & 6 & 3 & -- & 1 & -- & \\
\hline 12 & 0 & 0. & 0 & -. & -. & \\
\hline 13 & 2 & 3 & 4 & -- & -. & \\
\hline 14 & -6 & -4 & -5 & -8 & $\therefore$ & \\
\hline 15 & 9 & 9 & 9 & 3 & -- & \\
\hline 16 & -10 & -8 & -11 & -5 & $\therefore$ & \\
\hline 17 & -3 & 0 & -3 & 3 & -1 & \\
\hline 18 & -8 & 1 & 0 & 0 & - & \\
\hline$\sigma_{u}$ & 6 & 5 & 7 & 5 & 4 & \\
\hline$\overline{\Delta \mathbf{U}}$ & 4 & 3 & 4 & 3 & 1 & \\
\hline
\end{tabular}


TABLE D.2. Results of the Fitting Procedure for May 23, 1972, Data

$$
\begin{aligned}
\mathrm{T}_{\mathrm{O}} & =21.6{ }^{\circ} \mathrm{C} \\
\mathrm{U}_{\mathrm{O}} & =55.5 \mathrm{~cm} / \mathrm{sec} \\
\mathrm{U}_{\mathrm{A}} & =0.0 \mathrm{~cm} / \mathrm{sec}
\end{aligned}
$$

\begin{tabular}{|c|c|c|c|c|c|c|c|}
\hline & & $0.5 \mathrm{~m}$ & $1.0 \mathrm{~m}$ & $1.5 \mathrm{~m}$ & $2.0 \mathrm{~m}$ & $2.5 \mathrm{~m}$ & $3.0 \mathrm{~m}$ \\
\hline $\mathrm{T}_{\mathrm{A}}\left({ }^{\circ} \mathrm{C}\right)$ & & 14.3 & 12.6 & 12.1 & 11.6 & 11.3 & 10.8 \\
\hline$\theta_{0}\left(C^{\circ}\right)$ & & 7.3 & 9.0 & 9.5 & 10.0 & 10.3 & 10.8 \\
\hline & A & 0.84 & 0.91 & 0.87 & 0.85 & 0.85 & 0.96 \\
\hline & $\alpha$ & 5.6 & 4.4 & 3.9 & 3.7 & 3.8 & 2.7 \\
\hline & $\mathrm{C}$ & 1.0 & 0.7 & 0.7 & 0.9 & 0.9 & 0.5 \\
\hline & $\lambda$ & 0.27 & 0.37 & 0.32 & 0.21 & 0.14 & 0.20 \\
\hline ! & $\mathrm{R}_{\mathrm{T}}$ & 1.28 & 1.26 & 1.33 & 1.22 & 1.28 & 1.58 \\
\hline & $\mathrm{K}_{\mathrm{T}}$ & -2.56 & -2.58 & -3.56 & -1.88 & -2.64 & -7.06 \\
\hline & B & 1.04 & 0.98 & 0.9 .2 & 0.93 & 0.80 & 0.93 \\
\hline . & $B$ & 4.8 & 4.7 & 5.1 & 3.6 & 2.6 & 3.7 \\
\hline & $\mathrm{D}$ & 0.8 & 0.9 & 0.8 & 0.8 & 0.9 & 0.6 \\
\hline & $\delta$ & 0.17 & 0.09 & 0.04 & 0.03 & 0.02 & 0.01 \\
\hline & $\mathrm{R}_{\mathrm{u}}$ & 1.29 & 1.24 & 1.25 & 1.28 & 1.38 & 1.49 \\
\hline & $\mathrm{K}_{\mathrm{u}}$ & -2.11 & -1.76 & -1.90 & -2.03 & -2.84 & -3.93 \\
\hline
\end{tabular}


TABLE D. 2 (Contd.)

Station \#

$$
\Delta \mathrm{T}=\mathrm{T}_{\text {calc. }}-\mathrm{T}_{\text {data }}\left(\mathrm{C}^{\circ}\right)
$$

\begin{tabular}{|c|c|c|c|c|c|c|}
\hline & $0.5 \mathrm{~m}$ & $1.0 \mathrm{~m}$ & $1.5 \mathrm{~m}$ & $2.0 \mathrm{~m}$ & $2.5 \mathrm{~m}$ & $3.0 \mathrm{~m}$ \\
\hline 1 & -0.6 & -2.2 & -2.6 & -- & -.. & -.- \\
\hline 2 & 0.8 & 0.8 & 1.3 & 1.9 & 1.6 & $-\therefore$ \\
\hline 3 & -0.3 & 0.0 & -0.5 & -0.8 & -0.5 & -0.6 \\
\hline 4 & -0.9 & -0.7 & -1.1 & -1.7 & -1.5 & 0.3 \\
\hline 5 & -1.4 & -1.8 & -2.3 & $=2.9$ & -2.9 & -0.8 \\
\hline 6 & -2.2 & -3.1 & -3.0 & -1.9 & -1.2 & 0.3 \\
\hline 7 & 1.4 & 2.8 & 3.4 & 3.1 & 3.0 & -- \\
\hline 8 & 0.4 & 0.2 & 0.6 & 2.3 & $\ldots$ & -- \\
\hline 9 & 1.5 & 1.8 & 1.0 & -- & $\therefore$ & --- \\
\hline 10 & -0.3 & 0.2 & 0.5 & 0.6 & 0.6 & --- \\
\hline 11 & 0.0 & 0.6 & 1.4 & 0.0 & 1.2 & $\ldots$ \\
\hline 12 & -0.1 & 0.2 & -0.8 & 0.1 & -1.2 & -. \\
\hline 13 & -0.4 & -1.3 & -1.7 & -2.1 & -2.6 & -.. \\
\hline 14 & -0.1 & 1.5 & 0.0 & 1.1 & --- & -- \\
\hline 15 . & 1.0 & -0.1 & 0.4 & -0.2 & -- & -- \\
\hline 16 & 0.2 & 0.2 & 0.1 & -0.3 & 0.7 & -- \\
\hline 17 & 0.1 & -0.2 & 0.8 & 0.0 & 0.8 & --- \\
\hline 18 & 0.0 & -0.6 & -1.0 & -2.1 & -- & :- \\
\hline 19 & 0.4 & 0.5 & 0.7 & -0.1 & -- & $\because$ \\
\hline 20 & 0.6 & -0.4 & -0.2 & -0.6 & -0.6 & -0.8 \\
\hline 21 & -0.3 & -1.2 & -1.4 & -0.9 & $-0,1$ & -0.3 \\
\hline 22 & 1.4 & 1.8 & 1.8 & 1.4 & 1.6 & 1.8 \\
\hline 23 & -1.2 & -0.7 . & -0.6 & -0.6 & 0.1 & -0.6 \\
\hline 24 & 0.0 & 0.0 & -1.4 & 0.0 & 0.0 & 0.0 \\
\hline 25 & -0.6 & -0.3 & -0.9 & -1.1 & -1.6 & -0.8 \\
\hline 26 & 0.2 & -1.1 & -1.5 & 0.0 & -1.1 & -1.2 \\
\hline 27 & -0.3 & -0.1 & 0.4 & 1.0 & 1.0 & 0.3 \\
\hline 28 & -0.6 & -0.5 & -0.3 & -0.2 & -0.8 & -0.7 \\
\hline 29 & 0.2 & 0.1 & 0.4 & 0.4 & 0.3 & 0.6 \\
\hline$\sigma_{\mathrm{T}}$ & 0.9 & 1.3 & 1.6 & 1.5 & 1.7 & 1.0 \\
\hline$\overline{\Delta \mathrm{T}}$ & 0.6 & 0.9 & 1.1 & 1.0 & 1.1 & 0.6 \\
\hline & & 0.9 & & & & \\
\hline
\end{tabular}


TABLE D.2 (Contd.)

\begin{tabular}{|c|c|c|c|c|c|c|}
\hline \multirow[t]{2}{*}{ Station \# } & \multicolumn{5}{|c|}{$\Delta \mathrm{U}=\mathrm{U}_{\mathrm{calc}}-\mathrm{U}_{\mathrm{data}}(\mathrm{cm} / \mathrm{sec})$} & \multirow{2}{*}{$3.0 \mathrm{~m}$} \\
\hline & $0.5 \mathrm{~m}$ & $1.0 \mathrm{~m}$ & $1.5 \mathrm{~m}$ & $2.0 \mathrm{~m}$ & $2.5 \mathrm{~m}$ & \\
\hline 1 & -2 & 0 & 0 &.- &.-- & -- \\
\hline 2 & 33 & 28 & 21 & 24 & 16 & $\therefore$ \\
\hline 3 & -4 & 0 & 2 & -2 & 5 & -1 \\
\hline 4. & -12 & -11 & -14 & -12 & 3 & 2 \\
\hline 5 & -28 & -21 & -25 & -31 & -30 & -6 \\
\hline 6 & -13 & -21 & -19 & -7 & -2 & 7 \\
\hline 7 & 13 & 13 & 11 & 13 & 18 &.-- \\
\hline 8 & 39 & 33 & 23 & 21. & -- & --- \\
\hline 9 & -2 & -2 & 12 & -- & -- & -- \\
\hline 10 & -11 & -16 & 2 & 6 & -13 & $\ldots$ \\
\hline 11 & -11 & 4 & 10 & -4 & -5 & -- \\
\hline 12 & -1 & 0 . & 1 & -1 & -4 &.- \\
\hline 13 & 1 & -2 & 2 & 3 & 1 & -- \\
\hline 14 & -6 & -5 & -6 & -6 & $\cdots$ & -- \\
\hline 15 & -7 & 0 & -12 & -1 & -- & -- \\
\hline 16 & 2 & 2 & -1 & 2 & 8 & -- \\
\hline 17 & -1 & -8 & -4 & 1 & 2 & -- \\
\hline 18 & 4 & 6 & 5 & 2 & -- & --- \\
\hline 19 & 1 & 1 & 0 & 2 & --- & $\therefore$ \\
\hline 20 & 6 & -2 & -5 & -10 & -10 & .6 \\
\hline 21 & 2 & 0 & 7 & 4 & 7 & 0 \\
\hline 22 & 0 & -5 & -5 & -1 & 3 & 0 \\
\hline 23 & -7 & 0 & 0 & 0 & 3 & 3 \\
\hline 24 & 0 & 0 & 0 & 0 & 0 & 0 \\
\hline 25 & -8 & -8 & -8 & 0 & 0 & 0 \\
\hline 26 & 5 & 2 & -3 & 0 & 4 & 0 \\
\hline 27 & 2 & 5 & 5 & 6 & 0 & 0 \\
\hline 28 & 0 & 0 & 0 & 0 & 0 & 0 \\
\hline 29 & 6 & -2 & 0 & 0 & 0 & 0 \\
\hline$\sigma_{\mathrm{U}}$ & 14 & 13 & 11 & 11 & 11 & 4 \\
\hline$\overline{\Delta \bar{U}}$ & 8 & 7 & 7 & 6 & 6 & 2 \\
\hline & ${\overline{\Delta \mathrm{U}_{\mathrm{a}}}}_{\mathrm{a}}$ & $\mathrm{cm}$ & & & & \\
\hline
\end{tabular}


TABLE D.3. Results of the Fitting Procedure for July 13, 1972, Data

$$
\begin{aligned}
& \mathrm{T}_{\mathrm{O}}=20.3^{\circ} \mathrm{C} \\
& \mathrm{U}_{\mathrm{O}}=54.7 \mathrm{~cm} / \mathrm{sec} \\
& \mathrm{U}_{\mathrm{A}}=5.7 \mathrm{~cm} / \mathrm{sec}
\end{aligned}
$$

\begin{tabular}{|c|c|c|c|c|c|c|c|}
\hline & & $0.5 \mathrm{~m}$ & $1.0 \mathrm{~m}$ & $1.5 \mathrm{~m}$ & $2.0 \mathrm{~m}$ & $2.5 \mathrm{~m}$ & $3.0 \mathrm{~m}$ \\
\hline $\mathrm{T}_{\mathrm{A}}\left({ }^{\circ} \mathrm{C}\right)$ & & 13.0 & 12.2 & 11.4 & 11.0 & 10.6 & 10.3 \\
\hline$\theta_{0}\left(C^{0}\right)$ & & 7.3 & 8.1 & 8.9 & 9.3 & 9.7 & 10.0 \\
\hline & $A$ & 0.92 & 0.89 & 0.70 & 0.89 & 0.81 & \\
\hline & $\alpha$ & 6.2 & 4.4 & 4.6 & $4: 3$ & 4.9 & \\
\hline & $\mathrm{C}$ & 0.3 & 0.5 & 4.1 & 0.7 & 0.8 & \\
\hline & $\mathrm{R}_{\mathrm{T}}$ & 0.97 & 0.97 & 0.88 & 0.94 & 0.98 & \\
\hline$\because \mathrm{K}$ & $K_{T}^{1}$ & -0.74 & -0.73 & 0.07 & 0.05 & -0.26 & \\
\hline & $\mathrm{B}$ & 1.26 & 1.22 & 1.05 & 1.26 & 0.95 & \\
\hline & $B$ & 7.4 & 5.3 & 4.8 & 7.5 & 4.3 & \\
\hline & $\mathrm{D}$ & 0.1 & 0.4 & 0.3 & 0.7 & 0.6 & \\
\hline & $\delta$ & 0.24 & 0.18 & 0.16 & 0.03 & -0.01 & \\
\hline & $\mathrm{R}_{\mathrm{u}}$ & 0.94 & 0.93 & 0.98 & 0.96 & 0.96 & \\
\hline & $\mathrm{K}_{\mathrm{u}}$ & -0.09 & -0.11 & -0.18 & -0.24 & -0.07 & \\
\hline
\end{tabular}


TABLE D. 3 (Contd.)

Station \#

$$
\Delta \mathrm{T}=\mathrm{T}_{\text {calc }}-\mathrm{T}_{\text {data }}\left(\mathrm{C}^{\circ}\right)
$$

\begin{tabular}{|c|c|c|c|c|c|c|}
\hline & $0.5 \mathrm{~m}$ & $1.0 \mathrm{~m}$ & $1.5 \mathrm{~m}$ & $2.0 \mathrm{~m}$ & $2.5 \mathrm{~m}$ & $3.0 \mathrm{~m}$ \\
\hline 1 & 0.1 & 0.3 & 1.3 & 0.8 & 0.0 & \\
\hline 2 & 0.3 & 0.0 & 0.6 & 0.0 & 1.4 & \\
\hline 3 & -0.6 & -0.6 & -0.9 & -0.3 & -0.6 & \\
\hline 4 & 0.4 & 0.6 & -1.2 & -0.1 &.- & \\
\hline 5 & 0.0 & -0.1 & 0.9 & $\therefore$ & $\cdots$ & \\
\hline 6 & -0.4 & -0.8 & -0.6 & -2.1 & -1.4 & \\
\hline 7 & 0.8 & 1.0 & 0.8 & 1.0 & -0.4 & \\
\hline 8 & -0.5 & -1.1 & -0.8 & -1.0 & -0.9 & \\
\hline 9 & $-0: 9$ & -1.1 & -1.3 & -0.5 & -1.6 & : \\
\hline 10 & 0.8 & 0.4 & 0.4 & -1.0 & -0.7 & \\
\hline 11 & -0.3 & -0.6 & -0.1 & $\ldots$ & -.- & \\
\hline 12 & -0.1 & 0.2 & $0: 2$ & 1.1 & -0.7 & \\
\hline 13 & 0.1 & 0.4 & 0.5 & 0.8 & 1.1 & \\
\hline 14 & 0.1 & 0.6 & 1.1 & 1.1 & 1.5 & . \\
\hline 15 & 0.4 & 0.7 & -0.2 & 0.0 & -0.7 & \\
\hline 16 & -0.2 & 0.3 & -0.8 & -0.6 & -0.4 & \\
\hline 17 & 0.1 & 1.1 & 0.2 & 0.8 & 0.2 & \\
\hline$\sigma_{\mathrm{T}}$ & 0.6 & 0.8 & $1 . \overline{0}$ & 1.2 & 1.3 & \\
\hline$\overline{\Delta \mathrm{T}}$ & 0.4 & 0.6 & 0.7 & $\ddot{0.8}$ & 0.8 & . \\
\hline
\end{tabular}


TABLE D. 3 (Contd.)

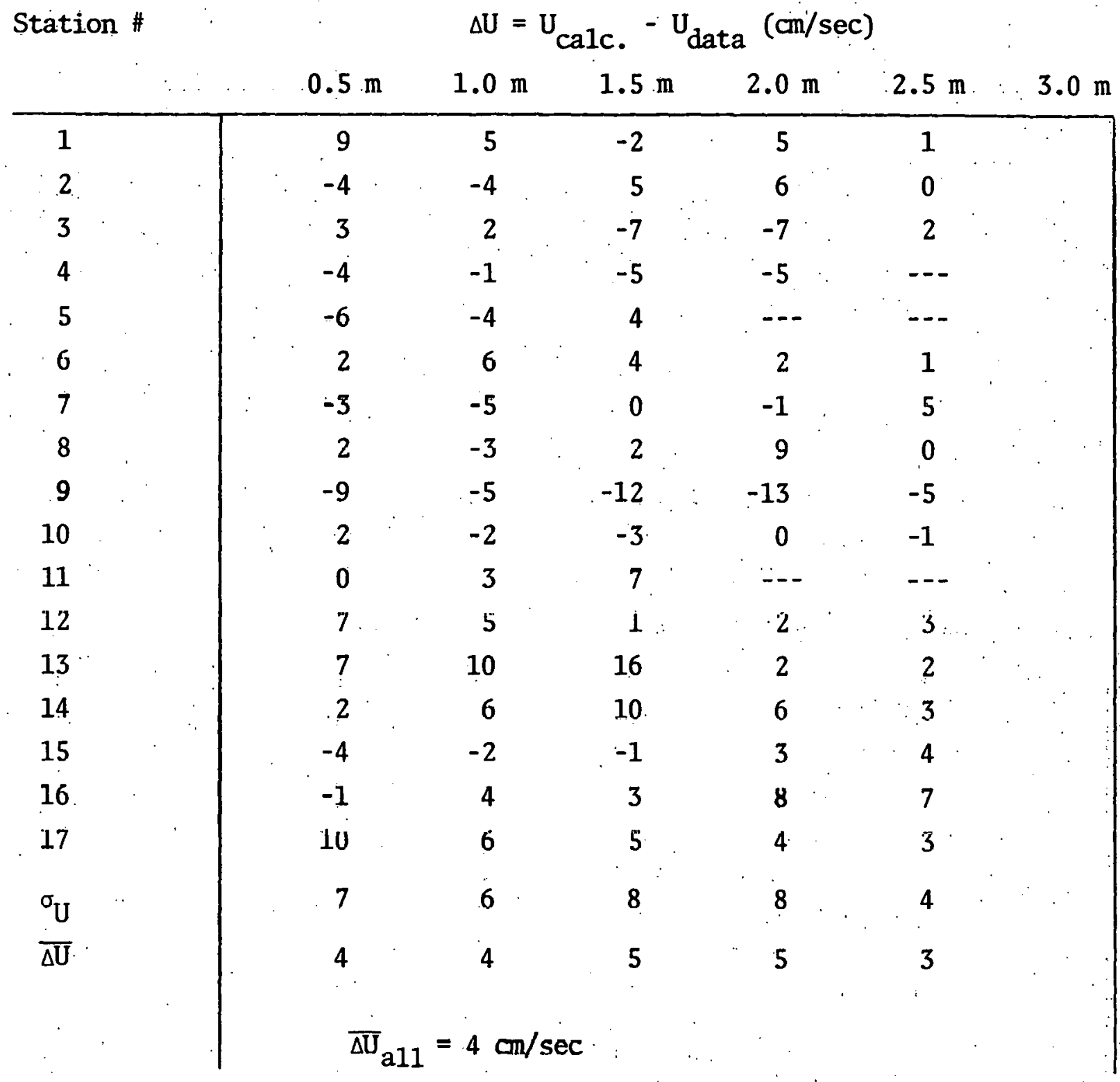


TABLE D.4. Results of the Fitting Procedure for September 9, 1972, Data

$$
\begin{aligned}
\mathrm{T}_{\mathrm{O}} & =24.5^{\circ} \mathrm{C} \\
\mathrm{U}_{\mathrm{O}} & =54.7 \mathrm{~cm} / \mathrm{sec} \\
\mathrm{U}_{\mathrm{A}} & =2.2 \mathrm{~cm} / \mathrm{sec}
\end{aligned}
$$

\begin{tabular}{|c|c|c|c|c|c|c|}
\hline & $0.5 \mathrm{~m}$ & $1.0 \mathrm{~m}$ & $1.5 \mathrm{~m}$ & $2.0 \mathrm{~m}$ & $2.5 \mathrm{~m}$ & $3.0 \mathrm{~m}$ \\
\hline $\mathrm{T}_{\mathrm{A}}\left({ }^{\circ} \mathrm{C}\right)$ & 16.3 & 15.2 & 14.4 & 14.1 & 13.9 & 13.8 \\
\hline$\theta_{0}\left(C^{\circ}\right)$ & 8.2 & 9.3 & 10.1 & 10.4 & 10.6 & 10.7 \\
\hline A & 0.79 & 0.68 & 0.66 & 0.88 & 0.31 & \\
\hline$\alpha$ & 4.5 & 3.7 & 2.1' & 2.0 & 0.4 & \\
\hline C & 0.1 & 1.1 & 2.1 & 2.0 & 0.8 & \\
\hline$\lambda$ & 0.64 & 0.58 & 0.45 & 0.26 & 0.61 & \\
\hline$\dot{\mathrm{R}} \mathrm{T}$ & 0.98 & 1.07 & 1.22 & 1.34 & 1.21 & \\
\hline $\mathrm{K}_{\mathrm{T}}$ & -0.44 & -1.25 & -2.18 & -3.08 & $-0: 45$ & \\
\hline B & 1.03 & 0.85 & 1.00 & 0.99 & & \\
\hline$B$ & 4.4 & 3.0 & 4.0 & 3.9 & & \\
\hline$D$ & 0.8 & 0.6 & 1.2 & 1.2 & & \\
\hline$\delta$ & 0.22 & 0.20 & -0.02 & -0.06 & : & \\
\hline $\mathrm{R}_{\mathrm{u}}$ & 0.98 & 0.98 & 0.97 & 1.01 & & \\
\hline $\mathrm{K}_{\mathrm{u}}$ & 0.58 & 1.24 & 0.82 & 0.60 & & \\
\hline
\end{tabular}


TABLE D. 4 (Contd.)

Station \#

$$
\Delta \mathrm{T}=\mathrm{T}_{\text {calc. }}-\mathrm{T}_{\text {data }}\left(\mathrm{C}^{\circ}\right)
$$

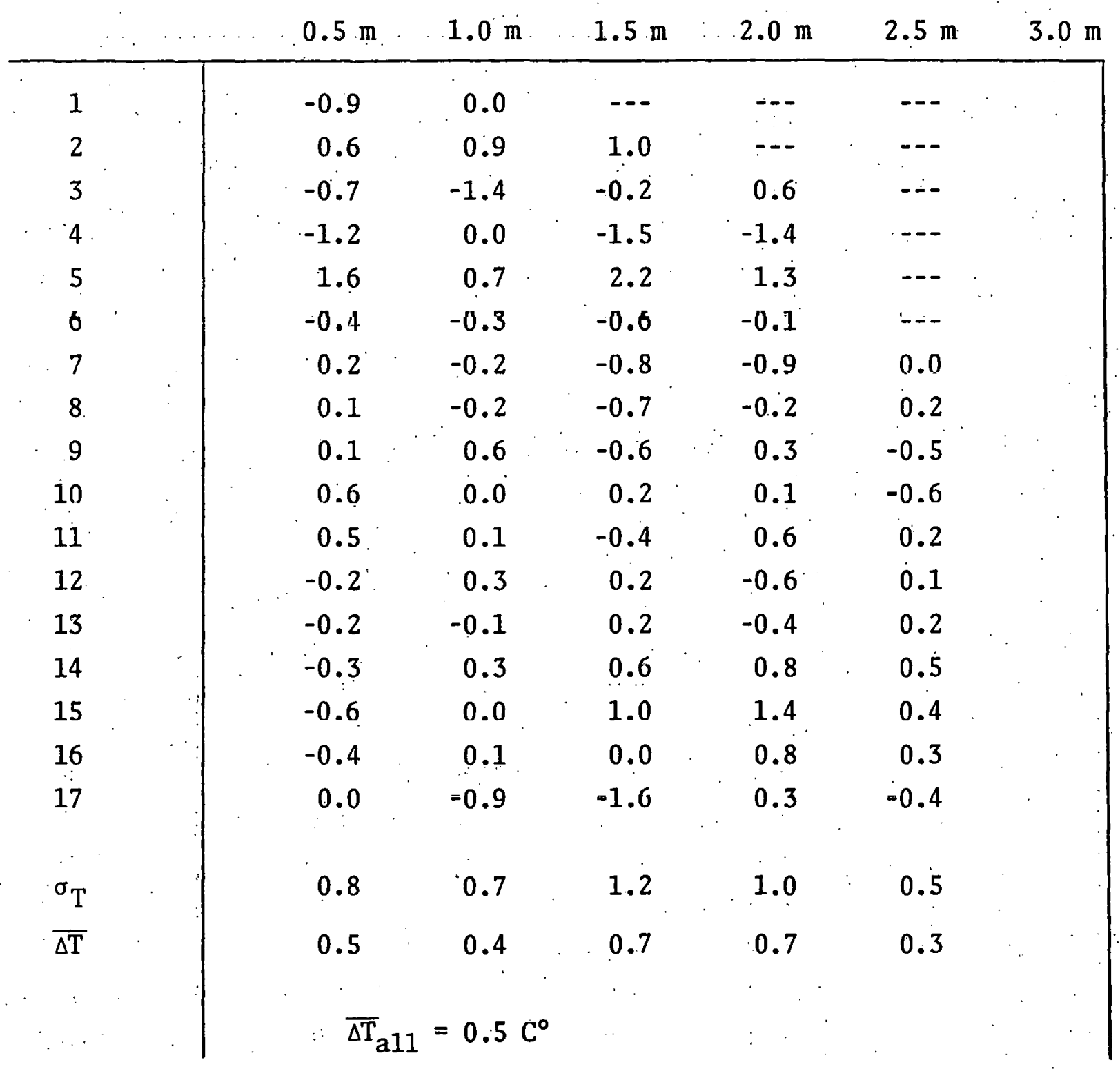


TABLE D. 4 (Contd.)

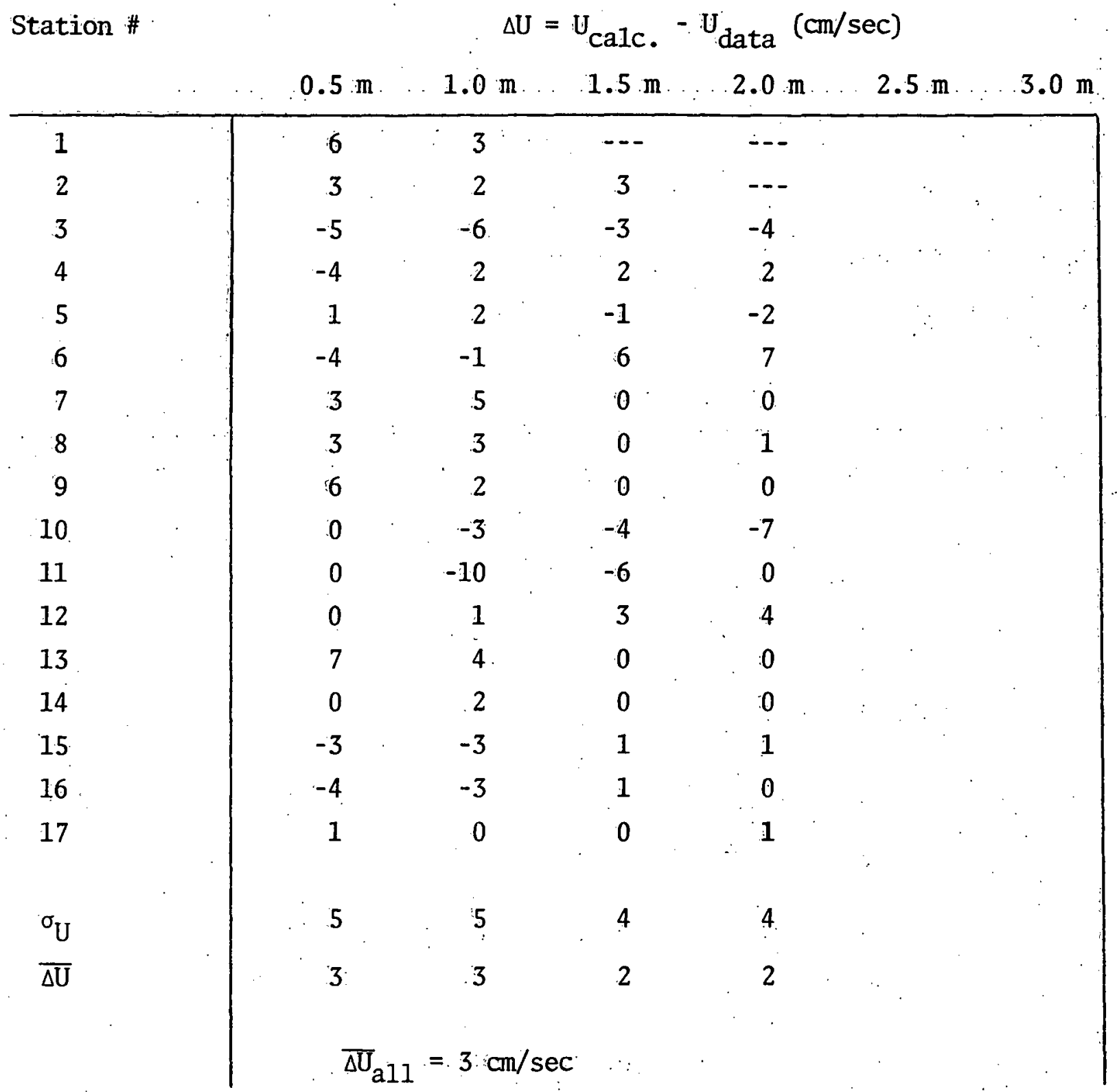


TABLE D.5. Results of the Fitting Procedure for October 10, 1972, Data

$$
\begin{aligned}
& \mathrm{T}_{\mathrm{O}}=22.2{ }^{\circ} \mathrm{C} \\
& \mathrm{U}_{\mathrm{O}}=42.3 \mathrm{~cm} / \mathrm{sec} \\
& \mathrm{U}_{\mathrm{A}}=0.0 \mathrm{~cm} / \mathrm{sec}
\end{aligned}
$$

\begin{tabular}{|c|c|c|c|c|c|c|c|}
\hline & & $0.5 \mathrm{mI}$ & $1.0 \mathrm{~m}$. & $1.5 \mathrm{~m}$ & $2.0 \mathrm{~m}$ & $2.5 \mathrm{~m}$ & $3.0 \mathrm{~m}$ \\
\hline $\mathrm{T}_{\mathrm{A}}\left({ }^{\circ} \mathrm{C}\right)$ & . & 13.9 & 13.0 & 12.7 & 12.8 & 12.7 & 12.7 \\
\hline$\theta_{0}\left(C^{0}\right)$ & & 9.2 & 9.2 & 9.5 & 9.6 & 9.5 & 9.5 \\
\hline & A & 0.85 & 0.59 & 0.48 & & & \\
\hline & $\alpha$ & 4.6 . & 1.4 & 0.8 & & & \\
\hline & $\mathrm{C}$ & 2.0 & 0.6 & 0.2 & & & \\
\hline & $\lambda$ & 0.52 & 0.85 & 0.46 & & & \\
\hline & $\mathrm{R}_{\mathrm{T}}$ & 1.02 & 1.13 & 1.18 & & & \\
\hline & $\mathrm{K} T$ & 0.29 & $=0.93$ & 0.61 & & & $\because$ \\
\hline & B & 0.52 & 0.58 & 0.22 & & & \\
\hline & $\beta$ & 1.9 & 0.8 & 0.2 & & & \\
\hline$\cdots$ & $\mathrm{D}$ & 0.2 & 0.2 & 0.7 & & & \\
\hline & $\delta$ & 0.29 & 0.19 & 0.00 & & & \\
\hline & $\mathrm{R}_{\mathrm{u}}$ & 1.27 & 1.12 & 1.16 & & & \\
\hline & $\mathrm{K}_{\mathrm{u}}$ & -8.29 & 3.14 & 1.28 & & & \\
\hline
\end{tabular}


TABLE .D.5 (Contd.)

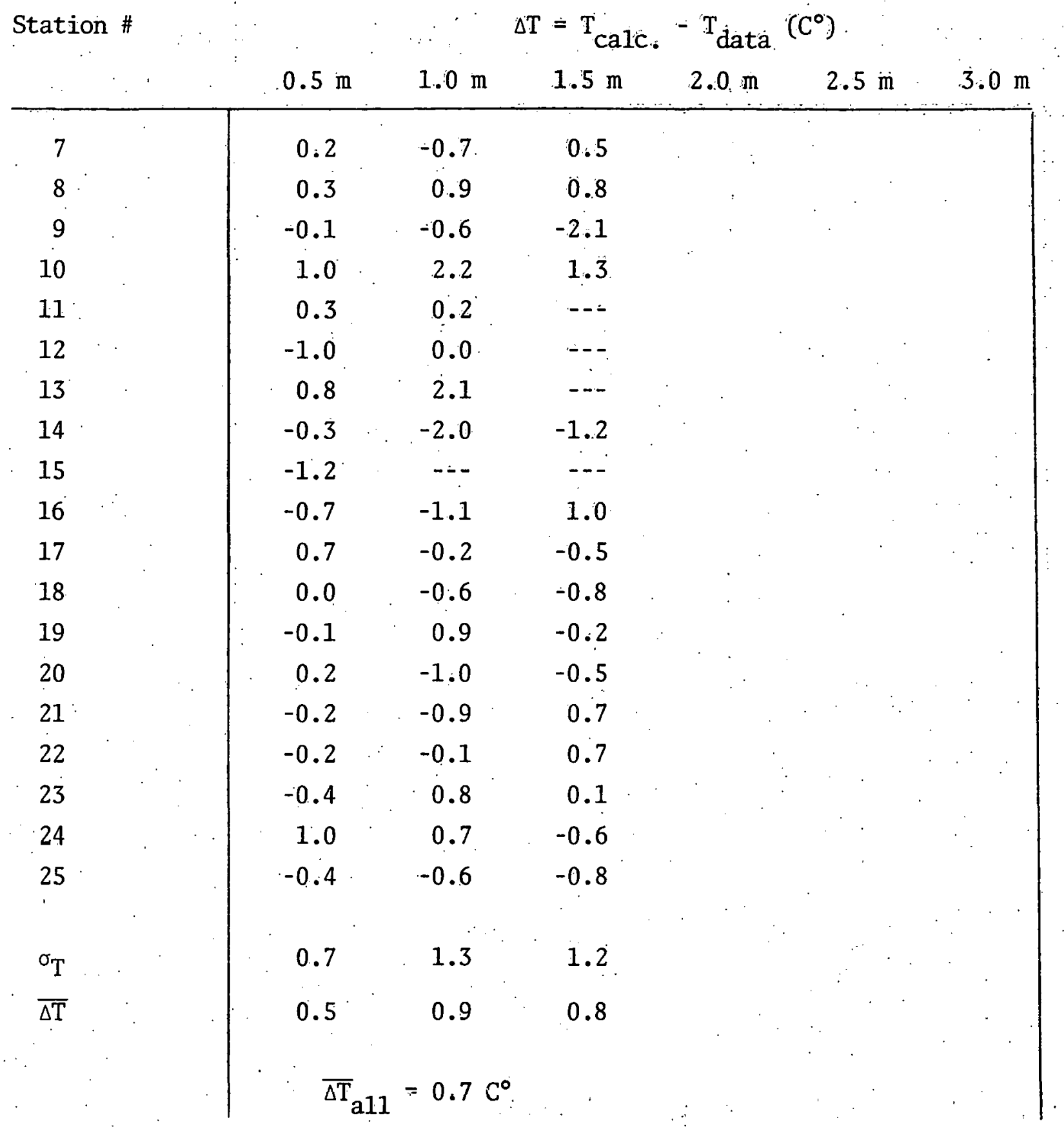


TABLE D. 5 (Contd.)

Station \#

$$
\Delta \mathrm{U}=\mathrm{U}_{\text {calc. }}-\mathrm{U}_{\mathrm{data}}(\mathrm{cm} / \mathrm{sec})
$$

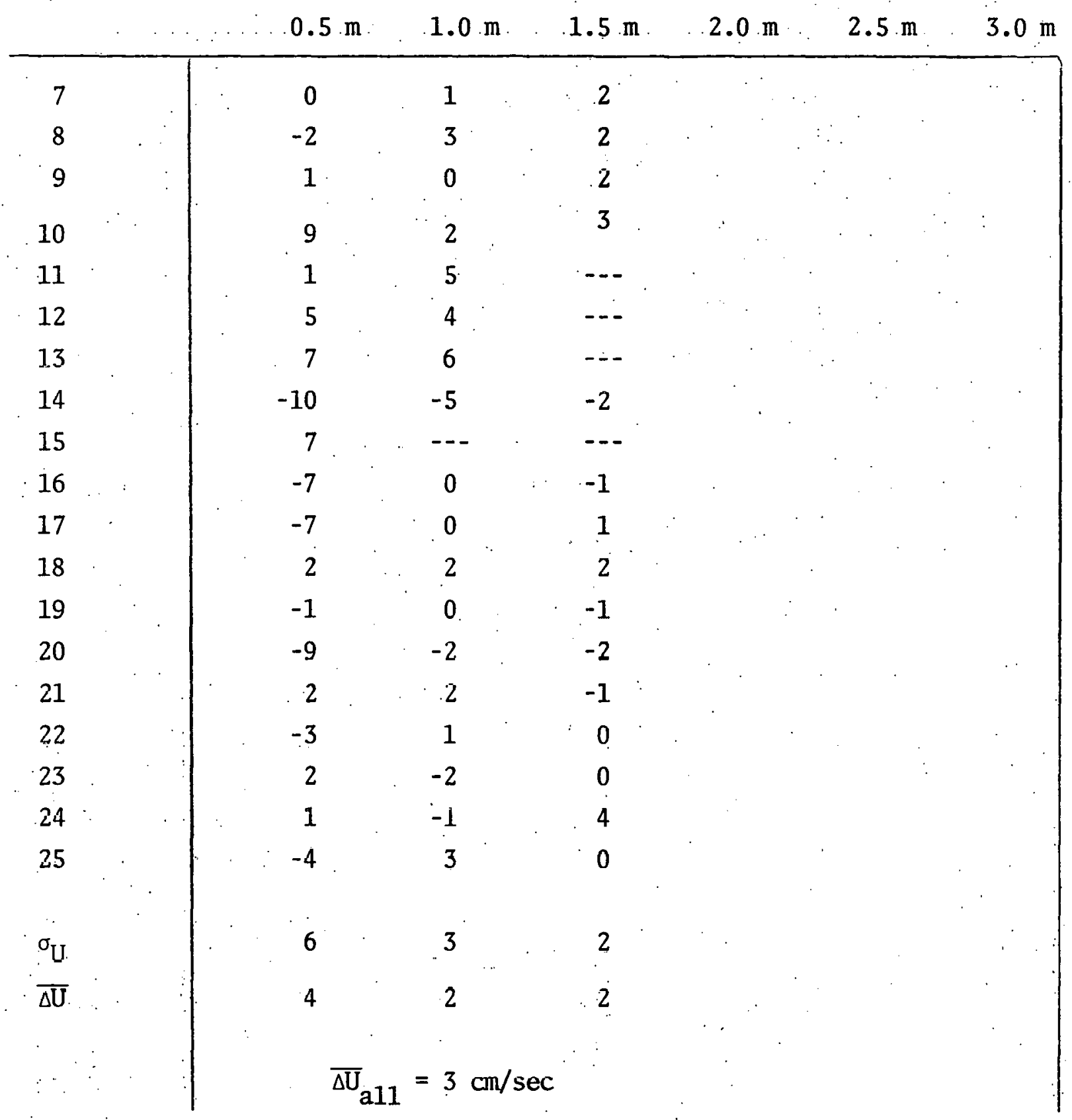




\section{ACKNOWLEDGMENTS}

In a study requiring extensive field work the cooperation and help of a number of people besides the principal authors is often required. In this regard we would like to acknowledge the help of Messrs. Conrad Tome, Paul Siebold, and Richard Zeren. We would particularly like to acknowledge Mr. Barton Hoglund for his general help and assistance in the design and implementation of the field program.

We are grateful for the critical comments and suggestions made by Mr. William Dunn involving the JETFIT fitting procedure and model-data comparisons, and we appreciate the programming assistance of Mr. Stanley Zawadzki in reference to the analytical models evaluated in this study.

We are also grateful to Messrs. Walter Clapper and Robert Neisius. for their graphic art contributions, and we would particularly like to acknowledge Ms. Maria Pacholok's typing and retyping several drafts of this report. 


\section{REFERENCES}

1. J. A. Hoopes, R. W. Zeller, and G. A. Rohlich, Heat Dissipation and Induced Circulations From Condenser Cooling Water Discharges Into Lake Monona, Report No. 35, Department of Civil Engineering, The University of Wisconsin (Feb 1968).

2a. T. Hayashi and N. Shuto, "Diffusion of Warm Water Jets Discharged Horizontally at the Water Surface," Proceedings of the International Association for Hydraulic Research, Colorado State University, Fort Collins, Colorado, Vol. 4, pp. 47-59, September 1967.

2b. T. Hayashi and N. Shuto, "Diffusion of Warm Cooling Water Discharged from a Power Plant," Proceedings of the 11th Conference on Coastal Engineering, Institution of Civil Engineers, Session AVI, pp. 13-16, September 16-20, 1.968 .

3. A. Wada, "Numerical Analycio of Distributiun of Flow and l'hermal Diffusion Caused by Outfall of Cooling Water," Proceedings of the Thirteenlh Corlgress of the Internationat Association fur Hydraulic Research, pp. 335-342, August 31-September 5, 1969.

4. H. H. Carter, A Preliminary Report on the Characteristics of a Heated Jet Discharged Horizontally irto a l'ransverse current, Fart 1-Constartt Depth, Tech. Report No. 61, Chesapeake Bay Institute, The Johns Hopkins University, Baltimore, Md. (Nov 1969).

5. L. H. Motz and B. A. Benedict, Heated Surface Jet Discharged Into $A$ Flowing Ambient Stream, Department of Environmental and Water. Resources Engineering, Vanderbilt University, Nashville, Tennessee, Report No. 4 (Aug 1970).

6a, 7. R. C. Y. Koh and L. Fan, Mathematical Models for the Prediction of Temperature Distributions Resulting from the Discharge of Hoated Water Into Large Bodies of Water, National Environmental Protection Agency Report, Water Pollution Control Research Series 16130 DWO 10/70 (Oct 1970).

6b. R. C. Y. Koh, Two-Dimensional Surface Warm Jets, ASCE, J. Hydraul. Div., 819-836 (June 1971).

8a. R. E. Barry and D, P. Hoffman, "Computar Model for a Thermal Plune," Mechanical Division, The Detroit Edison Company, Detroit, Mich., paper presented at ASCF National Resources Engineering Meeting, Phoenix, Arizona, January 11-15, 1971.

8b. D. P. Hoffman, "lhermal Plume Mode1," Mechanical Division, The Detruit Edison Company, Detrult, Mich., paper presented at Fifteenth Conference on Great Lakes Research, Madison, Wis., April 5-7, 1972.

9. K. Stolzenbach and D. R. F. Harleman, An Analytical and Experimental Investigation of Surface Discharges of Heated Water, Ralph M. Parsons Laboratory for Water Resources and Hydrodynainics, Massachusetts Institute of Technology, Cambridge, Report No. 135 (Feb 197i).

10a. R. W. McLay, M. S. Hundal, F. Martinek, and E. B. Henson, Mathematical Modeling of Nuclear Plant Thermal Effluents in Lake. Champlain, ASME, Winter Annual Meeting, Paper No. 71-WA/Pwr-4, November 26-December 2 , 1971. 
10b. M. S. Hundal, F. Martinek, E. B. Henson, and R. W. McLay, "A Computer Model for Predicting Thermal Discharges in Large Lakes," Department of Mechanical Engineering, University of Vermont, Burlington, paper presented at Fifteenth Conference on Great Lakes Research, Madison, Wis., April 5-7, 1972.

10c. R. W. McLay, M. S. Hundal, F. Martinek, and E. B. Henson, A Mathematical Aralysis of Thermal Polzution of Lakes and Estuaries, Department of Mechanical Engineering, University of Vermont, Burlington (May 1972).

11a. H. Stefan, Surface Discharge of Heated Water, Part I: Three Dimensional Jet-Type Surface Plumes in Theory and in the Laboratory, Department of Civil and Mineral Engineering, St. Anthony Falls Hydraulic Laboratory, Project Report No. 126, University of Minnesota, Minneapolis (1971).

11b. H. Stefan, "Analytical Jet-Type Model of Heated Water Surface Plumes," Department of Civil and Mineral Engineering, St. Anthony Falls Hydraulic Laboratory, University of Minnesota, Minneapolis, Minnesota, paper presented at Fifteenth Conference on Great Lakes Research, Madison, Wis., April 5-7, 1972.

12. E. Prych, A Warm Water Effluent Analyzed as a Buoyant Surface Jet, Sveriges Meteorologiska Och Hydrologiska Institut, Serie Hydrologi. Nr. 21, Stockholm, 1972 .

13. J. F. Paul and W. J. Lick, "A Numerical Model for a Three-Dimensional Variable-Density Jet,". School of Engineering and Department of Geology, Case Western Reserve University, Cleveland, Ohio, paper presented at the 16th Conference on Great Lakes Research, Huron, Ohio, April 16-18, 1973.

14. F. Engelund and F. Pedersen, Surface Jet at SmaZl Richardson Numbers, J. Hydraul. Div., Proc. Am. Soc. Civil Eng. 99(HY3), Proc. Paper 9588, pp. 405-416 (Mar 1973).

15a. W. Waldrop and R. Farmer, Three-Dimensional Computation of Buoyant Plumes, Coastal Studies Institute and Department of Chemical Engineering, Louisiana State University, Baton Rouge (Oct 1973).

15b. R. Farmer and P. Bryant, A Mathematical Model for the Los Angelesliong Beach Harhor, research proposal submitted to the Environmental Protection Agency by the Chemical Engineering Department, Louisiana State University, Baton Rouge (Oct 1972).

16. A. Wada, Numerical Analysis of Distribution of Flow and Thermal Diffusion Caused by Outfall of Cooling Water, Coastal Eng. In Japan 11 (1968).

17. D. Pritchard, "Design and Siting Criteria for Once-Through Cooling Systems," presented at American Institute of Chemical Engineers, 68th Annual Meeting, Houston, Texas, March 2, 1.971.

18. T. R. Sundaram, C. C. Easterbrook, K. R. Piech, and G. Rudinger, An Investigation of the Physical Effects of Thermal Discharges Into Cayuga Lake (Analytical Study), CAL No. VT - 2616-0-2, Cornell Aeronautical Laboratory, Inc. (Nov 1969).

19a. J. B. Bryce, W. R, Etter, and E. H. Dye, Water Environmental Studies and Water Quality Control at Ontario Hydro, The Hydro-Electric Power Commission of Ontario, Generation Projects Division, Toronto, Canada (June 16, 1971). 
19b. R. V. Elliott and D. G. Harkness, "A Phenomenological Model for the Prediction of Thermal Plumes in Large Lakes," The Hydro-Electric Power Commission of Ontario, Toronto, Canada; paper presented at Fifteenth Conference on Great Lakes Research, Madison, Wisconsin, April 5-7, 1972.

20. W. Giles, N. Johnson, G. McComb, F. Morris, J. Sche11, and J. Young, A Thermal Effluent Analysis for Electric Power Generating Plants, Reentry and Environmental Systems Division, General Electric, Technical Information Series No. 71SD257 (Sept 20, 1971).

21. L. A. Loziuk, J. C. Anderson, and T. Belytschko, Hydrothermal Analysis by the Finite Element Method, ASCE, J. Hydraul. Div. 98, No. HY11 (Nov 1972).

22. D. Brady and J. Geyer, Development of a General Computer Model for Simulating Thermal Discharges in Three Dimensions, Report No. 7, Department of Geography and Environmental Engineering, The Johns Hopkins University, Baltlinore, Md. (Feb 1972).

23. H. Till, A Computer Model for Three-Dimerisioral Simulation of Thermal. Discharges into Rivers, doctoral dissertation, Department of Nuclear Engineering, University of Missouri, Rolla (1973).

24. D. W. Pritchard, "Fate of and Effect of Excess Heat D1scharged Into Lake Michigan with Specific Application to the Condenser Cooling Water Discharge from the Zion Nuclear Power Station," testimony at AEC Licensing Hearings for Zion Operating Permit, Chicago, Ill. (June 1973).

25. H. H. Carter, E. W. Schiemer, and R. Regier, The Buoyant Surface Jet Discharging Normal to An Ambient Flow of Various Depths, Technical Report No. 81, Chesapeake Bay Institute, The Johns Hopkins University, Baitimore, Md. (Feb 1973).

26. B. R. Morton, On a Momentum-Flux Diagram for Turbulent Jets, Plumes, and Wakes, J. Fluid Mech. 10 (1961).

27. L. Fan, Trinbulont Buoyant Jets into Stratified on Flowing Ambient Fluids, Tech. Report No. KH-R-15, W. M. Keck Laboratory of Hydraulics and Water Resources, California Institute of Technology, Pasadena (June 1967).

28. G. N. Abramovich, The Theory of Turbulent Jets, M.I.T. Press, Massachusetts Institute of Technology, Cambridge (1963).

29. 'I'. Ellison and J. Turner, Turbulent Entrainment in Stratified Flows, J. Fluid Mech. 6, 423 (1959).

30. F. L. Scarpace and T. Green III, Dynamic Surface Temperature of Thermal. Plumes, Water Resources Research $9(1)$ (Feb 1973). 